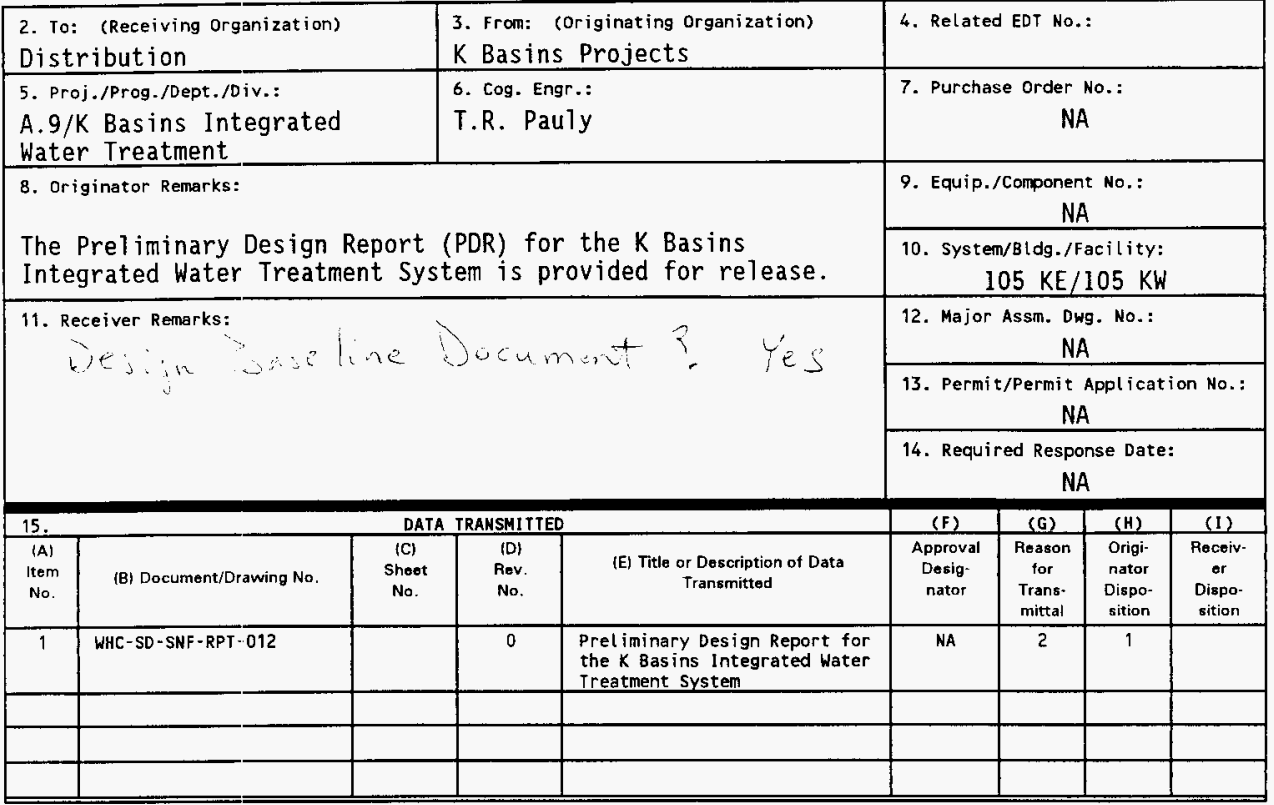

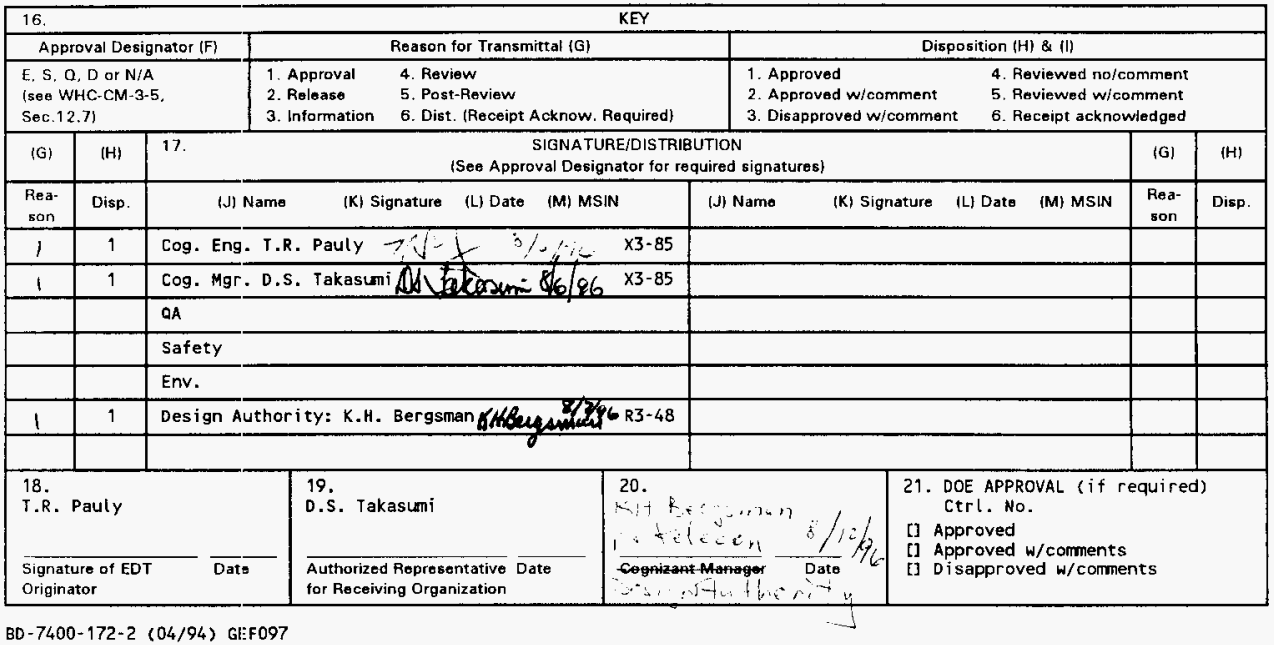

BD-7400-172-2 (04/94) GE:F097 


\title{
PRELIMINARY DESIGN REPORT for the K BASINS INTEGRATED WATER TREATMENT SYSTEM
}

\author{
D.E. Ball/M.A. Knight/T.R. Pauly
}

Westinghouse Hanford Company, Richland, WA 99352

U.S. Department of Energy Contract DE-AC06-87RL10930

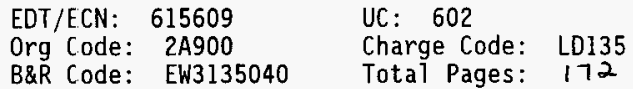

Key Words: K Basins, Integrated Water Treatment, PDR

Abstract:

This Preliminary Design Report (PDR) provides a revised concept for the $K$ Basins Integrated Water Treatment Systems (IWTS). This PDR

incorporates the 11 recommendations made in a May 1996 Value Engineering session into the Conceptual Design, and provides new flow diagrams, hazard category assessment, cost estimate, and schedule for the IWTS Subproject.

TRADEMARK DISCLAIMER. Reference herein to any specific comercial product, process, or service by trade rame, trademark, manufacturer, or otherwise, does not necessarily constitute or imply its endorstment, recommendation, or favoring by the United States Government or any agency thereof or its coritractors or subcontractors.

Printed in the United States of America. To obtain copies of this document, contact: WHC/BCS Documerit Control Services, P.0. Box 1970, Mailstop H6-08, Richland WA 99352, Phone (509) 372-2420; Fax (509) 376-4989.
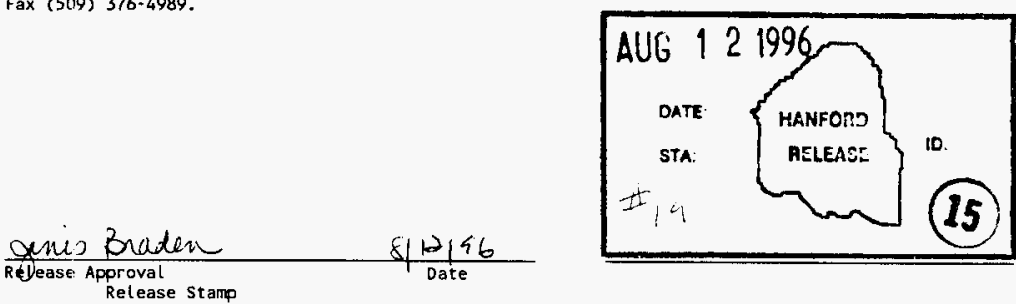

\section{Approved for Public Release}




\title{
PRELIMINARY DESIGN REPORT
}

\author{
FOR THE \\ K BASIN INTEGRATED \\ WATER TREATMENT SYSTEM
}

\section{Prepared By:}

Los Alamos Technical Associates, Inc.

Richland, WA

$$
\text { For: }
$$

WESTINGHOUSE HANFORD COMPANY

Richland, WA

July 1996 


\section{NOTICE}

This report was prepared as an account of work sponsored by the United States Government and not for the purpose of reliance by any third party. Neither the United States nor the Department of Energy, nor any of the contractors subcontractors, or their employees, make any warranty, express or implied, or assume any legal liability or expressed responsibility for the accuracy, completeness, or usefulness of any information, apparatus, product or process disclosed or represent that its use would not infringe privately-owned rights. Reference herein or any specific commercial product, process, or service by trade name, mark, manufacture, or otherwise, does not necessarily constitute or imply its endorsement, recommendation, or favoring by the United States Government or any agency thereof nor any contractor, subcontractor or their employees. The views and opinions expressed herein do not necessarily state or reflect those of the United States Government or any agency thereof nor any contractor, subcontractor or their employees. Use of any part of this report shall be at the user's sole risk and shall constitute a release and agreement to defend and indemnify the United States, the Department of Energy and/or any of their employees, or any contractor, subcontractor and/or their employees, against any and all liability in connection therewith, regardless of fault or negligence. 
WHC-SD-SNF-RPT-012, Rev. 0

\section{EXECUTIVE SUMMARY}

This report summarizes the status of the Integrated Water Treatment Project design

at the Preliminary Design stage. The scope of work is based on the Statement of Work for Preliminary Design. The purpose of the preliminary design phase was to update the conceptual design, originally presented in March 1996, to reflect changes which resulted from a value engineering study held in May 1996. This Preliminary design report fulfills the requirements of the July 8,1996 milestone.

The main changes which resulted from the value engineering study included; reduction in assumed leach rate from fuel in $K$ East (KE) and a corresponding reduction in the number of ion exchange modules (IXMs) required, use of a commercially available skid mounted backflushable filter unit of smaller overall dimensions than the Conceptual Design Report design location of filter unit in the KE chiller bay, change of the building type for the KE annex to a non-permanent structure, use of flexible hose instead of hard pipe below water, use of single wall pipe instead of double wall pipe, maximizing the use of existing recirculation piping, not modifying the existing skimmer loop, minimal modifications to the existing demineralized water system, removal of new filters from the $K$ West $(K W)$ design and simplification of the KW design, relocation of KW IXMs in transfer bay, and deletion of the requirement for an annex at $\mathrm{KW}$.

During preliminary design the Process Flow Diagrams and material balances were updated. The facility layouts were also modified to reflect the design changes. The preliminary hazards assessment was updated and modified to reflect the latest design. A preliminary project design and construction schedule was prepared and finally a preliminary design estimate was prepared reflective of the latest design and schedule. 
WHC-SD-SNF-RPT-012, Rev. 0

Overall, the design presented with this report represents a sound basis for the start of definitive design. 


\section{CONTENTS}

1.0 INTRODUCTION $\ldots \ldots \ldots \ldots \ldots \ldots \ldots \ldots \ldots \ldots \ldots \ldots \ldots \ldots \ldots$

2.0 VAlue engineERING Recommendations $\ldots \ldots \ldots \ldots \ldots \ldots \ldots$

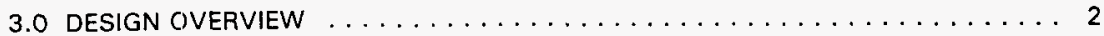

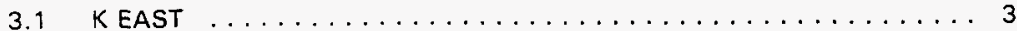

3.1 .1 Main Basin Recirculation .................. 3

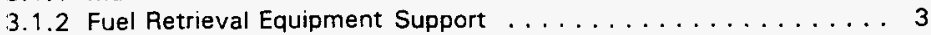

3.1 .3 Weasel Pit ......................... 4

3.1 .4 Skimmer Loop ... . . . . . . . . . . . . . . . . . 4

3.1.5 Water Supply and Demineralized Water Make-Up . . . . . . . . 4

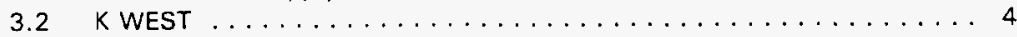

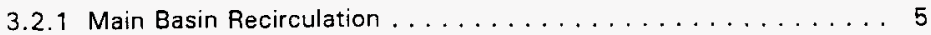

3.2.2 Canister Discharge Treatment . . . . . . . . . . . . . 5

3.2.3 Fuel Retrieval Equipment Support . . . . . . . . . . . . 5

3.2 .4 Recirculation by the Skimmer Loop . . . . . . . . . . . . 6

3.2 .5 Water Supply . . . . . . . . . . . . . . . . 6

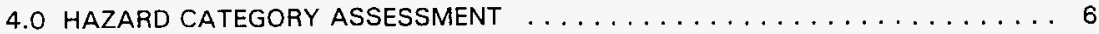

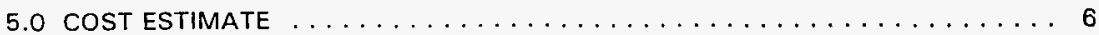

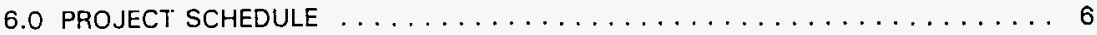

\section{APPENDICES}

APPENDIX A DRAWING PACKAGE $\ldots \ldots \ldots \ldots \ldots \ldots \ldots \ldots \ldots \ldots \ldots$

APPENDIX B HAZARDS CATEGORY CONFIRMATION POSITION PAPER . . . . . . B-1 APPENDIX $\mathrm{C}$ COST ESTIMATE $\ldots \ldots \ldots \ldots \ldots \ldots \ldots \ldots \ldots \ldots \ldots \ldots \ldots \ldots \ldots$

APPENDIX D PROJECT SCHEDULE $\ldots \ldots \ldots \ldots \ldots \ldots \ldots \ldots \ldots \ldots \ldots$ 


\section{ACRONYMS}

$\begin{array}{ll}\text { CDR } & \text { Conceptual Design Report } \\ \text { DOE } & \text { U.S. Department of Energy } \\ \text { FRS } & \text { Fuel Retrieval System } \\ \text { gpm } & \text { Gallons per minute } \\ \text { IWTS } & \text { Integrated Water Treatment System } \\ \text { IXM } & \text { lon Exchange Module } \\ \text { KE } & \text { K East } \\ \text { kg } & \text { Killogram } \\ \text { KW } & \text { K West } \\ \text { MCO } & \text { Multi-Canister Overpack } \\ \text { Mg } & \text { Megagram } \\ \text { PFD } & \text { Process Flow Diagrams } \\ \text { VE } & \text { Value Engineering }\end{array}$




\subsection{INTRODUCTION}

This report summarizes the changes to the $K$ Basins Integrated Water Treatment System (IWTS) Conceptual Design Report (CDR), issued in March 1996. The CDR identified $K$ East and $K$ West system requirements and completed initial Process Flow Diagrams (PF[s). In addition, initial facility layouts were developed which included annex facilities to both basins. A preliminary hazards assessments was included for the purpose of identifying hazard categories and safety classification of the IWT systems, structures, and components. Finally, project cost estimates and schedules were aiso included in the CDR.

In May 1996, a Value Engineering (VE) Session was conducted with the purpose of identifying high costs associated with the CDR design basis and identifying cost saving proposals which, if implemented, would result in a minimum risk to the workers, public, and environment. Eleven cost saving recommendations were presented by the VE team to WHC and DOE management. These recommendations, as approved, would provide a basis for revising or issuing new PFDs, general arrangement layout drawings, hazard category assessment, project cost estimate and project schedule. Using the $11 \mathrm{VE}$ recommendations as specified in the Statement of Work, new flow sheets, PFDs, general arrangement and layout drawings were prepared. Based on these drawings, a new project cost was estimated and a design and construction schedule prepared.

\subsection{VALUE ENGINEERING RECOMMENDATIONS}

The following 11 recommendations were presented by the VE team at the conclusion of the VE session.

- Use of flexible non-metallic pipe instead of hard piping for some applications in the basin. The identified areas for use were for underwater piping from the fuel cleaning equipment, sludge and debris removal equipment.

- Use of single wall piping in the basins in lieu of double wall piping. New piping installed in the basins would be single wall (similar to existing basin piping) and only piping installed outside the basin and annex would require double containment.

- Maximize use of existing piping. The existing piping for the main recirculation suction and discharge piping would be used.

- Provide all necessary flow distributions. The water usage from the various sub-projects would be revised to reflect current needs. (Note: the current overall water requirements are greater than those used during CDR development and result in a cost addition instead of a savings. However, the new flow distribution satisfies the current sub-projects needs.) 
- Revise $K$ East (KE) ion exchange design basis. The total flowrate through ion exchange modules (IXMs) and hence, the total number of required IXMs would be reduced due to: (1) a lower anticipated ${ }^{137} \mathrm{Cs}$ leach rate, and (2) allowable worker dose rate increasing from 0.5 to $1 \mathrm{rem} / \mathrm{yr}$. The ${ }^{137} \mathrm{Cs}$ concentration goal in the basin would increase to $1 \mu \mathrm{Ci} / \mathrm{L}$ from $0.5 \mu \mathrm{Ci} / \mathrm{L}$. The use of ion specific resins (i.e., zeolites) would not be used, only mixed-resin bed IXMs would be used.

- Revise K West (KW) IWTS design. The VE recommendation is to reduce the total number of processing loops from three to two, use only mixed bed IXMs, eliminate pre-filters on the fuel processing loop, keeping the existing cartridge filters as is, and reducing the number of required IXMs from five to three.

- Retrain sand filters and operate the skimmer loop as is. The use of sand filters retains 400 gallons per minute $(\mathrm{gpm})$ of filtration capacity. Deleting the skimmer loop piping modification would eliminate piping rework.

- Revise allowances for mask work, burnout, mockups and training. This recommendation does not change the CDR design basis but changes the project costs and schedule.

- Change Annex Building type to a non-permanent structure. The CDR recommended a metal building with internal shieid walls. The VE recommendations is to use a pre-engineered modular type, metal building resulting in quicker erection and take down.

- Maximize use of existing facilities and simplify the annex. The VE recommendation is to use a small, compact filter skid in the KE Basin as opposed to a larger unit. This would eliminate the need for locating the filters in the annex, saving on annex size. The filter skid would be the same or similar to a unit supplied by PN Services and would be located in the KE Chiller Bay.

- Eliminate the KW Annex. The sixth VE recommendation reduced the number of required IXMs from five to three and eliminated the need for prefiltration of the fuel processing loop. Therefore, sufficient space exists in the $\mathrm{KW}$ north transfer bay for locating the require IXMs, eliminating the need of an annex.

\subsection{DESIGN OVERVIEW}

The VE design changes are reflected in the revised PFDs and facility layouts which are included as Appendix $A$. The design is summarized below. 


\subsection{K EAST}

The original water treatment proposals have been modified to include changes to the five main sub-systems of: (1) Main Basin Recirculation, (2) Fuel Retrieval Equipment Support, (3) Weasel Pit, (4) Skimmer Loop, and (5) Water Supply and Demineralized Water Make-Up.

\subsubsection{Main Basin Recirculation}

The design utilizes the existing basin water offtake piping but will replace the existing recirculation pumps with three new $500 \mathrm{gpm}$ units located in the existing pump positions. Fire particle filtration is now undertaken in two skid mounted backflush filter units located within a contamination controlled area in the main basin Chiller bay. The filter skids are self shielded and are positioned in close proximity to the weasel pit minimizing the backflush pipe route. New eight inch (in.) single-wall piping is routed from the main loop to the new filter skids. Existing valved chiller connections are utilized to tie the filtered water back into the existing main loop for routing to the chiller system and back to the basin. Filter backflush is routed via a shielded pipeline and surge tank to the east end of the Weasel pit. Filter change-out will utilize a semi-remote shielded transfer system requiring close operational control. Spent filter units will be deposited in a shielded container and will be fully monitored before removal from the containment room and basin. Filter container export will be through the east exit door in the Chiller bay. The existing filtration loop is retained within the system to provide additional coverage if required.

Ion Exchange is provided by three mixed beds, one Anion and one spare IXM located in a rew, temporary, 1500 feet $^{2}(\mathrm{ft})$ low-height annex facility located on the existing radwaste storage pad north of the basin. The existing $1 X M$ design is retained but with internal piping and filling connections modified to allow system disconnection from a low platform. Access to the annex is provided via a short double-door link corridor and provides an area for a step-off-pad. All piping and drain connections to the annex are through the link corridor satisfying double containment requirements. All piping routes within the annex are single wall and utilize valved hose connections to the IXMs. Other main features of the annex include; IXM change out using a tractor controlled air pallet system; a bermed area with drainage to the basin; a water sampling system; sump system with alarm; an air compressor to supply the air pallet, filter, and instrumentation; and a heating system.

\subsubsection{Fuel Retrieval Equipment Support}

To support VE recommendations, all in water pipe routes from the fuel retrieval equipment will use flexible nonmetallic hoses supported on flotation devices. The hoses will be routed four feet below the surface of the water to avoid interference with fuel retrieval and provide radiation protection for operation personnel. Water treatment equipment will include the two primary clean and down draft table discharge pumps. Connections to underwater equipment are single handed, quick-connect/disconnect hose couplings to aid installation and hose change out if required. 
A twc-inch hose is routed along the south side of the basin from an $80 \mathrm{gpm}$ submersible fump mounted on the fuet retrieval primary clean equipment to a hydrocyclone mounted in a shielded module above the east end of the weasel pit. Heavy sludge particles will be discharged directly to the pit with the cleaner water discharged to a weir system located in the west end, for forward routing to the recirculation system. The sludge will initially be deposited in the weasel pit east end. As more sludge is transferred to the pit, it will be necessary to utilize the west end for studge staging.

A four in. hose is routed parallel to the primary-clean hose from a $420 \mathrm{gpm}$ submersible pump mounted on the fuel retrieval down-draft table to the recirculation pump header.

\subsubsection{Weasel Pit}

A three in. hose is routed in parallel to the primary clean and down-draft hose routes from sludge removal equipment to a series of hydro-cyclones mounted alongside the primary clear hydrocyclone in the weasel pit. Discharge routes are the same as the primary clear description. The weasel pit is isolated from the basin by a three section seal plate which prevents contaminated water and sludges from dispersing into the main basin. The weasel pit weir system will control the pit water level to approximately one ft below the main basin to provide a hydraulic differential into the pit.

\subsubsection{Skimmer Loop}

No modifications are proposed to the existing skimmer loop system.

\subsubsection{Water Supply and Demineralized Water Make-Up}

Treated water is supplied to maintain water level in the basin and to supply water to the fuel retrieval, multi-canister overpack (MCO)/cask loadout, discharge chute purge, sludge removal and debris removal projects.

Due to the position of most of these supplies, all piping will be hard piped with the exception of a two inch hose supply to the fuel retrieval secondary cleaning equipment.

\subsection{K WEST}

The original water treatment proposats have been modified to include changes to the five main subsystems: (1) Main Basin Recirculation, (2) Canister Discharge Treatment, (3) Fuel Retrieval Equipment Support, (4) Recirculation by the Skimmer Loop, and (5) Water Supply. In addition, return water is added for the debris removal enclosure. 


\subsubsection{Main Basin Recirculation}

The Main Basin Recirculation consists of basin water that is recirculated at a nominal $970 \mathrm{gpm}$ flow rate using two new pumps and the existing offtake piping. The Fuel Retrieval System (FRS) supplies $420 \mathrm{gpm}$ from the downdraft table and damaged canister staticn and Sludge Removal Sub-Project supplies $100 \mathrm{gpm}$. The Debris Removal Sub-Project also supplies $100 \mathrm{gpm}$ in an intermittent flow. The lines from FRS and Sludge Removal will both use flexible non-metallic hose to transfer the water to a position close to the pump suctions into hard piping at a depth of approximately 4 feet below the surface. A new submersible pump will be installed by IWTS to interface with the FRS. The combined $970 \mathrm{gpm}$ flow is then recirculated using two new centrifugal pumps and a new spare pump.

The main recirculation to the existing cartridge filters is maintained within the system. These five-micron, nominally rated, filters are used to: (1) remove particulates maintaining water clarity, (2) remove insoluble radionuclides, and (3) protect the downstream ion-exchange beds. The existing air-cooled exchanger maintains the basin at the required temperature. Approximate $225 \mathrm{gpm}$ is pumped through the exchanger prior to recombining with the main stream.

Part of the $970 \mathrm{gpm}$ filter discharge stream is diverted to three IXMs relocated to the North Transfer Bay. The three IXMs operating in parallel configuration can process about $480 \mathrm{gpm}$ of basin water keeping the system in a demineralized state and providing high purity water to other Spent Nuclear Fuel Sub-Projects. The six-inch discharge from these IXMs ties into the existing skimmer loop system near the north loadout pit, to provide a return to the basin unless otherwise directed to the sub-projects. The main recirculation loop water that does not divert to the IXMs then discharges into the basin on the south side through the three existing submerged discharge nozzles, one located in each bay.

\subsubsection{Canister Discharge Treatment}

The IX.Ms also receive and treat water from the canister decapping equipment used by the FRS. Water is drawn from the inside and surrounding area of a canister during the delidding process. Water is pumped at approximately $150 \mathrm{gpm}$ in a three-inch hose from the delidding station within the basin. Hoses will be used within the basin to transfer the water to the edge of the basin where it will tie into hard piping below the water surface.

\subsubsection{Fuel Retrieval Equipment Support}

The two in. discharge from the delidding equipment is combined with approximately $80 \mathrm{gpm}$ from the three-inch FRS primary cleaning station. Hoses will be used within the basin to transfer the water to the edge of the basin. The combined $230 \mathrm{gpm}$ flow discharges into a three in. pipe and then combined with the main recirculation loop six-inch pipe and is directed to the IXMs. 


\subsubsection{Recirculation by the Skimmer Loop}

The existing skimmer loop is retained for coarse filtration.

\subsubsection{Water Supply}

The requirements for water supply are similar to those for the KE Basin. Make-up water is required to maintain the water level in the pool and to supply water to the Fuel Retrieval, MCO/Cask Loadout, Sludge Removal, and Debris Removal Sub-Projects.

Fresh rnake-up water is required to offset basin water loss due to evaporation, removal of fuel and sludge, and possible leakage. In addition, fresh demineralized water (at $<0.2 \mu$ mhos ${ }^{\prime} \mathrm{cm}$ ) is required to rinse the MCOs upon removal from the basin. The basin currently has a demineralized system to supply basin make-up. The existing system is rated at $12 \mathrm{gpm}$. Minor modifications are required to the existing system.

High purity, low conductivity treated water is also required to support the basin MCO/Cask Loadout. A four in. line is provided from the IXM discharge to the south load out pit, utilizing existing piping as much as possible.

\subsection{HAZARD CATEGORY ASSESSMENT}

The hezard categorization from the CDR was updated to reflect the design modifications which resulted from the value engineering study. The revised assessment is included in Appendix $B$.

\subsection{COST ESTIMATE}

A preliminary design estimate has been prepared and is included in Appendix C.

\subsection{PROJECT SCHEDULE}

A project design and construction schedule has been prepared and is included in Appendix D. 


\section{APPENDIX A}

\section{DRAWING PACKAGE}

Drawing List:

H-1-81984, Rev. B, 105 KE Process Flow Sheet H-1-81985, Rev. B, 105 KW Process Flow Sheet H-1-81986, Rev. B, 105 KE Process Flow Diagram H-1-81987, Rev. B, 105 KW Process Flow Diagram $\mathrm{H}-1-82014$, Rev. B, $105 \mathrm{KE}$ General Arrangement H-1-82015, Rev. B, 105 KE IXM Annex Layout $\mathrm{H}-1-82016$, Rev. B, 105 KE Chiller Bay Piping Plan $\mathrm{H}-1$-82017, Rev. B, 105 KE Weasel Pit Arrangement H-1-82018, Rev. B, $105 \mathrm{KW}$ Main Basin and Transfer Bay Layout 


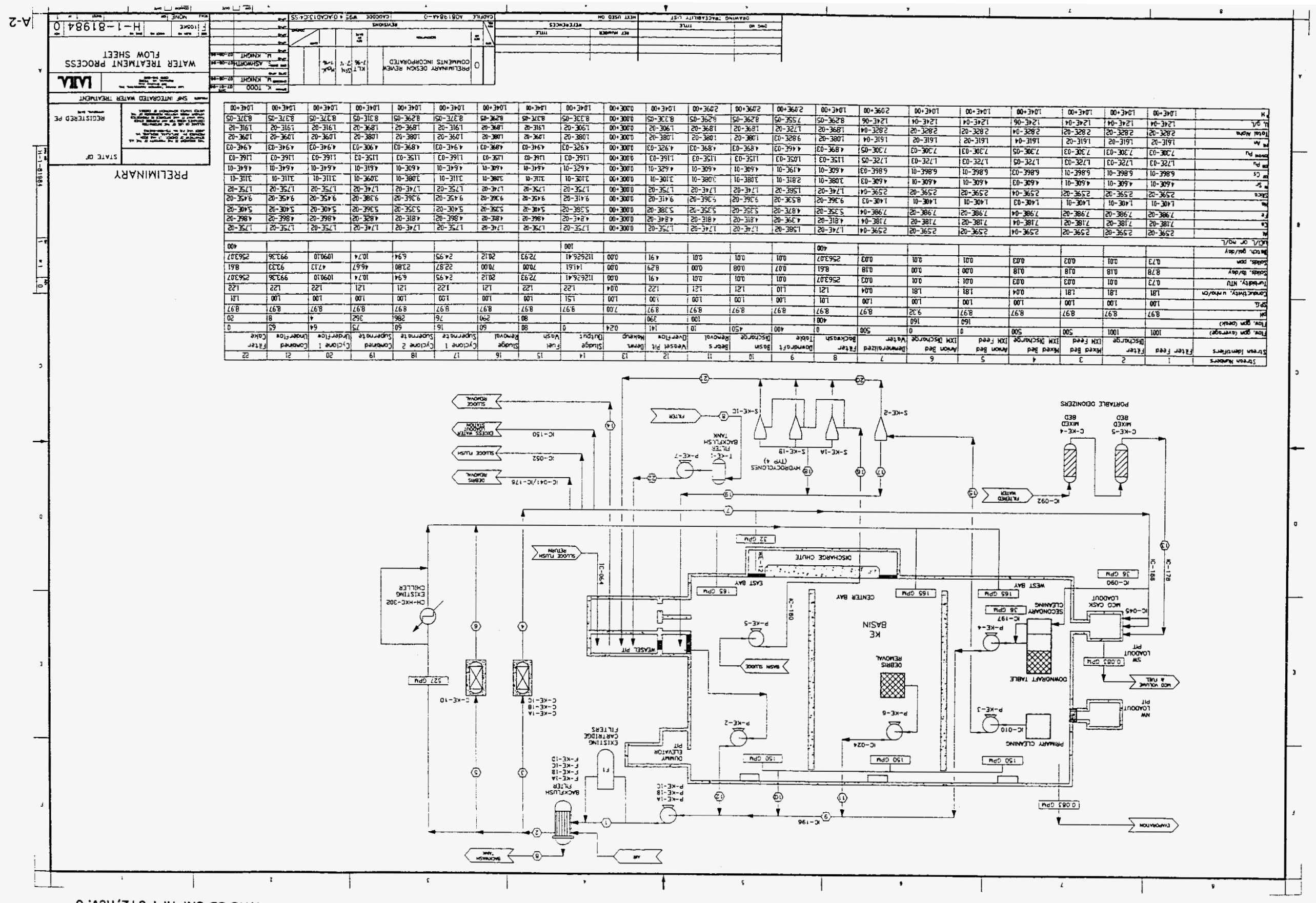

0 กөy ' $Z$ LO- $-19 y-3 N S-O S-J H M$ 


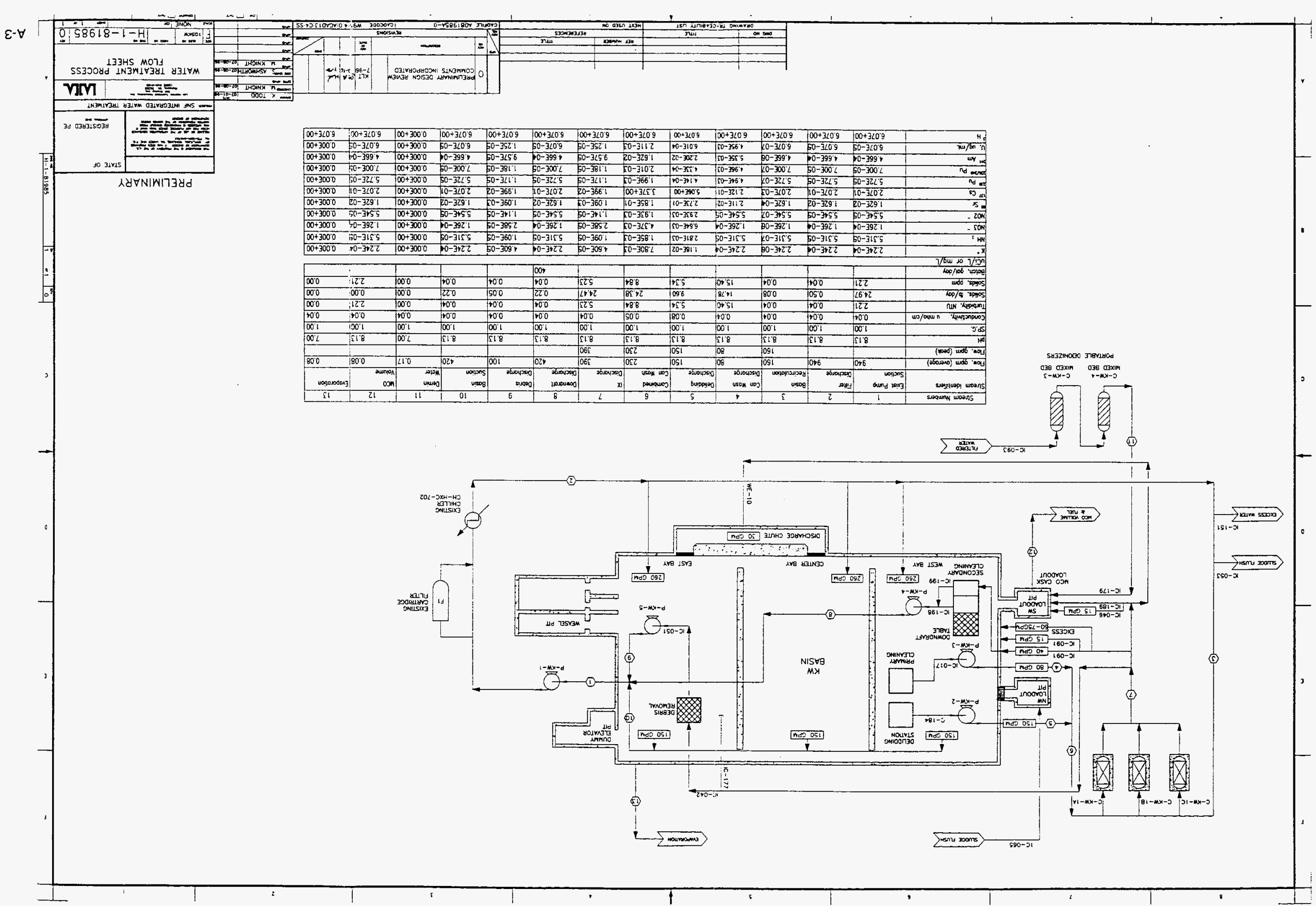

$0 \cdot \wedge \theta y^{\prime} Z L O-\perp d y-J N S-O S-J H M$ 


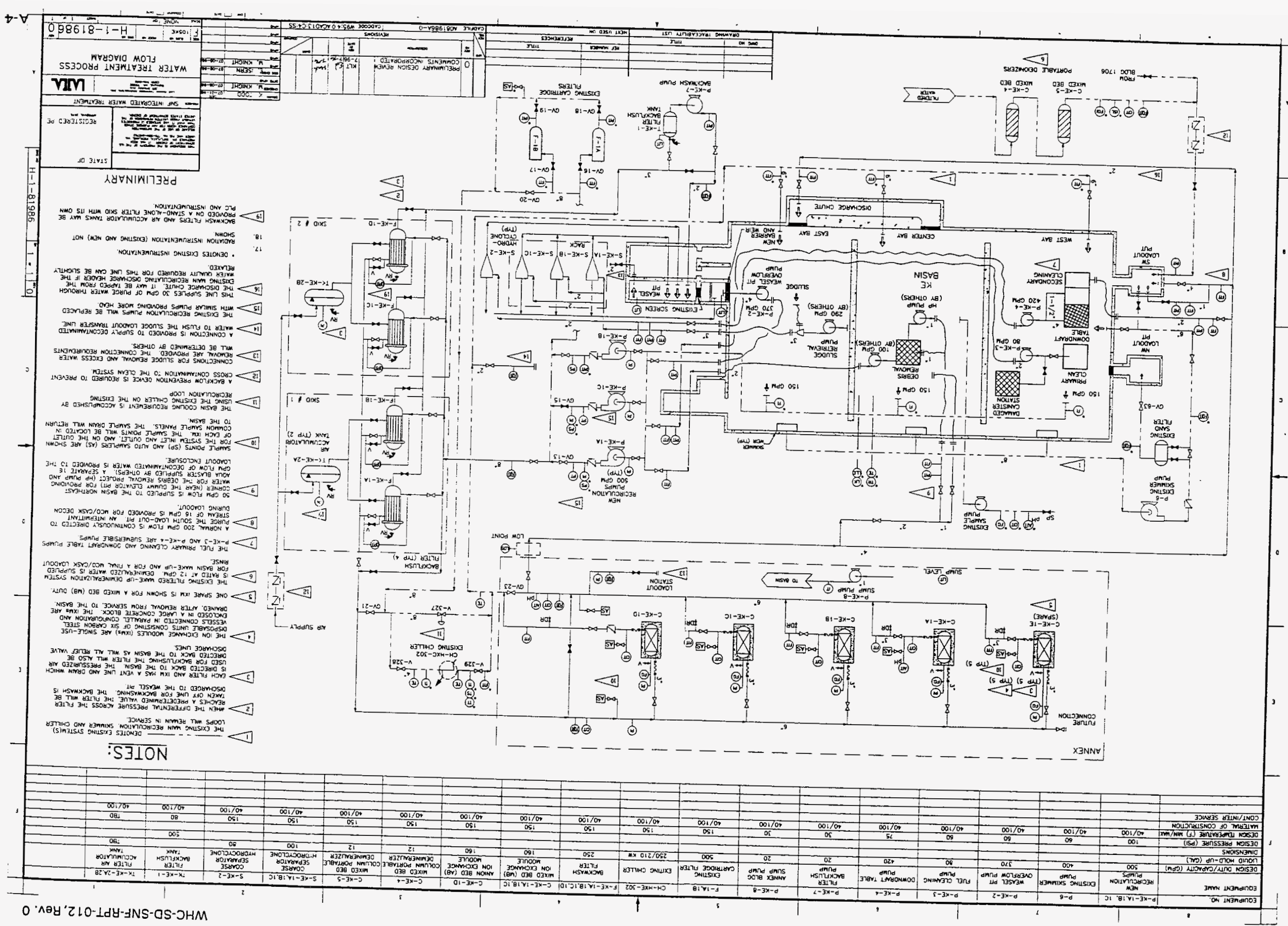




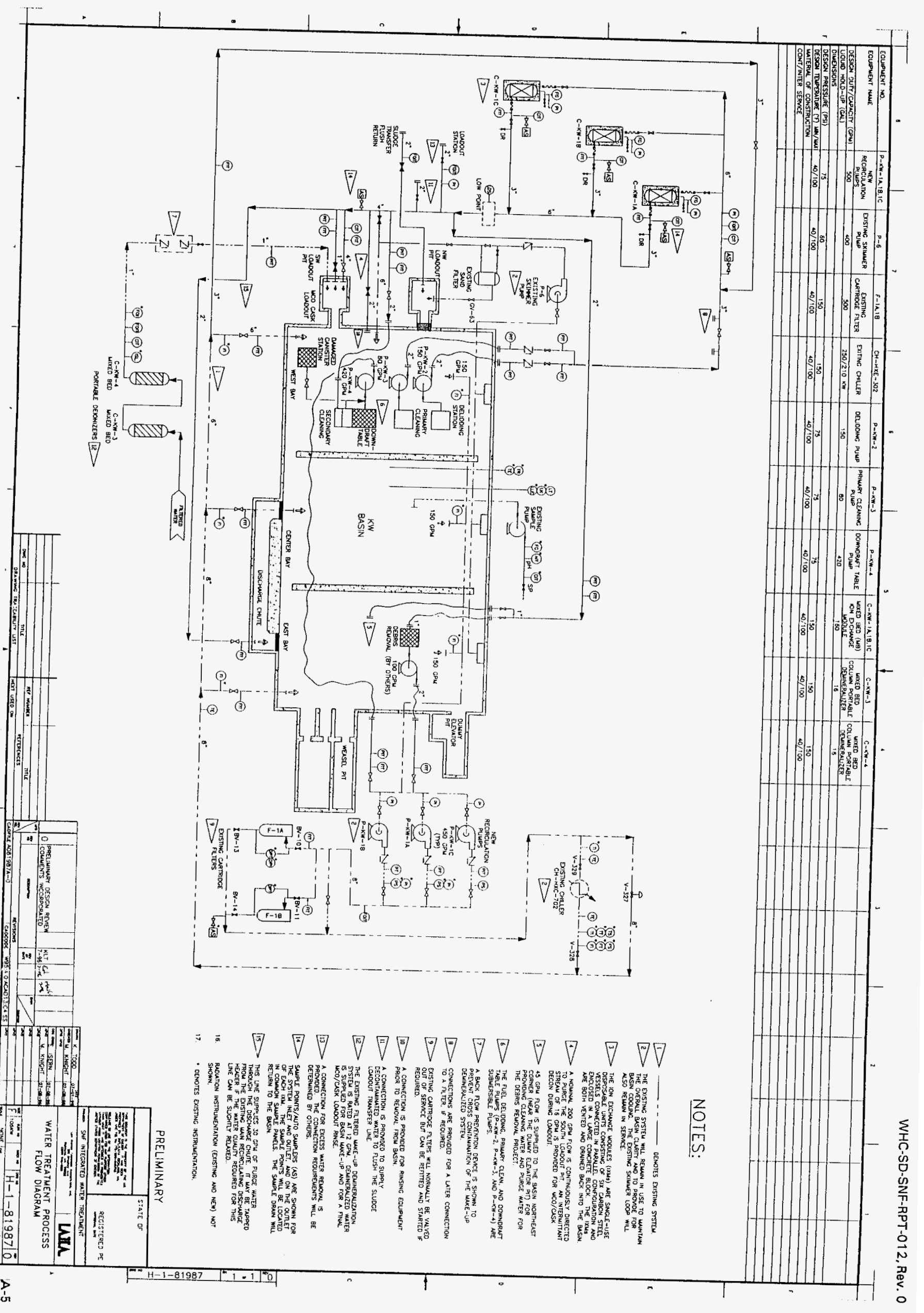




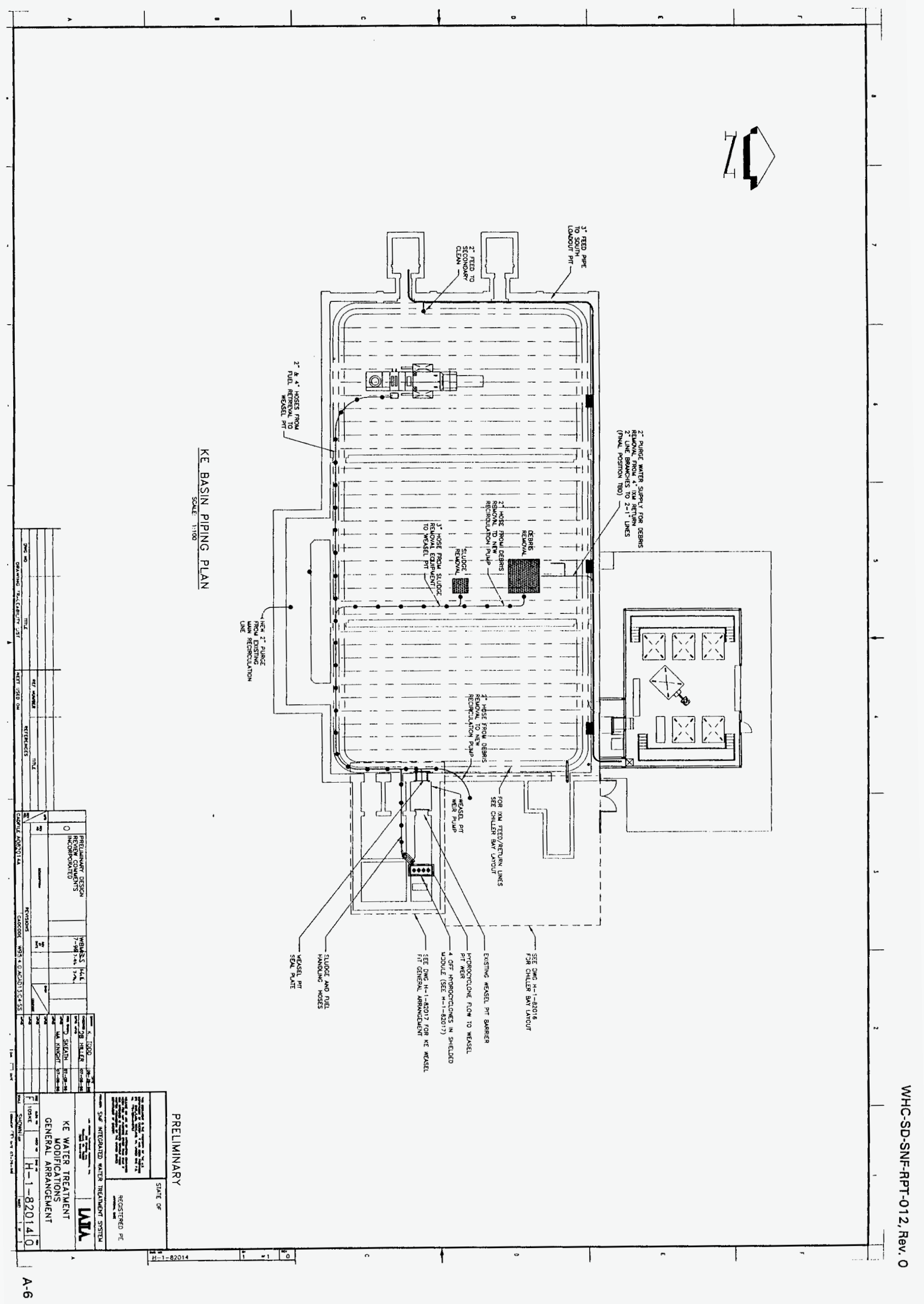




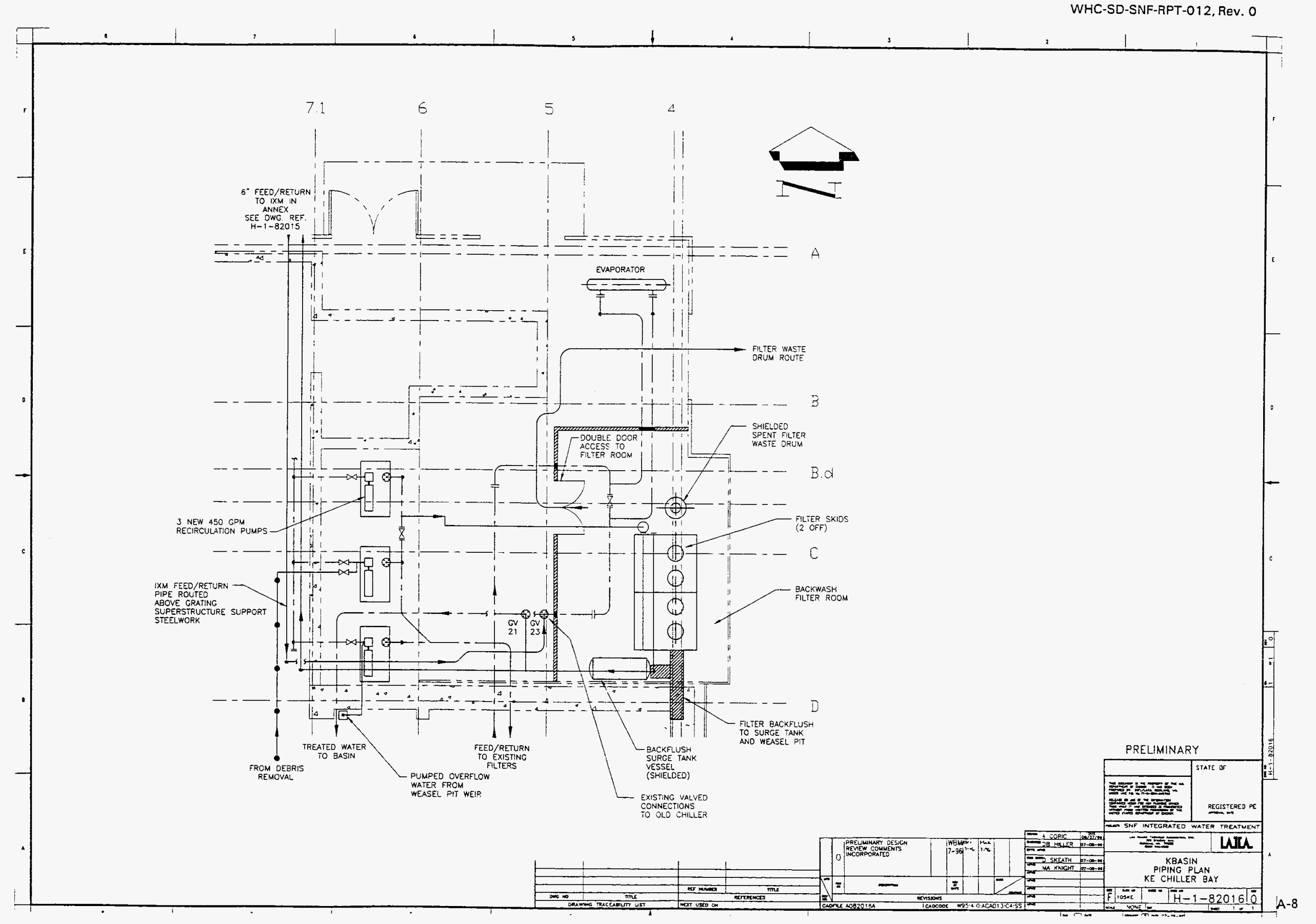




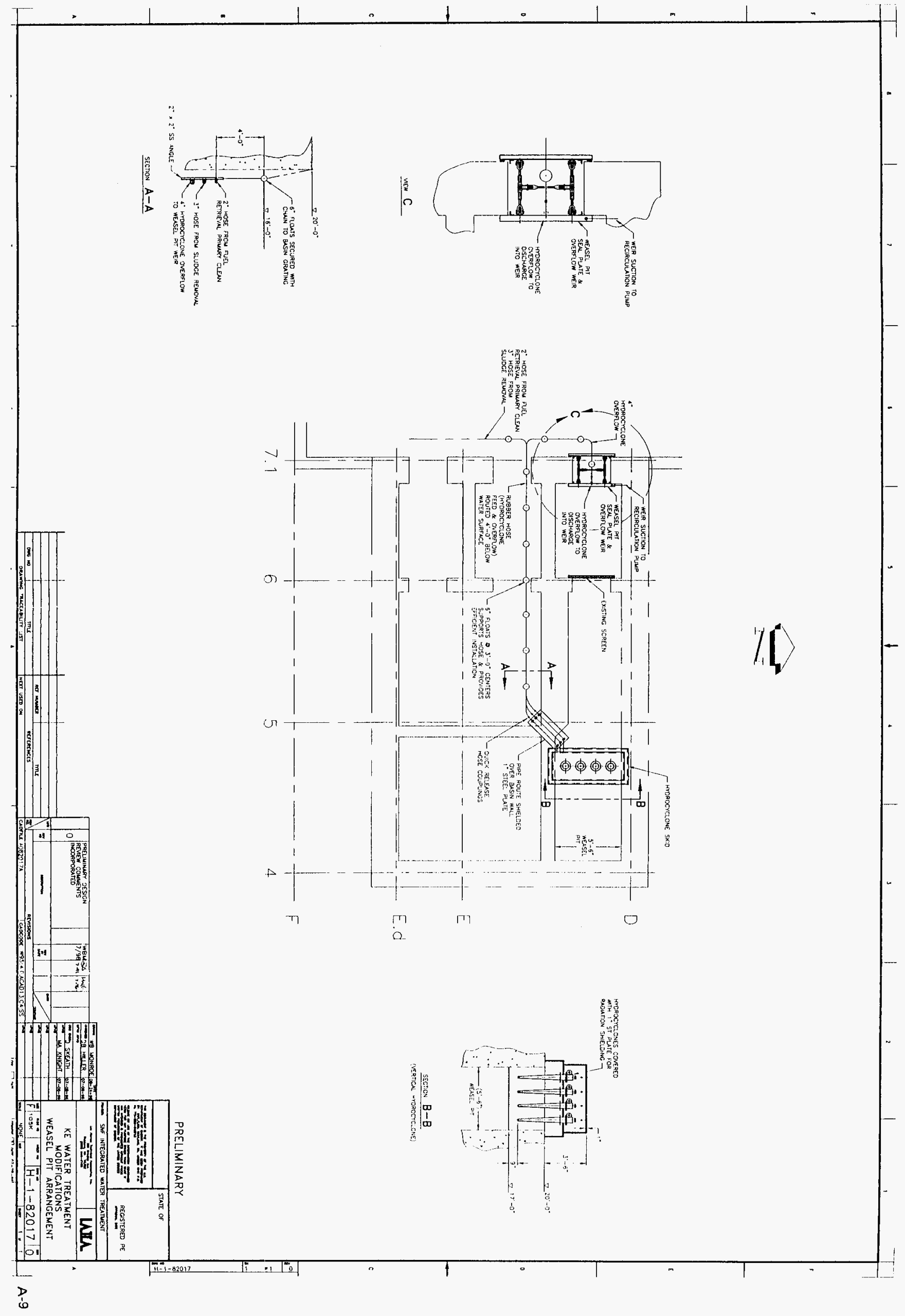




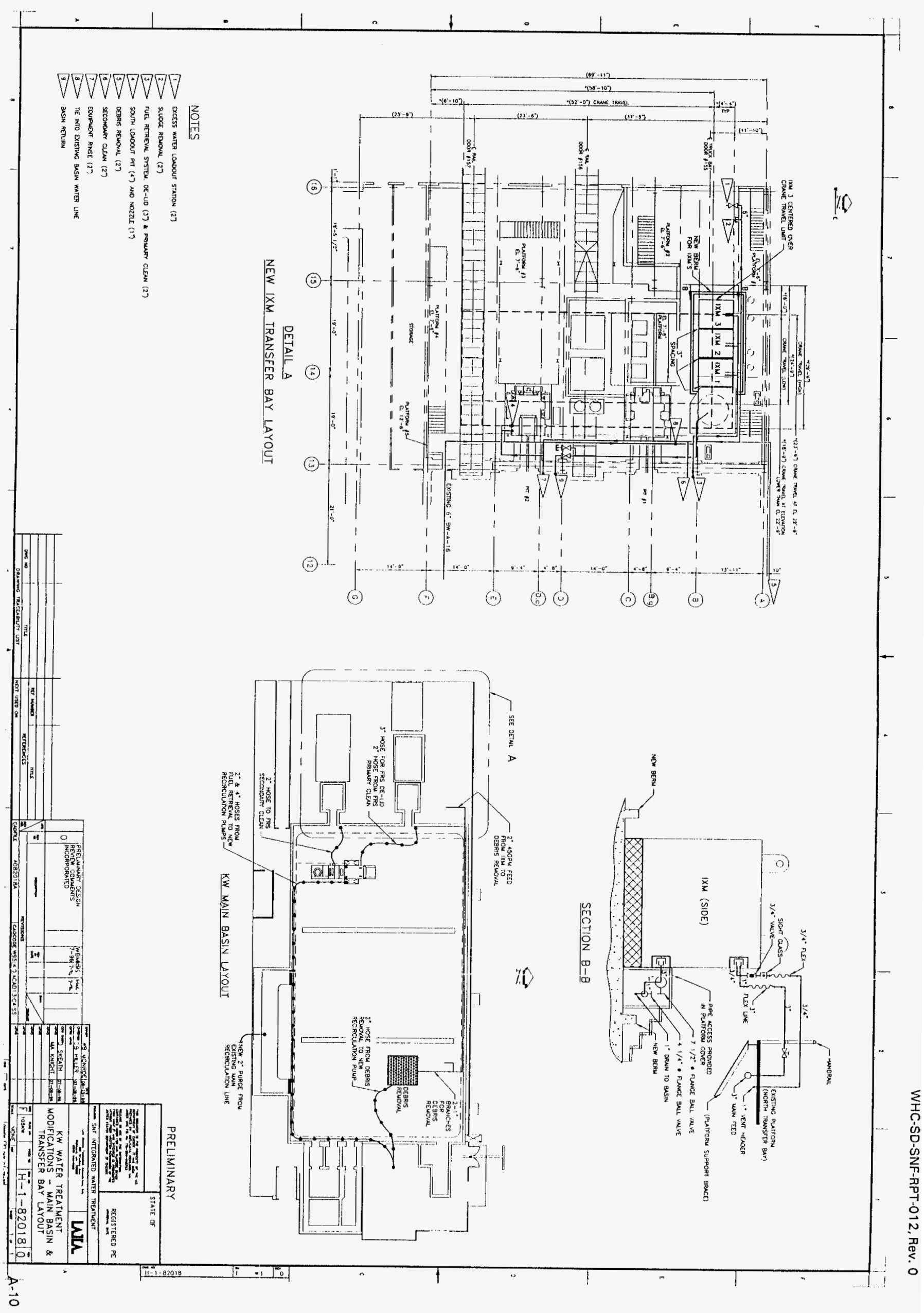


WHC-SD-SNF-RPT-012, Rev. 0

\section{APPENDIX B}

\section{HAZARDS CATEGORY CONFIRMATION POSITION PAPER}

B - i 
WHC-SD-SNF-RPT-012, Rev. 0

\section{K BASIN INTEGRATED WATER TREATMENT SYSTEM}

PRELIMINARY HAZARD CATEGORIZATION IN SUPPORT OF THE PRELIMINARY DESIGN

Prepared By:

Los Alamos Technical Associates, Inc.

Richland, WA

For:

WESTINGHOUSE HANFORD COMPANY

Richland, WA

July 1996

Bii 


\section{CONTENTS}

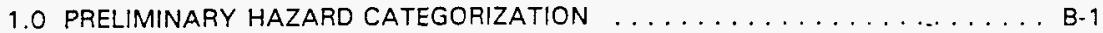
1.1 HAZARD CATEGORIZATION APPROACH FOR THE K BASINS INTEGRATED WATER TREATMENT SUB-PROJECT . . . . . . . . B B-2

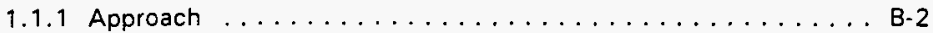
1.1 .2 Criticality Sensitivity $\ldots \ldots \ldots \ldots \ldots \ldots \ldots$

$2.0 \mathrm{~K}$ EAST INTEGRATED WATER TREATMENT PROJECT . . . . . . . . . B-3

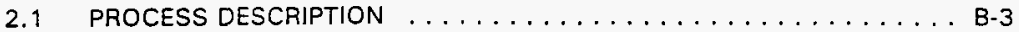

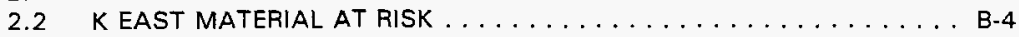

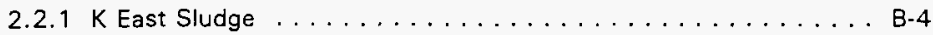

2.2.2 K East Canister Sludge . . . . . . . . . . . . . . B B-4

2.2.3 K East and $\mathrm{K}$ West Fuel Assemblies . . . . . . . . . . . . . B-4

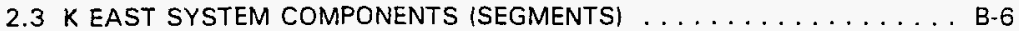

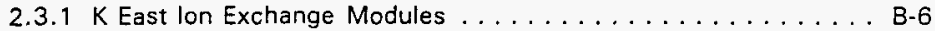

2.3.2 Hydrocyclones . . . . . . . . . . . . . . . . . B-6

$2.3 .3 \mathrm{~K}$ East Filter Vessets . . . . . . . . . . . . . . . B-6

2.3.4 K East Filter Backwash Tank . . . . . . . . . . . . . . B-7

$2.3 .5 \mathrm{~K}$ East Submerged Filters ................... .

$3.0 \mathrm{~K}$ WEST INTEGRATED WATER TREATMENT PROJECT $\ldots \ldots \ldots \ldots \ldots \ldots \ldots$

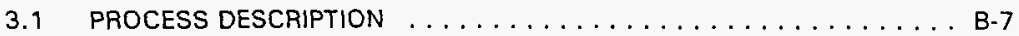

3.2 KWEST MATERIAL AT RISK $\ldots \ldots \ldots \ldots \ldots \ldots \ldots \ldots \ldots \ldots$

3.2.1 K West Main Basin Sludge . . . . . . . . . . . . . . B-8

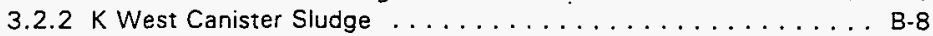

$3.2 .3 \mathrm{~K}$ West Fuel Assemblies . . . . . . . . . . . . . . . B-8

$3.3 \mathrm{~K}$ WEST SYSTEM COMPONENTS (SEGMENTS) . . . . . . . . . . B-8

3.3.1 $\mathrm{K}$ West lon Exchange Modules . . . . . . . . . . . . B-8

3.3.2 K West Submerged Filters . . . . . . . . . . . . B-8

4.0 K EAST BOUNDING SCENARIOS FOR HAZARD CATEGORIZATION . . . . . . . B-9

4.1 K EAST HAZARD CATEGORIZATION CONCLUSION . . . . . . . . B-10

$5.0 \mathrm{KW}$ BOUNDING SCENARIOS FOR HAZARD CATEGORIZATION . . . . . . . . B-10

$5.1 \quad K$ WEST HAZARD CATEGORIZATION CONCLUSION . . . . . . . . . B 11

6.0 INSTALLATION RISKS $\ldots \ldots \ldots \ldots \ldots \ldots \ldots \ldots \ldots \ldots \ldots \ldots \ldots$

7.0 REFERENCES $\ldots \ldots \ldots \ldots \ldots \ldots \ldots \ldots \ldots \ldots \ldots \ldots \ldots \ldots \ldots$

ATTACHMENT A RADIOLOGICAL INVENTORIES FOR B-Attach-1 105-K BASINS 
WHC-SD-SNF-RPT-012, Rev. 0

\section{LIST OF TABL.ES}

1. Comparison of K Basin Segments Of IWTP to Category 2 Threshold Quantity Values ......................... B 
WHC-SD-SNF-RPT-012, Rev. O

PRELIMINARY HAZARD CATEGORIZATION DUE TO VALUE ENGINEERING SESSION

SUMMARY: The following Preliminary Hazard Categorization for the KE and KW Integrated Water Treatment Project (IWTP) determines that both should be designated as Hazard Category 3.

\subsection{PRELIMINARY HAZARD CATEGORIZATION}

Hazard Categorization is the evaluation of the consequences of unmitigated releases to classify facilities or operations into the following hazard category definitions and interpretations provided in DOE-STD-1027-92, Hazard Categorization and Accident Analysis Techniques for Compliance with DOE Order 5480.23, Nuclear Safety Analysis Reports, DOE (1992a):

\section{DEFINITION}

Hazard Category 1: The hazard analysis shows the potential for significant offsite consequences.

\section{INTERFRETATION}

Category $A$ reactors and facilities designated by the Program Secretarial Officer

\section{DEFINITION}

Hazard Category 2: The hazard analysis shows the potential for significant onsite consequences.

\section{INTERFRETATION}

Facilities with the potential for nuclear criticality events or with sufficient quantities of hazardous material energy, which require on-site emergency planning activities.

\section{DEFINITION}

Hazard Category 3: The hazard analysis shows the potential for only significant localized consequences.

\section{INTERFRETATION}

Facilities with quantities of hazardous materials which meet or exceed the Threshold Quantity (TQ) 3 values shown in DOE 1992a.

A preliminary Hazard Categorization is intended to be a cursory exercise to categorize a U.S. Department of Energy (DOE) nuclear facility using basic facility information on intended operations and estimates of radioactive material quantities. Whenever questions concerning appropriate facility categorization arise, DOE (1992a) requires providing a margin for error by selecting the higher hazard category. A Hazard Categorization is based on an "unmitigated" release of available hazardous material. "Unmitigated" is meant to consider material quantity, form, location, dispersibility and interaction with available energy sources, but not to consider safety features (e.g. ventilation system, fire suppression, etc.) which will prevent or mitigate a release. 
Many DOE operations conduct a wide variety of activities in one facility, ranging from simple assay or lab experiments to complex fluid separations. For this reason, (DOE 1992a) allows facility segmentation. This is atlowed where features inherent to the facility preclude bringing material together, including harmful interaction from a common severe phenomena (DOE 1992a). DOE Order 5480.23 (DOE 1992b) states that an analysis and categorization is to be performed on "processes, operations, or activities" and not necessarily whole facilities. DOE-STD-1027-92 provides guidance on determination of hazard categories based on comparison of individual radionuclides to the threshold quantities for Hazard Category 2 and 3 . The ratios between the facility inventory material at risk and the category threshold quantity for each radionuclide are summed. If each individual quantity is less than the threshold quantity and the sum of the ratios for all nuclides are less than the threshold quantity, the facility is categorized at the lower categorization.

\subsection{HAZARD CATEGORIZATION APPROACH FOR THE K BASINS INTEGRATED WATER TREATMENT SUB-PROJECT}

This Preliminary Hazard Categorization considers the IWTP and its interfaces with future SNFP Sub-Projects. The future projects include:

- Fuel Retrieval Sub-Project

- Sludge Retrieval Sub-Project

- Debris Retrieval Sub-Project

- $\mathrm{MCO} /$ Cask Loadout Sub-Project

To perform this preliminary Hazard Categorization, existing knowledge of the above projects are considered in order to determine the potential sources of material the IWTP will need to process. The radioactive sources (material at risk, which are known or estimated to clate) are then investigated as to which ones contribute the most in determining the appropriate Hazard Categorization for the Integrated Water Treatment SubProject (IWTPI. In many cases, the designs of the various Sub-Projects have not matured enough to have definitive material at risk estimates in the form necessary for a Final Hazard Categorization determination, and therefore reasonable yet conservative estimates are made. In many cases, a per unit contribution to the Category 2 TO is made, for example the fraction of the TO 2 value per $\mathrm{kg}$ of sludge. This enables the preliminary categorization to account for uncertainties in the final design features of the IWTP and its interfacing projects, and is consistent with the intended purpose of hazard categorization.

\subsubsection{Approach}

The Hazard Categorization of the IWTP has been updated from the June 25, 1996 , version (LATA 1996) as to recommended source terms (material at risk) and from inputs from meetings among BNFL Inc., Los Alamos Technical Associates (LATA), Westinghouse Hanford Company (WHC), and results of the VE Session (WHC 1996a). The IWTP has not only to consider current K Basins operations, but also future Sub-Projects, such as sludge retrieval, debris removal, and fuel retrieval. This paper, thus, presents an up-date of the approach, assumptions and conclusions for the previous Hazard Categorization, based upon the VE Session, and a more mature design. 
The major recommended system changes from the VE Session and a more mature design in terrns of their effects on the Hazard Categorization of KE and KW IWTP are: (1) elimination of the submerged filters for $\mathrm{KW},(2)$ three filter cells being replaced by a filter skid containing four filter vessels for $K E$, and (3) addition of a filter backwash tank for KE.

The approach taken for the Hazard Categorization of the IWTP includes segmentation of the IWTP processes and components when justified, to more accurately determine hazard categorization. Table 1 shows the relative contribution to a Hazard Category 2 facility and process from each of the segments as if each could physically hold such values. Each of the segments are further discussed in the following paragraphs.

\subsubsection{Criticality Sensitivity}

Table A.1 of DOE-STD-1027-92 shows that the Category 2 TO for two of the isotopes $~^{235} \mathrm{U}$ and ${ }^{239} \mathrm{Pu}$ ) are sensitive to whether the nature of the process precludes the potential for a criticality. With criticality as a concern, the TQ for a Hazard Category 2 facility/process is $1.5 \mathrm{E}-03 \mathrm{Ci}$ for ${ }^{235} \mathrm{U}$, while without criticality concern, the TO is $2.4 \mathrm{E}+02$ $\mathrm{Ci}$. Less sensitive is ${ }^{239} \mathrm{Pu}$, which has a TQ of $2.8 \mathrm{E}+01 \mathrm{Ci}$ with criticality concern, and $5.6 E+01 \mathrm{Ci}$ without criticality concern (DOE 1992). Comparison of the third and fourth columns of Table 1 shows the sensitivity of the hazard category to these varying threshold quantities of fissile materials. Derivation of the values are found in Attachment $A$. For this Hazard Categorization, if documentation does not exist to substantiate that criticality is not a concern, then the lower values are used. If the result does not exceed the TO Hazard Category 2 value of 1 , this shows that potential criticality issues are not of concern to the facility hazard category.

\subsection{K EAST INTEGRATED WATER TREATMENT PROJECT}

\subsection{PROCESS DESCRIPTION}

The KE: IWTP newly installed equipment will consist of four hydrocyclones, four filter vessels, five (four in service, one spare) ion exchange modules (IXMs) and one filter backwash tank. For the Fuel Retrieval Sub-Project, a pump will transfer the outflow from the primary cleaning station (washing machine) and damaged canister station, to one of the hydrocyclones. The underflow (consisting mainly of sludge and solids) of the hydrocyclone will initially discharge to the east section of the Weasel Pit, while the overflow will go to an overflow weir located in the west section. The two sections of the Weasel Pit are separated by a vertical concrete wall, with a 400 mesh screen from about four $\mathrm{ft}$ above the basin floor to the top of the wall. The west end of the west section of the Weasel Pit will use a weir which allows the liquid in the west section of the Weasel Pit to be pumped to the main basin recirculation pumps. The flow from the downdraft table required for the Fuel Retrieval Sub-Project will also be routed to the existing recirculation pump suction header and to the four filter vessels (connected in parallel). The liquid is discharged from the filter vessels with a portion of the flow directed to the four IXMs (connected in parallel) prior to returning to the basin. The solids that accumulate in the four filter vessels will be periodically discharged into a backwash tank; the backwash tank will then be periodically pumped into the east section of the Weasel Pit. 
During the Sludge Retrieval Sub-Project, sludge will be pumped to three hydrocyclones also located in the Weasel Pit east section. The hydrocyclone underflow and overflow for sludge retrieval will then be routed in the same way as the other hydrocyclone.

During the Debris Removal Sub-Project, the outflow of the debris removal enclosure will be pumped to the main basin recirculation pump suction header and to the four filter vessels.

For the MCO/Cask Loadout Sub-Project, only small quantities of radionuclides will need to be processed by the IWTP. This is accomplished by the main basin recirculation loop.

The process flow diagrams for KE are included in Appendix $A$.

\subsection{K EAST MATERIAL AT RISK}

\subsubsection{K East Sludge}

Comparison of KE main basin sludge to Weasel Pit sludge (Tables A-6 through A-9) shows that the Weasel Pit sludge, on a per $\mathrm{kg}$ basis, has a higher contribution to a Hazard Category 2 facility/process than main basin sludge. Canister sludge for KE is considered to be best represented by KE Weasel Pit sludge (Willis 1995). Numerous criticality studies have been performed for KE Weasel Pit sludge and with the current $1 / 4$ inch (in.) filters, criticality has effectively been eliminated (Wittekind 1992, WHC 1995). Furthermore, a 1961 analysis by Neeley and Handler (1961) showed that for homogeneous solutions involving less than $1 \%{ }^{235} \mathrm{U}$, a criticality is not credible. A 1992 analysis by Wittekind, Schwinkendorf and Heer (WHC 1993) showed that for an infinite slab height of Weasel Pit sludge under optimum conditions, the configuration had a $k_{\mathrm{eft}}$ of 0.9743 .

\subsubsection{K East Canister Sludge}

The KE canisters are estimated to contain $3.15 \mathrm{~kg}$ of sludge each $111.55 \mathrm{Mg}$ from Table A-4, distributed over $\sim 3700$ canisters) and is best represented as Weasel Pit sludge (Willis 1995).

\subsubsection{K East and $K$ West Fuel Assemblies}

A KE fuel assembly, having $22.7 \mathrm{~kg} \mathrm{U}$ per assembiy, has a larger contribution to a Hazard Category 2 facility, than a $16.3 \mathrm{~kg} \mathrm{U} \mathrm{KW}$ fuel assembly, in spite of the fact that one element of the $\mathrm{KW}$ fuel assembly is $1.25 \%$ wt. ${ }^{235} \mathrm{U}$, opposed to the KE fuel assembly having $0.95 \%$ wt. ${ }^{235} U$. Since the $U$ was not included in Table A-1, Table A-2 provides the contribution to the Category 2 TQ for a KE fuel assembly, using $22.6 \mathrm{~kg} \mathrm{U}$ per Mark IV fuel assembly and using $0.95 \mathrm{wt} \%$ as ${ }^{235} \mathrm{U}$. Criticality does not appear a concern for KE fuel, so no criticality concern is indicated in the title of Table A-2. However, Table A-3 shows the result if there was a criticality concern. Table A- 14 provides the contribution for a $\mathrm{KW}$ 
fuel assembly, based upon a Mark IA fuel assembly having $16.3 \mathrm{~kg} U$ with no criticality concern. Since the inner element has $0.95 \mathrm{wt} \%{ }^{235} \mathrm{U}$, and the outer has $1.25 \mathrm{wt} \%{ }^{235} \mathrm{U}$, based upon the inner and outer diameters of the fuel elements, $1.15 \mathrm{wt} \%{ }^{235} \mathrm{U}$ is used to derive the ${ }^{235} \mathrm{U}$ value shown. Table A-15 provides the contribution from a $\mathrm{KW}$ fuel assembly if criticality is of concern. The differences in whether criticality is a concern or not are driven by the different values for the Category 2 TQs for ${ }^{239} \mathrm{Pu}$ and ${ }^{235} \mathrm{U}$ previously discussed.

Table 1. Comparison of K Basin Segments Of IWTP to Category 2 Threshold Quantity Values.

\begin{tabular}{|c|c|c|c|}
\hline Segment & $\begin{array}{l}\text { Maximum } \\
\text { Loading }\end{array}$ & $\begin{array}{c}\text { Ratio to Cat } 2 \text { TO with } \\
\text { Crit, Concern }\end{array}$ & $\begin{array}{l}\text { Ratio to Cat. } 2 \text { TO } \\
\text { w/out Crit. Concern }\end{array}$ \\
\hline $1 \mathrm{IXM}(\mathrm{KE} \& \mathrm{KW})$ & $1.7 \mathrm{Ci}$ TRU & $\begin{array}{l}2.2 \mathrm{E}-01 / \mathrm{IXM} \\
(2.3 \mathrm{E}-01) / \mathrm{IXM}^{\prime}\end{array}$ & $\begin{array}{l}4.3 \mathrm{E}-02 / 1 \times M \\
(4.7 \mathrm{E}-02) / 1 \times M^{\prime}\end{array}$ \\
\hline $\begin{array}{l}\text { KE Main Basin } \\
\text { Sludge }\end{array}$ & -- & $9.9 \mathrm{E}-04 / \mathrm{kg}$ sludge & $3.9 \mathrm{E}-04 / \mathrm{kg}$ sludge \\
\hline $\begin{array}{l}\text { E Weasel Pit } \\
\text { Sludge }\end{array}$ & $\cdots$ & 1. JE-02 $/ \mathrm{kg}$ sludge & $7.9 \mathrm{E}-04 / \mathrm{kg}$ sludge \\
\hline $\begin{array}{l}1 \text { KE Canister } \\
\text { Sludge }\end{array}$ & $3.15 \mathrm{~kg}$ & 3.8E-02/canister & 2.5E-03/canister \\
\hline $\begin{array}{l}1 \text { Hydrocyclone } \\
\text { (KE) }\end{array}$ & $\begin{array}{l}2 \mathrm{E}+04 \mathrm{~cm}^{3} \\
\text { sludge }\end{array}$ & $\begin{array}{l}3.0 \mathrm{E}-01 \text { (Weasel Pit) } \\
2.2 \mathrm{E}-02 \text { (main basin) }\end{array}$ & $\begin{array}{l}\text { 2.4E-02 (Weasel Pit) } \\
8.7 \mathrm{E}-03 \text { (main basin) }\end{array}$ \\
\hline 1 KE Filter Vessel & $145 \mathrm{~kg}$ & $\begin{array}{l}\text { 1.5E + OO (Weasel Pit) } \\
1.4 \mathrm{E}-01 \text { (main basin) }\end{array}$ & $\begin{array}{l}\text { 1.1E-01 (Weasel Pit) } \\
5.7 \mathrm{E}-02 \text { (main basin) }\end{array}$ \\
\hline $\begin{array}{l}\text { KE Backwash } \\
\text { Tank }\end{array}$ & $1,893 \perp(500-$ gal $)$ & $\begin{array}{l}2.9 E+00 \\
(\text { Weasel Pit sludge })^{2} \\
1.4 E+00 \\
{\text { (main basin sludge })^{2}}\end{array}$ & $\begin{array}{l}2.3 \mathrm{E}-01 \\
\text { (Weasel Pit sludge }^{2} \\
1.1 \mathrm{E}-01 \\
{\text { (main basin sludge }{ }^{2}}^{2}\end{array}$ \\
\hline $\begin{array}{l}1 \mathrm{KE} \text { Fuel } \\
\text { Assembly }\end{array}$ & -- & 7.9E-01/assembly & 4.1E-01/assembly \\
\hline $\begin{array}{l}\text { KW Main Basin } \\
\text { Sludge }\end{array}$ & -- & $9.9 \mathrm{E}-05 / \mathrm{kg}$ sludge & $3.9 E-05 / \mathrm{kg}$ sludge \\
\hline $\begin{array}{l}1 \mathrm{KW} \text { Canister } \\
\text { Sludge }\end{array}$ & $3.15 \mathrm{~kg}$ & 3.8E-02/canister & 2.5E-03/canister \\
\hline $\begin{array}{l}1 \mathrm{KW} \text { Fuel } \\
\text { Assembly }\end{array}$ & -- & 6.1E-01/assembly & 3.0E-01/assembly \\
\hline
\end{tabular}

Note:

${ }^{1}$ If $1,250 \mathrm{KW}$ canisters are processed with additional ${ }^{137} \mathrm{Cs}$ before IXM change-out.

${ }^{2}$ Practical Values, See Text 


\subsection{K EAST SYSTEM COMPONENTS (SEGMENTS)}

\subsubsection{K East Ion Exchange Modules}

The proposed IXMs are not anticipated to be significantly different from those currently used for the $\mathrm{K}$ Basins. Each IXM contains six ion exchange columns (IXC). Each IXC is 16 in. diameter Schedule 30 (0.375 in. wall thickness) pipe. Each IXM contains $0.595 \mathrm{~m}^{3}\left(21 \mathrm{ft}^{3}\right)$ of resin, which occupies approximately half the void volume of the IXM. Therefore, the void volume in each IXC is approximately 0.1 meter $\left(1 \mathrm{E}+05 \mathrm{~cm}^{3}\right)$. With fully compacted Weasel Pit sludge, $153 \mathrm{~kg}$ of sludge could theoretically be contained in each $\mathrm{XC}$. Great incentive is in place to not exceed the maximum TRU loadings to prevent the IXMs frorn being declared as TRU waste and the associated cost of disposing, compared to low level waste. Therefore, the limits shown in Table 1 are considered reasonable, yet conservative limits.

Criticality analysis to date show no concern for IXMs, due to the incentive to remove them from service prior to becoming TRU waste (Erickson 1994). Inadvertent transfer of material from one IXM to another, or back to a KE filter vessel is not believed credible, given the piping/valving arrangements and incentive to preclude TRU waste. Further discussion and derivations are discussed in Attachment $A$.

\subsubsection{Hydroc:yclones}

The four, physically separated, hydrocyclones are used only in KE, and are estimated, based upon Vendor provided data, to have an internal volume of $2 E+04 \mathrm{~cm}^{3}$ each. One hydrocyclone is for fuel retrieval and the other three are for sludge retrieval. For fuel retrieval, the flow from the primary cleaning station and damaged canister station will flow to the single hydrocyclone. Two values (for a hydrocyclone) are shown in the third and fourth columns of Table 1, depending if it contains Weasel Pit or main basin sludge. It should be noted that the density of the Weasel Pit sludge is estimated as $1.535 \mathrm{~g} / \mathrm{cm}^{3}$ (Table A-4), while main basin sludge is estimated as $1.13 \mathrm{~g} / \mathrm{cm}^{3}$. The hydrocyclones are not physically capable of containing Cat. 2 TQs of Weasel Pit or main basin sludge. The overflow containing an estimated $23.5 \%$ (conservatively rounded up to $30 \%$ ) of the siludge/solids in the hydrocyclones will discharge to the overflowweir located in the west section of the Weasel Pit and the remaining $70 \%$ of the sludge/solids contained in the underflow will discharge to the east section of the Weasel Pit.

\subsubsection{K East Filter Vessels}

Each of the four KE filter vessels are physically separated, contain a pleated filter element, and have an internal free volume of approximately $9.46 \mathrm{E}+04$ centimeter $^{3}(\mathrm{~cm})$ (25 gallon [gal]). The pleated filter area is $71,500 \mathrm{~cm}^{2}\left(77 \mathrm{ft}^{2}\right)$. The available void volume within the filter cell of $9.46 \mathrm{E}+04 \mathrm{~cm}^{3}$ with a Weasel Pit sludge density of $1.535 \mathrm{~g} / \mathrm{cm}^{3}$, could contain an absolute maximum of $145 \mathrm{~kg}$ of sludge. It is extremely unlikely that a filter element would ever contain $145 \mathrm{~kg}$ of sludge, as at a certain-set point of differential pressure, the sludge will be discharged to the backwash tank prior to the filter ever reaching its full volumetric capacity. A very conservative estimate is that about $0.32-\mathrm{cm}$ 
(1/8-in) thickness of sludge will accumulate on the filter element before being discharged to the backwash tank. A $0.32-\mathrm{cm}$ depth of sludge over the entire $71,500 \mathrm{~cm}^{2}$ filter area represents $2.29 \mathrm{E}+04 \mathrm{~cm}^{3}$, or $35.1 \mathrm{~kg}$ of Weasel Pit sludge, which equates to 0.028 of a TO 2 value. Each time a filter cell is backwashed to the backwash tank, $227 \mathrm{~L}(60 \mathrm{gal})$ of water will be used. Existing filters for the $K$ Basins have been studied extensively from a criticality standpoint and the results show no criticality concern (Schwinkendorf 1994). It is not anticipated that the new filter vessels for KE will be treated any different, so far as limitations are based on criticality concerns.

\subsubsection{K East Filter Backwash Tank}

The KE filter backwash tank has a $1,893 \mathrm{~L}\left(1.89 E+06 \mathrm{~cm}^{3}\right)$ (500 gal) capacity. It is not envisioned that the backwash tank would ever contain large quantities of sludge, as at a pre-set liquid level in the tank (nominally $80 \%$ to $90 \%$ capacity), the backwash tank contents will be pumped to the east section of the Weasel Pit. Since each filter cell discharge will contain a maximum of $2.29 \mathrm{E}+04 \mathrm{~cm}^{3}$ (6 gal) of sludge and will be backwashed with a minimum of $2.27 \mathrm{E}+05 \mathrm{~cm}^{3}$ (60 gal) of water, $-10 \%$ of the backwash tank volume would contain sludge with the remaining volume occupied with water. Therefore, the backwash tank could practically hold only 0.23 of a TQ 2 value of Weasel Pit sludge for the situation that criticality concerns are not warranted.

\subsubsection{K East Submerged Filters}

There are currently two existing submerged cartridge units that can be operated either independently or together in parallel configuration. These have been valved out of service for the KE IWTP, but can be brought back on line if needed.

\subsection{K WEST INTEGRATED WATER TREATMENT PROJECT}

\subsection{PROCESS DESCRIPTION}

The KW IWTP will consist mainly of the two existing recirculation pumps and three IXMs. For the Fuel Retrieval Sub-Project, flow from the de-lidding station and primary cleaning station (washing machine) will be transferred to the IXMs. The flow from the downdraft table station and the damaged canister station will be pumped via the existing recirculation pumps. From the recirculation pumps, at least $70 \%$ of the flow will be returned to the basin with the remaining $30 \%$ discharged to the IXMs. The existing submerged filters which are downstream of the pumps, are currently valved out of service, but can be brought on line, if desired. The flow from all three IXMs is discharged back to the basin.

During Debris Removal, the flow from the debris removal station will be discharged to the main recirculation pump suction header. The same routing is then provided as for the damaged canister station and downdraft table. 
Process flow diagrams for $\mathrm{KW}$ are included in Appendix A.

\subsection{K WEST MATERIAL AT RISK}

\subsubsection{K West Main Basin Sludge}

The $\mathrm{KW}$ main basin sludge is rather benign as far as isotopic loading, due to the $\mathrm{KW}$ canisters being sealed. The $\mathrm{KW}$ basin floor sludge is expected to contain environmental pollutants such as dust, dirt, paint chips, etc. (Willis 1995). Nonetheless, KW main basin sludge is conservatively taken to be $1 / 10$ the quantity and radionuclide concentration of KE main basin sludge.

\subsubsection{K West Canister Sludge}

The KW canister sludge has not been estimated, but should be bounded in both volume and radionuclide concentration by KE canister sludge, due to the corrosion inhibiter placed into the sealed canisters.

\subsubsection{K West Fuel Assemblies}

The KW fuel assemblies are described in Section 2.2.3 above. The KW fuel assemblies have been stored in sealed canisters containing a corrosion inhibitor (potassium nitrate). Additionally, there are fourteen 26 in. length MK1 A assemblies also stored in $\mathrm{KW}$.

\subsection{K WEST SYSTEM COMPONENTS (SEGMENTS)}

\subsubsection{K West lon Exchange Modules}

The three IXMs in KW are similar to those in KE. During de-lidding operations for fuel retrieval in $\mathrm{KW}$, it is estimated that each canister barrel contains $0.143 \mathrm{Ci}$ of ${ }^{137} \mathrm{Cs}$ (WHC 1996b), which will be pumped to the three IXMs, in parallel configuration. For 1,250 canisters (6 months of operation), this represents $358 \mathrm{Ci}$ of ${ }^{137} \mathrm{Cs}$. This additional loading is shown in parenthesis in Table 1-1 for the IXMs. This is very conservative, as the three IXMs are operated in parallel configuration. Further discussion and derivations are discussed in Attachment $\mathrm{A}$.

\subsubsection{K West Submerged Filters}

There are currently two existing submerged cartridge units that can be operated either independently or in parallel configuration. It is anticipated these will be valved out of service for the KW IWTP, but can be refitted and brought back on line if needed. 
WHC-SD-SNF-RPT-012, Rev. 0

\subsection{K EAST BOUNDING SCENARIOS FOR HAZARD CATEGORIZATION}

Based upon the above, which shows that criticality concern for KE is not warranted, the bounding scenarios for Hazard Category purposes are assessed. In the first scenario, it is assumed a fitter vessel has a very conservative $0.32 \mathrm{~cm}(1 / 8 \mathrm{in}$. $)$ thickness of canister sludge (modeled as Weasel Pit sludge) just before backwashing to the backwash tank. This represents $35.1 \mathrm{~kg}$ of canister sludge, or 0.028 of a Category $2 \mathrm{TQ}$. This value can only occur if sludge retrieval is not taking place at the same time, as main basin sludge has approximately $49 \%$ the isotopic concentration as canister sludge. During fuel retrieval, the equivalent of one fuel assembly of the 14 in the "washing machine" is postulated to disintegrate. No credit for any fuel pieces greater than $1 / 4$ in. that would be filtered by the fuel retrieval filter is taken. The entire fuel assembly is assumed to be directed to the hydrocyclone. The hydrocyclone directs at least $70 \%$ to the east section of the Weasel Pit, with the remaining $30 \%$ going to the west section of the Weasel Pit. It is conservatively assumed that all the overflow flows over the weir on the west end of the west section of the Weasel Pit and then flows to the filter vessel. The total flow from $30 \%$ of the disintegrated fuel assembly, which represents 0.123 of a TO 2 value, then is added to that assumed in the filter vessel to already exist, or $0.123+0.028=0.151$ of a TQ 2 value. This is still conservative, as the fuel assembly would most likely be distributed fairly evenly over all four filter vessels. With two fuel assemblies disintegrating in the "washing machine", the equivalent of $0.123+0.123+0.028=0.274$ of a TQ 2 value would result. In fact, it would take more than seven fuel assemblies disintegrating with all residing in a single filter vessel before the result would be over a TO 2 value.

A second scenario again has one of the filter vessels loaded up already with 0.028 of a TQ 2 value conly possible if sludge retrieval operations are not also taking place, as noted above), but this time one fuel assembly disintegrates (or equivalent of one assembly) while being tipped onto the downdraft table. This is a much less credible scenario, as the "washing machine" is the location where the fuel assemblies will first encounter any significant mechanical action (rolling of the assemblies about each other horizontally). In this second scenario, the most likely time for a fuel assembly to then disintegrate is when tipped from the tipping station onto the downdraft table for further cleaning and inspection. For this case, if the equivalent of one fuel assembly disintegrates, taking no credit for the $1 / 4$-in screens, 0.41 of a TQ 2 value from the KE fuel assembly would flow to the filter vessels. Even if the entire assembly went to only one of the four filter vessels, that vessel would contain $0.028+0.41=0.438$ of a TO 2 value in any single filter vessel. If two were to disintegrate, the value would be 0.85 in the filter vessel. However, the more likely situation would be for the fuel assemblies to be fairly evenly distributed into the four filter vessels, providing about $(0.028)+(0.41 \div 4)+(0.41 \div 4)=0.233$ of a TQ 2 value.

A third scenario is one in which the backwash tank resides with a nominal conservative loading of sludge from recent backflushes from the four filter vessels, or $4 \times 0.028=0.112$ of a TO 2 value. These four backflushes also would have provided $910 \mathrm{~L}$ (240 gal) to the backwash tank. It is further assumed that due to some electrical or mechanical problem, the backwash tank pumping system becomes inoperative. It is then assumed that one fuel assembly disintegrates when tipped onto the downdraft table. The fuel assembly at 0.41 of a TO 2 value then flows to the four filter vessels, each now assumed to contain a conservative loading of $0.32 \mathrm{~cm}(1 / \mathrm{i}$ in.) of sludge or 0.028 of a TO 2 value since the last backflush. The differential pressure switch then activates and the 
entire ensemble, consisting of one fuel assembly and contents of four filter vessels is then automatically discharged sequentially to the backwash tank. With an initial loading of 0.112 in the backwash tank, plus the fuel assembly at 0.41 , plus the four filter vessels at 0.028 each, a total of $0.112+0.41+(4)(0.028)=0.634$ of a TO 2 value resides in the backwash tank. At this point, $1,820 \mathrm{~L}(480 \mathrm{gal})$ of water also resides in the backflush tank. The total volume now occupied within the backwash tank is $1,820 \mathrm{~L}$ ( $480 \mathrm{gal}$ ) of water plus 8 "slugs" of sludge $(8 \times 22.9 \mathrm{~L}$ per "slug" $=183 \mathrm{~L})$. This total is $2,000 \mathrm{~L}$, which exceeds the volume of the backwash tank. If sludge retrieval is taking place at the same time, the assumed value for the sludge (assumed to be canister or Weasel Pit sludge) would have an even lower value, as the main basin sludge has only $49 \%$ the contribution per $\mathrm{kg}$ of canister sludge. If two fuel assemblies were to disintegrate at the same time while being tipped onto the downdraft table, $0.112+.41+0.41+(4)(0.028)=1.04$ of a TQ 2 value would exist in the backwash tank, yet the backwash tank cannot hold this total volume. Such an event (two fuel assemblies totally disintegrating, taking no credit for

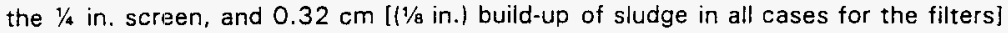
occurring under the conditions so postulated for the filter vessels and backwash tank is considered to be incredible.

\subsection{K EAST HAZARD CATEGORIZATION CONCLUSION}

Based upon all three scenarios presented, and since criticality is precluded, it is deemed that the IWTP for KE has a Preliminary Hazard Category of 3.

\subsection{KW BOUNDING SCENARIOS FOR HAZARD CATEGORIZATION}

$\mathrm{K}$ West does not have hydrocyclones or backwash filter vessels, but only IXMs, and possibly the two submerged filters (if retained). The fuel is slightly more reactive, but due to the lower uranium content $(16.3 \mathrm{~kg})$, one $\mathrm{KW}$ fuel assembly (Mark IA), with or without criticality concerns, has TO 2 ratios (Tables A-14 and A-15) less than KE fuel (Tables A-2 and $\mathrm{A}-3$ ). The first scenario for the KW Hazard Categorization, is a fuel assembly disintegrating in the $\mathrm{KW}$ "washing machine". It is anticipated that the physical integrity of the KW fuel is better than KE fuel due to the fuel assemblies being in sealed canisters containing a corrosion inhibiter. Up to three IXMs can be operated together in parallel configuration and are assumed to have their nominal maximum loading, shown in Table A-13 (with criticality concern and each containing $358 \mathrm{Ci}$ of ${ }^{137} \mathrm{Cs}$ ), of 0.23 just before the fuel assembly disintegrates in the KW "washing machine" at the primary cleaning station. It is likely that each of the six internal ion exchange columns in each of the three IXMs are fairly evenly loaded, which equates to $\sim 0.038$ of a TO 2 value with criticality concerns, although no credit is taken for this distribution. For criticality purposes, a wall thickness of $0.375 \mathrm{in}$. is considerably more than the $0.0625 \mathrm{in}$. wall thickness of a $55 \mathrm{gal}$ drum where criticality evaluations routinely take credit for physical separation. With criticality concern, the fuel assembly would have 0.61 of a TQ 2 value (Table A-15). Therefore the total resulting in the IXM (if it could hold it) if the entire fuel assembly went to only one IXM, would be $0.61+0.23=0.84$ of a TQ 2 value. This is very conservative, as there are usually three IXMs operating in parallel. A more realistic value would be $0.23+.61 / 3=$ 
0.43 of a TC. 2 value in each IXM. For two fuel assemblies disintegrating, the most realistic result would be $0.23+.61 / 3+.61 / 3=0.64$ of a TQ 2 value. For three, the likely result would be each IXM having 0.84 of a TO 2 value, and for four, the result would be 1.04 of a TQ 2 value. Considering the physical state of the KW fuel assemblies, more than three fuel assemblies disintegrating out of a total of 14 in the "washing machine" is estimated to be incredible.

A second scenario is in which the fuel assemblies are being tipped onto the do.vndraft table and a fuel element disintegrates at this time. The flow from the downdraft table is pumped through the existing recirculation pumps with about $70 \%$ being returned to the basin and the remaining $30 \%$ transferred to the IXMs. With each IXM already containing 0.23 of a TQ 2 value, the dissintegrating fuel assembly represents 0.61 of a TQ 2 value, resulting in 0.84 in the IXM if only one IXM was to receive the fuel assembly. A more realistic result is with each of the three IXMs receiving about $1 / 3$ of the disintegrated fuel assembly. This provides $0.23+.61 / 3=0.43$ of a TO 2 value in any IXM and takes no credit for only $30 \%$ of the flow from the recirculating pumps going to the IXMs. Two disintegrating fuel assemblies would realistically represent $0.23+0.61 / 3+0.61 / 3=$ 0.64 of a TQ 2 value without taking credit for the portion that would go to the basin instead. Three fuel assemblies, would represent 0.84 of a TO 2 value in the IXM, again taking no credit for the portion that would actually return to the basin. It would take more than three fuel assemblies, with criticality concern, to exceed the TQ 2 value in the IXMs. Taking credit for only $30 \%$ of the flow going to the IXMs from the recirculating pumps, it would take more than 11 fuel assemblies disintegrating before a single IXM would exceed a TQ 2 value with the normal situation of three IXMs operating in parallel.

\subsection{K WEST HAZARD CATEGORIZATION CONCLUSION}

Based upon the above scenarios, since even if criticality concerns are initially assumed, it would require more than three $\mathrm{KW}$ fuel assemblies to disintegrate on the downdraft table or at the primary cleaning station to exceed the TO 2 value. Given the conservatisms assumed, the IWTP for $\mathrm{KW}$ is deemed to have a Preliminary Hazard Category of 3 .

\subsection{INSTALLATION RISKS}

Maximization of existing $\mathrm{KE}$ and $\mathrm{KW}$ equipment is planned to be utilized. The instaliation of the required new structures, systems, and components of the IWTP poses no significant undesirable effects compared to the current KE and KW Basin operations. The installation of such equipment does not change the Preliminary Hazard Category 3 determination for the IWTP. Any potential structural loads will be managed in a manner, including if necessary, the movement of fuel canisters, to ensure installation does not affect the existing stored fuel and existing basin equipment. 


\subsection{REFERENCES}

DOE, 1992a, Hazard Categorization and Accident Analysis Techniques for Compliance with DOE Order 5480.23, Nuclear Safety Analysis Reports, DOE-STD-1027-92, U.S. Department of Energy, Washington, D.C.

DOE, 1992b, Nuclear Safety Analysis Reports, DOE Order 5480.23, U.S. Department of Energy, Washington, D.C.

Erickson, 1994, Criticality Safety Evaluation of the 100K Area lon Exchange Modules and Ion Exchange Columns, WHC-SD-NR-CSER-011, Rev. 1, Westinghouse Hanford Company, Richland, Washington.

LATA, 1996, Integrated Water Treatment Project - Topical Report - Hazard Categorization In Support of The Preliminary Design, June 25, 1996, Los Alamos Technical Associates, Richland, Washington.

Neeley, V.I. and H.E. Handler, Measurement of Multiplication Constant For Slightly Enriched Homogeneous $\mathrm{UO}_{3}$-Water Mixtures and Minimum Enrichment For Criticality, HW-70310, Hanford Atomic Products Operation, Richland, Washington.

Schwinkendorf, 1994, Criticality Safety Evaluation Report for K Basin Filter Cartridges, WHC-SD-SNF-CSER-002, Rev. O, Westinghouse Hanford Company, Richland, Washington.

Willis, 1995, 105-K Basin Material Design Basis Feed Description For Spent Nuclear Fuel Project Facilities, WHC-SD-SNF-TI-009, Westinghouse Hanford Company, Richland, Washington.

Wittekind, 1992, K Basin Criticality Evaluation for Irradiated Fuel Canisters in Sludge, WHC-SD-NR-CSER-001, Rev. O, Westinghouse Hanford Company, Richland, Washington.

WHC, 1993, MCNP Calculations of K-infinity for 10OKE Basin Sludge Samples, WHC-SDNR-CSER-008, Rev. 1, Westinghouse Hanford Company, Richland, Washington.

WHC, 1995, K Basins Process Standards, C-304, Draft, Westinghouse Hanford Company, Richland, Washington.

WHC, 1996a, Statement of Work For Preliminary Design of the K Basin Integrated Water Treatment Systems Project, WHC-SD-SOW-007, Rev. O, Westinghouse Hanford Company, Richland, Washington.

WHC, 1996b, Data Analysis of Gas and Liquid from $K$ West Basin Fuel Canisters; 1995 Samples, WHC-SD-SNF-ANAL-008, Westinghouse Hanford Company, Richland, Washington. 
WHC-SD-SNF-RPT-012, Rev. O

\section{APPENDIX B}

ATTACHMENT A

\section{RADIOLOGICAL INVENTORIES FOR 105-K BASINS}


WHC-SD-SNF-RPT-012, Rev. 0

This page intentionally blank.

B-Attach-A-2 
WHC-SD-SNF-RPT-012, Rev. 0

RADIOLOGICAL INVENTORIES FOR 105-K BASINS

The most recent summary of radiological inventories for the $105-\mathrm{K}$ Basins is described in 105-K Basin Material Design Basis Feed Description for Spent Nuclear Fuel Project Facilities, WHC-SD-SNF-TI-009 (Willis 1995). That document (Willis 1995) as well Spent Nuclear Fuel Project Technical Data Book, WHC-SD-SNF-TI-015 (WHC 1995) are the sources for the inventaries used.

\section{A.1 INVENTORIES}

\section{A.1.1 K East Fuel}

There are 3,666 canisters in KE containing 50,683 fuel assemblies. The total fuel mass in the basin is 1,238 metric tons. Of the total mass, there are $1,143,600 \mathrm{~kg}$ of uranium and $2,155 \mathrm{~kg}$ of plutonium. There are approximately $2.5 \mathrm{E}+\mathrm{O} 7$ curies (Ci) of fissior and activation products, and approximately $4.3 \mathrm{E}+06 \mathrm{Ci}$ of actinides (U and TRU isotop :s) associated with the fuel. Willis (1995) provides information on the fuel assemblies' characteristics. The average MK IV fuel element has a uranium weight of $22.7 \mathrm{~kg}$. Willis (1995) provides recommended fuel loading summaries depending on whether the intended purpose is for safety/regulatory assessment, heat generation estimate, or shielding purpose. The safety/regulatory values were selected by (Willis 1995 ) by identifying the isotopic mixture of components expected to yield the largest radiological dose to people per unit of material released. The selection process used to compare the isotopic mixtures used a reduced set of radionuclides which are anticipated to dominate dose calculations for accident purposes as shown in Table A-1. For Hazard Categorization purposes, the quantities of ${ }^{235} \mathrm{U}$ and ${ }^{238} \mathrm{U}$ in the uranium metal are also considered. Table A-2 includes the uranium and provides the contribution to a Hazard Category 2 facility/process without criticality concern, while Table A-3 provides similar information but if criticality is a concern.

Table A-1. Safety/Regulatory Assessment Design Basis Summary (Based on MK IV Fuel at $16.72 \%{ }^{240} \mathrm{Pu}$, Aged 16 Yrs).

\begin{tabular}{|l|c|l|c|}
\hline \multicolumn{1}{|c|}{ Isotope } & Activity (Ci/MTU') & \multicolumn{1}{|c|}{ Isotope } & Activity (Ci/MTU') \\
\hline${ }^{90} \mathrm{Sr}$ & $7.84 \mathrm{E}+03$ & ${ }^{240} \mathrm{Pu}$ & $1.28 \mathrm{E}+02$ \\
\hline${ }^{137} \mathrm{Cs}$ & $1.08 \mathrm{E}+04$ & ${ }^{241} \mathrm{Pu}$ & $9.25 \mathrm{E}+03$ \\
\hline${ }^{238} \mathrm{Pu}$ & $1.71 \mathrm{E}+02$ & ${ }^{242} \mathrm{Pu}$ & $1.05 \mathrm{E}-01$ \\
\hline${ }^{239} \mathrm{Pu}$ & $1.58 \mathrm{E}+02$ & ${ }^{241} \mathrm{Am}$ & $3.58 \mathrm{E}+02$ \\
\hline
\end{tabular}

Note:

'MTU = Metric Tons Uranium 
Table A-2. Isotopic Content of One Average Fuel Assembly (KE, No Criticality Concern).

\begin{tabular}{|l|c|c|c|}
\hline \multicolumn{1}{|c|}{ Isotope } & Ci per Assembly & $\begin{array}{c}\text { Category 2 TO } \\
\text { (Ci) }\end{array}$ & $\begin{array}{c}\text { Ratio of Total } \\
\text { Ci/Cat. 2 TO }\end{array}$ \\
\hline${ }^{90} \mathrm{Sr}$ & $1.78 \mathrm{E}+02$ & $2.2 \mathrm{E}+04$ & $8.1 \mathrm{E}-03$ \\
\hline${ }^{137} \mathrm{Cs}$ & $2.45 \mathrm{E}+02$ & $8.9 \mathrm{E}+04$ & $2.8 \mathrm{E}-03$ \\
\hline${ }^{235} \mathrm{U}$ & $4.64 \mathrm{E}-04$ & $2.4 \mathrm{E}+02$ & $1.9 \mathrm{E}-06$ \\
\hline${ }^{238} \mathrm{U}$ & $7.55 \mathrm{E}-03$ & $2.4 \mathrm{E}+02$ & $3.1 \mathrm{E}-05$ \\
\hline${ }^{238} \mathrm{Pu}$ & $3.88 \mathrm{E}+00$ & $6.2 \mathrm{E}+01$ & $6.3 \mathrm{E}-02$ \\
\hline${ }^{239} \mathrm{Pu}$ & $3.59 \mathrm{E}+00$ & $5.6 \mathrm{E}+01$ & $6.4 \mathrm{E}-02$ \\
\hline${ }^{240} \mathrm{Pu}$ & $2.91 \mathrm{E}+00$ & $5.5 \mathrm{E}+01$ & $5.3 \mathrm{E}-02$ \\
\hline${ }^{241} \mathrm{Pu}$ & $2.10 \mathrm{E}+02$ & $2.9 \mathrm{E}+03$ & $7.2 \mathrm{E}-02$ \\
\hline${ }^{242} \mathrm{Pu}$ & $2.38 \mathrm{E}-03$ & $2.8 \mathrm{E}+01$ & $8.5 \mathrm{E}-05$ \\
\hline${ }^{241} \mathrm{Am}$ & $8.13 \mathrm{E}+00$ & $5.5 \mathrm{E}+01$ & $1.5 \mathrm{E}-01$ \\
\hline & & Sum of Ratios & $4.1 \mathrm{E}-01$ \\
\cline { 3 - 4 } & & &
\end{tabular}

Table A-3. Isotopic Content of One Average Fuel Assembly (KE, With Criticality Concern).

\begin{tabular}{|l|c|c|c|}
\hline \multicolumn{1}{|l|}{ Isotopie } & Giper Assembly & $\begin{array}{c}\text { Category 2 TO } \\
\text { [Ci] }\end{array}$ & $\begin{array}{c}\text { Ratio of Total } \\
\text { Ci/Cat, 2 TQ }\end{array}$ \\
\hline${ }^{90} \mathrm{Sr}$ & $1.78 \mathrm{E}+02$ & $2.2 \mathrm{E}+04$ & $8.1 \mathrm{E}-03$ \\
\hline${ }^{137} \mathrm{Cs}$ & $2.45 \mathrm{E}+02$ & $8.9 \mathrm{E}+04$ & $2.8 \mathrm{E}-03$ \\
\hline${ }^{235} \mathrm{U}$ & $4.64 \mathrm{E}-04$ & $1.51 \mathrm{E}-03^{1}$ & $3.1 \mathrm{E}-01$ \\
\hline${ }^{238} \mathrm{U}$ & $7.55 \mathrm{E}-03$ & $2.4 \mathrm{E}+02$ & $3.1 \mathrm{E}-05$ \\
\hline${ }^{238} \mathrm{Pu}$ & $3.88 \mathrm{E}+00$ & $6.2 \mathrm{E}+01$ & $6.3 \mathrm{E}-02$ \\
\hline${ }^{239} \mathrm{Pu}$ & $3.59 \mathrm{E}+00$ & $2.8 \mathrm{E}+01^{2}$ & $1.3 \mathrm{E}-01$ \\
\hline${ }^{240} \mathrm{Pu}$ & $2.91 \mathrm{E}+00$ & $5.5 \mathrm{E}+01$ & $5.3 \mathrm{E}-02$ \\
\hline${ }^{241} \mathrm{Pu}$ & $2.10 \mathrm{E}+02$ & $2.9 \mathrm{E}+03$ & $7.2 \mathrm{E}-02$ \\
\hline${ }^{242} \mathrm{Pu}$ & $2.38 \mathrm{E}-03$ & $2.8 \mathrm{E}+01$ & $8.5 \mathrm{E}-05$ \\
\hline${ }^{241} \mathrm{Am}$ & $8.13 \mathrm{E}+00$ & $5.5 \mathrm{E}+01$ & $1.5 \mathrm{E}-01$ \\
\hline
\end{tabular}

'Based on $700 \mathrm{~g}$ for ${ }^{235} \mathrm{U}$ for TQ value

${ }^{2}$ Based on $450 \mathrm{~g}$ for ${ }^{239} \mathrm{Pu}$ for TQ value 


\section{A.1.2 K East Sludge}

There is estimated to be approximately $51 \mathrm{~m}^{3}$ of sludge in KE distributed as shown in Table A-4. Table A-5 shows the major radionuclide constituents of the sludge. Table A6 shows the contribution per $\mathrm{kg}$ of KE sludge to a TQ ratio of 1.0 for a Hazard Category 2 facility/process, assuming no criticality concern, while Table A-7 provides the result if criticality is a concern.

Table A-4. Physical Properties of 105-K East Basin Sludge.'

\begin{tabular}{|c|c|c|c|c|c|}
\hline Basin Location & $\begin{array}{l}\text { Volume } \\
\left(\mathrm{m}^{3}\right)\end{array}$ & $\begin{array}{l}\text { Mass } \\
(\mathrm{Mg})\end{array}$ & $\begin{array}{l}\text { Density } \\
\text { (g/mi) }\end{array}$ & $\begin{array}{c}\text { Particle size } \\
\text { (um) }\end{array}$ & $\begin{array}{c}\text { Solids }^{3} \\
\text { (weight } \% \text { ) }\end{array}$ \\
\hline Weasel Pit & 11.86 & 18.20 & 1.535 & 1.53 & 51.29 \\
\hline Tech View Pit & 0.43 & 0.66 & 1.535 & 1.53 & 51.29 \\
\hline Wash Pit & 1.37 & 2.11 & 1.535 & 1.53 & 51.29 \\
\hline Sandfilter Backwash Pit & 6.27 & 8.25 & 1.31 & 1.57 & 32.00 \\
\hline South Load-out Pit & 1.09 & 1.67 & 1.535 & 1.53 & 51.29 \\
\hline Fuel Canisters & 7.84 & 11.55 & $1.13-1.535$ & $1.53-1.68$ & $17.52-51.29$ \\
\hline Basin Floor & 21.65 & 24.46 & 1.13 & 1.68 & 17.52 \\
\hline Total & 50.51 & 66.90 & & & \\
\hline
\end{tabular}

Notes:

'Willis (1995)

${ }^{2}$ Particle size is average size of all particles in sample. Most particles are $<2 \mu \mathrm{m}$ diameter; over half of volume is $>20-40 \mu \mathrm{m}$.

${ }^{3}$ Weight percent of solids is the fractions of solids in the sludge (the remainder is water).

Table A-5. Average Radionuclide Composition of K East Basin Sludge $(\mu \mathrm{Ci} / 9){ }^{1}$

\begin{tabular}{|l|c|c|c|}
\hline \multicolumn{1}{r|}{ Isotope } & Weasel Pit & Main Basin & Sandfilter Backwash Pit \\
\hline${ }^{3} \mathrm{H}$ & $1.27 \mathrm{E}-02$ & $4.23 \mathrm{E}-03$ & $7.81 \mathrm{E}-02$ \\
\hline${ }^{60} \mathrm{Co}$ & $1.03 \mathrm{E}+01$ & $2.97 \mathrm{E}+00$ & $4.12 \mathrm{E}+00$ \\
\hline${ }^{90} \mathrm{Sr}$ & $4.48 \mathrm{E}+01$ & $4.47 \mathrm{E}+01$ & $2.19 \mathrm{E}+01$ \\
\hline${ }^{137} \mathrm{Cs}$ & $8.56 \mathrm{E}+01$ & $4.62 \mathrm{E}+01$ & $2.82 \mathrm{E}+01$ \\
\hline${ }^{154} \mathrm{Eu}$ & $9.51 \mathrm{E}+00$ & $4.39 \mathrm{E}+00$ & $4.05 \mathrm{E}+00$ \\
\hline${ }^{155} \mathrm{Eu}$ & $8.78 \mathrm{E}+00$ & $2.95 \mathrm{E}+00$ & $3.18 \mathrm{E}+00$ \\
\hline${ }^{234} \mathrm{U}$ & $1.37 \mathrm{E}-01$ & $2.34 \mathrm{E}-03$ & \\
\hline${ }^{235} \mathrm{U}$ & $1.31 \mathrm{E}-02$ & $4.93 \mathrm{E}-04$ & \\
\hline${ }^{238} \mathrm{Pu}$ & $8.11 \mathrm{E}+00$ & $2.51 \mathrm{E}+00$ & $8.01 \mathrm{E}+00$ \\
\hline${ }^{239} \mathrm{Pu},{ }^{240} \mathrm{Pu}$ & $2.90 \mathrm{E}+01$ & $1.48 \mathrm{E}+01$ & $2.85 \mathrm{E}+01$ \\
\hline${ }^{241} \mathrm{Pu}$ & $5.90 \mathrm{E}+01$ & $2.76 \mathrm{E}+01$ & $1.94 \mathrm{E}+01$ \\
\hline${ }^{241} \mathrm{Am}{ }^{2}$ & $6.48 \mathrm{E}+00$ & $4.17 \mathrm{E}+00$ & $1.12 \mathrm{E}+01$ \\
\hline
\end{tabular}

Notes:

WHC 1995

${ }^{2}$ Based on GEA (Gamma Energy Analysis) 
Table A-6. K East Main Basin Sludge Contribution to Hazard Category 2 Threshold Quantities Per Kilogram Sludge (No Criticality Concern).

\begin{tabular}{|c|c|c|c|c|}
\hline Isotope & $\mu \mathrm{Cl} / \mathrm{g}$ & CliKg Sludge & $\begin{array}{c}\text { Category } 2 \\
\text { TO Ci }\end{array}$ & $\begin{array}{l}\text { Ratio of Total } \\
\text { Ci/Cat. } 2 \text { TO }\end{array}$ \\
\hline${ }^{3} \mathrm{H}$ & $4.23 E-03$ & 4.23E-06 & $3.0 E+05$ & $1.4 \mathrm{E}-11$ \\
\hline${ }^{60} \mathrm{Co}$ & $2.97 E+00$ & 2.97E-03 & $1.9 E+05$ & 1.6E-08 \\
\hline${ }^{90} \mathrm{Sr}$ & $4.47 E+07$ & 4.47E-02 & $2.2 E+04$ & 2.0E-06 \\
\hline${ }^{137} \mathrm{Cs}$ & $4.62 E+01$ & 4.62E-02 & $8.9 E+04$ & $5.2 \mathrm{E}-07$ \\
\hline${ }^{154} \mathrm{Eu}$ & $4.39 E+00$ & $4.39 \mathrm{E}-03$ & $1.1 E+05$ & $4.0 \mathrm{E}-08$ \\
\hline${ }^{155} \mathrm{Eu}$ & $2.95 E+00$ & $2.95 \mathrm{E}-03$ & $7.3 E+05$ & 4.0E-09 \\
\hline${ }^{234} \mathrm{U}$ & $2.34 E-03$ & $2.34 \mathrm{E}-06$ & $2.2 E+02$ & 1.1E-08 \\
\hline $235 \mathrm{U}$ & $4.93 \mathrm{E}-04$ & $4.93 \mathrm{E}-07$ & $2.4 \mathrm{E}+02$ & 2.1E-09 \\
\hline${ }^{238} \mathrm{Pu}$ & $2.51 E+00$ & $2.51 \mathrm{E}-03$ & $6.2 E+01$ & 4.0E-05 \\
\hline${ }^{239 / 240} \mathrm{Pu}$ & $1.48 \mathrm{E}+01$ & 1.48E-02 & $5.6 \mathrm{E}+01$ & $2.6 \mathrm{E}-04$ \\
\hline${ }^{241} \mathrm{Pu}$ & $2.76 \mathrm{E}+01$ & $2.76 \mathrm{E}-02$ & $2.9 E+03$ & $9.5 \mathrm{E}-06$ \\
\hline${ }^{247} \mathrm{Am}^{\mathrm{C}}$ & $4.17 E+00$ & 4.17E-03 & $5.5 \mathrm{E}+01$ & 7.6E-05 \\
\hline & & & Sum of Ratios & 3.9E-04 \\
\hline
\end{tabular}

Table A-7. K East Main Basin Sludge Contribution to Hazard Category 2 Threshold Quantities Per Kilogram Sludge (With Criticality Concern).

\begin{tabular}{|c|c|c|c|c|}
\hline Isotope & $\mu \mathrm{Cl} / \mathrm{g}$ & Ci/Kg Sludge & $\begin{array}{c}\text { Category } 2 \text { TO } \\
\text { (Ci) }\end{array}$ & $\begin{array}{l}\text { Ratio of Total } \\
\text { Cil Cat } 2 \text { TO }\end{array}$ \\
\hline${ }^{3} \mathrm{H}$ & $4.23 E-03$ & 4.23E-06 & $3.0 E+05$ & $1.4 \mathrm{E}-11$ \\
\hline${ }^{60} \mathrm{Co}$ & $2.97 E+00$ & $2.97 \mathrm{E}-03$ & $1.9 E+05$ & $1.6 \mathrm{E}-08$ \\
\hline${ }^{90} \mathrm{Sr}$ & $4.47 E+01$ & 4.47E-O2 & $2.2 E+04$ & 2.OE-O6 \\
\hline${ }^{137} \mathrm{Cs}$ & $4.62 E+01$ & $4.62 \mathrm{E}-02$ & $8.9 E+04$ & $5.2 \mathrm{E}-07$ \\
\hline${ }^{154} \mathrm{Eu}$ & $4.39 E+00$ & $4.39 \mathrm{E}-03$ & $1.1 \mathrm{E}+05$ & 4.OE-O8 \\
\hline${ }^{155} \mathrm{Eu}$ & $2.95 E+00$ & $2.95 \mathrm{E}-03$ & $7.3 E+05$ & 4.OE-O9 \\
\hline${ }^{234} \mathrm{U}$ & 2.34E-03 & $2.34 \mathrm{E}-06$ & $2.2 \mathrm{E}+02$ & 1.1E-08 \\
\hline $235 U$ & $4.93 E-04$ & 4.93E-07 & $1.5 \mathrm{E}-03^{1}$ & 3. $3 E-04$ \\
\hline${ }^{238} \mathrm{Pu}$ & $2.51 E+00$ & $2.51 \mathrm{E}-03$ & $6.2 \mathrm{E}+01$ & 4.0E-05 \\
\hline${ }^{239 / 240} \mathrm{Pu}$ & $1.48 E+01$ & $1.48 \mathrm{E}-\mathrm{O} 2$ & $2.8 \mathrm{E}+01^{2}$ & $5.3 \mathrm{E}-04$ \\
\hline${ }^{241} \mathrm{Pu}$ & $2.76 \mathrm{E}+01$ & $2.76 E-02$ & $2.9 E+03$ & $9.5 E-06$ \\
\hline${ }^{241} \mathrm{Am}^{\mathrm{c}}$ & $4.17 E+00$ & $4.17 E-03$ & $5.5 E+01$ & $7.6 \mathrm{E}-05$ \\
\hline & & & Sum of Ratios & $9.9 \mathrm{E}-04$ \\
\hline
\end{tabular}

Notes:

Based on $700 \mathrm{~g}$ for ${ }^{235} \mathrm{U}$ for $\mathrm{TQ}$ value
2 Based on $450 \mathrm{~g}$ for ${ }^{239} \mathrm{Pu}$ for TQ value 
Table A-8 is derived from Table A-5 and provides the result per $\mathrm{kg}$ of Weasel Pit sludge without criticality concern, while Table A-9 provides the result if criticality is a concern.

Table A-8. K East Weasel Pit Sludge Contribution to Hazard Category 2 Threshold Quantity Per Kilogram Sludge (No Criticality Concern).

\begin{tabular}{|l|c|c|c|c|}
\hline \multicolumn{1}{|c|}{ Isotope } & pCi/g & Ci/Kg Sludge & $\begin{array}{c}\text { Category 2 TQ } \\
\text { [Ci\} }\end{array}$ & $\begin{array}{c}\text { Ratio of Total } \\
\text { Ci/ Cat. 2 TQ }\end{array}$ \\
\hline${ }^{3} \mathrm{H}$ & $1.27 \mathrm{E}-02$ & $1.27 \mathrm{E}-05$ & $3.0 \mathrm{E}+05$ & $4.2 \mathrm{E}-11$ \\
\hline${ }^{60} \mathrm{Co}$ & $1.03 \mathrm{E}+01$ & $1.03 \mathrm{E}-02$ & $1.9 \mathrm{E}+05$ & $5.4 \mathrm{E}-08$ \\
\hline${ }^{90} \mathrm{Sr}$ & $4.48 \mathrm{E}+01$ & $4.48 \mathrm{E}-02$ & $2.2 \mathrm{E}+04$ & $2.0 \mathrm{E}-06$ \\
\hline${ }^{137} \mathrm{Cs}$ & $8.56 \mathrm{E}+01$ & $8.56 \mathrm{E}-02$ & $8.9 \mathrm{E}+04$ & $9.6 \mathrm{E}-07$ \\
\hline${ }^{154} \mathrm{Eu}$ & $9.51 \mathrm{E}+00$ & $9.51 \mathrm{E}-03$ & $1.1 \mathrm{E}+05$ & $8.6 \mathrm{E}-08$ \\
\hline${ }^{155} \mathrm{Eu}$ & $8.78 \mathrm{E}+00$ & $8.78 \mathrm{E}-03$ & $7.3 \mathrm{E}+05$ & $1.2 \mathrm{E}-08$ \\
\hline${ }^{234} \mathrm{U}$ & $1.37 \mathrm{E}-01$ & $1.37 \mathrm{E}-04$ & $2.2 \mathrm{E}+02$ & $6.2 \mathrm{E}-07$ \\
\hline${ }^{235} \mathrm{U}$ & $1.31 \mathrm{E}-02$ & $1.31 \mathrm{E}-05$ & $2.4 \mathrm{E}+02$ & $5.5 \mathrm{E}-08$ \\
\hline${ }^{238} \mathrm{Pu}$ & $8.11 \mathrm{E}+00$ & $8.11 \mathrm{E}-03$ & $6.2 \mathrm{E}+01$ & $1.3 \mathrm{E}-04$ \\
\hline${ }^{239 / 240} \mathrm{Pu}$ & $2.90 \mathrm{E}+01$ & $2.90 \mathrm{E}-02$ & $5.6 \mathrm{E}+01$ & $5.2 \mathrm{E}-04$ \\
\hline${ }^{241} \mathrm{Pu}$ & $5.90 \mathrm{E}+01$ & $5.90 \mathrm{E}-02$ & $2.9 \mathrm{E}+03$ & $2.0 \mathrm{E}-05$ \\
\hline${ }^{241} \mathrm{Am}{ }^{3}$ & $6.48 \mathrm{E}+00$ & $6.48 \mathrm{E}-03$ & $5.5 \mathrm{E}+01$ & $1.2 \mathrm{E}-04$ \\
\hline & & & Sum of Ratios & $7.9 \mathrm{E}-04$ \\
\hline
\end{tabular}

Table A-9. K East Weasel Pit Sludge Contribution to Hazard Category 2

Threshold Quantity Per Kilogram Sludge (With Criticality Concern).

\begin{tabular}{|c|c|c|c|c|}
\hline Isotope & $\mu \mathrm{Ci} / \mathrm{g}$ & Ci/Kg Sludge & $\begin{array}{c}\text { Category } 2 \mathrm{TQ} \\
\text { [Cl] }\end{array}$ & $\begin{array}{l}\text { Ratio of Total } \\
\text { Cit Cat } 2 \text { ro }\end{array}$ \\
\hline${ }^{3} \mathrm{H}$ & 1.27E-02 & $1.27 \mathrm{E}-05$ & $3.0 E+05$ & $4.2 \mathrm{E}-11$ \\
\hline${ }^{60} \mathrm{Co}$ & $1.03 E+01$ & $1.03 \mathrm{E}-02$ & $1.9 E+05$ & $5.4 \mathrm{E}-08$ \\
\hline${ }^{90} \mathrm{Sr}$ & $4.48 E+01$ & $4.48 \mathrm{E}-02$ & $2.2 E+04$ & 2.0E-06 \\
\hline${ }^{137} \mathrm{Cs}$ & $8.56 \mathrm{E}+01$ & $8.56 \mathrm{E}-02$ & $8.9 E+04$ & $9.6 \mathrm{E}-07$ \\
\hline${ }^{154} \mathrm{Eu}$ & $9.51 E+00$ & $9.51 \mathrm{E}-03$ & $1.1 E+05$ & $8.6 \mathrm{E}-08$ \\
\hline${ }^{155} \mathrm{Eu}$ & $8.78 E+00$ & $8.78 E-03$ & $7.3 E+05$ & $1.2 \mathrm{E}-08$ \\
\hline${ }^{234} \mathrm{U}$ & 1.37E-01 & $1.37 \mathrm{E}-04$ & $2.2 \mathrm{E}+\mathrm{O} 2$ & $6.2 \mathrm{E}-07$ \\
\hline${ }^{235} \mathrm{U}$ & $1.31 \mathrm{E}-\mathrm{O} 2$ & $1.31 \mathrm{E}-05$ & $1.51 \mathrm{E}-03^{1}$ & $8.7 \mathrm{E}-03$ \\
\hline${ }^{238} \mathrm{Pu}$ & $8.11 E+00$ & $8.11 \mathrm{E}-03$ & $6.2 \mathrm{E}+01$ & $1.3 \mathrm{E}-04$ \\
\hline${ }^{239 / 240} \mathrm{Pu}$ & $2.90 E+01$ & $2.90 \mathrm{E}-02$ & $2.8 \mathrm{E}+01^{2}$ & 1.0E-03 \\
\hline${ }^{241} \mathrm{Pu}$ & $5.90 \mathrm{E}+01$ & $5.90 \mathrm{E}-02$ & $2.9 \mathrm{E}+03$ & 2.0E-05 \\
\hline${ }^{241} \mathrm{Am}^{3}$ & $6.48 \mathrm{E}+00$ & $6.48 \mathrm{E}-03$ & $5.5 \mathrm{E}+01$ & 1.2E-04 \\
\hline & & & Sum of Ratios & 1.OE-02 \\
\hline
\end{tabular}

\footnotetext{
${ }^{1}$ Based on $700 \mathrm{~g}$ for ${ }^{235} \mathrm{U}$ for TQ value

${ }^{2}$ Based on $450 \mathrm{~g}$ for ${ }^{239} \mathrm{Pu}$ for TQ value
} 


\section{A.1.3 KE Ion Exchange Modules (IXM)}

The IXM isotopic loading shown in Table A-10 represents the approximate $90 \%$ maximum of TRU that is allowed before being classified as TRU waste. Currently, the IXMs are changed out upon reaching $90 \%$ of the maximum TRU loading of $100 \mathrm{nCi} / \mathrm{g}$. This results in approximately $1.7 \mathrm{Ci}$ of TRU (i.e., ${ }^{239} \mathrm{Pu}$ ) per IXM. An accident scenario previously exiamined in (Porten and Crowe 1994) and (Marusich 1994) was a radiological release from an IXM due to an explosion of hydrogen gas from radiolysis of the contained resin. Marusich (1994) analyzed an IXM that contained $265 \mathrm{Ci}$ of ${ }^{137} \mathrm{Cs}, 104 \mathrm{Ci}$ of ${ }^{90} \mathrm{Sr}$, and $1.01 \mathrm{Ci}$ of TRU. Tables $\mathrm{A}-10$ and $\mathrm{A}-11$ show the results if the other two isotopes $\left({ }^{137} \mathrm{Cs}\right.$ and ${ }^{90} \mathrm{Sr}$ ) are increased by a ratio of 1.7 of the values used by (Marusich 1994). The U/Pu weight ratio is assumed to be at 400 , which is typical for KE basin water. At a specific activity of ${ }^{239} \mathrm{Pu}$ of $0.062 \mathrm{Ci} / \mathrm{g}, 1.7 \mathrm{Ci}$ of ${ }^{239} \mathrm{Pu}$ represents $27.4 \mathrm{~g}$ of ${ }^{239} \mathrm{Pu}$. Therefore, there would be $400 \times 27.4 \mathrm{~g}=1.10 \mathrm{E}+04 \mathrm{~g}$ of uranium. With an enrichment level of $0.947 \mathrm{wt} \%$, there would be $103.8 \mathrm{~g}$ of ${ }^{235} \mathrm{U}$. With a specific activity of ${ }^{235} \mathrm{U}$ of $2.16 \mathrm{E}-06 \mathrm{Ci} / \mathrm{g}, 2.24 \mathrm{E}-04 \mathrm{Ci}$ of ${ }^{235} \mathrm{U}$ exist in the $\mathrm{IXM}$. The $\mathrm{Ci}$ of ${ }^{238} \mathrm{U}$ is based upon a specific activity of $3.36 \mathrm{E}-07 \mathrm{Ci} / \mathrm{g}$. Table A-12 displays the result of 1,250 canisters (six months of de-lidding operations) in $\mathrm{KW}$ with a loading of $0.286 \mathrm{Ci}$ of ${ }^{137} \mathrm{Cs}$ per canister in an IXM with no criticality concern, and Table A-13 displays the result with criticality concern.

Table A-10. Isotopic Loading For One KE Ion Exchange Module and Comparison to Hazard Category Threshold Quantity 2 Values (No Criticality Concern)

\begin{tabular}{|l|c|c|c|}
\hline \multicolumn{1}{|c|}{ Isotope } & $\begin{array}{c}\mathrm{Ci} \text { per IXM } \\
\text { Category 2 TO }\end{array}$ & $\begin{array}{c}\text { Ratio of Total Cil } \\
\text { Cat 2 TO }\end{array}$ \\
\hline${ }^{90} \mathrm{Sr}$ & $1.8 \mathrm{E}+02$ & $2.2 \mathrm{E}+04$ & $8.2 \mathrm{E}-03$ \\
\hline${ }^{137} \mathrm{Cs}$ & $4.5 \mathrm{E}+02$ & $8.9 \mathrm{E}+04$ & $5.1 \mathrm{E}-03$ \\
\hline${ }^{239} \mathrm{Pu}$ & $1.7 \mathrm{E}+00$ & $5.6 \mathrm{E}+01$ & $3.0 \mathrm{E}-02$ \\
\hline${ }^{235} \mathrm{U}$ & $2.24 \mathrm{E}-04$ & $2.4 \mathrm{E}+02$ & $9.3 \mathrm{E}-07$ \\
\hline${ }^{238} \mathrm{U}$ & $3.66 \mathrm{E}-03$ & $2.4 \mathrm{E}+02$ & $1.5 \mathrm{E}-05$ \\
\hline & & Sum of Ratios & $4.3 \mathrm{E}-02$ \\
\hline
\end{tabular}

Table A-11. Isotopic Loading For One KE Ion Exchange Module and Comparison to Hazard Category Threshold Quantity 2 Values (With Criticality Concern)

\begin{tabular}{|l|c|c|c|}
\hline \multicolumn{1}{|c|}{ 1sotope } & Ci per IXM & Category 2 Ta & Ratio of Total Cil Cat $_{2} \mathrm{TO}$ \\
\hline${ }^{90} \mathrm{Sr}$ & $1.8 \mathrm{E}+02$ & $2.2 \mathrm{E}+04$ & $8.2 \mathrm{E}-03$ \\
\hline${ }^{137} \mathrm{Cs}$ & $4.5 \mathrm{E}+02$ & $8.9 \mathrm{E}+04$ & $5.1 \mathrm{E}-03$ \\
\hline${ }^{239} \mathrm{Pu}$ & $1.7 \mathrm{E}+00$ & $2.8 \mathrm{E}+01^{1}$ & $6.1 \mathrm{E}-02$ \\
\hline${ }^{235} \mathrm{U}$ & $2.24 \mathrm{E}-04$ & $1.51 \mathrm{E}-03^{2}$ & $1.5 \mathrm{E}-01$ \\
\hline${ }^{238} \mathrm{U}$ & $3.66 \mathrm{E}-03$ & $2.4 \mathrm{E}+02$ & $1.5 \mathrm{E}-05$ \\
\hline
\end{tabular}

${ }^{1}$ Based on $450 \mathrm{~g}$ for ${ }^{239} \mathrm{Pu}$ for TQ value

${ }^{2}$ Based on $700 \mathrm{~g}$ for ${ }^{235} \mathrm{U}$ for TO value 
Table A-12. Isotopic Loading For One KW Ion Exchange Module With Increased ${ }^{137} \mathrm{Cs}$ Loading and Comparison to Category Threshold Quantity 2 Values

(No Criticality Concern)

\begin{tabular}{|l|c|c|c|}
\hline \multicolumn{1}{|c|}{ Isotope } & Ci per IXM & Category 2 TO & $\begin{array}{c}\text { Ratio of Total } \\
\text { Ci/Cat 2 TO }\end{array}$ \\
\hline${ }^{90} \mathrm{Sr}$ & $1.8 \mathrm{E}+02$ & $2.2 \mathrm{E}+04$ & $8.2 \mathrm{E}-03$ \\
\hline${ }^{137} \mathrm{Cs}$ & $8.1 \mathrm{E}+02$ & $8.9 \mathrm{E}+04$ & $9.1 \mathrm{E}-03$ \\
\hline${ }^{239} \mathrm{Pu}$ & $1.7 \mathrm{E}+00$ & $5.6 \mathrm{E}+01$ & $3.0 \mathrm{E}-02$ \\
\hline${ }^{235} \mathrm{U}$ & $2.24 \mathrm{E}-04$ & $2.4 \mathrm{E}+02$ & $9.3 \mathrm{E}-07$ \\
\hline${ }^{238} \mathrm{U}$ & $3.66 \mathrm{E}-03$ & $2.4 \mathrm{E}+02$ & $1.5 \mathrm{E}-05$ \\
\hline & & Sum of Ratios & $4.7 \mathrm{E}-02$ \\
\hline
\end{tabular}

Table A-13. Isotopic Loading For One KW Ion Exchange Module (KW) With Increased ${ }^{137} \mathrm{Cs}$ Loading and Comparison to Hazard Category Threshold Quantity 2 Values (With Criticality Concern)

\begin{tabular}{|l|c|c|c|}
\hline \multirow{2}{*}{$\begin{array}{l}\text { Isotope } \\
{ }^{90} \mathrm{Sr}\end{array}$} & Ci per IXM & Category 2 TO & $\begin{array}{c}\text { Ratio of Total } \\
\text { Ci/Cat 2 TO }\end{array}$ \\
\hline${ }^{137} \mathrm{Cs}$ & $1.8 \mathrm{E}+02$ & $2.2 \mathrm{E}+04$ & $8.2 \mathrm{E}-03$ \\
\hline${ }^{239} \mathrm{Pu}$ & $8.1 \mathrm{E}+02$ & $8.9 \mathrm{E}+04$ & $9.1 \mathrm{E}-03$ \\
\hline${ }^{235} \mathrm{U}$ & $1.7 \mathrm{E}+00$ & $2.8 \mathrm{E}+01^{\prime}$ & $6.1 \mathrm{E}-02$ \\
\hline${ }^{238} \mathrm{U}$ & $2.24 \mathrm{E}-04$ & $1.51 \mathrm{E}-03^{2}$ & $1.5 \mathrm{E}-01$ \\
\hline & $3.66 \mathrm{E}-03$ & $2.4 \mathrm{E}+02$ & $1.5 \mathrm{E}-05$ \\
\hline
\end{tabular}

\footnotetext{
'Based on $450 \mathrm{~g}$ for ${ }^{239} \mathrm{Pu}$ for TQ value
}

${ }^{2}$ Based on $700 \mathrm{~g}$ for ${ }^{235} \mathrm{U}$ for TQ value

\section{A.1.4 K West Fuel}

There are approximately 3,815 canisters in $\mathrm{KW}$ containing 50,683 fuel assemblies. The total fuel mass in the basin is 954 metric tons of heavy metals. The average Mark IA fuel assembly has a uranium weight of $16.3 \mathrm{~kg}$, less than the average KE fuel assembly of $22.7 \mathrm{~kg}$ uranium. The contribution of one average $\mathrm{KW}$ fuel assembly to the Category 2 TO without criticality concern is displayed in Table A-14, while with criticality concern is shown in Table A-15. 
Table A-14. Isotopic Content of One Average Fuel Assembly (KW, No Criticality Concern).

\begin{tabular}{|c|c|c|c|}
\hline Isotope & Ci per Assembly & $\begin{array}{c}\text { Category } 2 \mathrm{TQ} \\
\text { (Ci) }\end{array}$ & $\begin{array}{c}\text { Ratio of Total Ci/Cat. } \\
\qquad 2 \mathrm{TO}\end{array}$ \\
\hline${ }^{90} \mathrm{Sr}$ & $1.28 E+02$ & $2.2 E+04$ & $5.8 \mathrm{E}-03$ \\
\hline${ }^{137} \mathrm{Cs}$ & $1.76 E+02$ & $8.9 E+04$ & $2.0 E-03$ \\
\hline${ }^{235} \mathrm{U}$ & $4.05 \mathrm{E}-04$ & $2.4 E+02$ & $1.7 E-06$ \\
\hline${ }^{238} \mathrm{U}$ & $5.42 \mathrm{E}-03$ & $2.4 E+02$ & $2.3 E-05$ \\
\hline${ }^{238} \mathrm{Pu}$ & $2.78 \mathrm{E}+00$ & $6.2 \mathrm{E}+01$ & $4.5 \mathrm{E}-\mathrm{O} 2$ \\
\hline${ }^{239} \mathrm{Pu}$ & $2.58 E+00$ & $5.6 E+01$ & $4.6 \mathrm{E}-02$ \\
\hline${ }^{240} \mathrm{Pu}$ & $2.09 E+00$ & $5.5 E+01$ & $3.8 \mathrm{E}-\mathrm{O} 2$ \\
\hline${ }^{241} \mathrm{Pu}$ & $1.51 \mathrm{E}+02$ & $2.9 E+03$ & $5.2 \mathrm{E}-02$ \\
\hline${ }^{242} \mathrm{Pu}$ & $1.71 \mathrm{E}-03$ & $2.8 \mathrm{E}+01$ & $6.1 \mathrm{E}-05$ \\
\hline${ }^{241} \mathrm{Am}$ & $5.84 E+00$ & $5.5 E+01$ & $1.1 \mathrm{E}-01$ \\
\hline & & Sum of Ratios & 3.0E-01 \\
\hline
\end{tabular}

Table A-15. Isotopic Content of One Average Fuel Assembly (KW, With Criticality Concern).

\begin{tabular}{|l|c|c|c|}
\hline \multicolumn{1}{|c|}{ Isotope: } & Ci per Assembly & $\begin{array}{c}\text { Category 2 TO } \\
\text { (Ci) }\end{array}$ & $\begin{array}{c}\text { Ratio of Total } \\
\text { Ci/Cat 2 TO }\end{array}$ \\
\hline${ }^{90} \mathrm{Sr}$ & $1.28 \mathrm{E}+02$ & $2.2 \mathrm{E}+04$ & $5.8 \mathrm{E}-03$ \\
\hline${ }^{137} \mathrm{Cs}$ & $1.76 \mathrm{E}+02$ & $8.9 \mathrm{E}+04$ & $2.0 \mathrm{E}-03$ \\
\hline${ }^{235} \mathrm{U}$ & $4.05 \mathrm{E}-04$ & $1.51 \mathrm{E}-03^{1}$ & $2.7 \mathrm{E}-01$ \\
\hline${ }^{238} \mathrm{U}$ & $5.42 \mathrm{E}-03$ & $2.4 \mathrm{E}+02$ & $2.3 \mathrm{E}-05$ \\
\hline${ }^{238} \mathrm{Pu}$ & $2.78 \mathrm{E}+00$ & $6.2 \mathrm{E}+01$ & $4.5 \mathrm{E}-02$ \\
\hline${ }^{239} \mathrm{Pu}$ & $2.58 \mathrm{E}+00$ & $2.8 \mathrm{E}+01^{2}$ & $9.2 \mathrm{E}-02$ \\
\hline${ }^{240} \mathrm{Pu}$ & $2.09 \mathrm{E}+00$ & $5.5 \mathrm{E}+01$ & $3.8 \mathrm{E}-02$ \\
\hline${ }^{241} \mathrm{Pu}$ & $1.51 \mathrm{E}+02$ & $2.9 \mathrm{E}+03$ & $5.2 \mathrm{E}-02$ \\
\hline${ }^{242} \mathrm{Pu}$ & $1.71 \mathrm{E}-03$ & $2.8 \mathrm{E}+01$ & $6.1 \mathrm{E}-05$ \\
\hline${ }^{241} \mathrm{Am}$ & $5.84 \mathrm{E}+00$ & $5.5 \mathrm{E}+01$ & $1.1 \mathrm{E}-01$ \\
\hline & & Sum of Ratios & $6.1 \mathrm{E}-01$ \\
\hline
\end{tabular}

Note:

\footnotetext{
1 Based on $700 \mathrm{~g}$ for ${ }^{235} \mathrm{U}$ for to value

${ }^{2}$ Based on $450 \mathrm{~g}$ for ${ }^{239} \mathrm{Pu}$ for TQ value
} 


\section{REFERENCES}

Marusich, 1994, Hydrogen Production in the $K$ Basin Ion Exchange Columns, Modules and Cartridge Filters, WHC-SD-SNF-SARR-003, Rev. O, Westinghouse Hanford Company, Richland, Washington.

Porten and Crowe, 1994, $K$ Basin Safety Analysis, WHC-SD-SNF-RA-001, Rev. O, Westinghouse Hanford Company, Richland, Washington.

WHC, 1995, Spent Nuclear Fuel Project Technical Databook, WHC-SD-SNF-TI-015, Rev. 0. Westinghouse Hanford Company, Richland, Washington.

Willis, 1995, 105-K Basin Material Design Basis Feed Description For Spent Nuclear Fuel Project Facilities, WHC-SD-SNF-TI-009, Westinghouse Hanford Company, Richland, Washington. 
WHC-SD-SNF-RPT-012, Rev. 0

\section{APPENDIX C}

\section{COST ESTIMATE}

C-1 
WHC-SD-SNF-RPT-012, Rev. 0

I CF KAISER HAHFORD

WEST INGHOUSE HANFORD COMPANY

JOB NO E $57991 / 2609$

FILE NO, 2409PAA3
* * IEST - INTERACTIVE ESTIMATING * *

K-BASINS INTEGRATEO HATER SYSTEM

DOE_RO1 - PRELIMINARY
PAGE 1 OF 15

DATE $07 / 2419619: 28: 27$

BY JPM SMF DKH LGH

\section{$\cos T$ \\ CODE \\ $== \pm=$ \\ 010 \\ 020 \\ 030 \\ 060 \\ DE SCR I P T I ON \\ TITLE I DESIGN \\ IITLE II DESIGN \\ TITLE III CONST. ENG/INSPECTION \\ PROJECT MANAGEMENT \\ (ADJUSTED TO MEET DOE 5100.4) \\ TOTAL DESIGN MANAGEMENT

$\begin{array}{ll}501 & \text { BUILDINGS } \\ 550 & \text { OTHER STRUCTURES } \\ 700 & \text { SPECIAL EQUIP/PROCESS SYSTEMS } \\ & \text { (ADJUSTED TO MEET DOE } 5100.4 \text { ) }\end{array}$

TOTAL CONSTRUCTION COST

TOTAL ESTIMATED CONSTRUCTION COST (TEEC)

900 OTHER PROJECT COSTS

(ADJUSTED TO MEET DOE 5100.4)

TOTAL OTHER PROJECT COSTS

TOTAL PROJECT COST

(TPC)

\begin{tabular}{|c|c|c|c|}
\hline $\begin{array}{l}\text { ESCALATED } \\
\text { TOTAL COST } \\
========\end{array}$ & $==\stackrel{\%^{\operatorname{CON}}}{==}$ & $\begin{array}{l}\text { UGENCY } \\
\text { TOTAL } \\
=========\end{array}$ & $\begin{array}{c}\text { TOIAL } \\
\text { OOLLARS } \\
==========8\end{array}$ \\
\hline $\begin{array}{r}240,000 \\
1,600,000 \\
420,000 \\
820,000 \\
20,000\end{array}$ & $\begin{array}{l}0 \\
10 \\
30 \\
10\end{array}$ & $\begin{array}{r}0 \\
160,000 \\
130,000 \\
80,000 \\
30,000\end{array}$ & $\begin{array}{r}240,000 \\
1,760,000 \\
550,000 \\
900,000 \\
50,000\end{array}$ \\
\hline $\begin{array}{r}======== \\
3,100,000\end{array}$ & $=== \pm=z=$ & 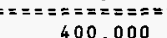 & $=== \pm====2=$ \\
\hline $\begin{array}{r}1,430,000 \\
240,000 \\
4,370,000 \\
-40,000 \\
=======0 \\
6,000,000\end{array}$ & $===\begin{aligned} 23 \\
\end{aligned}$ & $=\begin{array}{r}360,000 \\
50,000 \\
1,020,000 \\
.30,000 \\
1,400,000\end{array}$ & $\begin{array}{r}1,790,000 \\
290,000 \\
5,390,000 \\
-70,000 \\
========= \\
7,400,000\end{array}$ \\
\hline $9,100,000$ & 20 & $1,800,000$ & $10,900,000$ \\
\hline $\begin{array}{r}2,510,000 \\
-10,000\end{array}$ & $\begin{array}{l}0 \\
0\end{array}$ & $\begin{array}{l}0 \\
0\end{array}$ & $\begin{array}{r}2,590,000 \\
-10,000\end{array}$ \\
\hline $\begin{aligned} &======== \\
&== \\
& 2,500,000\end{aligned}$ & $\begin{aligned} &=z==\Sigma \\
& 0\end{aligned}$ & $\begin{array}{r}=\boldsymbol{=}=\boldsymbol{=}=\boldsymbol{=}=\boldsymbol{=}=\boldsymbol{=} \\
0\end{array}$ & $\begin{aligned}==x====== \\
2,500,000\end{aligned}$ \\
\hline $11,600,000$ & 15 & $1,800,000$ & $13,400,000$ \\
\hline
\end{tabular}

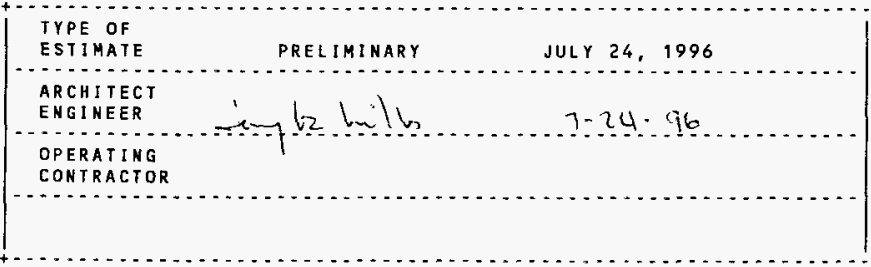

REMARKS :

(ROUNDED/ADJUSTED TO THE NEAREST"10,000/100,000" PERCENTAGES NOT RECALCULATED TO REFLECT ROUNDING) 
WHC-SD-SNF-RPT-012, Rev. 0

ICF KAISER HANFORD

WESTINGHOUSE HANFORD COMPANY

JOB NO. E57991/Z409

FILE NO. Z 409 PAA3

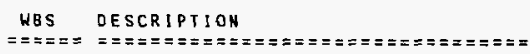

112i0O TITLE I DEF/DESIGN - OFFSITE A/E

112210 TITLE II DEF/DESIGN - OFFSITE A/E

SUBTOTAL 112 DEFINITIVE DESIGN

SUBTOTAL 11 DEFINITIVE DESIGN

122000 ENGINEERING/INSPECTION-OFFSITE A/E

SUBTOTAL 122 ENGR, IINSP. OFFSITE A/E

SUBTOTAL 12 ENGINEERING/INSPECTION

SUBTOTAL 1

ENG INEER ING

220001 K-EAST IXM PROCUREMENT

220002 K-WEST IXM PROCUREMENT

220003 K-EAST BACKWASH FILTERS

$\begin{array}{ll}\text { SUBTOTAL } 22 & \text { PROCUREMENT-O/C } \\ \text { SUBTOTAL } 2 & \text { PROCUREMENT }\end{array}$

310100 MOCK-UP FACILITIES \& TRAINING

310101 CHILLER BAY MODIFICATIONS

310102 PRIMARY CLEANING STATION PIPING

310103 DOWN DRAFT TABLE PIPING

310104 MISC DRAIN VENT \& RETURN TO BASIN

310106 DEBRIS REMOVAL PIPINO

310107 WEASEL PIT PUMP \& PIPING

310108 HYDROCYCLONES \& PIPING

310109 FILTER BACKHASH PIPING

310110 SW LOADOUT PIPING

310111 DEMINERALIZED WATER
310112 WEASEL PIT MODIFICATIONS

310113 B/W FLTRS SUPPLY AND RETURN

310115 POWER WIRING

$\begin{array}{lll}310115 & \text { POHER WIRING } & \text { KE } \\ 310116 & \text { INSTRUMENTATION } & \text { KE }\end{array}$
* IEST - INIERACTIVE ESTIMATING **

K-BASINS IMTEGRATED HATER SYSTEM

DOE_RO2 - WORK BREAKDOWN STRUCTURE SUMMARY
DOLINARY
PAGE 2 OF 15

DATE $07 / 24 / 96 \quad 11.28: 31$

BY JPM SMF DKH LGH

\begin{tabular}{|c|c|c|c|c|c|c|c|c|}
\hline $\begin{array}{l}\text { ESTIMATE } \\
\text { SUB TOTAL } \\
======\end{array}$ & $\begin{array}{l}\text { ONSITE } \\
\text { INDIRECTS } \\
=======\end{array}$ & $\begin{array}{c}\text { SUB } \\
\text { TOTAL } \\
========\end{array}$ & 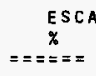 & $\begin{array}{l}\text { ALATION } \\
\text { TOTAL } \\
=======\end{array}$ & $\begin{array}{c}\text { SUB } \\
\text { TOTAL } \\
=======\end{array}$ & $\begin{array}{l}\operatorname{conTI} \\
====\end{array}$ & $\begin{array}{l}\text { NGENCY } \\
\text { IOIAL } \\
========\end{array}$ & $\begin{array}{c}\text { TOTAL } \\
\text { DOLLARS } \\
===== \pm= \pm\end{array}$ \\
\hline $\begin{array}{r}240000 \\
1600000\end{array}$ & $\begin{array}{l}0 \\
0\end{array}$ & $\begin{array}{r}240000 \\
+600000\end{array}$ & $\begin{array}{l}0.00 \\
0.00\end{array}$ & $\begin{array}{l}0 \\
0\end{array}$ & $\begin{array}{r}240000 \\
1600000\end{array}$ & $\begin{array}{r}0 \\
10\end{array}$ & $\begin{array}{r}0 \\
160000\end{array}$ & $\begin{array}{r}240000 \\
1760000\end{array}$ \\
\hline 1840000 & 0 & 1840000 & 0.00 & 0 & 1840000 & 9 & $\$ 60000$ & 2000000 \\
\hline 1840000 & 0 & 1840000 & 0.00 & 0 & 1840000 & 9 & 160000 & 2000000 \\
\hline 413959 & 0 & 413959 & 1.71 & 7079 & 421038 & 30 & 126311 & 547349 \\
\hline 413959 & 0 & 413959 & 1.71 & 7079 & 421038 & 30 & 126311 & 547349 \\
\hline 413959 & 0 & 413959 & 1.71 & 7079 & 421038 & 30 & 926311 & 547349 \\
\hline 2253959 & 0 & 2253959 & 0.31 & 7079 & 2261038 & 13 & 286311 & 2547349 \\
\hline $\begin{array}{r}155775 \\
93465 \\
588206\end{array}$ & $\begin{array}{l}0 \\
0 \\
0\end{array}$ & $\begin{array}{r}155775 \\
93465 \\
588206\end{array}$ & $\begin{array}{l}1.71 \\
1.71 \\
1.71\end{array}$ & $\begin{array}{r}2664 \\
1598 \\
10058\end{array}$ & $\begin{array}{r}158439 \\
95063 \\
598264\end{array}$ & $\begin{array}{l}15 \\
20 \\
30\end{array}$ & $\begin{array}{r}23766 \\
19013 \\
179479\end{array}$ & $\begin{array}{l}182205 \\
114076 \\
777744\end{array}$ \\
\hline 837446 & 0 & 837446 & 1.71 & 14320 & 851766 & 26 & 222258 & 1074025 \\
\hline 837446 & 0 & 837446 & 1.71 & 14320 & 851766 & 26 & 222258 & 1074025 \\
\hline $\begin{array}{r}142236 \\
134503 \\
63935 \\
78391 \\
13890 \\
74923 \\
70579 \\
135964 \\
76996 \\
90556 \\
15422 \\
409110 \\
307131 \\
82865 \\
518604\end{array}$ & $\begin{array}{l}0 \\
0 \\
0 \\
0 \\
0 \\
0 \\
0 \\
0 \\
0 \\
0 \\
0 \\
0 \\
0 \\
0 \\
0\end{array}$ & $\begin{array}{r}142236 \\
134503 \\
63935 \\
78391 \\
13890 \\
74923 \\
70579 \\
135964 \\
76996 \\
90556 \\
15422 \\
409110 \\
307131 \\
82865 \\
518604\end{array}$ & $\begin{array}{l}1.71 \\
1.71 \\
1.71 \\
1.71 \\
1.71 \\
1.71 \\
1.71 \\
1.71 \\
1.71 \\
1.71 \\
1.71 \\
1.71 \\
1.71 \\
1.71 \\
1.71\end{array}$ & $\begin{array}{r}2432 \\
2300 \\
1093 \\
1341 \\
238 \\
1282 \\
1208 \\
2325 \\
1317 \\
1548 \\
263 \\
6996 \\
5252 \\
1418 \\
8869\end{array}$ & $\begin{array}{r}144668 \\
136803 \\
65028 \\
79732 \\
14128 \\
76205 \\
71787 \\
138289 \\
78313 \\
92104 \\
15685 \\
416106 \\
312383 \\
84283 \\
527473\end{array}$ & $\begin{array}{l}20 \\
27 \\
20 \\
20 \\
27 \\
21 \\
21 \\
20 \\
20 \\
20 \\
20 \\
23 \\
20 \\
25 \\
25\end{array}$ & $\begin{array}{r}28934 \\
36816 \\
13005 \\
15946 \\
3822 \\
16168 \\
15212 \\
27658 \\
15662 \\
18421 \\
3137 \\
97271 \\
62477 \\
21071 \\
134029\end{array}$ & $\begin{array}{r}173602 \\
173619 \\
78034 \\
95678 \\
17950 \\
92373 \\
86998 \\
165946 \\
93975 \\
110526 \\
18823 \\
513377 \\
374860 \\
105352 \\
661500\end{array}$ \\
\hline
\end{tabular}


WHC-SD-SNF-RPT-012, Rev . 0

ICF KAISER HANFORD

WESTINGHOUSE HANFORD COMPANY

JOB NO. E $57991 / 2409$

FILE NO. Z4O9PAA3

WBS DESCRIPTION

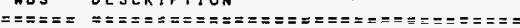

310117 STORAGE SLAB PREP

310118 CHILLER \& HEAT EXCHAHGER DEMOLITION

390119 PAINT AND LABEL PIPING

310180 BURNOUT

310190 SUPPORT REQUIREMENTS

310191 NEW RAD STORAGE PAD

SUBTOTAL 3101 K-EAST BASIN MOOIFICATIONS

310300 MOCK-UP FACILITIES \& TRAINING

310307 NEH RECIRC PUMPS

310308 TRANSFER BAY MODIFICATIONS

310309 DELID STATION

310310 PRIMARY CLEAN STATION

310311 DOWN DRAFT TABLE \& DRY CANISTER STA

310312 DEBRIS REMOVAL

310313 SH LOAD OUT PIT

310314 DEMINERALIZED HATER

310315 POWER HIRING

KW

310316 INSTRUMENTATION KH

310317 PAINI AND LABEL PIPING

310390 SUPPORT REQUIREMENTS

SUBTOTAL 3103 K-WEST BASIH MODIFICATIONS

SUBTOTAL 31 FA CONST-ONSITE E/C

321200 PRE-ENGINEERED METAL BUILDING

321201 IXM SUPPLY \& RETURH PIPING

321202 IXM DRAIN \& VENT

321204 COMP/AIR AND SAFETY SHOWER

321205 HVAC \& HEPA FILTRATIOH

SUBTOTAL 3212 K-EAST AHNEX

SUBTOTAL 32 CONSTRUCTION-FIXED PRICE

330000 BURIAL FEES
* * IEST - INTERACTIVE ESTIMATING * *

$K$-BASINS INTEGRATED HATER SYSTEM PRELIMINARY

DOE_RO2 - HORK BREAKDOWN STRUCTURE SUMMARY

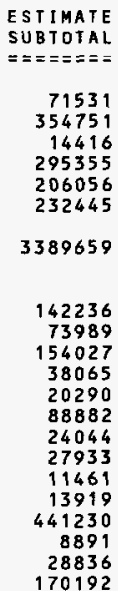

1243995

4633654

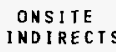

INDIRECIS

SUB

TOTAL
$====+=$

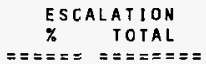

$=== \pm==$

71531
354751
14416

14416
295355

206056

1.71
1.71
1.71

1.71

1.71

232445

1.71

3389659

1.7

142236

73989

154027

38065

20290

88882
24044

24044
27933

11461

13919

441230

8891

28836
170192

1. 71

1.71
1.71
1.71

1.71

1.7

1.71

1.71
1.71

1.71
1.71

9.71

1. 71

1.71

1.71

1.71

1243995

1.71

4633654

1.7

1223
6066

6066
247

5051

3524
3975

57968

2432

1265
2634

2634

651
347

1520

1520
411

478

196
238

7545
152

493

493
2910

21272

79240

136249

136249
54347

50522

26963

22000

290081

290081

94380 $\begin{array}{lll}177267 & 1.71 & 3031\end{array}$

67716

62950

33596

27412

1.71

1.71

1.71
1.71

1.71

78860

368941

368941

1.71

94380

1.71

1158
1076

1076
574

469

6308

6308

1614

SUB

CONT INGENCY

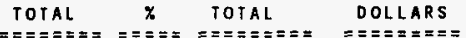

$\begin{array}{rr}72754 & 20 \\ 360817 & 30\end{array}$

1466320

$300406 \quad 30$

$\begin{array}{ll}209580 & 20 \\ 236420 & 20\end{array}$

$3447627 \quad 24$

14551

108245
2933

2933
90122

41916

47283

814679

144668
75254

75254
156661

38716

20637

90402

24455

28411

11657

14157

448775

9043

29329

173102

1265267

4712894

20

16

28934

12321

31959
7743

7143
4127

18081

18081

4891

5682
2331

3539

111809

1809

8799

34620

276645

1091324

87305

469062

17595

390527

251495

283704

4262301

180297

68874

64027

34170

27881

375249

375249

50782

13775

12805

6834

5576

173602

87575

188621

46459

24764

108483

20346

29346

34093
13988

13988

17696

560584

10852

38128
207723

1541914

5804215

231079

82649
76832

41004

33457

89772

465021

89772

465021

28798

124792 
WHC-SD-SNF-RPT-012, Rev. 0

ICF KAISER HANFORO

WESTINGHOUSE HANFORD COMPANY

JOB NO. E57991/2409

FILE NO. Z4O9PAA3

WBS DESCRIPIION

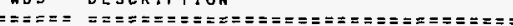

SUBTOTAL 33 CONSTRUCTION D/C

SUBTOTAL 3 CONSTRUCTION

400000 PROJECT MANAGEMENT

SUBTOTAL 4 PROJECT INTEGRATION

500096 FY 96 OTHER PROJECT COST

500097 FY 97 OTHER PROJECT COST

500098 FY 98 OTHER PROJECT COST

SUBTOTAL 5 OTHER PROJECT COST

PROJECT TOTAL
* * IEST - INTERACTIVE ESTIMATING * *

K-BASINS INTEGRATED WATER SYSTEM$$
\text { PRELIMINARY }
$$

DOE_RO2 - WORK BREAKDOWN STRUCTURE SUMMARY

$$
\begin{array}{ll}
\text { PAGE } & 4 \text { OF } 15 \\
\text { DATE } & 07 / 24 / 9611: 28: 31 \\
\text { BY } & \text { JPM SMF DKH LGH }
\end{array}
$$

\begin{tabular}{|c|c|c|c|c|c|c|c|c|}
\hline $\begin{array}{l}\text { EST IMATE } \\
\text { SUB TOTAL } \\
=======\end{array}$ & $\begin{array}{l}\text { ONSITE } \\
\text { IND IRECTS } \\
=======\end{array}$ & $\begin{array}{c}\text { SUB } \\
\text { IOT AL } \\
=======\end{array}$ & $\begin{aligned} & E S C A \\
& \% \\
&==\equiv \equiv=\end{aligned}$ & $\begin{array}{l}\text { ALATION } \\
\text { TOTAL } \\
=======\end{array}$ & $\begin{array}{c}\text { SUB } \\
\text { TOTAL } \\
=======\end{array}$ & $\begin{aligned} \text { CONTI } & \underset{*}{*}==\end{aligned}$ & $\begin{array}{l}\text { INGENCY } \\
\text { TOTAL } \\
========\end{array}$ & $\begin{array}{c}\text { TOTAL } \\
\text { DOLLARS } \\
=======\end{array}$ \\
\hline 94380 & 0 & 94380 & 1.71 & 1614 & 95994 & 30 & 28798 & 124792 \\
\hline 5018115 & 78860 & 5096975 & 1.71 & 87162 & 5184137 & 23 & 1209894 & 6394028 \\
\hline $8 \$ 1000$ & 0 & 811000 & 0.67 & 5434 & 816434 & 10 & 81643 & 898077 \\
\hline 811000 & 0 & 811000 & 0.67 & 5434 & 816434 & 10 & 81643 & 898077 \\
\hline $\begin{array}{r}527991 \\
1105000 \\
874018\end{array}$ & $\begin{array}{l}0 \\
0 \\
0\end{array}$ & $\begin{array}{r}527991 \\
1105000 \\
874018\end{array}$ & $\begin{array}{l}0.00 \\
0.00 \\
0.00\end{array}$ & $\begin{array}{l}0 \\
0 \\
0\end{array}$ & $\begin{array}{r}527991 \\
1105000 \\
874018\end{array}$ & $\begin{array}{l}0 \\
0 \\
0\end{array}$ & $\begin{array}{l}0 \\
0 \\
0\end{array}$ & $\begin{array}{r}527991 \\
1105000 \\
874018\end{array}$ \\
\hline 2507009 & 0 & 2507009 & 0.00 & 0 & 2507009 & 0 & 0 & 2507009 \\
\hline & 78,860 & & & 113,995 & & & $1,800,106$ & \\
\hline & & & 1.99 & & 84 & 15 & & 20,488 \\
\hline
\end{tabular}


WHC-SD-SNF-RPT-012, Rev. 0

ICF KAISER HANFORD

WEST INGHOUSE HANFORD COMPANY

JOB NO. E $57991 / 2409$

FILE NO. Z409PAA3
* * IEST - INTERACTIVE ESTIMATING * *

K-BASINS INTEGRATED HATER SYSTEM PRELIMIHARY

DOE_RO3 - ESTIMATE BASIS SHEET $\begin{array}{ll}\text { PAGE } & 5 \quad \text { OF } 15 \\ \text { DATE } & 07 / 24 / 96 \\ \text { BY } & \text { JPM SMF DKH LGH }\end{array}$

1. DOCUMENTS AND DRAHINGS

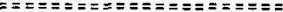

DOCUMENTS: $\quad$ DRAFT FOC DATED JUNE 1996 WHC-SD-SNF-FDC-003, REV O

ORAWINGS: $\quad$ PRELIMINARY DRAWINGS H-1-81986,87 H-1-82014, 15, 16, 17,18

2. MATERIAL PRICES

$===\pi========$

UNIT COSTS REPRESENT CURREHT PRICES FOR SPECIFIED MATERIAL. VENDOR INFORMATION HAS OBTAINED FOR THE FOLLOHING ITEMS: SEE ATTACHED VENDOR QUOTES

3. LABOR RATES

A.) ICF-KH HOURLY RATES ARE BASED ON THE 1996 FISCAL YEAR BUDGET LIQUIDATION RATES AS ISSUED BY KEH FINANCE

(EFFECTIVE 3-08-96). SEE ALSO THE FY 1996 PLANHING RATES * (REPORT BGHB7012).

B.) HHC HOURLY RATES ARE BASED UPON THE FY 1996 PLANNING RATES - (REPORT BGHB2OO1).

C.) IRM HOURLY RATES ARE BASED UPON THE FY 1996 PLANNING RATES - (REPORT BGHBTOO8)

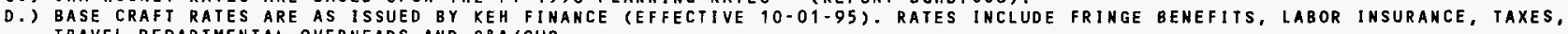
TRAVEL, DEPARTMENTAL OVERHEADS AND G\&A/SHS.

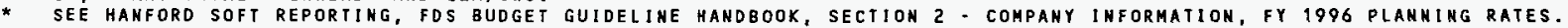

4. GENERAL REQUIREMENTS / TECHHICAL SERVICES/OVERHEADS

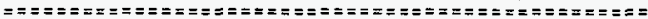

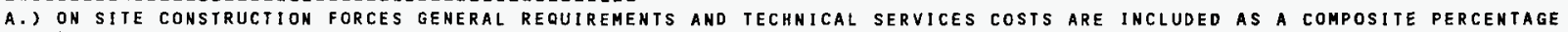

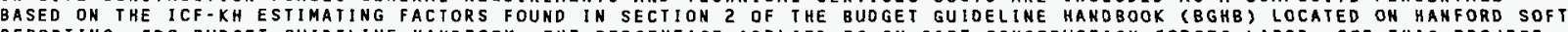

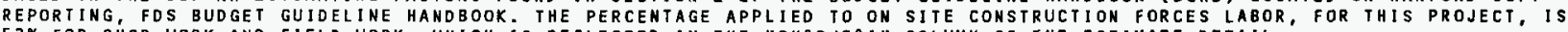
$52 \%$ FOR SHOP WORK AND FIELD HORK, WHICH IS REFLECTED IN THE "OH\&P/B\&I" COLUMN OF THE ESTIMATE DETAIL.

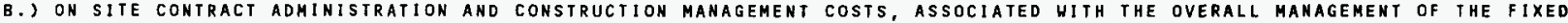
PRICE CONTRACTS, ARE INCLUDED AS A COMPOSITE PERCENTAGE AND LUMP SUM ALLOHANCE (FOR BID PACKAGE PREP) BASED ON THE ESTIMATING FACIORIBILLIMG SCHEDULE THE TOTAL COMPOSITE PERCENTAGE AND LUMP SUM ALLOUANCE ARE APPLIED AGAINST THE TOTAL FIXED PRICE CONTRACT AMOUHT WHICH IS REFLECTED ON THE KEH SUMMARY REPORT DOE ROT, INCLUDED HITH THIS ESTIMATE. (FINAL ESTIMATES MAY BE PARTIALLY MANLOADED AND INCLUDED WITHIN THE ESTIMATE DETAIL)

C.) FIXED PRICE CONTRACTOR OVERHEAD, PROFIT, BOND AND INSURANCE COSTS HAVE BEEN APPLIED AT THE FOLLOHING PERCENTAGES AND ARE REFLECTED IN THE "OH\&P/B\&I" COLUMN OF THE ESTIMATE DETAIL:

LABOR - 25\% MATERIAL $-25 \%$ EQUIPMENT USAGE - 10\% EQUIPMENT-10\% SUBCONTRACTS - 10\%

5. ESCALATION

$=\Sigma===\Sigma===$

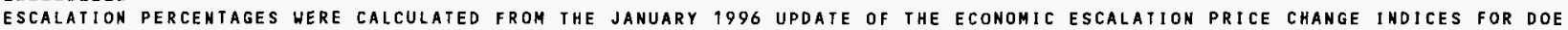
CONSTRUCTION PROJECTS AS PUBLISHED BY THE "OFFICE OF INFRASTRUCTURE ACOUISIIION" FM-5O.

6. ROUNDING

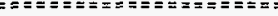

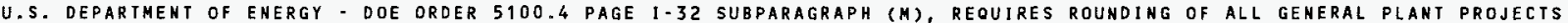
(GPPIS) AND LINE ITEM (LI) COST ESTIMATES. REFERENCE: DOE 5100.4, FIGURE I-11, DATED 10-31-84. 
ICF KAISER HANFORD

WESTINGHOUSE HANFORD COMPANY

JOB NO. EST991/2409

FILE NO. 2409 PAA3

7. REMARKS

$=====$

A.) ESTIMATE DOES NOT IHCLUDE ENCAPSULATIOH OR REMOVAL OF ASEESTOS HATERIALS.

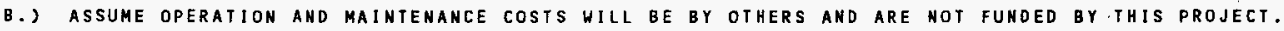

C.) ASSUME PENETRATION OF BASIN CONCRETE WALLS OR FLOORS WILL NOT BE REQUiRED.

D.) ASSUME EXISTING BUILOING NALLS ABOVE GRADE ARE NOT OF CONCRETE CONSTRUCTION.

E.) ASSUME LESS THAN ONE (1) MR RAD DOSE IN $105 K$ BH BSIN AND RADIAIION DOSES (15) FIFTEEN MR OR LESS AT 105KE BASIN.

F.) HEPA FILTER SYSTEMS HILL BE FOR VESSEL ONLY IN ANNEX BUILOING AND WEASEL PIT ONLY IN 1OS KE BASIN.

G). ASSUHE TITLE III "EhgINEERING INSPECTION" HILL be SHARED BETHEen OFFSITE ENG AND OHSITE ENGINEER.

H.) RELOCATE EXISTING MMI TO ROOM 77 OF KE-BASIN OPERATIONAL FACILITIES

1.) INSTALL MMI AND PLC IN ROOM \#5 OF KH-BASIN OPERATIONAL FACILITIES

J.) PIPE SUPPORT ALLOHANCES WERE PATTERNED FROM SUPPORTS FOUND ON DRAHIMG H-1-34693

K.) PROCUREMENT OF SLUDGE AND DEBRIS REMOVAL PUMPS ARE NOT FUNDED BY THIS PROJECT.

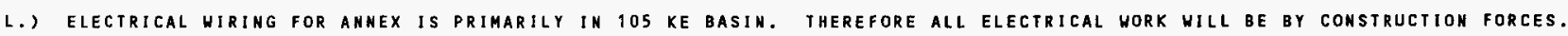

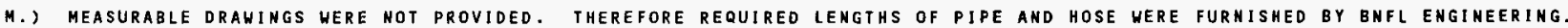

N.) AS-BUILOING HILL BE BY PROJECT DRAWINGS HITHOUT VARIATIONS.

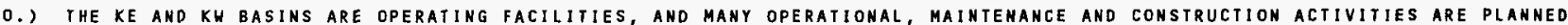

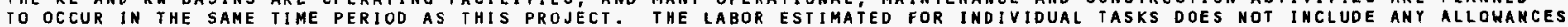
FOR DOHN-TIME THAT MIGHT OCCUR FROM INTERFERENCES HITH THESE OTHER ACTIVITIES.

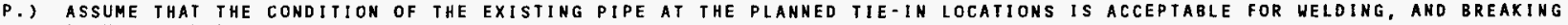
FLANGED CONNECTIONS.

Q.) ASSUME ALL RADIOACTIVE WASTE generATED BY THIS PROJECT CAH BE Disposed Of AS LOH-LEVEL HASTE.

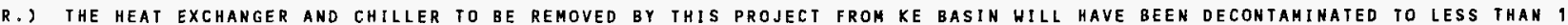
$M R / H R$ BY OTHERS

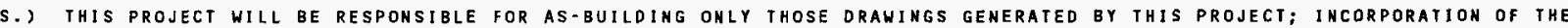
CHANGES INTO EXISTING DRAHINGS FOR KE AND KW IS NOT INCLUDED. 
WHC-SD-SNF-RPT-012, Rev. 0

ICF KAISER HANFORD
WESTINGHOUSE HANFORD COMPANY
JOB NO. EST991/Z409

FILE NO, Z409PAA3

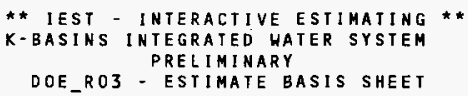

PAGE 7 OF 15

DATE $07 / 24 / 96$

BY JPM SMF DKH LGH

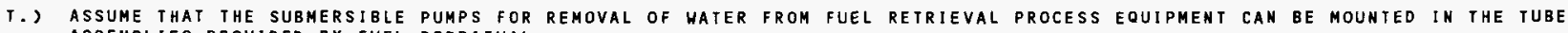
ASSEMBLIES PROVIDED BY FUEL RETRIEVAL,

U.) ASSUME THAT SECURITY ESCORTS WILL NOT BE REQUIRED FOR CONSTRUCTION PERSONNEL IN THE KE AND KW BASINS.

V.) ASSUMe that electrical poner SUPPLies avallable in the basins hill be sufficient for the needs of this project.

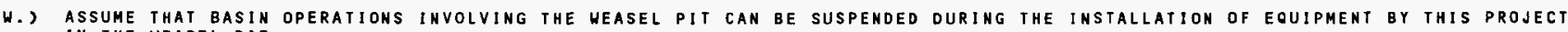
IN THE HEASEL PIT.

X.) ENGINEERING INSPECTION (WBS 1.2) PERCENTAGE IS A COMPILATION OF THE FOLLOHING COSTS

$\begin{array}{ll}\text { ESTIMATE SUBTOTAL (DIRECT CONSTRUCIION COSTS) } & 5,018,115 \\ \text { LESS BURNOUT DIRECT COSIS, ESTIMATE DIRECT } & \$ 324,191 \\ \text { LESS BURIAL EXPENSES. ESTIMATE DIRECT } & \$ 94,380\end{array}$

Y.) OTHER PROJECT COSTS (OPC'S) INCLUDE ESCALATION AND CONTINGENCY IN THEIR BASE hUMBERS.

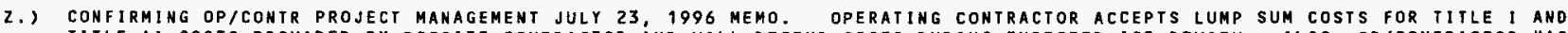

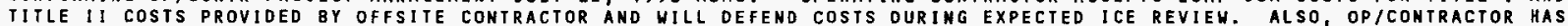
AUTHORED CONTINGENCY INFORMATION (REF DOE_6 REPORT) FOR IITLE I, TITLE II CONTINGENCY ANALYSIS.

Aa.) OFFSITE ENGINEERING DEFINITIVE DESIGN WORK PLAN IS ATTACHED FOR REVIEH. 
WHC-SD-SNF-RPT-012, Rev. 0

ICF KAISER HANFORD

WESTINGHOUSE HANFORD COMPANY

JOB NO. E57991/2409

FILE NO. Z409PAA3

$\cos T$

CODE / HBS

DESCRIPT $10 \mathrm{OH}$

010 TITLE I DESIGN

112100 TITLE I DEF/DESIGH - OFFSITE A/E

TOTAL 010 TITLE I DESIGN

020 TITLE II DESIGN

112210 TIILE II DEF/DESIGN - OFFITE A/E 1600000

TOTAL 020 IITLE II DESIGN

1600000

* IEST - INTERACTIVE ESTIMATING **

K-BASINS INTEGRATED WATER SYSTEM

PRELININARY

DOE_RO4 - COST CODE ACCOUNT SUMMARY

SUBTOTAL INDIRECTS TOTAL

$==\boldsymbol{=}=\boldsymbol{=}=$

$== \pm==+=$

240000

$0 \quad 240000$

240000

$0 \quad 1600000$

o 1600000
PAEE 8 OF 15

DATE $07 / 24 / 96 \quad 11: 28: 35$

BY JPM SMF DKH LGH

ESTIMATE ONSITE SUB ESCALATION SUB CONTINGENCY TOTAL

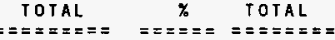

DOLLARS

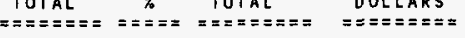

0.00

0.00

240000

240000

0

240000

0

140000

0

$0 \quad 240000$

30 TITLE III CONST. ENG/INSPECIION

122000 ENGINEERING/INSPECTION-OFFSITE A/E

413959

413959

0.00

1600000

0.00

1600000

160000

1760000

o

160000

1760000

TOTAL 030 TITLE III CONST. ENG/INSPECT

7079

421038

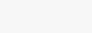

20

\title{
060 PROJECT MANAGEMENT
}

\section{PROJECT MANAGEMENT}

TOTAL 060 PROJECT MANAGERENT

811000

811000

$413959 \quad 1.71$

7079

$421038 \quad 30$

126311

547349

4

(2)

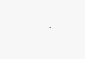

$\begin{array}{rl}816434 & 1 \\ 816434 & 10 \\ & \\ 158439 & 1 \\ 95063 & 2 \\ 598264 & 30 \\ 77510 & 30 \\ 48267 & 25 \\ 72754 & 20 \\ 6282 & 30 \\ 180297 & 28 \\ 68874 & 20 \\ 64027 & 20\end{array}$

0.67
0.67
5434
5434

811000

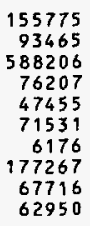
1. 71
1.71
1.71
1.71
1.71
1.71
1.71
1.72
1. 71
1.71
1.71
2664
1598
10058
1303
812
1223
1223
1158
1076

321200
321201

54347
50522

41018
13369
12428

62950

\begin{abstract}
1.71
\end{abstract}

\begin{abstract}
64027
\end{abstract}

$\begin{array}{lr}15 & 23766 \\ 20 & 19013 \\ 30 & 179479 \\ 30 & 23253 \\ 25 & 12067 \\ 20 & 14551 \\ 30 & 1884 \\ 28 & 50782 \\ 20 & 13775 \\ 20 & 12805\end{array}$
182205
114076
777744
100763
60333
87305
8166
231079
82649
76832


WHC-SD-SNF-RPT-012, Rev. 0

I CF KAISER HANFORD

WESTINGHOUSE HANFORD COMPANY

JOB NO. E $57991 / 2409$

FILE NO. 2409 PAA3

$\cos T$

CODE/HBS

DESCR I PTION

321204 COMP/AIR AND SAFETY SHOWER

321205 HVAC \& HEPA FILFRATION

TOTAL 501 BUILOINGS

550 OTHER STRUCTURES

310191 NEW RAD STORAGE PAD

TOTAL 550 OTHER STRUCTURES

700 SPECIAL EQUIPIPROCESS SYSTEMS

310101

310102

310103

310104

310106

310107

310108

310109

310110

310111

310112

310113

310115

310116

310118

310119

310180

310190

310300

310307

310308

310309

310390

310311

DER BAY MODIFICATIONS

PRIMARY CLEANING STATION PIPING

DOUN ORAFT TABLE PIPING

MISC DRAIN VENT \& RETURM TO BASIM

DEBRIS REMOVAL PIPING

WEASEL PIT PUMP \& PIPING

HYOROCYCLONES \& PIPING

FILTER BACKWASH PIPING

SW LOADOUT PIPING

DEMINERALI I ZED WATER

WEASEL PIT MODIFICATIONS

B/W FLTRS SUPPLY AND RETURH

POWER WIRING KE

INSTRUMENTATION KE

CHILLER HEAT EXCHANGER DEMOLITION

PAINT AND LABEL PIPING

BURHOUT

SUPPORT REQUIREMENTS

MOCK - UP FACILITIES \& TRAINING

MEU RECIRC PUMPS

TRANSFER BAY MODIFICATIONS

DELID STATION

PRIMARY CLEAN STATION

DOWN DRAFT TABLE \& DRY CANISTER STA

DEBR IS REMOVAL

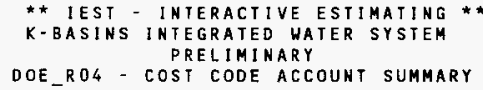

* * IEST - INTERACTIVE ESTIMATING * *

K-BASINS INTEGRATED WATER SYSTEM

PRELIMINARY

DOE R04 - COST CODE ACCOUNT SUMMARY

PAGE 9 OF 15

DATE $07 / 24 / 96 \quad 11: 28: 35$

BY JPM SMF DKH LGH

\begin{tabular}{|c|c|c|c|c|c|c|c|c|}
\hline $\begin{array}{l}\text { ESTIMATE } \\
\text { SUB TOTAL } \\
=======\end{array}$ & $\begin{array}{l}\text { ONSITE } \\
\text { INDIRECTS } \\
=======\end{array}$ & $\begin{array}{c}\text { SUB } \\
\text { TOTAL } \\
=========\end{array}$ & $===\stackrel{\text { ESCA }}{\%}=$ & $\begin{array}{l}\text { ILATION } \\
\text { TOTAL } \\
======\end{array}$ & $\begin{array}{c}\text { SUB } \\
\text { IOTAL } \\
========\end{array}$ & $\begin{array}{c}\text { CONT } \\
\mathbf{x} \\
===\end{array}$ & $\begin{array}{l}\text { INGENCY } \\
\text { TOIAL } \\
=========\end{array}$ & $\begin{array}{c}\text { TOTAL } \\
\text { DOLLARS } \\
=========\end{array}$ \\
\hline $\begin{array}{l}26963 \\
22000\end{array}$ & $\begin{array}{l}6633 \\
5412\end{array}$ & $\begin{array}{l}33596 \\
27412\end{array}$ & $\begin{array}{l}1.71 \\
1.71\end{array}$ & $\begin{array}{l}574 \\
469\end{array}$ & $\begin{array}{l}34170 \\
27881\end{array}$ & $\begin{array}{l}20 \\
20\end{array}$ & $\begin{array}{l}6834 \\
5576\end{array}$ & $\begin{array}{l}41004 \\
33457\end{array}$ \\
\hline 1328896 & 78860 & 1407756 & 1.71 & 24072 & 1431828 & 25 & 363785 & 1795613 \\
\hline
\end{tabular}

$\begin{array}{lllllllll}232445 & 0 & 232445 & 1.71 & 3975 & 236420 & 20 & 47283 & 283704 \\ 232445 & 0 & 232445 & 1.71 & 3975 & 236420 & 20 & 47283 & 283704\end{array}$

142236
58296
63935
78391
13890
74923
70579
135964
76996
90556
15422
409110
307131
35410
518604
354751
14416
295355
206056
142236
73989
147851
38065
20290
88882
24044

$\begin{array}{rr}142236 & 1.71 \\ 58296 & 1.71 \\ 63935 & 1.71 \\ 78391 & 1.71 \\ 13890 & 1.71 \\ 74923 & 1.71 \\ 70579 & 1.71 \\ 135964 & 1.71 \\ 76996 & 1.71 \\ 90556 & 1.71 \\ 15422 & 1.71 \\ 409110 & 1.71 \\ 307131 & 1.71 \\ 35410 & 1.71 \\ 518604 & 1.71 \\ 354751 & 1.71 \\ 14416 & 1.71 \\ 295355 & 1.71 \\ 206056 & 1.71 \\ 142236 & 1.71 \\ 73989 & 1.71 \\ 147851 & 1.71 \\ 38065 & 1.71 \\ 20290 & 1.71 \\ 88882 & 1.71 \\ 24044 & 1.71\end{array}$
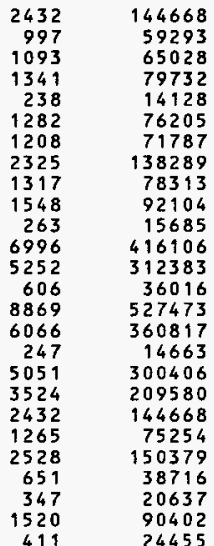

20
23
20
20
27
21
21
20
20
20
20
23
20
25
25
30
20
30
20
20
16
20
20
20
20
20

28934
13563
13005
15946
3822
16168
15212
27658
15662
18421
3137
97271
62477
9004
134029
108245
2933
90122
41916
28934
12321
30075
7743
4127
18081
4891

173602
72856
78034
95678
17950
92373
86998
165946
93975
110526
18823
513377
374860
45019
661500
469062
17595
390527
251495
173602
87575
180455
46459
24764
108483
29346 
WHC-SD-SNF-RPT-012, Rev. 0

ICF KAISER HANFORD

WESTINGHOUSE HANFORD COMPANY

JOB NO. ES7991/Z409

FILE NO. Z409PAA3

$\cos T$

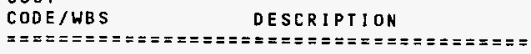

310313 SH LOAD OUT PIT

310314 DEMINERALI ZED WATER

310315 POWER WIRING KW

310316 INSTRUMENTATION KH

310317 PAINT AND LABEL PIPING

310380 BURNOUT

310390 SUPPORT REQUIREMENTS

330000 BURIAL FEES

TOTAL 700 SPECIAL EQUIP/PROCESS SYSTEM

900 OTHER PROJECT COSTS

$\begin{array}{llllll}500096 & \text { FY } 96 & \text { OIHER PROJECT } & \text { COST } \\ 500097 & \text { FY } 97 & \text { OTHER PROJECT } & \text { COST }\end{array}$

$\begin{array}{lllll}500097 & \text { FY } & 97 & \text { OTHER } & \text { PROJECT } \\ 50009 & \text { COST } \\ 50009 & \text { FY } 98 & \text { OTHER PROJECT } & \text { COST }\end{array}$

TOTAL 900 OTHER PROJECT COSTS

PROJECT TOTAL
* * IEST - INTERACTIVE ESTIMATING * *

K-BASINS INTEGRATED HATER SYSTEM

PRELIMINARY

DOE_RO4 - COST CODE ACCOUNT SUMMARY
PAGE 10 OF 15

DATE $07 / 24 / 96 \quad 11: 28: 36$

BY JPM SMF DKH LGH
SUBTOTAL

$====$

27933
11461

13919

441230

8891

28836

170192

94380

4294220

\section{3}

11461

13919

441230

8891

28836

170192

94380

$0 \quad 4294220$

o

1.71

478
196
238
7545
152
493
2910
1614
73435

1.71

1.71

1. 71

1. 71

1.79

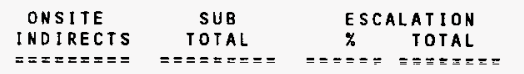

SUB
TOTAL

CONTINGENCY

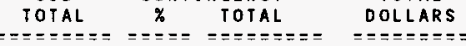

TOTAL

$\begin{array}{llll}1.71 & 478 & 28411 & 20\end{array}$

11657

14157

448775

9043
29329

29329
173102

95994

20

20
25
25

30

20

5682
2331
3539

111809

1809

8799

34620
28708

$4367655 \quad 23$

1021084

34093

13988

17696

560584

10852

38128

207723

124792

5388736
527991
1105000

1105000
874018

2507009
0

2507009

0.00

$\begin{array}{rrr}0 & 527991 & 0.00 \\ 0 & 1105000 & 0.00 \\ 0 & 874018 & 0.00\end{array}$

$\begin{array}{rrr}0 & 527991 & 0 \\ 0 & 1105000 & 0 \\ 0 & 874018 & 0 \\ 0 & 2507009 & 0\end{array}$

527991

874018

2507009

2507009

0

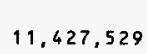

78,860

113,995

$1,800,106$

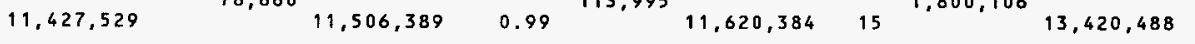


WHC-SD-SNF-RPT-012, Rev. 0

ICF KAISER HANFORD

WESTINGHOUSE HANFORD COMPANY

JOB NO. E57991/2409

FILE NO. Z409PAA3

\section{CSI DESCRIPT I ON}

CONSTRUCTION

$\begin{array}{ll}00 & \text { TECHHICAL SERVICES } \\ 01 & \text { GENERAL REQUIRMENTS } \\ 02 & \text { SITEHORK } \\ 03 & \text { CONCRETE } \\ 05 & \text { METALS } \\ 09 & \text { FINISHES } \\ 10 & \text { SPECIALTIES } \\ 13 & \text { SPECIAL CONSTRUCTION } \\ 14 & \text { CONVEYING SYSIEMS } \\ 15 & \text { MECHANICAL } \\ 16 & \text { ELECTRICAL } \\ 19 & \text { PROJECT MANAGEMENT } \\ 20 & \text { BURNOUT } \\ 90 & \text { OTHER PROJECT COSTS }\end{array}$

TOTAL CONSTRUCTION

\section{PROJECT TOTAL}

* * IEST - INTERACTIVE ESTIMATING * *

K-BASINS INTEGRATED WATER SYSTEM

PRELIMINARY

DOE_ROS - ESIIMATE SUMMARY BY CSI OIVISION
PAGE 11 OF 15

DATE $07 / 24 / 96 \quad 11: 28: 39$

BY JPM SMF DKH LGH

\begin{tabular}{|c|c|c|c|c|c|c|}
\hline $\begin{array}{l}\text { ESTIMATE } \\
\text { SUBTOTAL } \\
======\end{array}$ & $\begin{array}{l}\text { ONSITE } \\
\text { INDIRECIS } \\
=======\end{array}$ & $\begin{array}{c}\text { SUB } \\
\text { IOTAL } \\
=======\end{array}$ & $==\begin{array}{c}\text { ESCALATION } \\
\% \text { TOIAL } \\
=========\end{array}$ & $\begin{array}{c}\text { SUB } \\
\text { IOTAL } \\
=======\end{array}$ & $\begin{array}{cc}\text { CONTINGENCY } \\
\psi_{0} \text { TOTAL } \\
===========\end{array}$ & $\begin{array}{c}\text { TOTAL } \\
\text { DOLLARS } \\
========\end{array}$ \\
\hline
\end{tabular}

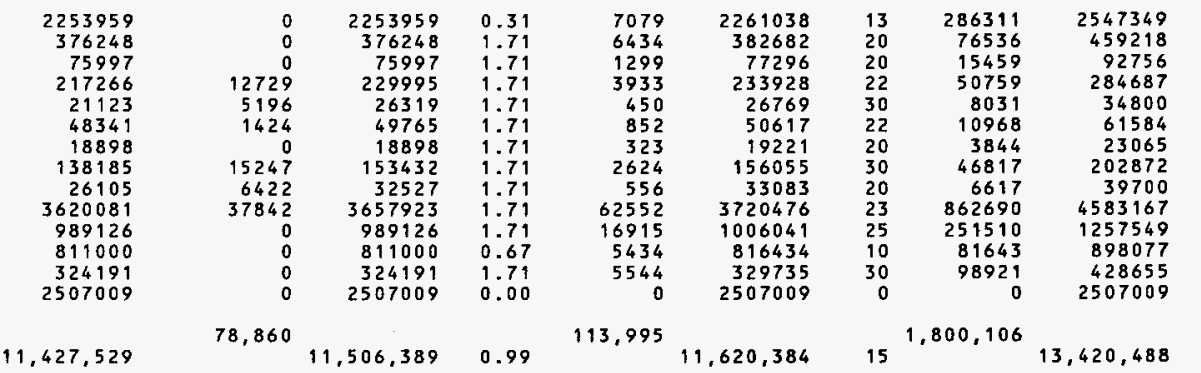

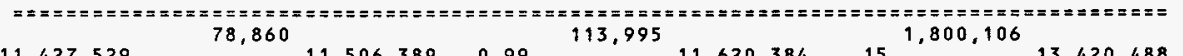

$11,427,529$

$11,506,389$

0.99

$11,620,384$

15

$13,420,488$ 
WHC-SO-SNF-RPT-012, Rev. 0

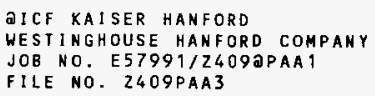

PAGE 12 OF 15

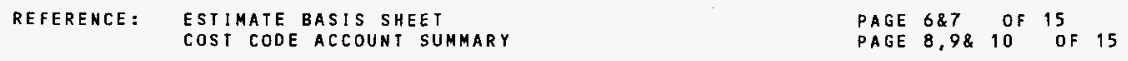

THE U.S. DEPARTMENT OF ENEREY - RICHLAMD ORDER 5700.3 "COST ESTIMATIHG, ANALYSIS AND STANDARDIZATIOA!

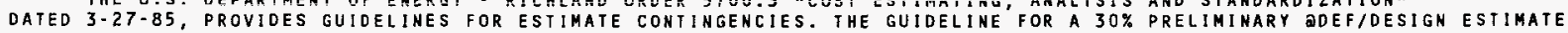
SHOULD HAVE AN OVERALL RANGE OF $10 \%$ TO $20 \%$.

CONTINGENCY IS EVALUATED AT THE THIRD COST CODE LEVEL AND SUMMARIZED AT THE PRIMARY AND SECONDARY COST CODE LEVEL OF THE DETAILED COST ESTIMATE.

WBS 1.1

ENGINEERING

COST CODE 010

$\cos T \operatorname{CODE} 020$

WBS $1.2^{\cos T \operatorname{code} 030}$

HBS $4.0^{\operatorname{COST} \operatorname{CODE} 060}$

CONSTRUCTION

HBS 2.2

WBS 3.1

WBS 3.2
(TILE I DEFINITIVE DESIGN)

NO CONTINGENCY HAS APPLIED AS TITLE I IS COMPLETE

A 10\% CONTINGENCY HAS BEEN APPLIEO TO TITLE II TO ALLOW FOR GROHTH DUE TO DEMOHSTRATION IESTING FOR BACK WASHABLE FILTER SKID INTENDED TO BE USED IN KE WILL NOT BE PERFORMED UNTIL AFTER MUCH OF THE PROCESS DESIGN IS CONPLETED. IF THIS FILTER DOES NOT FUNCTION AS PLANED, SIGNIFICANT RE-DESIGN WILL BE REQUIRED.

A 30\% CONTINGENCY HAS APPLIED TO IITLE III DUE THE CONOITION AND ACCURACY OF AS-BUILT DRALINGS FOR K-BASINS IS QUESTIONABLE, AND OTHER PROJECTS ARE PLANIING ON MODIFYING THE BASIN CONFIGURATION AHD DRAWINGS AT THE SAME TIME AS THIS PROJECT. THIS COMPOUNDS THE COORDIHATION REQUIREO BETHEEN THIS AND OTHER PROJECTS, AND MAKES IT VERY DIFFICULT TO DETERMING THE DEGREE OF AS-BUILDING AND WALK-DOWN THAT HILL BE REQUIRED BY THIS PROJECT.

(PROJECT MANAGEMENT)

A $10 \%$ CONTINGENCY WAS APPLIED DUE TO UNCERTAINTIES IN PERMITTING ACTIVITIES AND SAFETY ANALYSIS MULTIPLE TIER REVIEHS OF PERMITTING AND SAFETY DOCUMENTS, DEVELOPMENT OF DOCUMENTATION TO SUPPORT KEY DECISIONS.

AVERAGE ENGINEERING CONTINGENCY $13 x$

(BUILDINGS) QUOTATIOHS. HOHEVER THE AGGRESSIVE SCHEDULE OF PROJECT PROMPTS ADOITION OF CONTIHGENCY TO ALLOH SUPPLIERS TO MEET SHORT DELIVERY TIME.

A $20 \&$ CONTINGENCY HAS BEEN APPLIED TO PROCUREMENT DUE TO PRESENT LEVEL OF PRELIMIHARY DETAILS OF IXM EQUIPMENT ANO A $30 \%$ CONTINGENCY HAS BEEN APPLIED TO BACKWASH FILTERS DUE TO SINGLE VENOER QUOTE.

A 20 TO $25 \%$ CONTINGENCY HAS INCORPORATED INTO MECHANICAL AND ELECIRICAL BUILDING WORK ACTIVITIES OUE TO UNKNOWN MATERIAL AND LABOR AT ELECTRICAL AND MECHANICAL TIE INS AND DIFFICULTY HORKING IN A OPERATING FACILITY.

A 20\% CONTINGENCY HAS BEEN APPLIED TO NEN ANNEX CONSTRUCIION DUE TO POSSIBLE CHANGES IN BUILDING FOOTPRINT AND SIZE, ALSO PRELIMINARY DESIGN PLACES ANNEX BUILDING ON EXISTING KNOWN CONTAMINATED CONCRETE STORAGE PAD AND THIS COULD REQUIRE SHP REQUIREMENTS. 
ICF KAISER HANFORD WEST INGHOUSE HANFORD COMPANY JOB NO. E57991/2409 FILE NO. Z409PAA3
* * IEST - INTERACTIVE ESTIMATING * *

K-BASINS INIEGRATED WATER SYSTEM

PRELIMINARY

DOE_ROG - CONTINGENCY AMALYSIS BASIS SHEET
PAGE 13 OF 15

DATE $07 / 24 / 96$

BY JPM SHF DKH LGH
COHSTRUCTION

WB 3 .

COST CODE 700

(SPECIAL EQUIP/PROCESS SYSTEMS)

A $20 \%$ TO $30 \%$ CONTINGENCY HAS BEEN APPLIED TO CONSTRUCTION ACTIVITIES DUE TO THE

CONCERNS OF ENCOUNTERING UNKNOUN LEVELS OF CONTAMINATED MATERIALS, HORKING IH PROCESS

FACILITY, POSSIBLE OTHER CONSTRUCTION PROJECTS RUNHING CONCURRENTLY MAY CAUSE THIS PROJECT

TO GO ON SHP AND FULL MASK AND TO CONCERHS MADE FDR QUANTITY OF CONTAMIMATED MATERIALS

IHAT HILL REQUIRE BURIAL.

AVERAGE CONSTRUCTION CONTINGENCY

$23 \%$

AVERAGE PROJECT CONTINGENCY

$15 x$ 
WHC-SD-SNF-RPT-012, Rev. 0

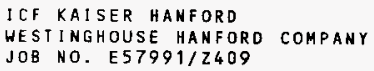

** IEST - INTERACTIVE ESIIMATING * *

K-BASINS INTEGRATED WATER SYSTEM

$$
\text { PRELIMINARY }
$$

DOE_ROT - ONSITE INDIRECT COSTS BY WBS
PAGE 14 OF 15
DATE $07 / 24 / 9611: 28: 43$
BY JPM SMF DKH LGH

OTHE R

INDIRECTS

PREP.

SUBTOTAL

CONTRACT ADMINISTRATION

$==\stackrel{\mathscr{X}}{=}=$ TOTAL

0.00

0.00

0.00

0.00
0.00

0.00
0.00

0.00

0.00

0.00

0.00

0.00

0.00

0.00

0.00

0.00

0.00

0.00

0.00

0.00

0.00

0.00

0.00

0.00

0.00

0.00

0.00

0.00

0.00

0.00

0.00
0.00

0.00

0.00

0.00
0.00

0.00

0.00

0.00

0.00

0.00

0.00

0.00

0.00

0.00

0.00

0.00

0.00

$=======$

$== \pm===$

$==\boldsymbol{=}=\mathbf{=}=$
$1146 ?$

441230

2883

170192
TOTAL I NDIRECTS
$========$

310315 POWER WIRIHE

310390 SUPPORT REQUIREMENTS 
WHC-SD-SNF-RPT-012, Rev. 0

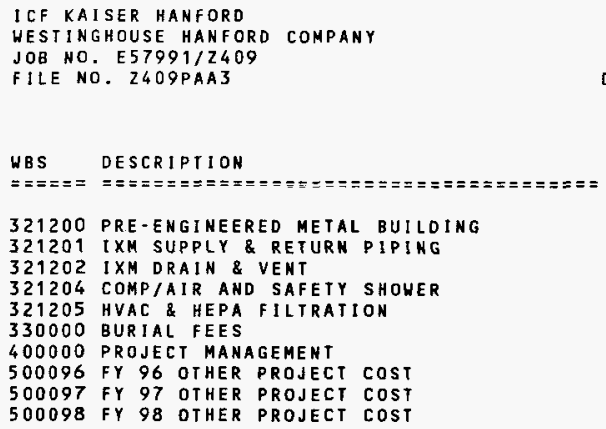

PROJECT TOTAL
* \# IEST - INIERACTIVE ESTIMATING * *

K-BASINS INTEGRATED WATER SYSTEM PRELIMINARY

DOE_ROT - ONSITE INDIRECT COSTS BY WBS

\section{EST IMATE \\ SUBTOTAL \\ CONTRACT ADMINISTRATION$$
\text { TOTAL }
$$

$== \pm= \pm=$

$\%$

$=\equiv=\equiv==$

136249
54347

54347
50522

50522
26963

22000

94380

811000

527991

1105000

874018

$\begin{array}{rr}24.60 & 33518 \\ 24.60 & 13369 \\ 24.60 & 12428 \\ 24.60 & 6633 \\ 24.60 & 5412 \\ 0.00 & 0 \\ 0.00 & 0 \\ 0.00 & 0 \\ 0.00 & 0 \\ 0.00 & 0\end{array}$

PAGE 15 OF 15

PATE $07 / 24 / 96 \quad 11: 28: 43$

BY JPH SMF DKH LGH
$= \pm=\pi=====$
$11,427,529$
OTHER

INDIRECTS

$======$

$$
\begin{aligned}
& 0 \\
& 0 \\
& 0 \\
& 0 \\
& 0 \\
& 0 \\
& 0 \\
& 0 \\
& 0 \\
& 0
\end{aligned}
$$

TOTAL I ND I RECTS

41018 13369 12428 6633 5412 
WHC-SD-SNF-RPT-012, Rev. 0

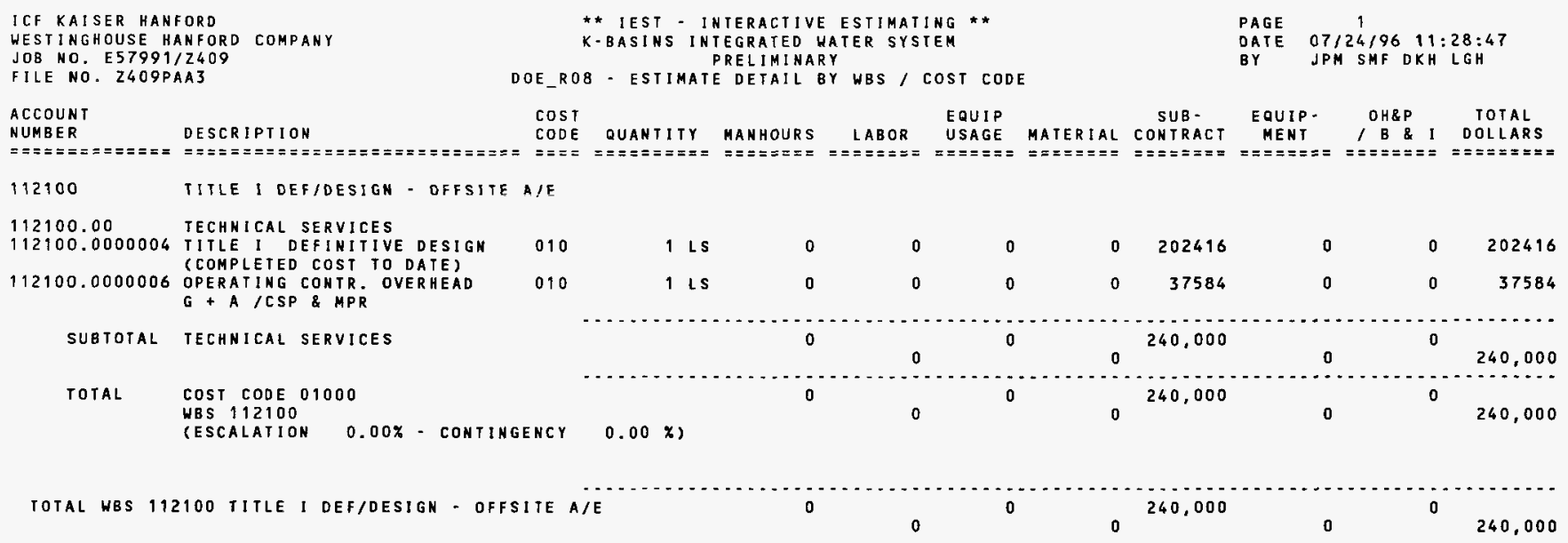


WHC-SD-SNF-RPT-012, Rev. 0

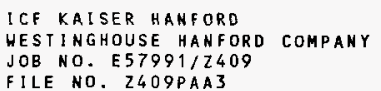

* * IEST - INTERACTIVE ESTIMATING * *

K-BASINS INTEGRATED HATER SYSTEM

$$
\text { PRELIMINARY }
$$

DOE_ROB - ESTIMATE DETAIL BY HBS / COST CODE
PAGE

DATE $07 / 24 / 96 \quad 11: 28: 48$

JPM SMF DKH LGH

ACCOUNT
NUMBER DESCRIPTIOH

112210

$\cos$

CODE QUAMTITY MANHOURS EQUIP

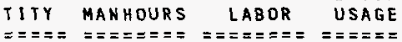
$==+== \pm===$ USAGE
$=====$ MAIERIAL COH
$======$ SUB -
CONTRACT \begin{tabular}{ccc} 
EQUIP- OH\&P & TOTAL \\
MENT & \multicolumn{1}{c}{ B \& I DOLLARS }
\end{tabular} 112210.00 TECHNICAL SERVICES 112210.0000004 PROJECT MANAGEMENT 112210.0000006 PROJECT CONTROL 112210.0000008 QUALITY ASSURAMCE 112210.0000010 SAFETY

112210.0000012 ENGI HEER ING MANAGEMENT

112210.0000014 PROCESS ENGINEERING

$112210.0000016 \mathrm{CIVIL/STRUCTURAL} \mathrm{ENGINEERING}$ 112210.0000018 MECHANICAL ENGINEERING

112210.0000020 HVAC

112210.0000022 ELECIRICAL ENGINEER ING $020 \quad 1$ LS

112210.0000024 I \& ENGINEERING $020 \quad 1 \mathrm{LS}$ G $A$ A / CSP \& MPR

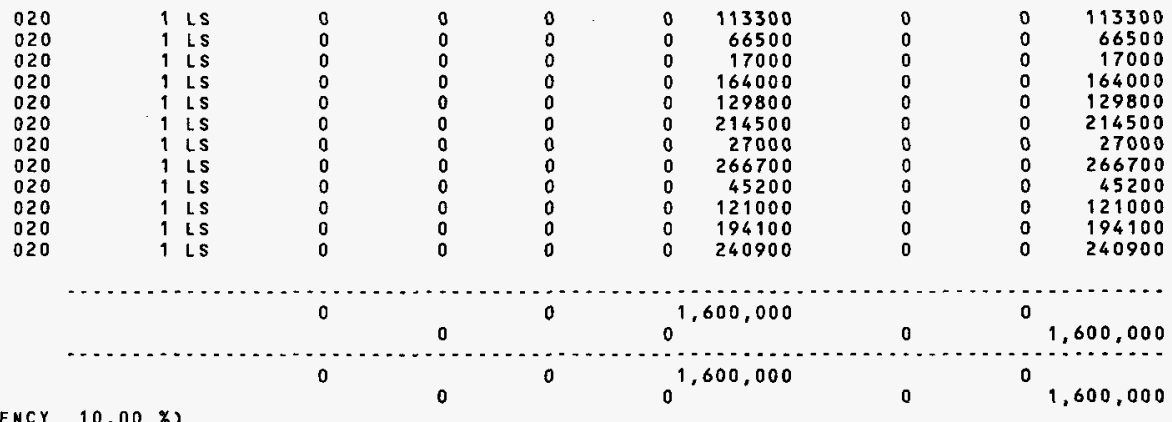

SUBTOTAL TECHNICAL SERVICES

TOTAL

COST CODE 02000

HBS 112210 (ESCALATION

$0.00 \%$ - CONTINGENCY $10.00 \%$
0 $==\mathbf{=}=$ 
WHC-SD-SNF-RPT-012, Rev. 0

ICF KAISER HANFORO

WESTINGHOUSE HANFORD COMPANY

JOB NO. E57991/2409

FILE NO. 2409PAA3

ACCOUNT

NUMBER

DESCRIPTION

ENGINEERING/INSPECTION-OFFSITE A/E

122000

TECHNICAL SERVICES

122000.00

122000.0000004 ENGINEERING INSPECTION $9 \%$ OF $\$ 4,599,544$

030

* * IEST - INTERACTIVE ESTIMATING *

K-BASINS INTEGRATEO WATER SYSTEM

PREL IMINARY

DOE_ROB - ESTIMATE DETAIL BY WBS / COST CODE

COST
CODE QUANTITY MANHOURS LABOR UQUIP

SUB-
MATERIAL CONTRACT

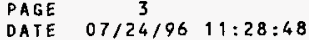

BY JPM SMF DKH LGH

EOUIP-

MENT

B \& TOTAL (TOTAL DIRECT CONSTR LESS BURNOUT, LESS BURIAL FEES)

SUBTOTAL TECHNICAL SERVICES

TOTAL COST CODE 03000

HBS 122000

CESCALATION

$1.71 \%$ - CONTINGENCY $30.00 \%$

$1 \mathrm{LS}$

0

0

413959

0

0

413959

$\ldots \ldots \ldots \ldots$

0

0

413,959

0

0

13.959

(1)

0

.

TOTAL WBS 122000 ENGINEERING/INSPECTION-OFFSITE A/E
0
0
413,959

0

413,959 
WHC-SD-SNF-RPT-012, Rev. 0

I CF KAISER HANFORD

WESTINGHOUSE HANFORD COMPANY

JOB NO. E57991/2409

FILE NO. Z409PAA3

ACCOUNT

NUMBER

DESCRIPI ION

* * IEST - INTERACTIVE ESTIMATING *

K-BASINS INTEGRATED HATER SYSTEM

$$
\text { PRELIMINARY }
$$

DOE_ROB - ESTIMATE DETAIL BY WBS / COST CODE

COST EQUIP

CODE QUANTITY MANHOURS LABOR USAGE

SUB -
MATERIAL CONTRACT

SUB -
NTACT

PAGE

DATE $07 / 24 / 96 \quad 11: 28: 48$

BY JPM SMF DKH LGH

220001

K-EAST IXM PROCUREMENT

220001.15 MECHANICAL

220001.1500004 IXM MIXED BED COLUHA

220001.1500006 IXM AMION COLUMH

220001.1500008 IXM SPARE COLUMN

$\begin{array}{ll}\text { SUBTOTAL } & \text { MECHAHICAL, } \\ & \text { SALES TAX } 8.00 \% \\ & \text { HAREHOUSING } 16.62 \%\end{array}$

TOTAL COST CODE 50115

HBS 220001

$1.71 \times$ - CONTIHGENCY $15.00 \%$

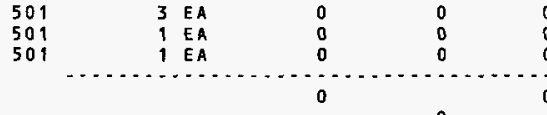

155,775
0

0

125,000 10000 20775

0

75000

25000 25000

0
EQUIP- OH\&P TOTAL MENT $I$ B \& I DOLLARS

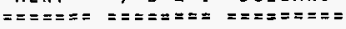

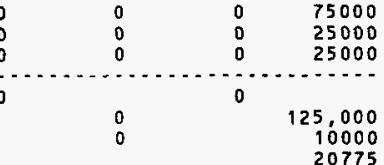

0
0

TOTAL WBS 220001 K-EAST IXM PROCUREMENT

155,775

155,775 
WHC-SD-SNF-RPT-012, Rev. 0

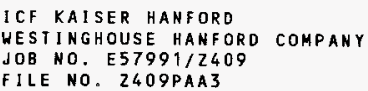

\section{ACCOUNT}

NUMBER

220002

220002.15

220002.1500004 IXM MIXED BED COLUMNS

SUBTOTAL MECHANICAL

SALES TAX $8.00 \%$ HAREHOUSING $16.62 \%$

TOTAL

COST CODE 50115

$1.74 X-$ CONTINGENCY

50
* * IEST - INTERACTIVE ESTIMATING * *

K-BASINS INTEGRATED HATER SYSTEM

PRELIMINARY

DOE_ROB - ESTIMATE DETAIL BY WBS / COST CODE $\begin{array}{lll}\text { PAGE } & 5 \\ \text { DATE } & 07 / 24 / 96 \quad 11: 28: 48\end{array}$

BY JPM SMF DKH LGH

\begin{tabular}{l} 
COST \\
CODE QUANTITY MANHOURS LABOR EUB- \\
\hline
\end{tabular}

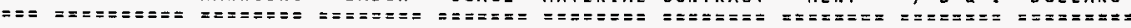

TOTAL WBS 220002 K-HEST IXM PROCUREMENT

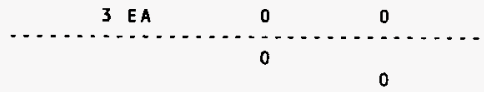

0

$0 \quad 0$

75,000

6000
12465

o

0

$20.00 x)$

93,465

$0 \quad 75,000$
6000

0

0

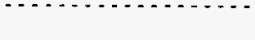

0

0

93,465

0

93,465

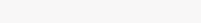


WHC-SD-SNF-RPT-012, Rev. 0

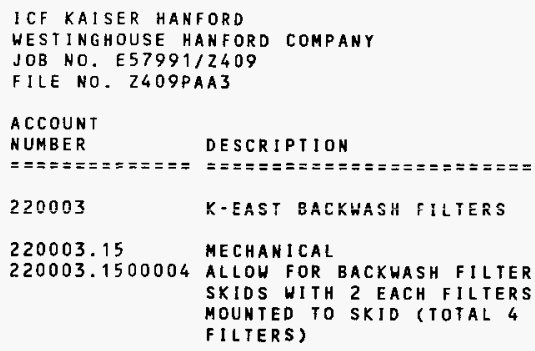
50
* * IESI - INIERACTIVE ESTIMATING * *

K-BASINS INTEGRATED HATER SYSTEM PRELIMINARY DOE_ROB - ESTIMATE DETAIL BY WBS / COST CODE

COST SQUIP SUB- EQUIP- OH\&P TOTAL

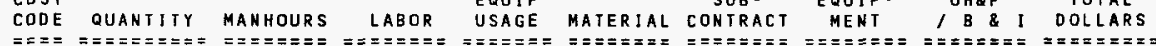

\section{PAGE 6 DATE $07 / 24 / 96 \quad 11: 28: 48$} 2 EA $\mathbf{0}$ 0 0 472000 0 0 o 472000

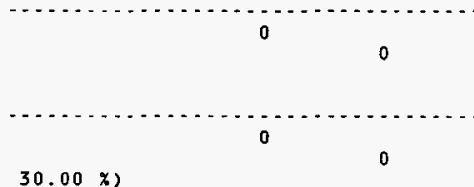

0 472,000

0

37760
78446

0 78446

0 588,206 0 .... 472,000
37760 78446 o -.......
0 o

0

0

588,206

TOTAL WBS 220003 K-EAST BACKHASH FILTERS 
WHC-SD-SNF-RPT-012, Rev. 0

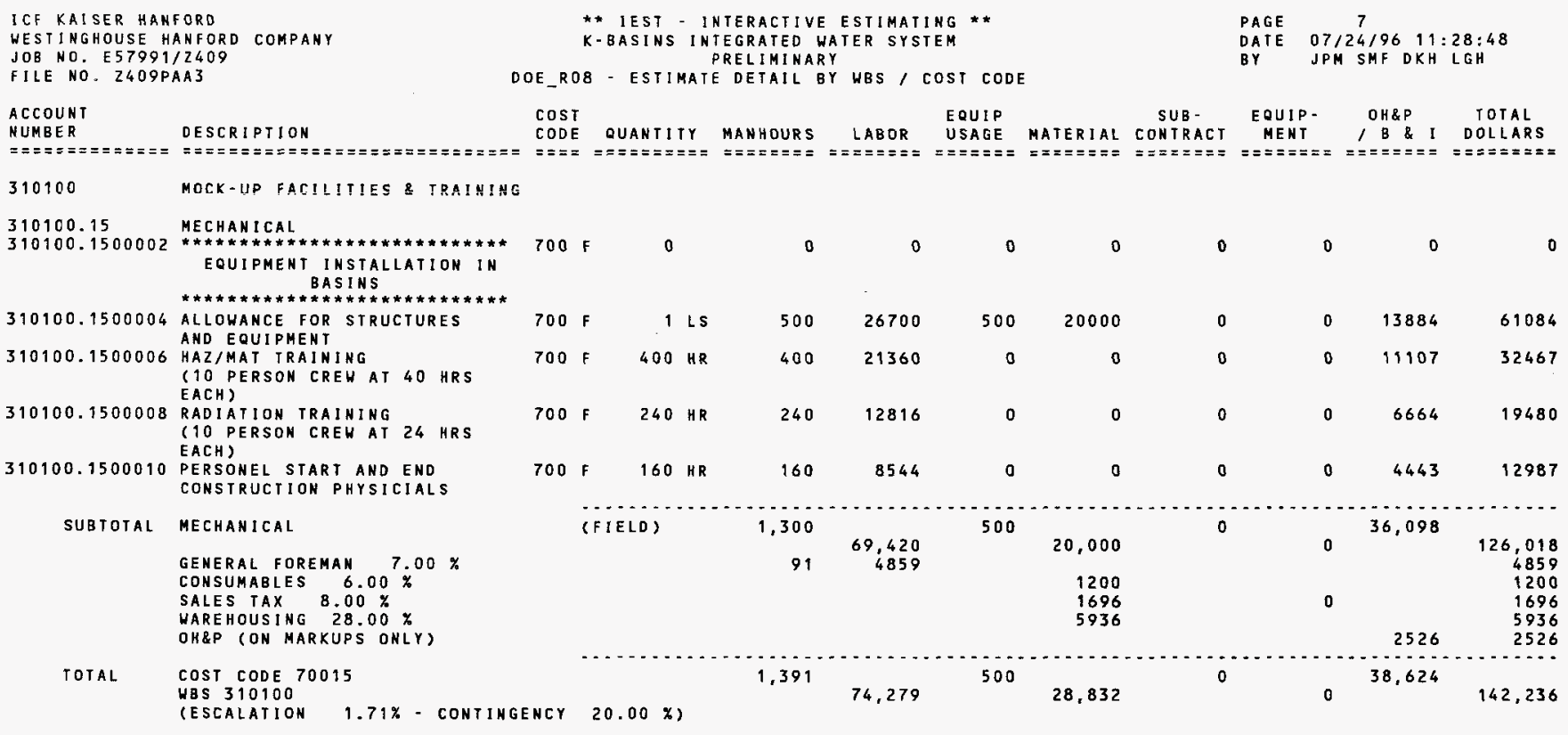

TOTAL HBS 310100 MOCK-UP FACILITIES \& TRAINING

$\begin{array}{llll}1,391 & 500 & & \\ & 74,279 & & 2832\end{array}$

o

38,624

142,236 
WHC-SD-SNF-RPT-012, Rev. 0

I CF KAISER HANFORD

WESTINGHOUSE HANFORD COMPANY

JOB NO. E57991/2409

FILE NO. Z409PAA3

ACCOUNT

NUMBER

DESCRIPTION

$==\mathbf{=}=\mathbf{=}==$

CHITLER BAY MODIFICATIONS

310101

$\begin{array}{ll}310101.13 & \text { SPECIAL CONSTRUCTION } \\ 310109.1333302 \text { PRE-ENGINEERED MODULE BLDG }\end{array}$ ENCLOSURE FOR FILTER CHANGE OUT

SUBTOTAL

\section{SPECIAL CONSTRUCTION}

SWP $20.00 \%$

GENERAL FOREMAN $7.00 \%$

CONSUMABLES $6.00 \%$

SALES TAX $8.00 \%$

WAREHOUSING $28.00 \%$

OH\&P (ON MARKUPS ONLY)

IOTAL COST CODE 50113

WBS 310101

CESCALATION

$1.71 \%-$ CONTINGENCY

501
* * IEST - INTERACTIVE ESTIMATING * *

K-BASINS INTEGRATED HATER SYSTEM

$$
\text { PREL I MINARY }
$$

DOE_ROB - ESTIMATE DETAIL BY WBS / COST CODE

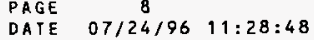

BY JPM SMF DKH LGH

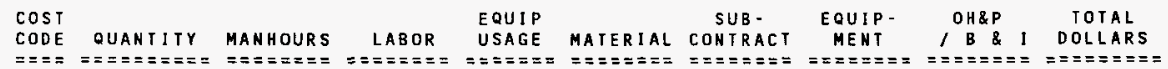

$\uparrow \mathrm{J} 0 \mathrm{~B}$

$7768 \quad 500 \quad 42000$

0

$0 \quad 4039$

54307

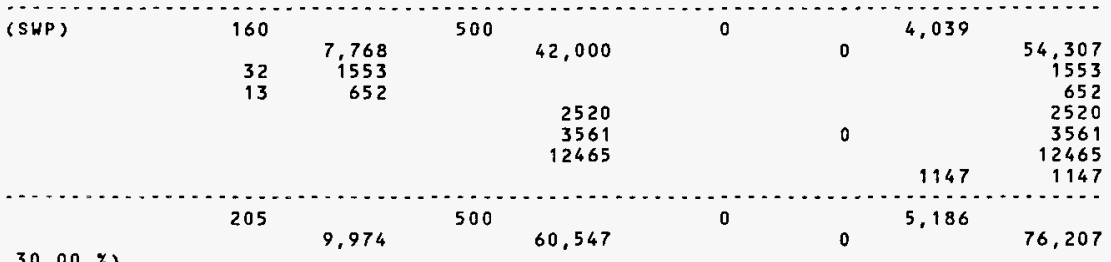

\subsection{5} MECHANICAL

310101.1581004 ECHANICAL

REMOVE \& SALVAGE PUMPS $* * * * * * * * * * * * * * * * * * * * * * * * * * * *$

310101.1581005 VALVE OFF PIPING \& DRAIN

310101.1581006 REMOVE 8"BOLT NUTS \& GSKT AT SUPPLY-RETURN OF EXIST PUMPS INCL B/FLANGE REMOVAL ON ROUGHED IN PUMP.

310101.1581008 REMOVE PUMPS

310101.1581010 ALLOWANCE FOR PUMP DECON

SUBTOTAL MECHANICAL

SWP $100.00 \%$

GENERAL FOREMAN $7.00 \%$

CONSUMABLES $6.00 \%$

SALES TAX $8.00 \%$

HARE HOUSING $28.00 \%$

OH\&P (ON MARKUPS ONLY)

TOTAL

COST CODE 70015

HBS 310101

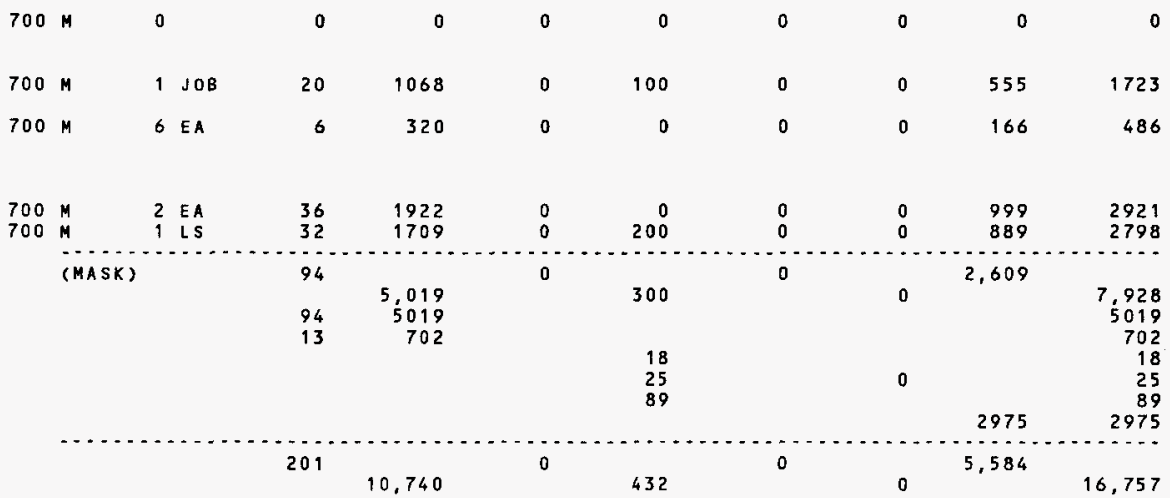

$c-24$ 
WHC-SD-SNF-RPT-012, Rev. 0

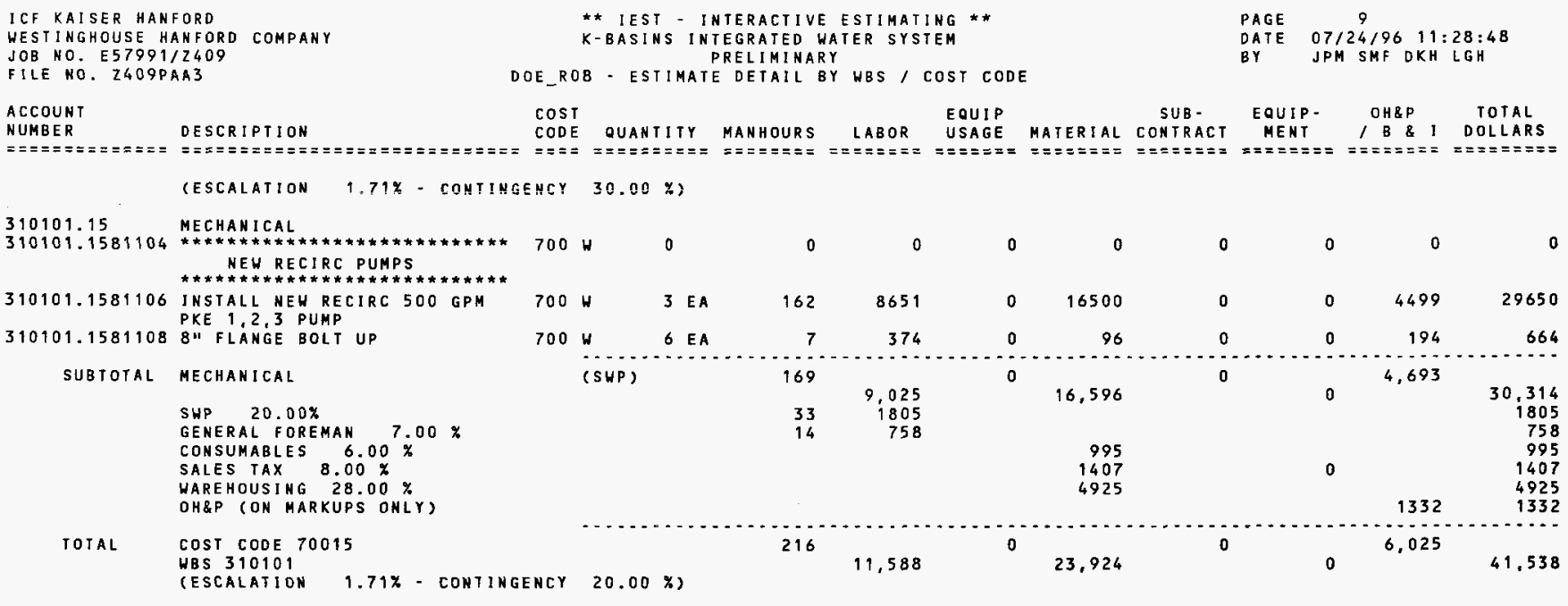

TOTAL HBS 310901 CHILLER BAY MODIFICATIONS

623

32,302

500

84,904

0

16,796

134,503 
WHC-SD-SNF-RPT-012, Rev. 0

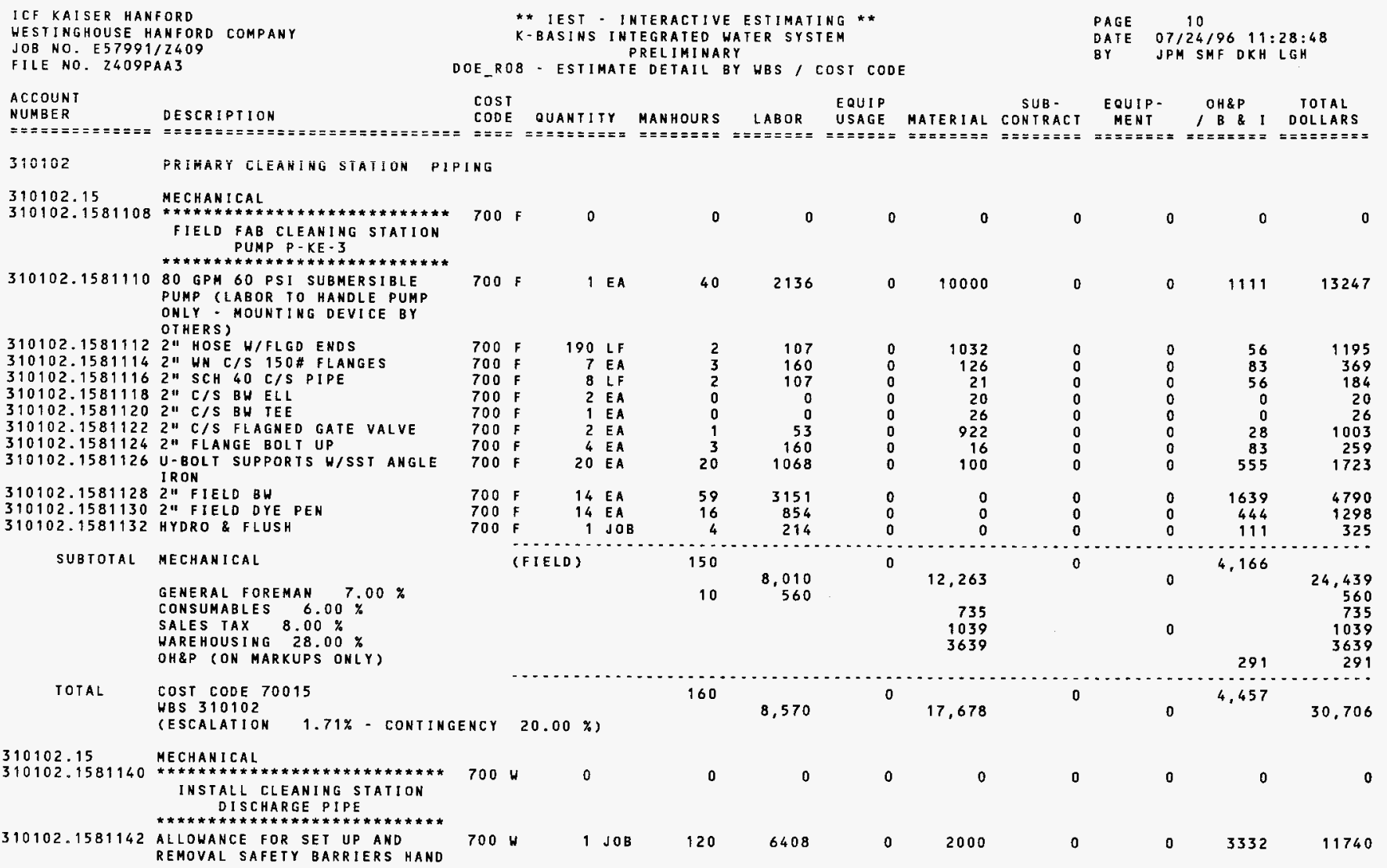


WHC-SD-SNF-RPT-012, Rev. 0

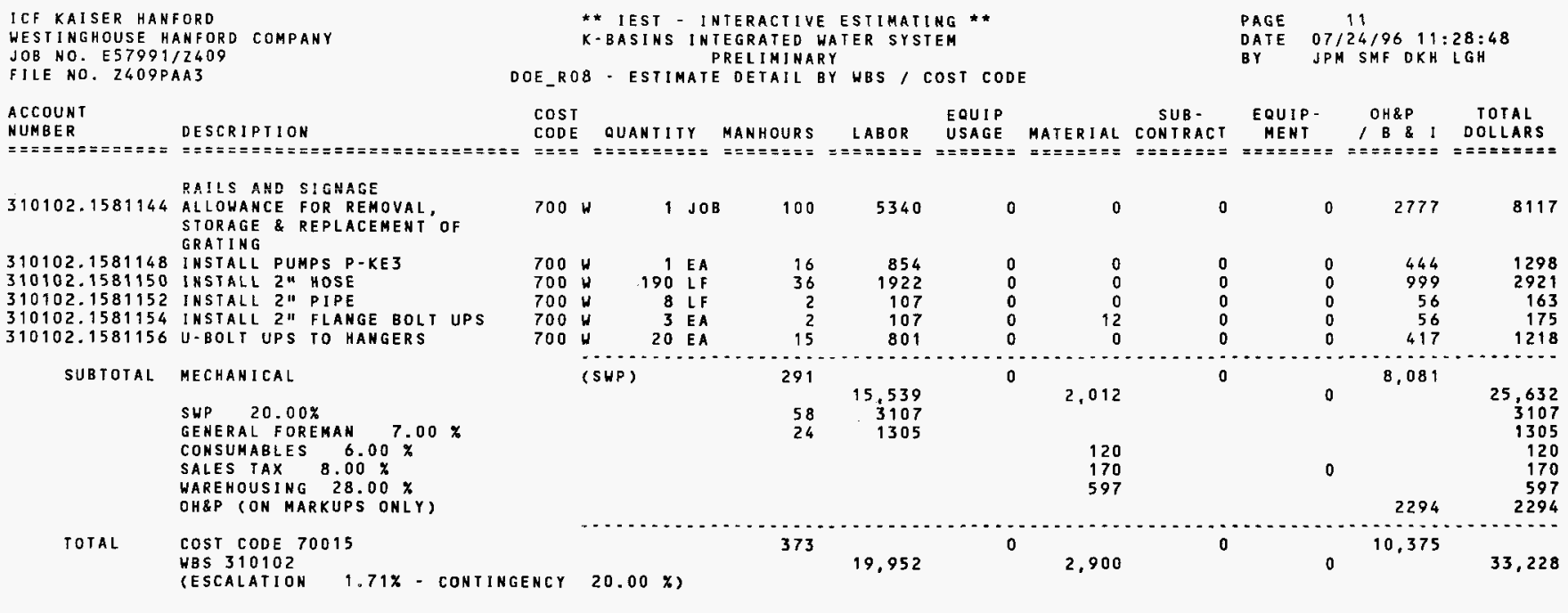

TOTAL WBS 310102 PRIMARY CLEANING STATION PIPING

534

28,522

0

20,578

0

14,833

63,934 
WHC-SD-SNF-RPT-012, Rev. 0

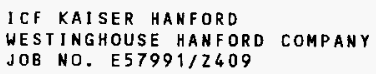

310103.1581144 HYDRO \& FLUSH

\section{SUBTOTAL MECHAHICAL}

GENERAL FOREMAN $7.00 \%$

CONSUMABLES $6.00 \%$

SALES TAX $8.00 \%$

WAREHOUSING $28.00 \%$

OH\&P (ON MARKUPS ONLY)

TOTAL

70015

HBS 310103

* IEST - INTERACTIVE ESTIMATING * *

K-BASINS INTEGRATED HATER SYSTEM

$\begin{array}{lcl}\text { PAGE } & 12 \\ \text { DATE } & 07 / 24 / 96 & 11: 28: 48\end{array}$ PRELIMIMARY

DOE_RO8 - ESTIMATE DETAIL BY HBS / COST CODE

BY JPM SMF DKH LGH

COST EQUIP SUB - EQUIP- OH\&P TOTAL

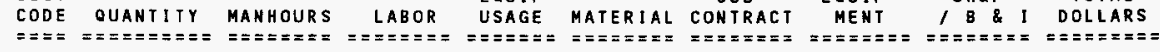

\begin{tabular}{|c|c|c|c|}
\hline $\begin{array}{l}700 \\
700\end{array}$ & $\begin{array}{l}F \\
F\end{array}$ & $\begin{array}{r}190 \\
1\end{array}$ & $\begin{array}{l}\text { LF } \\
\text { EA }\end{array}$ \\
\hline $\begin{array}{l}700 \\
700 \\
700 \\
700\end{array}$ & $\begin{array}{l}F \\
F \\
F \\
F\end{array}$ & $\begin{array}{r}24 \\
4 \\
2 \\
20\end{array}$ & $\begin{array}{l}\text { LF } \\
\text { LF } \\
\text { EA } \\
\text { EA }\end{array}$ \\
\hline $\begin{array}{l}700 \\
700 \\
700\end{array}$ & $\begin{array}{l}F \\
F \\
F\end{array}$ & $\begin{array}{l}1 \\
1 \\
1\end{array}$ & $\begin{array}{l}\text { EA } \\
E A \\
E A\end{array}$ \\
\hline $\begin{array}{l}700 \\
700 \\
700 \\
700 \\
700 \\
700 \\
700 \\
700\end{array}$ & $\begin{array}{l}F \\
F \\
F \\
F \\
F \\
F \\
F \\
F\end{array}$ & $\begin{array}{r}1 \\
1 \\
4 \\
4 \\
2 \\
12 \\
12 \\
1\end{array}$ & $\begin{array}{l}\text { EA } \\
\text { EA } \\
\text { EA } \\
\text { EA } \\
\text { EA } \\
\text { EA } \\
\text { EA } \\
\text { J DB }\end{array}$ \\
\hline
\end{tabular}

(FIELD)
0

18
84
3
80

9780

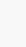

$\begin{array}{rr}2 & 107 \\ 1 & 53\end{array}$

827

$\begin{array}{rr}2 & 107 \\ 50 & 2670\end{array}$

153

$\begin{array}{rr}0 & 0 \\ 5 & 267\end{array}$
$1 \quad 427$

0

23247

107

960

160
107

107
4272

1175

320

227

$\begin{array}{rr}227 & 12,120 \\ 15 & 848\end{array}$

15

32,270

3
108
160

160

16
0

0

o

$0 \quad 0$

0

0

$\begin{array}{llll}0 & 0 & 56 & 1195\end{array}$

99

$0-222$

1936

2736

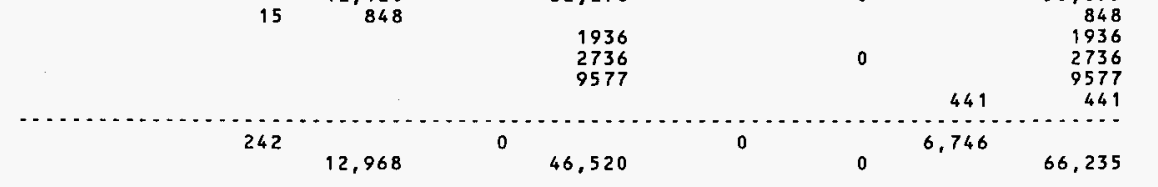


WHC-SD-SNF-RPT-012, Rev. 0

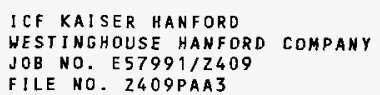

\section{ACCOUNT}

NUMBER

DESCRIPIION

CESCALATION

$1.71 \%$ - CONTINGENCY

* * IEST - INTERACTIVE ESTIMATING * *

K-BASINS JNTEGRATED WATER SYSIEM

PRELIMINARY

DOE_ROB - ESTIMATE DETAIL BY HBS, COSI CODE $\begin{array}{lcl}\text { PAGE } & 13 \\ \text { DATE } & 07 / 24 / 96 & 11: 28: 49\end{array}$

BY JPM SMF DKH LGH

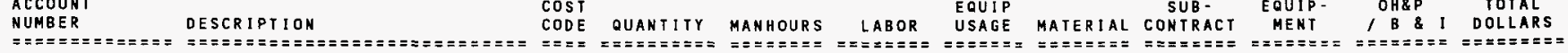

\begin{abstract}
310103.15 MECHANICAL
\end{abstract}

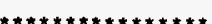

INSTALL DOHN DRAFT TABLE

PUMP DISCHARGE PIPE

310103.1581142 I**

310103.1581144411 SCH 40 C/S PIPE

310103.1581146 1-1/2" SCH 40 C/SPIPE

$310103.15811482 " 2$ HOSE

$310103.15811504 "$ FLANGE BOLT UP

$310103.1581152,1-1 / 2 "$ "FLANGE BOLT UP

310103.9581154 HYDRO \& FLUSH

310103.1581156 INSTALL PREFA8ED SUPPORTS

CHAIN, FLOATS SST ANGLE

310103.1581158 BOLT UP U BDLTS TO ANGLE IRN

310103.1581160 SAFETY BARRIERS REMOVAL AND REPLACEMENT OF GRATIMG IN

WBS 310102

SUBTOTAL MECHANICAL

SWP $20.00 \%$

GENERAL FOREMAN $7.00 \%$

CONSUMABLES $6.00 \%$

SALES TAX $8.00 x$

WAREHOUSING $28.00 \%$

OH\&P (ON MARKUPS OHLY)

TOTAL

COST CODE 70015

WBS 310103

700

0

0

o

o

0

0

0

0

\begin{tabular}{|c|c|c|c|}
\hline $\begin{array}{l}700 \\
700 \\
700 \\
700 \\
700 \\
700 \\
700 \\
700\end{array}$ & $\begin{array}{l}\mathbf{W} \\
\mathbf{H} \\
\mathbf{W} \\
\mathbf{W} \\
\mathbf{W} \\
\mathbf{W} \\
\mathbf{W} \\
\mathbf{W}\end{array}$ & $\begin{array}{r}1 \\
24 \\
4 \\
190 \\
4 \\
1 \\
1 \\
20\end{array}$ & $\begin{array}{l}\text { EA } \\
\text { LF } \\
\text { LF } \\
\text { LF } \\
\text { EA } \\
\text { EA } \\
\text { JOB } \\
\text { EA }\end{array}$ \\
\hline $\begin{array}{l}700 \\
700\end{array}$ & $\underset{W}{W}$ & $\begin{array}{r}20 \\
0\end{array}$ & EA \\
\hline
\end{tabular}

$\begin{array}{rrrr}20 & 1068 & 0 & 0 \\ 8 & 427 & 0 & \\ 1 & 53 & 0 & \\ 23 & 1228 & 0 & \\ 5 & 267 & 0 & 32 \\ 1 & 53 & 0 & 8 \\ 8 & 427 & 0 & 8 \\ 30 & 1602 & 0 & 0 \\ 20 & 1068 & 0 & \\ 0 & 0 & 0 & \end{array}$

0
0
0
0
0
0
0
0
0
0

$\begin{array}{rr}0 & 555 \\ 0 & 222 \\ 0 & 28 \\ 0 & 639 \\ 0 & 139 \\ 0 & 28 \\ 0 & 222 \\ 0 & 833 \\ 0 & 555 \\ 0 & 0\end{array}$

1623

649

1867

1867
438

89

657

2435

1623
0

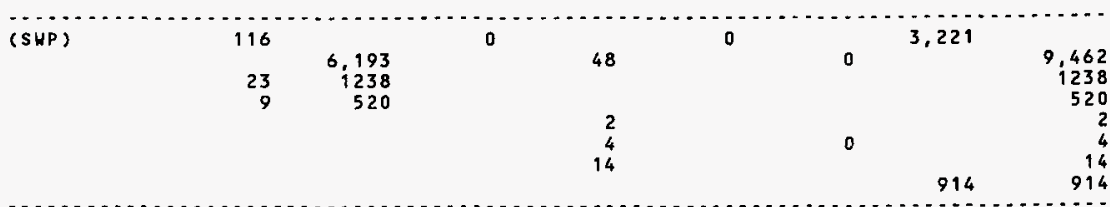

148

7,951

0

0

4,135

12,156

$20.00 \% 3$

$1.71 \%-$ CONTINGENCY

-

391

20,920

0

46,589

0

10,881

78,391 
WHC-SD-SNF-RPT-012, Rev. 0

ICF KAISER HANFORD
HESTINGHOUSE HANFORO COMPANY
JOB NO. E $57991 / 2409$

FILE NO. Z409PAA3
** IEST - INTERACTIVE ESTIMATING * *

K-BASINS INTEGRATED WATER SYSTEM

$$
\text { PRELIMINARY }
$$

DOE_ROB - ESTIMATE DETAIL BY WBS / COST CODE
PAGE $\quad 14,07 / 24 / 96 \quad 11: 28: 49$

BY JPM SMF OKH LGH

\begin{tabular}{|c|c|c|c|c|c|c|c|c|c|c|}
\hline $\begin{array}{l}\text { ACCOUNT } \\
\text { NUMBER } \\
==2===== \pm===\end{array}$ & $\begin{array}{l}\text { DESCRIPIION } \\
===\text { = = = = = = = = = = = = = = = = = = = = = }\end{array}$ & $\begin{array}{l}\operatorname{COST} \\
\operatorname{CODE} \\
====\end{array}$ & $\begin{array}{l}\text { QUANTITY } \\
=========\end{array}$ & $\begin{array}{l}\text { MANHOURS } \\
====\equiv==\end{array}$ & $\begin{aligned} & \text { LABOR } \\
&=\Xi====\end{aligned}$ & $\begin{array}{l}\text { EQUIP } \\
\text { USAGE } \\
======\end{array}$ & $\begin{array}{l}\text { MATERIAL } \\
=======\end{array}$ & $\begin{array}{c}\text { SUB - } \\
\text { CONTRACT } \\
=======\end{array}$ & $\begin{array}{c}\text { EQUIP - } \\
\text { MENT } \\
=======\end{array}$ & 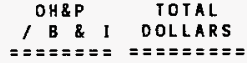 \\
\hline
\end{tabular}

310104

MISC DRAIN VEMT \& RETURN TO BASIN

310104.15 MECHAN I CAL

$310104.1581108 * * * * * * * * * * * * * * * * * * * * * * * * * * * * 700$ FIELD FAB HISC ORAIN AND VENT RETURN PIPING

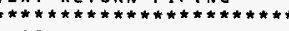

310104.1581110 q" SCH 40 C/S PIPE 310104.1581112 " "THREADED C/S ELL. 310104.158111411 "THREADED C/S TEE 310104.1581116 HYDRO \& FLUSH

310104.1581118 7" RELIEF VALVES

$310104.15811202 "$ SCH 40 C/S PIPE

310104.15811222 " IHREADED C/S ELL

$310104.15811242 " \mathrm{C} / S$ FIELD BH

310104.958112621 DYE PEN

310104.1581128 HYDRO \& FLUSH

310104.1581130 FAB 2" PIPE SUPPORTS

310104.1581132 FAB 11 PIPE SUPPORTS

SUBTOTAL MECHANICAL

GENERAL FOREMAN $7.00 \%$

CONSUMABLES $6.00 \%$

SALES TAX $8.00 x$

WAREHOUSING $28.00 \%$

OH\&P (ON MARKUPS ONLY)

TOTAL COST CODE 70015

HBS 310104

(ESCALATION $1.71 \%$ - CONTINGENCY $30.00 \%$ )

$\begin{array}{rrrrr}700 & F & 60 & \text { LF } \\ 700 & F & 10 & \text { EA } \\ 700 & F & 2 & \text { EA } \\ 700 & F & 1 & \text { JOB } \\ 700 & F & 3 & \text { EA } \\ 700 & F & 30 & \text { LF } \\ 700 & F & 6 & \text { EA } \\ 700 & F & 10 & \text { EA } \\ 700 & F & 10 & \text { EA } \\ 700 & F & 2 & \text { EA } \\ 700 & F & 6 & \text { EA } \\ 700 & F & & 4 & \text { EA }\end{array}$

0

0

0

0

0

(FIELD)

4 EA

$\begin{array}{rrr}8 & 427 & 0 \\ 1 & 53 & 0 \\ 1 & 53 & 0 \\ 3 & 160 & 0 \\ 3 & 160 & 0 \\ 6 & 320 & 0 \\ 1 & 53 & 0 \\ 42 & 2243 & 0 \\ 11 & 587 & 0 \\ 6 & 320 & 0 \\ 12 & 641 & 0 \\ 8 & 427 & 0\end{array}$

33
15
4
0
450
79
30
0
0
0
30
15

$\begin{array}{ll}0 & 0 \\ 0 & 0 \\ 0 & 0 \\ 0 & 0 \\ 0 & 0 \\ 0 & 0 \\ 0 & 0 \\ 0 & 0 \\ 0 & 0 \\ 0 & 0 \\ 0 & 0 \\ 0 & 0\end{array}$

222
28

28

83

83
166

28

1166

305

166

333
222

15

0

2,830

5,444
381

656

0

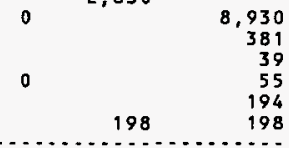

109

39
55
194

198

3,028 198 MECHANICAL

310104.15 INSTALL MISC DRAIN \& VENT

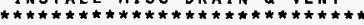

310104.1581306 1" SCH 40 C/S PIPE

310104.1581308 HYDRO \& FLUSH

310104.1581310 INSTALL 1 "PIPE SUPPORTS

310104.1581312 2" PIPE SPOOLS

310104.1581314 2" FIELD HELDS

310104.158131621 "OYE PEN

$\begin{array}{rrrr}700 W & 0 & \\ 700 \mathrm{H} & 60 & \text { LF } \\ 700 \mathrm{~W} & 1 & \text { EA } \\ 700 \mathrm{H} & 6 & \text { EA } \\ 700 \mathrm{H} & 30 & \text { LF } \\ 700 \mathrm{H} & 2 & \text { EA } \\ 700 \mathrm{H} & 2 & \text { EA }\end{array}$

5,825

945

0

9,798

$\begin{array}{ccc}0 & 0 & 0 \\ 8 & 427 & 0 \\ 3 & 160 & 0 \\ 5 & 267 & 0 \\ 6 & 320 & 0 \\ 8 & 427 & 0 \\ 2 & 107 & 0\end{array}$


WHC-SD-SNF-RPT-012, Rev. 0

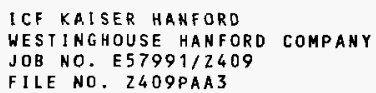

20

2,082

416
174

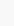

1,082

3. 184

416

$\begin{array}{ll}7 & 416 \\ 3 & 174\end{array}$

1
1
5

307

50

2,673

0

28

0

, 389

0

$20.00 x)$
158
8,498
0

974

0

4,417

13,890 
WHC-SD-SNF-RPT-012, Rev. 0

I CF KAISER HANFORD

WESTINGHOUSE HANFORD COMPANY JOB NO. E57991/2409

FILE NO. Z 409 PAA3
** IEST - INTERACIIVE ESTIMAIING * *

K-BASINS INTEGRATED HATER SYSTEM

$$
\text { PRELIMINARY }
$$

DOE_ROB - ESTIMATE DETAIL BY WBS / COST CODE

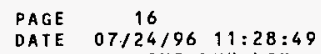

BY JPM SMF DKH LGH

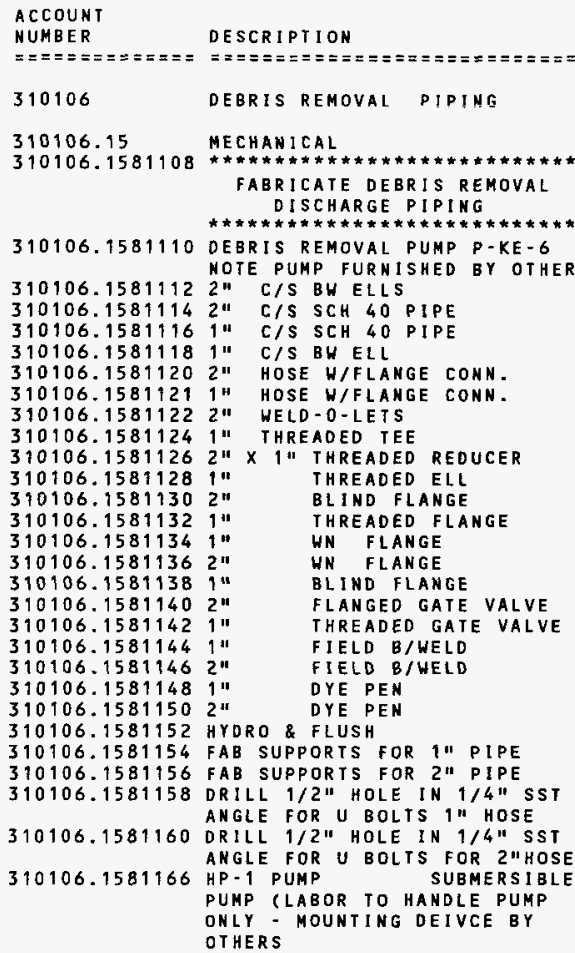

310106.1581167 PUMP FURNISHED BY OTHERS

40

\section{6}

$\begin{array}{llll}700 \mathrm{~F} & 12 \mathrm{EA} & 2 & 107\end{array}$

$700 \mathrm{~F} \quad 80 \mathrm{LF} \quad 15 \quad 809$

$700 \mathrm{~F} 20 \mathrm{LF} \quad 3 \quad 160$

$700 \mathrm{~F} \quad 0 \quad 0 \quad 0$

$\begin{array}{llll}700 \mathrm{~F} & 60 \mathrm{LF} & 6 & 320\end{array}$

$700 \mathrm{~F} \quad 2$ EA $\quad 1 \quad 53$

$700 \mathrm{~F} T \mathrm{EA}$

$700 \mathrm{~F}$ EA

$700 \mathrm{~F}$

700

700

$700 \mathrm{~F}$

$700 \mathrm{~F}$

$700 \mathrm{~F}$

$700 \mathrm{~F}$

$700 \mathrm{~F}$

700

700

700

$700 \mathrm{~F}$

$700 \mathrm{~F}$

$700 \mathrm{~F}$

$700 \mathrm{~F}$

$700 \mathrm{~F}$

1 EA

$700 \mathrm{~F}$

0

185


WHC-SD-SNF-RPT-012, Rev. 0

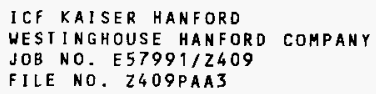

* * IEST - INTERACTIVE ESTIMATING * *

K-BASINS INTEGRATED HATER SYSTEM

DATE $07 / 24 / 96 \quad 11: 28: 49$

PRELI IMINARY

DOE_ROB - ESTIMATE DETAIL BY HBS, COST CODE

BY JPM SMF DKH LGH

\section{ACCOUNT \\ NUMBER \\ DESCRIPTION}

$\cos T$

QUANTITY MANHOURS LABOR USAGE MAIERIAL COHT- EQUTP- OH\&P TOTAL

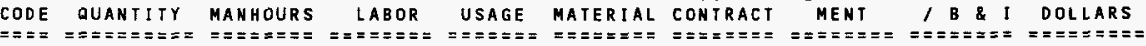

$== \pm=$
SUÉTUT́AL MECHANICAL

GENERAL FOREMAN $7.00 \%$

CONSUMABLES $6.00 \%$

SALES TAX $8.00 \%$

HAREHOUSING $28.00 \%$

OH\&P (ON MARKUPS ONLY)

TOIAL

COST CODE 70015
HBS 310106

CESCALATION $1.71 \%$ - CONTINGENCY
(FIELD) 393

27

27

20,983
MECHAN I CAL

310106.1581300

VALVE OFF EXISTING PIPING

AND DRAIN LINES FOR TIE INS

310106.1581302 ALLOHANCE FOR GLOVE BAG

310106.1581303 2" SADDEL WELD

$310106.95813042 "$ OYE PEN

310106.1581306 2" WELD-0-LETS

310106.1581308 REMOVE \& PKG GLOVE BAG FOR

BURIAL (INCL BURIAL DRUH)

SUBTOTAL MECHAHICAL

GENERAL FOREMAN $7.00 *$

CONSUMABLES $6.00 \%$

SALES TAX $8.00 \%$

WAREHOUSING $28.00 \%$

OH\&P (ON MARKUPS ONLY)

TOTAL

COST CODE 70015

WBS 310106

$1.71 \%$ - CONTINGENCY $30.00 \%$

$700 \mathrm{M}$ (MASK)

\section{TIE INS}

SWP $100.00 \%$

$\begin{array}{rrrrrrr}700 \mathrm{M} & 0 & 0 & 0 & 0 & 0 \\ 700 \mathrm{M} & 1 \text { JOB } & 24 & 1282 & 0 & 50 \\ 700 \mathrm{M} & 1 \text { LS } & 8 & 427 & 0 & 250 \\ 700 & \text { M } & 2 \text { EA } & 10 & 534 & 0 & 4 \\ 700 \mathrm{M} & 2 \text { EA } & 2 & 107 & 0 & 4 \\ 700 \mathrm{M} & 2 \text { EA } & 1 & 53 & 0 & 4 \\ 700 \mathrm{M} & 1 \text { J OB } & 4 & 214 & 0 & 110\end{array}$

49

2,617
2617

0

422

25

25
35

125

104

5. 600

0

608

0

0

0

667

1999

0

0
0
0

0
0

0
0

0

222
278

278

58
28
111

111

816

816
167

167
85

85
435

\subsection{5} MECHANICAL

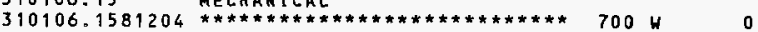

INSTALL DEBRIS PUNP
0

0

o

0

0

0


WHC-SD-SNF-RPT-012, Rev. 0

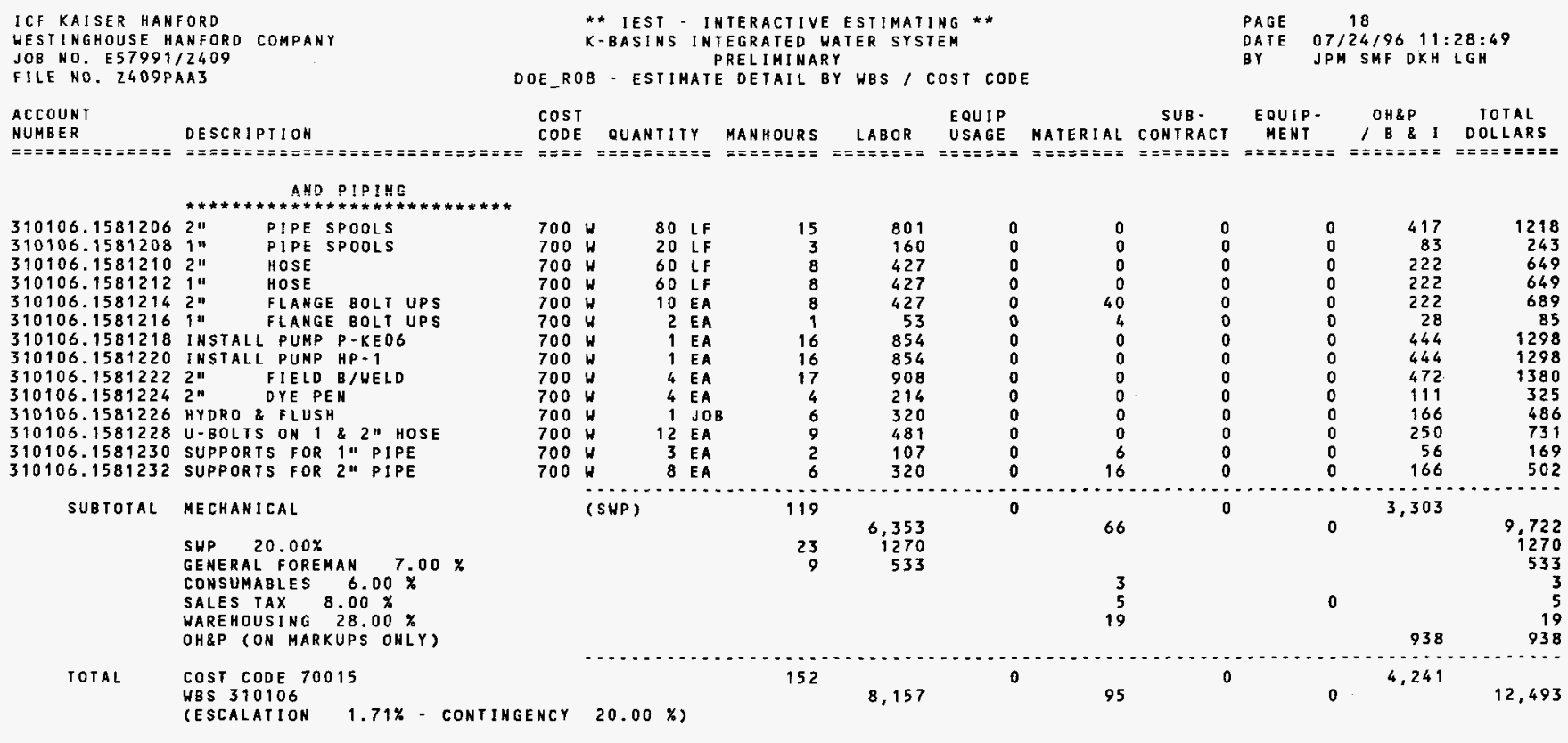


WHC-SD-SNF-RPT-012, Rev. 0

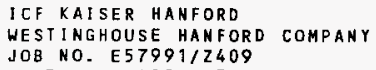

FILE NO

ACCOUNT

NUMBE

DESCRIPTION

WEASEL PIT PUMP \& PIPING

310107 MECHANICAL

310107.15

340107.1581108

FABRICATE WEASEL PIT PUMP DISCHARGE PIPING

310107.1581190 WEASEL PIT PUMP P-KE (LABOR TO HANDLE PUMP ONLY MOUNTING DEVICE BY OTHERS

310107.15811123 " HOSE W/CONNECT IONS

310107.1581114 4" SCH 40 C/S PIPE

310107.158111631 SCH 40 C/S PIPE

310107.158111820 SCH 40 C/S PIPE

310107.15811202 " C/S BW ELLS

310107.15811223 " C/S BW ELLS

310107.1581124 4"X 3 " BH C/S RED. TEE

$310107.15811264^{\prime \prime}$

310107.158112821

$310107.95811303 \%$

$310107.15811322 "$

$310107.15811344 "$

$310107.75811362 "$

310107.158113831

$310107.15811404^{\prime \prime}$

310107.158114221

310107.158114431

$310107.15811464 "$

$310107.15811483 "$

$310107.15811502 "$

$310107.15811524^{\prime \prime}$

BH C/S WELD-D-LET

HN C/S FLANGES

WH C/S FLANGES

C/S BLIND FLANGE

WN C/S FLANGE

FLANGED GATE VALVE

FLANGED GATE VALVE

FLANGED GATE VALVE

FIELD B / WELD

FIELD B/WELD

FIELD B/WELD

DYE PEN

DYE PEN

DYE PEN

310107.1581154 DRILL HOLES IN SST ANGLE FOR U-BOLT AND FAB ANGLE IRON

310107.1581156 FAB PIPE SUPPORTS FOR 2" CIS PIPE

310107.1581158 FAB PIPE SUPPORTS FOR 31 CIS PIPE

310107.1581160 2" FLANGE BOLT UP 310107.1581162 3" FLANGE BOLT UP 310107.1581164 4" FLANGE BOLT UP 310107.1581166 HYORO \& FLUSH

SUBTOTAL MECHANICAL
* * IEST - INTERACTIVE ESTIMATING * *

K-BASINS INTEGRATED WATER SYSTEM PRELIMINARY

DOE_RO - ESTIMATE DETAIL BY WBS/ COST CODE
PAGE 19

DATE $07 / 24 / 96 \quad 11: 28: 49$

$8 Y$

CODE QUANTITY MANHOURS LABOR USAGE

SUB -

SUB -
CONTRACT

EQUIP- OH\&P TOTAL $=============0======$

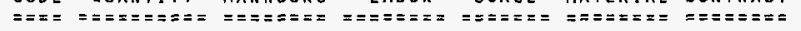

700

1 EA

2136

23247

700

$\begin{array}{lll}700 & F & 40 \mathrm{LF}\end{array}$

$700 \mathrm{~F} \quad 10 \mathrm{LF}$

$700 \mathrm{~F} \quad 10 \mathrm{LF}$

$2 \mathrm{EA}$

$700 \mathrm{~F} \quad 4$ EA

$700 \mathrm{~F} \quad 1 \mathrm{EA}$

$700 \mathrm{~F} \quad 6$ EA

$700 \mathrm{~F} 3 \mathrm{EA}$

$700 \mathrm{~F} \quad 1 \mathrm{EA}$

$700 \mathrm{~F} \quad 3 \mathrm{EA}$

$700 \mathrm{~F} I \mathrm{EA}$

1 EA

$700 \mathrm{~F} \quad 1 \mathrm{EA}$

$700 \mathrm{~F} \quad 9 \mathrm{EA}$

$700 \mathrm{~F} \quad 12 \mathrm{EA}$

$700 \mathrm{~F} \quad 12 \mathrm{EA}$

$700 \mathrm{~F} \quad 9 \mathrm{EA}$

$700 \mathrm{~F}$

$\begin{array}{ll}700 & \mathbf{F} \\ 700 & \mathrm{~F}\end{array}$

4 EA

$\begin{array}{ll}4 & E A \\ 5 & E A\end{array}$

153

4214

160
107

107
0

53
0

0

160

107

0

107
53

53
53

53

2029

3578

1388

908

534

374

$700 \mathrm{~F}$

$2 E A$

107

$700 F \quad 2$ EA

107

$700 \mathrm{~F} 3$ EA

$700 \mathrm{~F}$

$\begin{array}{ll}700 & F \\ 700 & F\end{array}$

$700 \mathrm{~F}$

(FIELD)

3 EA

$2 E A$

3 EA

252

13,456

107

53
107

641

$\begin{array}{rr}0 & 421 \\ 0 & 35\end{array}$

30
26

20

64
47
25

180

108

22

120

461

655
849

0

0

0

0

0
75

0
0

0
0
0

0
0

0
0
0

0
0
0

o

0
0

0
0
0

0
0

o

0
0
0

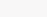

(-2.

$<-3 i$ 
WHC-SD-SNF-RPT-012, Rev. 0

ICF KAISER HANFORD

HESTINGHOUSE HANFORD COMPANY

JOB NO. E57991/Z409

FILE NO. Z409PAA3

ACCOUNT

NUMBER DESCRIPTION
* * IEST - INTERACTIVE ESTIMATING * *

K-BASINS INTEGRATED HATER SYSTEM

$$
\text { PRELIMINARY }
$$

DOE ROB - ESTIMATE DETAIL BY HBS / COST CODE
PAGE 20

DATE $07 / 24 / 96 \quad 11: 28: 50$

BY JPM SMF DKH LGH

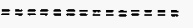

GENERAL FOREMAN $7,00 \%$

CONSUMABLES $6.00 \%$

SALES TAX $8.00 \%$

UAREHOUSING $28.00 \%$

OH\&P (ON MARKUPS ONLY)

TOTAL

COST CODE 70015
HBS 310107

CESCALATION

$1.71 x-$ CONTINGENCY

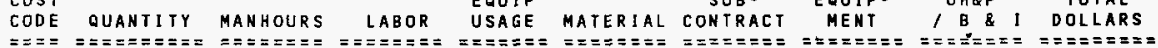

SUB -

EQUIP- OH\&P TOTAL

310107.15 MECHANICAL

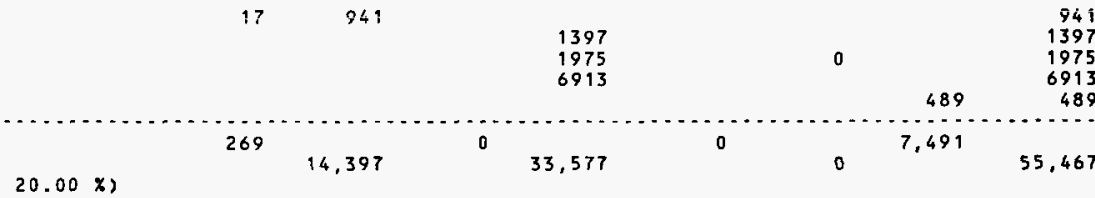

\section{TIE INS}

$700 M$

310107.1581235 VALVE OFF EXISIING PIPING
AHD DRAIN LINES FOR TIE INS

310107.1581235 VALVE OFF EXISIING PIPING
AHD DRAIN LINES FOR TIE INS

310107.1581236 PLACE GLOVE BAG

310107.15812374 " C/S WELD-D-LET

310107.15812384 4" SADDLE HELD

310107.1581240 ALLOHANCE FOR REMOVAL OF G/BAG AND PKG FOR BURIAL

SUBTOTAL

\section{MECHANICAL}

SWP $100.00 \%$

GENERAL FOREMAN $7.00 \%$

CONSUMABLES $6.00 \%$

SALES TAX $8.00 \%$

WAREHOUSING $28.00 \%$

OH\&P (ON MARKUPS ONLY)

TOTAL

COST CODE 70015

HBS 310107

(ESCALATION

1.71\% - CONTINGENCY

700
700
700
700

0

0

0

0

0

0

0

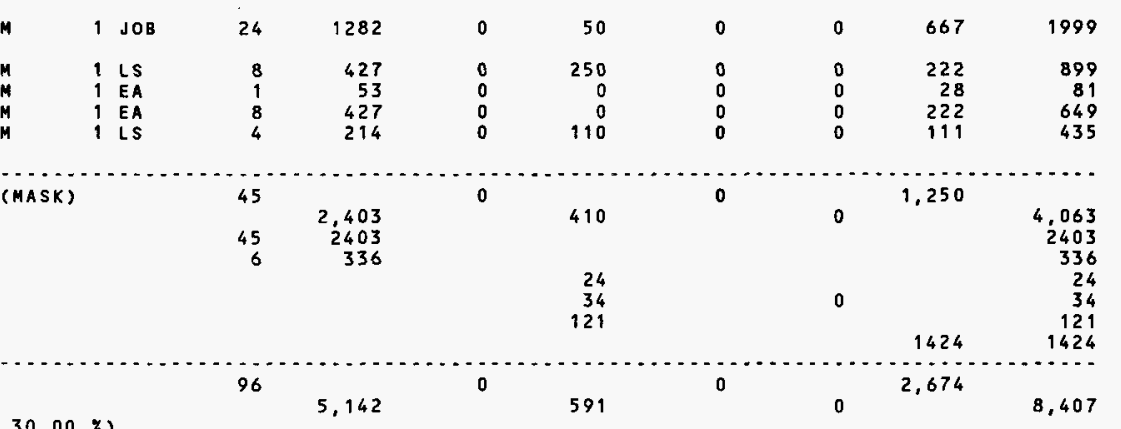

310107.15

MECHANICAL

310107.1581204

\section{INSTALL WEASEL PUMP}

AND PIPING

310107.1581206 HANDLE 3 "HOSE

310107.1581208 HANDLE 3 " PIPE SPOOLS

700

0

0

0

0

0

0

0

$700 \mathrm{~W} \quad 40 \mathrm{LF}$

$\begin{array}{ll}6 & 320 \\ 3 & 160\end{array}$

0
0

0
0

0

0
0

0
0

$\begin{array}{rr}0 & 166 \\ 0 & 83\end{array}$

486
243 
WHC-SD-SNF-RPT-012, Rev, 0

I CF KAISER HANFORD

WESTINGHOUSE HANFORD COMPANY

JOB NO. E5799१/2409

FILE NO. Z409PAA3

\section{ACCOUNT}

NUMBER

DESCRIPTION

310107.1581210 HANOLE 2" PIPE SPOOLS

310107.1581212 INSTALL PUMP PK- $2 E$

310107.1581214 2" FIELD C/S B/HELD

310107.158121631 FIELD C/S B/WELD

310107.1581218211 DYE PEN

310107.15812203 " OYE PEN

310107.1581222411 DYE PEN

310107.158122424 2" FLANGE BOLT UP

310107.15812263 "

310107.1581228 INSTALL 3" HOSE U-BOLTS

310107.1581230 INSTALL 3"PIPE SUPPORT

310107.9581232 INSTALL 2" PIPE SUPPORT

310107.1581233 HYDRO \& FLUSH

\section{SUBTOTAL MECHANICAL}

\section{SHP $20.00 \%$}

GENERAL FOREMAN $7.00 \%$

CONSUMABLES $6.00 \%$

SALES TAX $8.00 \%$

WAREHOUSING $28.00 \%$

OH\&P (ON MARKUPS ONLY)

TOTAL

\section{COST CODE 70015}

HBS 310107

(ESCALAI ION

$1.71 \%$ - CONTINGENCY

$20.00 \%)$
* * IEST - INTERACTIVE ESTIMATING * *

K-BASINS INTEGRATED WATER SYSTEM

PRELIMINARY

DOE RO8 - ESTIMATE DETAIL BY WBS / COST CODE
PAGE 21

DATE $07 / 24 / 96 \quad 11: 28: 50$

BY JPM SMF DKH LGH

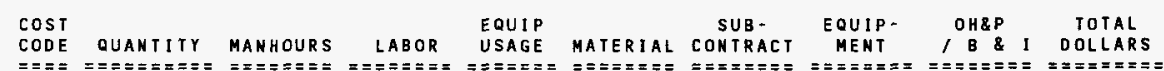

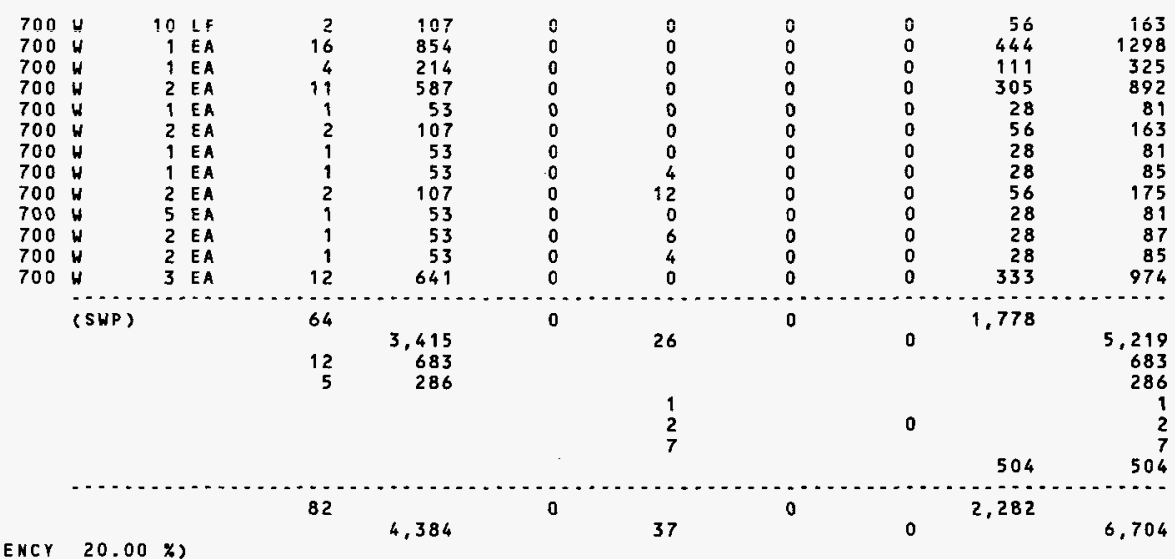

TOTAL UBS 310107 WEASEL PIT PUMP \& PIPING

448

23,925

0

34,206

12,448

70,580 
WHC-SD-SNF-RPT-012, Rev. 0

ICF KAISER HANFORD
HESTINGHOUSE HANFORD COMPANY
JOB NO. EST $91 / 2409$
FILE NO. Z4O9PAA3

ACCOUNT

NUMBER

DESCRIPTION

HYOROCYCLONES \& PIPING

310108

310108.15

MECHANICAL

HYDROCYCLONES \& ASSOCIATED PI P ING

$\star * \star * * * * * * *$

310108.1581052 HYDROCYCLONES SKE-1A, 1B, $1 \mathrm{C}$

310108.1581054 HYDROCYCLONES SKE-

$310108.15810568 " \quad$ U-BOLTS

310108.1581058 FAB \& DRILL C/S PLATE FOR $\mathrm{U}=\mathrm{BOL}$ IS
S

310108.1581060 4" SCH 40 C/S PIPE

$310108.15810623 " 1$ SCH 40 C/S PIPE

$310108.15810642 "$ SCH 40 C/S PIPE

$310108.15810664 \mathrm{H}$ C/S BW ELL

$310108.15810683 "$ C/S BW ELL

$310108.15810702 " \quad C / S$ BW ELL

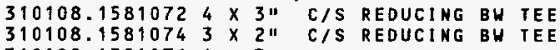

$310108.15810764 \times 3 "$ C/S RECUCER

$310108.15810783 \times 2$ " C/S RECUCER

$310108.15810804 " \quad$ C/S HN FLANGE

310108.951084211

310108.158108640 " FIELD B/WELD

$310108.15810883^{\prime \prime}$ FIELD B/HELD

$310108.15810902 "$ FIELD B/WELD

$310108.15810924^{\prime \prime}$

310108.1581094311

$310108.15810962 "$

310108.15810983 "

$310108.15811002 "$

$310108.15811024^{\prime \prime}$

310108.158110431

310108.158110621

310108.1581108

310108.1581108 HYDRO \& FLUSH
310108.1581110 FAB 4" SUPPOR

310108.1581112 FAB 3 " SUPPORT

310108.1581114 FAB 2" SUPPORTS

SUBTOTAL MECHANICAL. DYE PEN DYE PEN

FLANGED GATE VALVE FLANGED GATE VALVE FLANGE BOLT UPS FLANGE BOLT UPS LANGE
BOLT UPS
LANGE BOLT UPS

-15
$S U P P O R T S$
* * IEST - INTERACTIVE ESTIMATING * *

K-BASINS INTEGRATED HATER SYSTEM PRELIMINARY

DOE_ROB - ESTIMATE DETAIL BY WBS/ COST CODE
PAGE 22

DATE $07 / 24 / 96 \quad 11: 28: 50$

BY JPM SMF DKH LGH

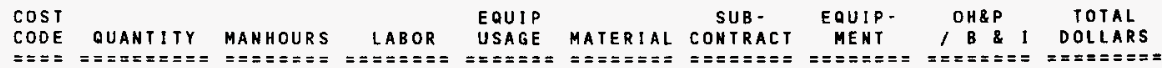

$700 F \quad 0$

0

0

o

0

\section{0}

$700 \mathrm{~F} \quad 1$ EA

$700 \mathrm{~F} \quad 4 \mathrm{EA}$

4 EA

$\begin{array}{ll}6 & 320 \\ 2 & 107 \\ 2 & 107 \\ 5 & 267\end{array}$

$700 \mathrm{~F} \quad 30 \mathrm{LF}$

$700 \mathrm{~F} \quad 60 \mathrm{LF}$

$700 \mathrm{~F} \quad 40 \mathrm{LF}$

$700 \mathrm{~F} \quad 3 \mathrm{EA}$

$700 \mathrm{~F} \quad 3$ EA

$700 \mathrm{~F} \quad 10 \mathrm{EA}$

$700 \mathrm{~F} 3 \mathrm{EA}$

$700 \mathrm{~F} 2 \mathrm{EA}$

$700 \mathrm{~F} I \mathrm{EA}$

$700 \mathrm{~F}$

$700 \mathrm{~F} \quad 1 \mathrm{EA}$

$700 \mathrm{~F} \quad 7$ EA

$700 \mathrm{~F}$

$700 \mathrm{~F}$

$700 \mathrm{~F}$

$700 \mathrm{~F}$

$700 \mathrm{~F}$

$700 \mathrm{~F}$

$700 \mathrm{~F}$

700

700

$700 \mathrm{~F}$
$700 \mathrm{~F}$

$700 \mathrm{~F}$
$700 \mathrm{~F}$

$700 \mathrm{~F}$

700

$700 \mathrm{~F}$

7 EA

13 EA

21 EA

27 EA

13 EA

21 EA

$1 E A$

1 EA

1 EA

1 EA

$1 \mathrm{EA}$

6 EA

3 EA

6 EA

$11 \quad 587$

$\begin{array}{rr}17 & 908 \\ 8 & 427\end{array}$

153

$\begin{array}{rr}1 & 53 \\ 2 & 107\end{array}$

107
53

53

1
0

4 EA

(FIELO)

EA

$30 \quad 1602$

153

153

$\begin{array}{rr}1 & 53 \\ 24 & 1282\end{array}$

$\begin{array}{rr}24 & 1282 \\ 3 & 160\end{array}$

$6 \quad 320$

$498 \quad 26,589$

0

37800
14000
120
120

0
0
0
0

0
0
0

38286

$\begin{array}{rr}56 & 14163 \\ 56 & 283 \\ 139 & 526\end{array}$

105

180

105

48

100

180

94
20
14

14
40

245

107

592

6034

634
228

210

0
0
0

0
0
0

0

655

460

8
6
4

6
4
0

20

120

0

55,045

0

....

13.832

$\begin{array}{rr}305 & 99 \\ 472 & 156 \\ 222 & 75\end{array}$

997
1560

754
162

162
129

129
263

263

261
175

20

14 
WHC-SD-SNF-RPT-012, Rev. 0

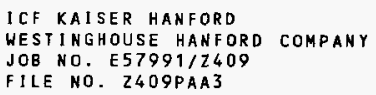

ACCOUNT

$\begin{array}{ll}\text { NUMBER } & \text { DESCRIPTION } \\ ===== \pm===== & =========\end{array}$

* IEST - INTERACTIVE ESTIMATING * *

K-BASINS INTEGRATED WATER SYSTEM

PRELIMINARY

DOE_ROB - ESTIMATE DETAIL BY WBS / COST CODE

CODE QUANTITY MANHOURS LABOR USAGE

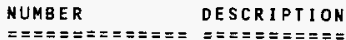

$\begin{array}{lcl}\text { PAGE } & 23 \\ \text { DAIE } & 07 / 24 / 96 & 11: 28: 50\end{array}$

BY JPM SMF DKH LGH

MATERIAL CONTRAC

SUB -

EQUIP- OH\&P TOTAL MENT, $B$ \& DOLLARS

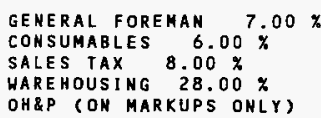

TOTAL

\section{COST CODE 70015 \\ WBS 310108} SESCALATION

310108.15

$\begin{array}{ll}310108.15 & \text { MECHANICAL } \\ 310108.1581104 * * * * * * * * * * * * * * * * * * * * * * * * * * * & 700 H\end{array}$

INSTALL HYDROCYCLONES

$$
\text { AND PIPING }
$$

310108,1581106 INSTALL HYDROCYCLONES

310908.1581108 INSIALL 4 " PIPE SPOOLS

310108.1581110 INSTALL 3 "PIPE SPOOLS

310108.1581112 INSTALL 2" PIPE SPOOLS

$310108.15811144^{\prime \prime}$

FIELD B/WELO

310108.1581116 3" FIELD B/HELD

310108.1581118 2" FIELO B/WELD

$310108.15811204 "$ OYE PEN

310108.158112231 DYE PEN

310108.158112420 DYE PEH

310108.1581126 HYDRO \& FLUSH

310108.158112831 FLANGE BOLT UPS

310108.15811302 " FLANGE BOLT UPS
FLANGE BOLT UPS

310108.1581132 IHSTALL 4" PIPE SUPPORTS

310108.1581134 INSTALL 3 " PIPE SUPPORTS

310108.9581136 INSTALL 2" PIPE SUPPORTS

\section{SUBTOTAL MECHANICAL}

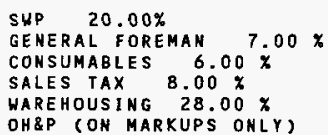

TOTAL

$\operatorname{COST} \operatorname{CODE} 70015$

WBS 310108

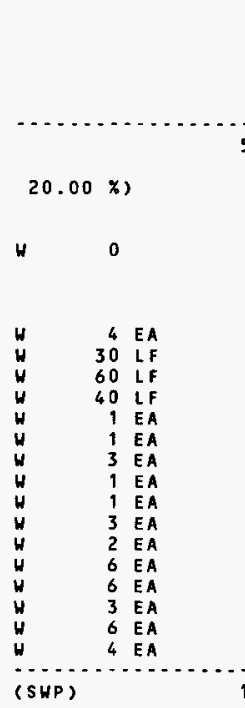

$34 \quad 9861$

3302
$461 \quad$

4667

532

28,450

0

79,352

= $=$ = = = = =

o

$\begin{array}{ll}24 & 1282 \\ 11 & 587\end{array}$

$\begin{array}{ll}11 & 587 \\ 17 & 908\end{array}$

$8 \quad 427$

7
6

$13 \quad 694$

$2 \quad 107$

$3 \quad 160$

2641

$\begin{array}{ll}5 & 267 \\ 5 & 267\end{array}$

$\begin{array}{ll}3 & 160 \\ 6 & 320\end{array}$

$4 \quad 214$

$\begin{array}{rr}0 & 0 \\ 0 & 0 \\ 0 & 0 \\ 0 & 0 \\ 0 & 0 \\ 0 & 0 \\ 0 & 0 \\ 0 & 0 \\ 0 & 0 \\ 0 & 0 \\ 0 & 0 \\ 0 & 36 \\ 0 & 24 \\ 0 & 9 \\ 0 & 12 \\ 0 & 8\end{array}$

127

0

0

0

89

0

.525

$\begin{array}{rr}667 & 1949 \\ 305 & 892 \\ 472 & 1380 \\ 222 & 649 \\ 194 & 568 \\ 166 & 486 \\ 361 & 1055 \\ 56 & 163 \\ 28 & 81 \\ 83 & 243 \\ 333 & 974 \\ 139 & 442 \\ 139 & 430 \\ 83 & 252 \\ 166 & 498 \\ 111 & 333\end{array}$

$\begin{array}{rr}25 & 6,789 \\ 10 & 1356 \\ & 569\end{array}$

89

0

10, 395

1356
569

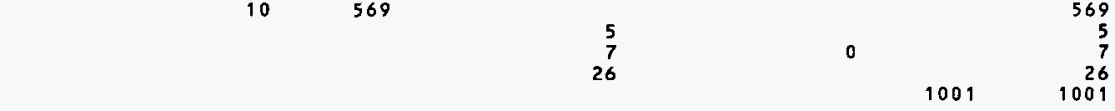

163

8,706

0

128

0

4,526

13,361 
WHC-SD-SNF-RPT-012, Rev. 0

ICF KAISER HANFORD

WESTINGHOUSE HANFORD COMPANY

JOB NO. E $57991 / 2409$

FILE NO. 2409 PAA3

ACCOUNT

NUMBER DESCRIPTIOK

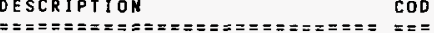

(ESCALATION

$1.71 X-$ COMTINGENCY

* * IEST - INTERACTIVE ESTIMATING * *

K-BASINS INTEGRATED WATER SYSTEM PRELIMINARY DOE_ROB - ESTIMATE DETAIL BY WBS / COST CODE

COST EQUIP

CODE QUANTITY MANHOURS

LABOR

USAGE

SUB-
MATERIAL CONTRACT

$20.00 \%)$
PAGE 24

DATE $07 / 24 / 96 \quad 11: 28: 50$

BY JPM SMF DKH LGH
TOTAL HBS 310108 HYOROCYCLONES \& PIPING

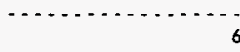

69
37,157
0

(1)

79,481
19,326

135,964 
WHC-SD-SNF-RPT-012, Rev. 0

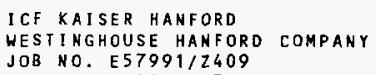

* * IEST - INTERACTIVE ESTIMATING * *

K-BASINS INTEGRATEO WATER SYSTEM PRELIMINARY

DOE ROB - ESTIMATE DETAIL BY HBS / COST CODE $\begin{array}{lc}\text { PAGE } & 25 \\ \text { DATE } & 07 / 24 / 96 \quad 11: 28: 50\end{array}$

BY JPM SMF OKH LGH

\begin{tabular}{|c|c|c|c|c|c|c|c|c|c|}
\hline $\begin{array}{l}\text { ACCOUNT } \\
\text { NUMBER } \\
============2\end{array}$ & 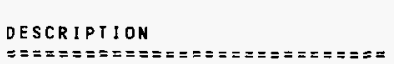 & $\begin{array}{l}\cos T \\
\operatorname{coD} \\
====\end{array}$ & $\begin{array}{l}\text { QUANTITY } \\
========\end{array}$ & $\begin{array}{l}\text { MANHOURS } \\
==\approx====\equiv\end{array}$ & $\begin{aligned} & \operatorname{LABOR} \\
===\equiv=== & ==\end{aligned}$ & $\begin{array}{l}\text { EQUIP } \\
\text { USAGE } \\
==\Xi=\approx==\end{array}$ & $\begin{array}{l}\text { MATERIAL } \\
==\approx==\approx==\end{array}$ & $\begin{array}{l}\text { SUB: } \\
\text { CONTRACT } \\
=====\approx==\end{array}$ & 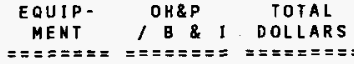 \\
\hline
\end{tabular}

310109

FILTER BACKWASH PIPING

310109.15

MECHANICAL

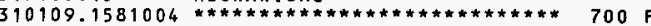

FIELO FABRICATE

\section{BACKUASH SYSTEH}

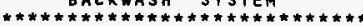

310109.158900624

SCH $40 \mathrm{C} / \mathrm{S}$ PIPE

310109.1581008311

SCH $40 \mathrm{C} / \mathrm{S}$ PIPE

310109.158101031

C/S BW ELL

310109.1581012311

C/S BW TEE

310109.158101631

310109.158101820

C/S WH FLANGE

C/S WN FLANGE

C/S BW ELL

FLANGED GAIE VALVE

$310109.15810202 "$

310109.7581022 3"

$310109.15810243 "$

LANGED GATE VALVE

$80 L T$ UPS

BOLT UPS

C/S BH TEE

310109.1581028

AL FILTER BACK FLUSH

IANK, ASME GLASS LINED 125

PSE 42" DIA $\times 96$ " LONG

310109.1581031 ALLOH FOR $5 \times 8 \times$ I CONC.

RAD BARRIER AT FILTER

310109.1581032 SAFETY RELIEF VALVE

$310109.1581034211 \times 3 "$ BH C/S CONC REDUCER 310109.158103620 GPM P-KE-7 BACKWASH PUMP

310109.1581038 HYDRO \& FLUSH

310109.1581040 FAB SUPPORTS

310109.1581042 3" FIELD B/HELD

$310109.15810442 "$ " FIELD B/WELD

310109.158104631 DYE PEN

310109.1581048 2" DYE PEN

SUBTOTAL MECHANICAL

GENERAL FOREMAN $7.00 \quad x$

CONSUMABLES $6.00 \%$

SALES TAX $8.00 \%$

HARE HOUSING $28.00 \%$

OH\&P (ON MARKUPS ONLY)

TOTAL

COSI CODE 70015

WBS 310109
$700 \mathrm{~F} \quad 0$

$\begin{array}{rrrr}700 & F & 20 & \text { LF } \\ 700 & F & 20 & \text { LF } \\ 700 & F & 6 & \text { EA } \\ 700 & F & 4 & \text { EA } \\ 700 & F & 20 & \text { EA } \\ 700 & F & 9 & \text { EA } \\ 700 & F & 6 & \text { EA } \\ 700 & F & 2 & \text { EA } \\ 700 & F & 5 & \text { EA } \\ 700 & F & 10 & \text { EA } \\ 700 & F & 4 & \text { EA } \\ 700 & F & 1 & \text { EA } \\ 700 & F & 1 & \text { EA }\end{array}$

0

0

o

$\begin{array}{rr}4 & 214 \\ 6 & 320 \\ 2 & 107 \\ 1 & 53 \\ 10 & 534 \\ 4 & 214 \\ 1 & 53 \\ 1 & 53 \\ 5 & 267 \\ 8 & 427 \\ 3 & 160 \\ 0 & 0 \\ 15 & 801\end{array}$

0
0
0
0
0
0
0
0
0
0
0
0
0

53
60
96
144
700
270
60
920
3280
60
16
26
3900

700

$6 \mathrm{CY}$

50

2670

$\begin{array}{ll}700 & F \\ 700 & F\end{array}$

$\begin{array}{ll}700 & F \\ 700 & F\end{array}$

$700 \mathrm{~F}$

$700 \mathrm{~F}$

$700 \mathrm{f}$

$700 \mathrm{~F}$

$700 \mathrm{~F}$

$700 \mathrm{~F}$

(FIELD)

1 EA

1 EA

1 EA

6 EA

44 EA

25 EA

25 EA

44 EA
25 EA

EA

$1 \quad 53$

$\begin{array}{rr}1 & 53 \\ 0 & 854 \\ 16 & 854\end{array}$

$16 \quad 854$

6
246
13136

$62 \quad 3311$

1495

28

$41 \quad 31,503$
2205

$0 \quad 420$

150

15
5000

0
180

180
0

0

0

0

15.350

0

$\begin{array}{rr}41 & 2205 \\ & 921 \\ & 1301 \\ 4555\end{array}$

631

33,708

0

22,128

o

17,529

o
$105 \quad 5607$ 
WHC-SD-SNF-RPT-012, Rev. 0

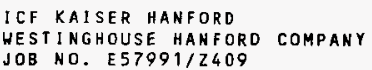

* IEST. INTERACTIVE ESTIMATING * *

K-BASINS INTEGRATED HATER SYSTEM

$$
\text { PRELIMINARY }
$$

DOE ROB - ESTIMATE DETAIL BY WBS / COST CODE

\section{$\begin{array}{lc}\text { PAGE } & 26 \\ \text { DATE } & 07 / 24 / 96 \quad 11: 28: 50\end{array}$}

BY JPM SMF DKH LGH

\begin{tabular}{|c|c|}
\hline $\begin{array}{l}\text { ACCOUNT } \\
\text { NUMBER } \\
===========0\end{array}$ & 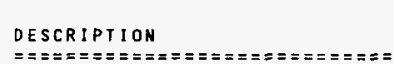 \\
\hline
\end{tabular}

(ESCALATION

\section{$\cos T$ \\ EOUIP}

CODE QUANTITY MANHOURS

$\angle A B O R$

USAGE

SUB-
MATERIAL CONTRACT

EQUIP- OH\&P TOTAL MENT $/$ B \& I DOLLARS $1.71 \%$ - CONTINGENCY $20.00 \%$ )

310109.15 MECHANICAL

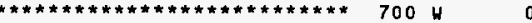

FIELO INSTALL BACK WASH SYSTEM

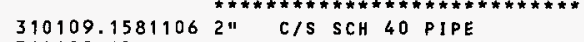
$310109.158110831 \mathrm{C} / \mathrm{S}$ SCH 40 PIPE 310109.15811102 310109.158119231 BOLT UPS

310109.1581114 INSIALL $2 "$ SUPPORIS

310109.1581116 HYDRO \& FLUSH

SUBTOTAL MECHANICAL

\section{SWP $20.00 X$}

GENERAL FOREMAN $7.00 \%$

CONSUAABLES $6.00 \%$

SALES TAX 8.00\%

WAREHOUSIHG $28.00 \%$

OH\&P (ON MARKUPS OHLY)

TOTAL

COST COOE 70015

HBS 310109

CESCALATION

$1.71 \%$ - CONIINGENCY $20.00 \%$

\begin{tabular}{|c|c|c|c|}
\hline $\begin{array}{l}700 \\
700 \\
700 \\
700 \\
700 \\
700\end{array}$ & $\left\{\begin{array}{l}W \\
W \\
W \\
W \\
W\end{array}\right.$ & $\begin{array}{r}20 \\
20 \\
5 \\
5 \\
6 \\
2\end{array}$ & $\begin{array}{l}\text { LF } \\
\text { LF } \\
E A \\
E A \\
E A \\
E A\end{array}$ \\
\hline
\end{tabular}

0

o

0

0

0

0

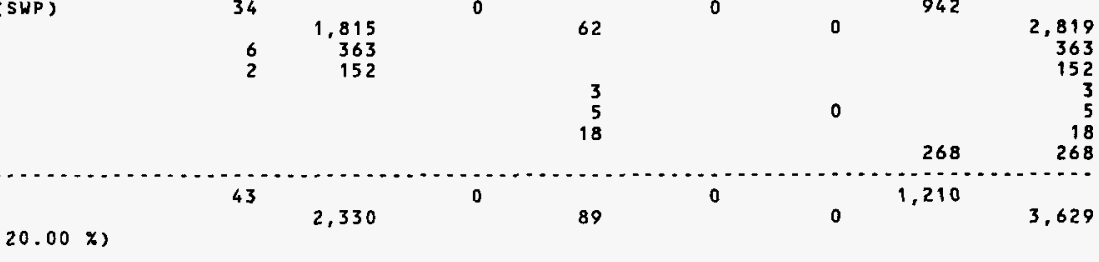

TOTAL WBS 310109 FILTER BACKWASH PIPING

674

36,038

0

22,217

0

76,996 
WHC-SD-SNF-RPT-012, Rev. 0

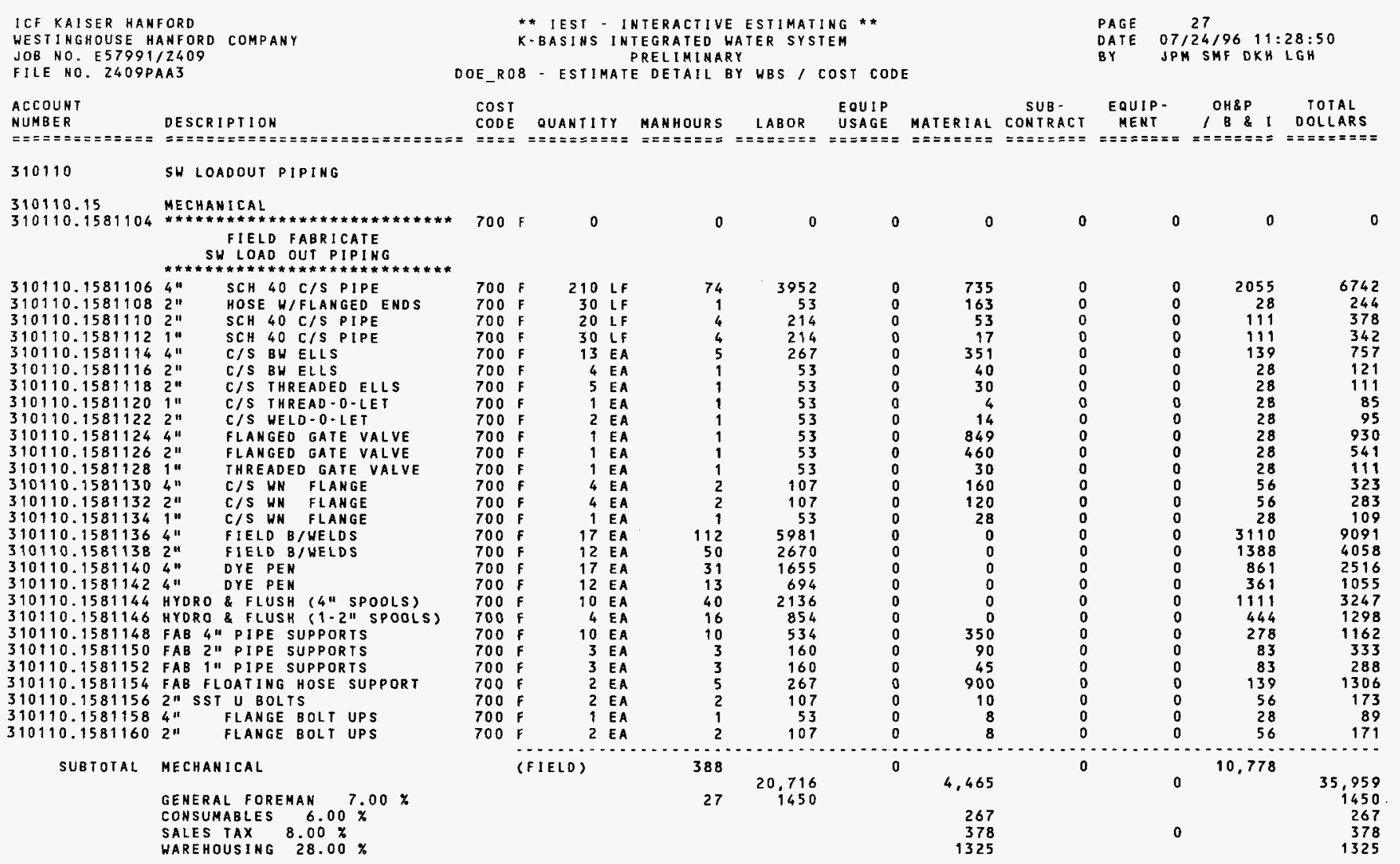


WHC-SD-SNF-RPT-012, Rev. 0

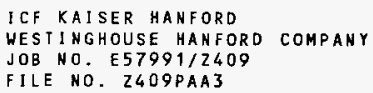

310110.1581038 ALLOUANCE FOR SET UP AND REMOVAL SAFETY BARRIERS HAND RAILS AND SIGNAGE

310110.1581039 ALLOWANCE FOR REMOVAL, STORAGE \& REPLACEMENT'OF GRATING

\section{SUBTOTAL MECHANICAL}

SHP $20.00 \%$

GENERAL FOREMAN $7.00 \%$

CONSUMABLES $6.00 \%$

SALES TAX $8.00 \%$
$700 \mathrm{~W} \quad 0$

$700 \mathrm{H} \quad 210 \mathrm{LF}$

$700 \mathrm{H} 20 \mathrm{LF}$

$700 \mathrm{~W} \quad 30 \mathrm{LF}$

$700 \mathrm{~W} \quad 30 \mathrm{LF}$

$700 \mathrm{~W} \quad 3 \mathrm{EA}$

$700 \mathrm{H}$
$700 \mathrm{H}$

$700 \mathrm{H} \quad 23$ EA

$700 \mathrm{H} \quad 4$ EA

$700 \mathrm{H}$ 23

$700 \mathrm{H} 270 \mathrm{LF}$

$700 \mathrm{H} 2$ EA

$700 \mathrm{~W}$

700

$700 \mathrm{H}$
$700 \mathrm{H}$

10 EA

$\begin{aligned} 10 & \text { EA } \\ 3 & \text { EA }\end{aligned}$

3 EA

3 EA

$1 \mathrm{JOB}$

$700 \mathrm{~W}$

$1 \mathrm{JOB}$

$700 \mathrm{~W}$

415

415

22,166

0

6.436

00

74
4
4
4
4
2
152
17
41
4
11

3952

214

214

214

107

8117

908

2189

214
587

$8 \quad 427$

$10 \quad 534$

$\begin{array}{ll}2 & 107 \\ 5 & 267\end{array}$

60

3204

2670

0

50

0

0

455

24,299

4859
2041

91
38
3160
2,080

124
176
0

\begin{abstract}
0
\end{abstract}
754

11,532

754

0,134

0

0
0
0
0
24
8
0
0
0
0
0

0
0
0
0
0
0
0
0
0
0
0

0

$\begin{array}{lll}0 & 111 & 349\end{array}$

0

o

0
0
0

4221

472

1138

191
305

12338

1380

3327

0

o

222

892

30

6
6
6

0

649

278

83
56

56
139

842

249

169
412

2000

1666

6870

o

o

1388

4058 $\begin{array}{rrr}0 & 2055 & 6007 \\ 0 & 111 & 325\end{array}$

111
111 o

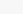

(n)

12,634

39.013

4859
2041

2041
124 176 
WHC-SD-SNF-RPT-012, Rev. 0

I CF KAISER HANFORO

WEST INGHOUSE HANFORD COMPANY

JOB NO. E57991/2409

FILE NO 7409 PAA3

\section{ACCOUNT \\ NUMBER}

DESCRIPI ION
* * IEST - InTERACTIVE ESTIMATING * *
K-BASINS INTEGRATED HATER SYSTEM PRELIMINARY

PAGE 29

DATE $07 / 24 / 96 \quad 11: 28: 51$

DOE_RO8 - ESTIMATE DETAIL BY WBS / COST CODE
BY

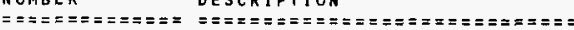

COST EQUIP

COOE QUANTITY MANHOURS LABOR USAGE

MATERIAL CONTRACT EQUIP- OH\&P TOTAL

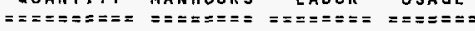

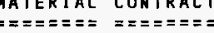

WAREHOUSING $28.00 \%$

OH\&P (ON MARKUPS ONLY)

TOTAL

COST CODE 70015

HBS 310110

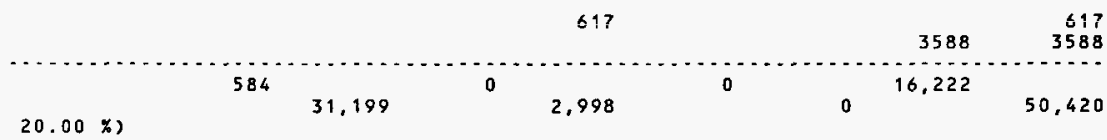

TOTAL WBS 310110 SW LOADOUT PIPIHG

999

53,366 o

9,435

0

27,754

90,555 
WHC-SD-SNF-RPT-012, Rev. 0
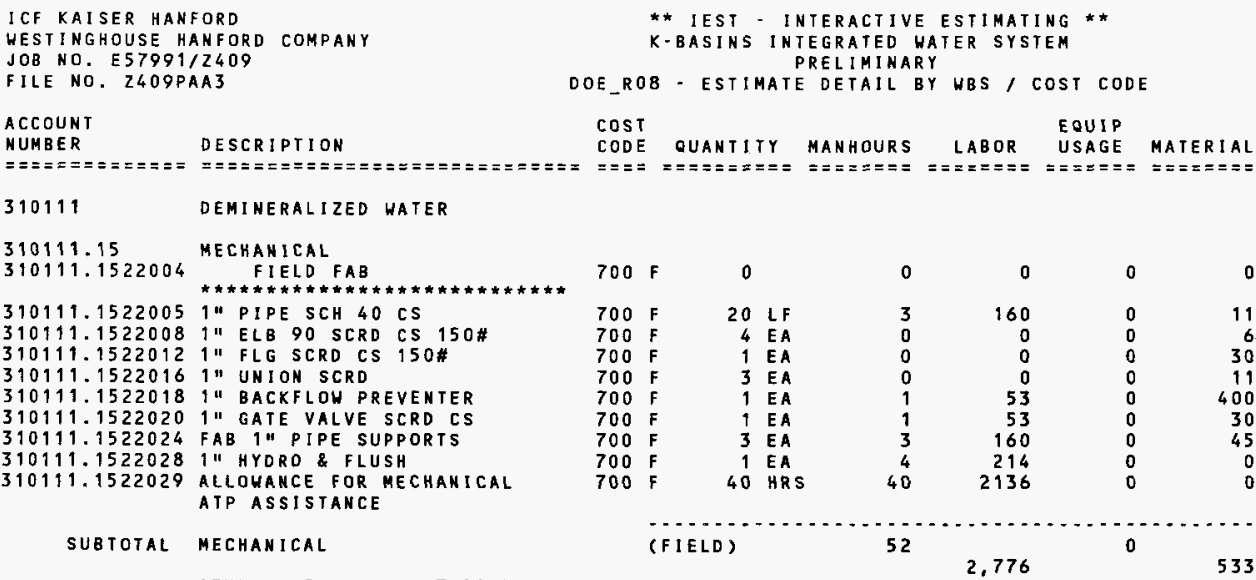

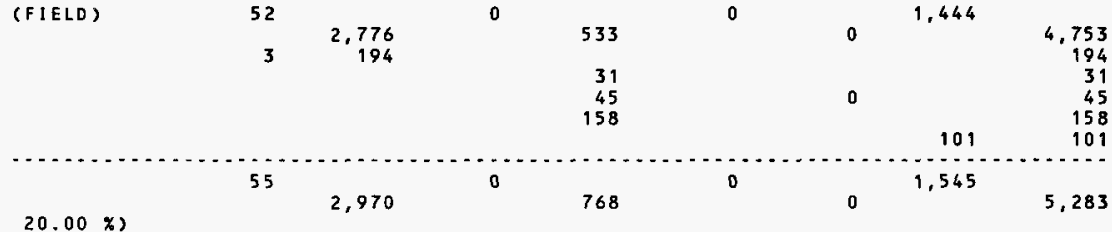

PAGE 30

DATE $07 / 24 / 96 \quad 11: 28: 51$

BY JPM SMF DKH LGH

\subsection{5 MECHANICAL}

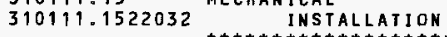

SUBTOTAL MECHANICAL

SUP $\quad 20.00 \%$

\begin{tabular}{|c|c|c|c|c|c|c|c|c|c|c|c|}
\hline 700 & $W$ & 0 & & 0 & 0 & 0 & 0 & 0 & 0 & 0 & 0 \\
\hline $\begin{array}{l}700 \\
700 \\
700 \\
700\end{array}$ & $\begin{array}{l}W \\
W \\
W \\
W\end{array}$ & $\begin{array}{r}20 \\
\mathbf{3} \\
1 \\
1\end{array}$ & $\begin{array}{l}\text { LF } \\
\text { EA } \\
E A \\
E A\end{array}$ & $\begin{array}{l}3 \\
2 \\
3 \\
4\end{array}$ & $\begin{array}{l}160 \\
107 \\
160 \\
214\end{array}$ & $\begin{array}{l}0 \\
0 \\
0 \\
0\end{array}$ & $\begin{array}{l}0 \\
9 \\
0 \\
0\end{array}$ & $\begin{array}{l}0 \\
0 \\
0 \\
0\end{array}$ & $\begin{array}{l}0 \\
0 \\
0 \\
0\end{array}$ & $\begin{array}{r}83 \\
56 \\
83 \\
111\end{array}$ & $\begin{array}{l}243 \\
172 \\
243 \\
325\end{array}$ \\
\hline \multirow[t]{3}{*}{700} & W & 1 & EA & 16 & 854 & 0 & 5000 & 0 & 0 & 444 & 6298 \\
\hline & (SHP) & & & 28 & & $\overline{0}$ & & $\overline{0}$ & 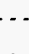 & 777 & \\
\hline & & & & 5 & $\begin{array}{r}495 \\
299\end{array}$ & & 5,009 & & 0 & & $\begin{array}{r}7.281 \\
299\end{array}$ \\
\hline
\end{tabular}


WHC-SD-SNF-RPT-012, Rev. 0

ICF KAISER HANEORD

WEST INGHOUSE HANFORD COMPANY

JOB NO. E57991/2409

FILE NO $2409 P A A 3$

* * IEST - INTERACTIVE ESTIMATING * *

K-BASINS INTEGRATEO HATER SYSTEM

$$
\text { PRELIMINARY }
$$

DOE_ROB - ESTIMATE DETAIL BY UBS, COST CODE

$\cos$

ACCOUNT

NUMBER

DESCRIPTION

QUANTITY MANHOURS

$14 B 08$

EQUIP

$==\approx===$

MATERIAL CONTRACT

DATE $07 / 24 / 96 \quad 19: 28: 51$

BY

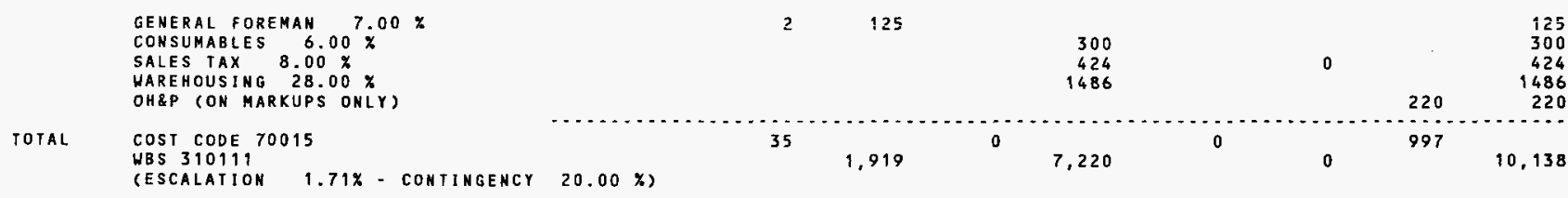

TOTAL WBS 310111 DEMINERALIZED WATER

91

4.889

o

7,989

2,542

15,422 
WHC-SD-SNF-RPT-012, Rev. 0

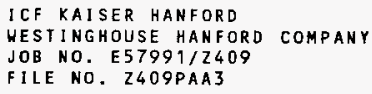

\section{ACCOUNT}

NUMBER

DESCRIPI ION

* * IEST - INTERACTIVE ESTIMATING * *

$K$-BASINS INTEGRATED HATER SYSTEM PREL IMINARY

DOE_ROB - ESIIMATE DEIAIL BY WBS / COST CODE

CODE SUB- EQUIP- OH\&P TOTAL

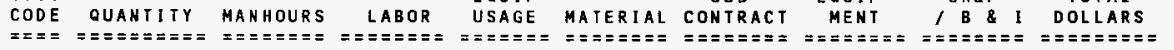

310112 WEASEL PIT MODIFICATIONS

310112.15 MECHANICAL

310112.1500054 HEASEL PIT BARRIER

310112.1500056 TRANSPORT BARRIER INSIDE DF BUILDING TO INSTALLAIION BUILDING

LOCATION
310112.1500058 PLACE BARRIER IN WEASEL PIT OPENING USING BLDG MONORAIL CRANE

310112.1500060 SECURE 1" NUTS WITH PIT MECH SCREH JACK TOOLS

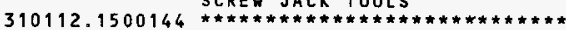
INSTALL HYOROCLONE BARRIER

310112.1500146 ALLOH FOR PACKAGE OF WEASEL PIT GRATING FOR BURIAL INCL $4 \times 4 \times 8$ WOOD BURIAL BOX

310112.1500156 TRANSPORT BARRIER INSIOE OF BUILDING TO INSTALLATION LOCATION

310112.1500158 PLACE BARRIER OVER HYDROCYCLONES USING BLDG CRANE

310112.1500160 DRILL IN ANCHOR BOLTS

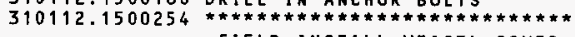
FIELD INSTALL WEASEL COVER

310112.1500256 TRANSPORT BARRIER INSIDE OF BUILDING TO INSTALLATION LOCATION

310112.1500258 PLACE BARRIER OVER PIT 310112.1500260 ALLOWANCE FOR SAFETY HAND RAIL AT HEASEL PIT

310112.1500262 ALLOWANCE FOR WEASEL PIT SST ENCLOSURE ABOVE GRATING

310112.1500264 ALLOWANCE FOR WEASEL PIT SST ENCLOSURE HEPA FILTER

SUBTOTAL MECHANICAL

$\begin{array}{ll}\text { PAGE } & 32 \\ \text { DAIE } & 07 / 24 / 96 \quad 11: 28: 51\end{array}$

$\begin{array}{ll}\text { DAIE } & 07 / 24 / 96 \quad 11: 28: 51 \\ \text { BY } & \text { JPM SMF DKH LGH }\end{array}$

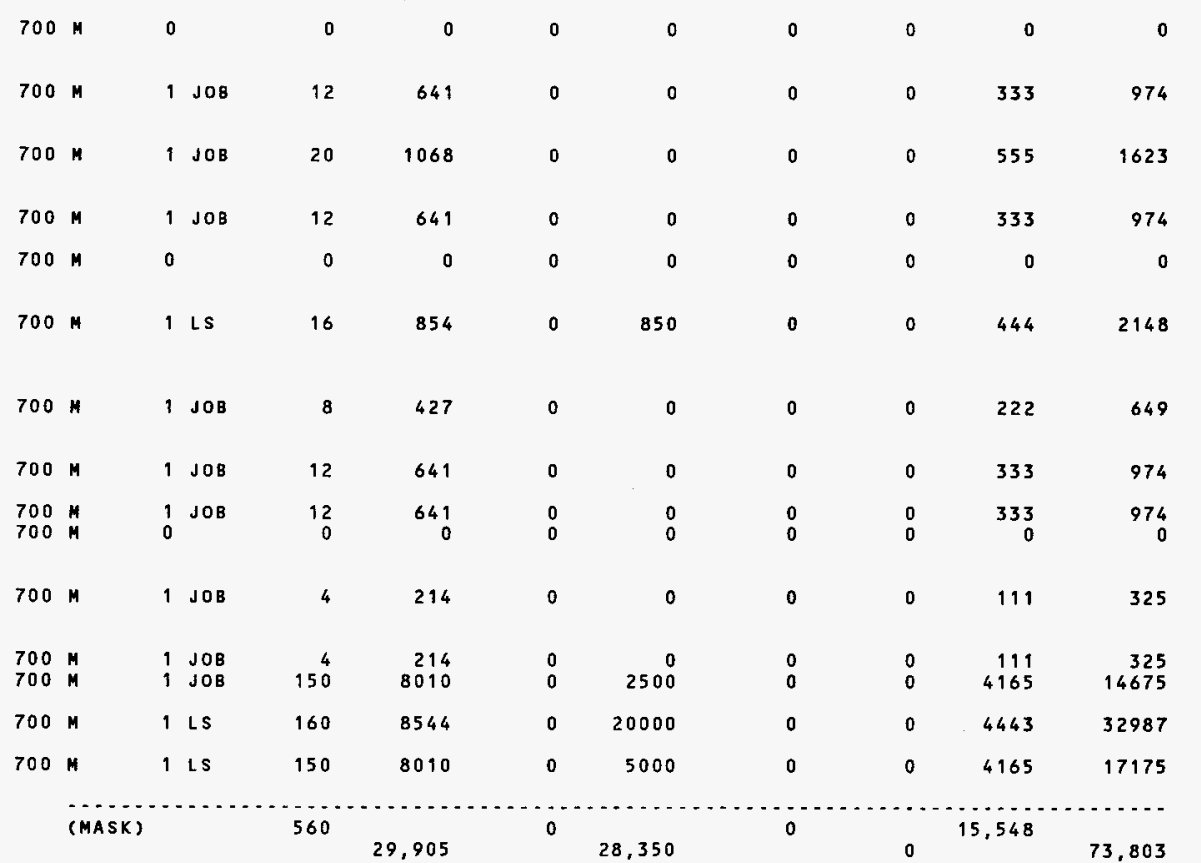

$6-4.5$ 
WHC-SD-SNF-RPT-012, Rev. 0

ICF KAISER HANFORD

WESTINGHOUSE HANF ORD COMPANY JOB NO. E $57991 / 2409$

FILE NO. 2409 PAA3
* * IEST - INTERACIIVE ESTIMATING * *

K-BASINS INTEGRATED WATER SYSTEM PRELIMINARY

DOE ROB - ESTIMATE DETAIL BY HBS / COST CODE
PAGE 33

DATE $07 / 24 / 96 \quad 11: 28: 51$

BY JPM SMF DKH LGH
ACCOUNT
NUMBER DESCRIPIION

SUP $100.00 \%$

GENERAL FOREMAH $7.00 \%$

CONSUMABLES $6.00 \%$

SALES TAX $8.00 \%$

HAREHOUSING $28.00 \%$

OH\&P (ON MARKUPS OHLY)

TOTAL

COSI CODE 70015

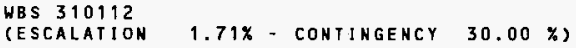

310112.15 MECHAN ICAL

310112.1500004

SHOP FABRICATE HEASEL PIT BARRIER

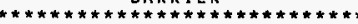

$310112.15000061-1 / 2 " \times 1-1 / 2 "$ C/S ANGLE

$310112.15000081-1 / 2 " C / S$ CHANNEL

$310112.15000101 / 2 \times 1 / 4 " C / S$ FLAT BAR

$310112.15000121 "$ C/S PLATE

$310112.15000141 / 2 "$ C/S PLATE

310112.1500016 I" DIA NELSON STUDS

$310112.15000181 "$ NUT \& WASHER

$310112.15000201 / 2 "$ SQ NEPORENE GSKT MT'L

310112.1500022 WELD 1" DIA NELSON STUDS TO I" PLATE

310112.1500024 WELD $1 / 2 \times 1 / 4$ FLAT TO $1 "$ " $1 / 2 "$ " PLATE (GSKT HOUSING)

310112.1500026 INSERT GSKT

310112.150002811 C/S NUTS \& GSKTS

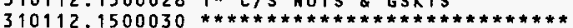
PAINT $C / S$

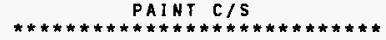

310112.1500032 FIRST COAT FLAT SURFACE C/S 310112.1500034 SECOND COAT FLAT SURFACE C/S 310112.1500040 FIRST COAT STRUCTURAL C/S

310112.1500042 SECOND COAT STRUCTURAL C/S

310112.1500044 TRANSPORT BARRIER FROM SHOPS S $700 \mathrm{~s}$

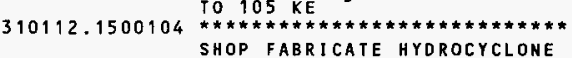
AHD PIPE SHIELD BARRIERS

$30.00 \%$

$700 \mathrm{~s} \quad 0$

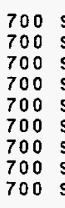

$700 \mathrm{~s}$

$700 \mathrm{~s}$

$700 \mathrm{~s}$

12

$700 \mathrm{~s}$

0
CODE QUANTITY MANHOURS LABOR USAGE

USAGE \begin{tabular}{llll} 
SUB- & EQUIP- & OH\&P & TOTAL. \\
ONTRACT & MENT & \multicolumn{1}{c}{ B \& I DOLLARS }
\end{tabular} \begin{tabular}{llll} 
SUB- & EQUIP- & OH\&P & TOTAL \\
ONTRACT & MENT & \multicolumn{1}{c}{ B $\&$ I DOLLARS }
\end{tabular}

$\begin{array}{rr}560 & 29005 \\ 78 & 4186\end{array}$

1701
2404

2404
8414

4186
1701

D 2404

8414

1,198

63,996

40,869

33,275

17727

0

0

0

0

138,141

31 LB

28 LB
62 LB

3075 LB

12 EA

12 EA

12 EA
75 LF

12 EA

1
30
15

6
6
12
08
158
6
3
1
12

320

320

16447

16447

320
160

160

53

72 LF

1922

$4 J 08$

12 EA

107

107
160
0

$700 \mathrm{~s} \quad 600 \mathrm{SF}$

$700 \mathrm{~s} \quad 600 \mathrm{sF}$

$700 \mathrm{~S} \quad 1 \mathrm{LS}$

$700 \mathrm{~s} \quad 1 \mathrm{LS}$

1 J

36

2
3
0

64

634

951

713

317

o
166
166

333

8552

4387

166
83

28

333

999

56
83
0

517
514
1036
28074
14399
1146
279
119
974
2921
163
279
0

330

495

309

165

0
0

1014

1486

1102

482 


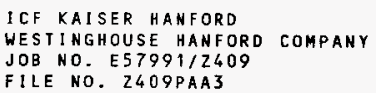

\section{ACCOUNT}

NUMBER

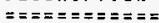

* * IEST - interactive ESTIMATING * *

K-BASINS INTEGRATED HATER SYSTEM

PRELIMINARY

DOE_ROS - ESTIMATE DETAIL BY WBS / COST CODE
PAGE 34

DATE $\quad 07 / 24 / 96 \quad 11: 28: 51$

BY JPM SMF DKH LGH

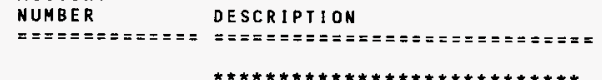

$310112.15001064 \| X 411 \times 1 / 211$ C $/ S$ ANGLE

$310112.150011211 \mathrm{C} / \mathrm{S}$ PLATE

$310112.15001161 / 2 "$ CONCRETE AHCHOR BOLTS

310112.1500124 WELD 4" ANGLE AND q" PLATE

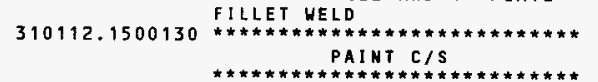

310112.1500132 FIRST COAT FLAT SURFACE C/S

310112.1500134 SECOND COAT FLAT SURFACE C/S

310112.1500140 FIRST COAT STRUCTURAL C/S

310112.1500142 SECOND COAT STRUCTURAL C/S

310112.1500144 TRANSPORT BARRIER FROM SHOPS

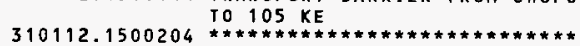

SHOP FABRICATE WEASEL PIT CIS GRATING COVER

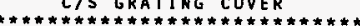

$310112.15002141 / 2 "$ C/S PLATE

$310112.1500230 * * * * * \hbar * \hbar * \hbar * * * * * * * * * * * * * * * * * *$ PAINT C/S

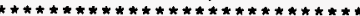

310112.1500232 FIRST COAT FLAT SURFACE C/S 310112.1500234 SECOND COAT FLAT SURFACE C/S 310112.1500244 TRANSPORT BARRIER FROM SHOPS TO $105 \mathrm{KE}$

\section{SUBTOTAL MECHANICAL}

GENERAL FOREMAN $7.00 \%$

CONSUMABLES $6.00 \%$

SALES TAX $8.00 \%$

WAREHOUSING $28.00 \%$

OH\&P (ON MARKUPS ONLY)

TOTAL COST COOE 70015

WBS 310112

CESCALATION

$1.71 \%-\operatorname{CONTINGENCY}$
COSI

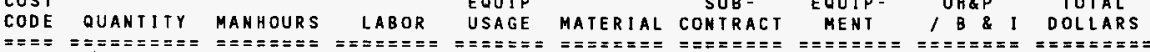
MATERIAL CONTRAC

EQUIP - OH\&P

OH\&P, TOTAL

\begin{tabular}{|c|c|c|c|c|c|c|c|c|c|c|c|}
\hline $\begin{array}{l}700 \\
700 \\
700 \\
700\end{array}$ & $\begin{array}{l}\mathbf{S} \\
\mathbf{S} \\
\mathbf{S} \\
\mathbf{S}\end{array}$ & $\begin{array}{r}600 \\
6755 \\
12 \\
170\end{array}$ & $\begin{array}{l}\text { LB } \\
\text { LB } \\
\text { EA } \\
\text { LF }\end{array}$ & $\begin{array}{r}120 \\
1351 \\
1 \\
85\end{array}$ & $\begin{array}{r}6408 \\
72143 \\
53 \\
4539\end{array}$ & $\begin{array}{l}0 \\
0 \\
0 \\
0\end{array}$ & $\begin{array}{r}600 \\
6755 \\
60 \\
0\end{array}$ & $\begin{array}{l}0 \\
0 \\
0 \\
0\end{array}$ & $\begin{array}{l}0 \\
0 \\
0 \\
0\end{array}$ & $\begin{array}{r}3332 \\
37514 \\
28 \\
2360\end{array}$ & $\begin{array}{r}10340 \\
116412 \\
141 \\
6899\end{array}$ \\
\hline 700 & $s$ & 0 & & 0 & 0 & 0 & 0 & 0 & 0 & 0 & 0 \\
\hline $\begin{array}{l}700 \\
700 \\
700 \\
700 \\
700\end{array}$ & $\begin{array}{l}\text { s } \\
\text { s } \\
\text { s } \\
\text { s } \\
\text { s }\end{array}$ & $\begin{array}{r}300 \\
300 \\
1 \\
1 \\
1\end{array}$ & $\begin{array}{l}\text { SF } \\
\text { SF } \\
\text { IS } \\
\text { LS } \\
\text { JOB }\end{array}$ & $\begin{array}{r}8 \\
12 \\
10 \\
12 \\
6\end{array}$ & $\begin{array}{l}317 \\
475 \\
396 \\
475 \\
238\end{array}$ & $\begin{array}{l}0 \\
0 \\
0 \\
0 \\
0\end{array}$ & $\begin{array}{r}25 \\
20 \\
10 \\
12 \\
0\end{array}$ & $\begin{array}{l}0 \\
0 \\
0 \\
0 \\
0\end{array}$ & $\begin{array}{l}0 \\
0 \\
0 \\
0 \\
0\end{array}$ & $\begin{array}{l}165 \\
247 \\
206 \\
247 \\
124\end{array}$ & $\begin{array}{l}507 \\
742 \\
612 \\
734 \\
362\end{array}$ \\
\hline 700 & s & 0 & & 0 & 0 & 0 & 0 & 0 & 0 & 0 & 0 \\
\hline $\begin{array}{l}700 \\
700\end{array}$ & $\begin{array}{l}S \\
s\end{array}$ & $\begin{array}{r}5712 \\
0\end{array}$ & L8 & $\begin{array}{r}571 \\
0\end{array}$ & $\begin{array}{r}30491 \\
0\end{array}$ & $\begin{array}{l}0 \\
0\end{array}$ & $\begin{array}{r}5712 \\
0\end{array}$ & $\begin{array}{l}0 \\
0\end{array}$ & $\begin{array}{l}0 \\
0\end{array}$ & $\begin{array}{r}15855 \\
0\end{array}$ & $\begin{array}{r}52058 \\
0\end{array}$ \\
\hline $\begin{array}{l}700 \\
700 \\
700\end{array}$ & $\begin{array}{l}5 \\
S \\
S\end{array}$ & $\begin{array}{r}545 \\
545 \\
1\end{array}$ & $\begin{array}{l}\text { SF } \\
\text { SF } \\
\text { JOB }\end{array}$ & $\begin{array}{r}15 \\
22 \\
2\end{array}$ & $\begin{array}{r}594 \\
872 \\
79\end{array}$ & $\begin{array}{l}0 \\
0 \\
0\end{array}$ & $\begin{array}{r}45 \\
37 \\
0\end{array}$ & $\begin{array}{l}0 \\
0 \\
0\end{array}$ & $\begin{array}{l}0 \\
0 \\
0\end{array}$ & $\begin{array}{r}309 \\
453 \\
41\end{array}$ & $\begin{array}{r}948 \\
1362 \\
120\end{array}$ \\
\hline
\end{tabular}

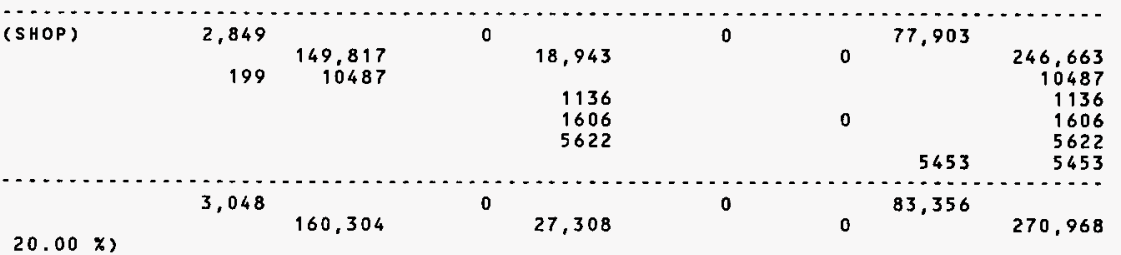

4,246

224.300

0

68,177

0

(1)

16,632

409,110 
WHC-SD-SNF-RPT-012, Rev. 0

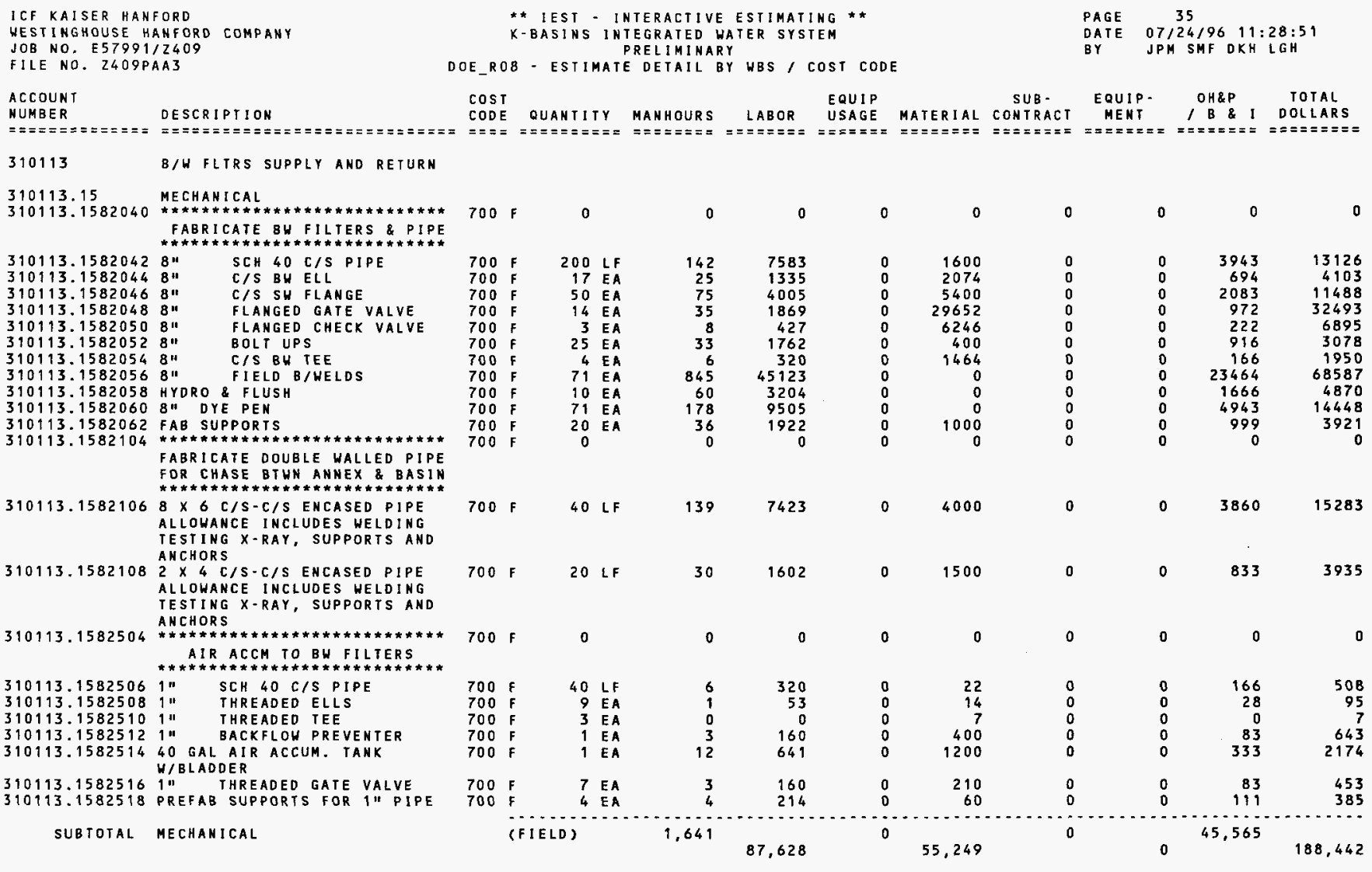


ICF KAISER HANFORD

WESTINGHOUSE HANFORO COMPANY JOB NO. E57991/Z409

FILE NO. Z409PAA3

\section{ACCOUN T \\ NUMBER} DESCRIPTION $==2=\pi==2$

GEMERAL FOREHAH $7.00 \%$

CONSUMABLES $6.00 \%$

SALES TAX $8.00 \%$

WAREHOUSING $28.00 \%$

OH\&P (ON MARKUPS ONLY)

TOTAL

$$
\begin{aligned}
& \text { COST CODE } 70015 \\
& \text { WSS } 310113
\end{aligned}
$$

LESCALATION

$1.71 \%$ - CONTINGENCY $20.00 \%$ )
* * IEST - INTERACIIVE ESTIMATING **

K-BASINS INTEGRATED HATER SYSTEM

PRELIMINARY
DOE ROB - ESTIMATE DETAIL BY WBS / COST CODE $\begin{array}{lll}\text { PAGE } & 36 \\ \text { DATE } & 07 / 24 / 96 & 11: 28: 52\end{array}$

BY JPM SMF DKH LGH

COST EQUIP SUB - EQUIP- OH\&P TOTAL

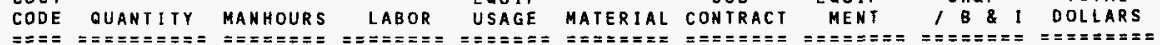
MECHANICAL

310113,15

$310113 \cdot 15.202$ INSTALL BH PIPING

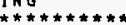

310113.1582218 IHSTALL SUPPORTS

310113.1582604 1 INSTALL PIPING FOR

ACCUM. TANK AT BW FILTERS

$* * * * * * * * * * * * * * * * * * * * * * * * * * * *$

310113.1582606 INSTALL PREFABBED 1" PIPE

310113.158260811 THREADED UNIONS

310113.1582610 HYDRO \& FLUSH

310113.1582612 INSTALL |" SUPPORTS

$310113.1582704 * * * * * * * * * * * * * * * * * * * * * * * * * * * *$ INSTALL DOUBLE HALLEO PIPE 310113.1582706 INSTALL $8 \times 6$ ENCASED PIPE 310113.1582708 INSTALL $2 \times 4$ ENCASED PIPE 310113.1582710 ALLOHANCE FOR DOUBLE WALLED PIPE ENCASEMENT DRAINS

310113.1582712 ALLOWANCE FOR DOUBLE HALLED PIPE EHCASEMENT LEAK DETECTORS

SUBTOTAL MECHANICAL

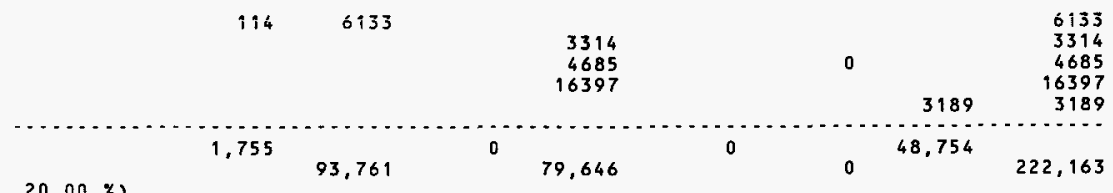

\begin{tabular}{|c|c|c|c|}
\hline 700 & $W$ & 0 & \\
\hline $\begin{array}{l}700 \\
700 \\
700 \\
700 \\
700 \\
700 \\
700 \\
700\end{array}$ & $\begin{array}{l}W \\
W \\
W \\
W \\
W \\
W \\
W \\
W\end{array}$ & $\begin{array}{r}200 \\
11 \\
25 \\
4 \\
200 \\
11 \\
20 \\
0\end{array}$ & $\begin{array}{l}\text { LF } \\
\text { EA } \\
\text { EA } \\
\text { EA } \\
\text { LF } \\
\text { EA } \\
\text { EA }\end{array}$ \\
\hline
\end{tabular}

$\begin{array}{rr}0 & 0 \\ & \\ 142 & 7583 \\ 131 & 6995 \\ 33 & 1762 \\ 128 & 6835 \\ 8 & 427 \\ 31 & 1655 \\ 20 & 1068 \\ 0 & 0\end{array}$

$700 \mathrm{H}$

$700 \mathrm{~W}$

$700 \mathrm{~W}$

$700 \mathrm{H}$

$700 \mathrm{~W}$

$\left(\frac{100}{20}\right.$

$\begin{array}{rl}40 & \text { LF } \\ 5 & E A \\ 3 & E A \\ 4 & E A \\ 0 & \end{array}$

$\begin{array}{rr}6 & 320 \\ 0 & 0 \\ 15 & 801 \\ 3 & 160 \\ 0 & 0\end{array}$

0
0
0
0
0
0
0
0
0

0
0
0
400
0
0
0
80
0

$700 \mathrm{~W} \quad 40 \mathrm{LF}$

$700 \mathrm{~W} \quad 20 \mathrm{LF}$

3 EA

$82 \quad 4379$

$700 \mathrm{~N}$

$700 W$

$3 E A$

1602
3845

1282

$\mathbf{0}$
0
0
0
0

0
25
15
8
0

0
0
0
0
0

0
0
0
0
0

$$
\begin{array}{r}
166 \\
0 \\
417 \\
83 \\
0
\end{array}
$$

486
25

1233

251
0

(SWP)

725

38,714

0

6,528

0

65,372 
WHC-SD-SNF-RPT-012, Rev. 0

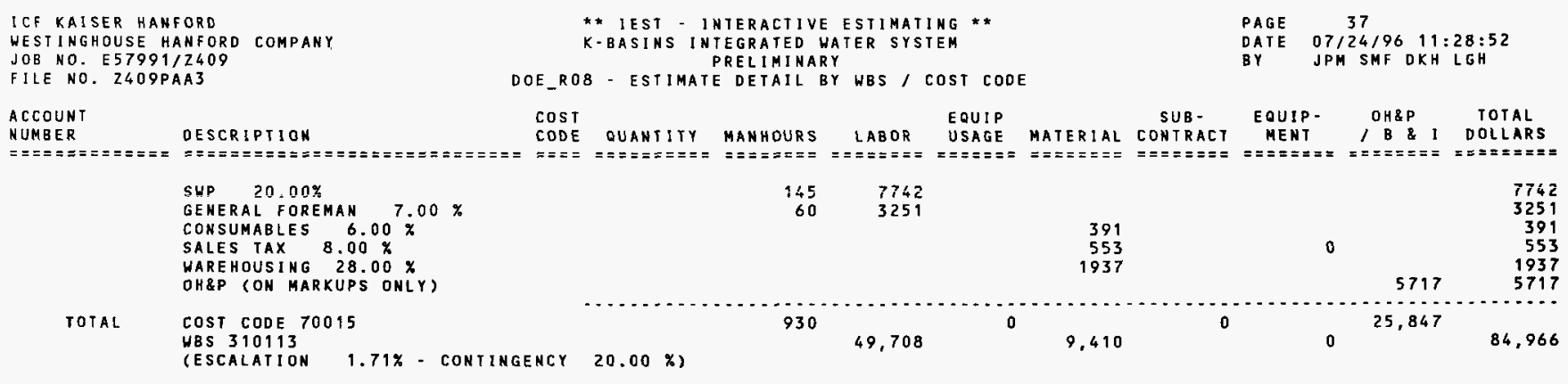

TOTAL WBS 310113 B/W FLTRS SUPPLY AND RETURN

2,686

143,470

89,057

0

74,601

307,130 
ICF KAISER HANFORD

WESTINGHOUSE HANFORD COMPANY JOB NO. E57991/2409

FILE NO. Z409PAA3
* IEST - INTERACTIVE ESIIMATING * *

K-BASINS INTEGRATED HATER SYSTEM

PRELIMINARY

DOE_RO8 - ESTIMATE DETAIL BY WBS / COST CODE
PAGE $\quad 38$

DATE $07 / 24 / 96 \quad 11: 28: 52$

BY JPM SMF DKH LGH

\begin{tabular}{|c|c|}
\hline $\begin{array}{l}\text { ACCOUNT } \\
\text { NUMBER }\end{array}$ & DESCRIPTION \\
\hline 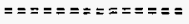 & 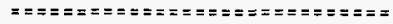 \\
\hline
\end{tabular}

COST EQUIP SUB- EQUTP- OH\&P TOTAL

CODE QUANTITY MANHOURS LABOR USAGE MATERIAL CONTRACT MENT $/$ B \& I DOLLARS

310115

POHER WIRING KE

$310115.16 \quad$ ELECTRICAL

310115.1666000 PRE-ENGINEERED METAL BLDG POHER HIRING

310115.1666801 LIGHTING

310115.1666802 OEVICES

310115.1666803 EQUIPMENT CONNECTIONS

310115.1666804 BASIC MATERIALS

310115.1666805 FIRE ALARM DETECTION

310115.1666806 AIR COMPRESSOR 30 HP CONNECT ALLOHANCE

$310115.16668085 \mathrm{KH} 3 \mathrm{PH}$ Unither, $480 \mathrm{~V}$ ALLOH

310115.1666810 LIGHIING PROTECTION ALL OW

310115.1666812 TELEPHONE/INTERCOM ALLOW

\section{SUBTOTAL ELECTRICAL}

GENERAL FOREMAN $7.00 \%$

CONSUMABLES $6.00 \%$

SALES TAX $8.00 \%$

WAREHOUSING $28.00 \%$

OH\&P (ON MARKUPS ONLY)

TOTAL COST CODE 50316

WBS 310115

(ESCALATION $1.71 \%$ - CONTINGENCY $25.00 \mathrm{x}$ )

$\begin{array}{rrrrrrr}501 & 0 & 0 & 0 & 0 & 0 \\ 501 & 1368 \text { SF } & 52 & 2737 & 0 & 2063 \\ 501 & 1368 \text { SF } & 26 & 1369 & 0 & 213 \\ 501 & 1368 \text { SF } & 26 & 1369 & 0 & 569 \\ 501 & 1368 \text { SF } & 52 & 2737 & 0 & 1245 \\ 501 & 1368 \text { SF } & 26 & 1369 & 0 & 622 \\ 501 & 1 \text { EA } & 26 & 1369 & 0 & 332 \\ 501 & 4 \text { EA } & 68 & 3580 & 0 & 2018 \\ 501 & 1368 \text { SF } & 26 & 1369 & 0 & 534 \\ 501 & 1368 \text { SF } & 26 & 1369 & 0 & 534\end{array}$

0
0
0
0
0
0
0
0
0

0

o

$\begin{array}{ccc}328 & & 0 \\ 22 & 17,268 & 8,130 \\ 1208 & 487 \\ & & 689 \\ & & 2412\end{array}$

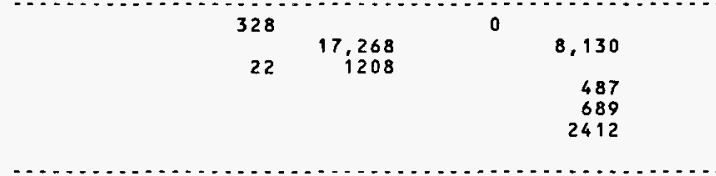

0

8,130

0

1423
712

712
712

1423

712

2650

5405

2703
2413

350

18,476

0

11,720

1862

7460

2615

2615

310115.16 ELECTRICAL 310115.1666000
PRE-ENGINEERED MEIAL BLDG
POHER HIRING

$310 \uparrow 15.1666800$ SERVICE AND DISTRIBUTION

SUBTOTAL ELECTRICAL

SWP $20.00 \%$

GENERAL FOREMAN $7.00 \%$

CONSUMABLES $6.00 \%$

SALES TAX $8.00 \%$

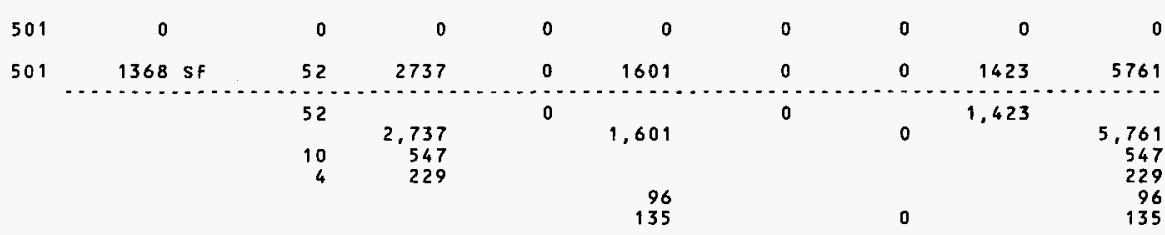

$c-54$ 
WHC-SD-SNF-RPT-012, Rev. 0

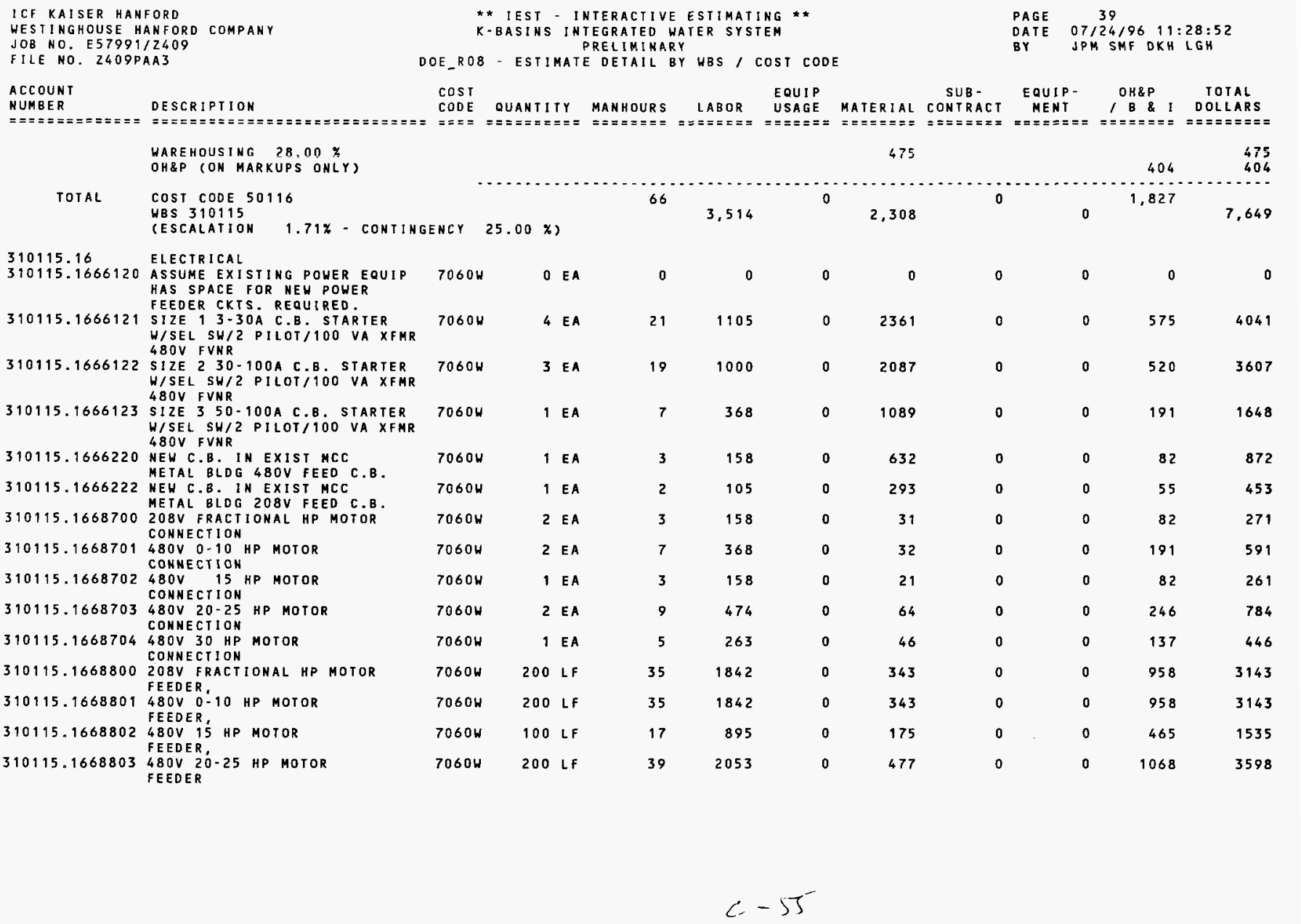


WHC-SD-SNF-RPT-012, Rev. 0

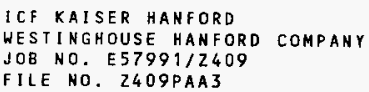

TOTAL

COST CODE 70616

WBS 310115

(ESCALATION $1.71 \%$ - CONTINGENCY
* IEST - INTERACTIVE ESTIMATING *

K-BASINS INTEGRATED WATER SYSIEM PRELIMINARY DOE_RO8 - ESTIMATE DETAIL BY HBS / COST CODE
PAGE 40

DATE $07 / 24 / 96 \quad 11: 28: 52$

BY JPM SMF DKH LGH

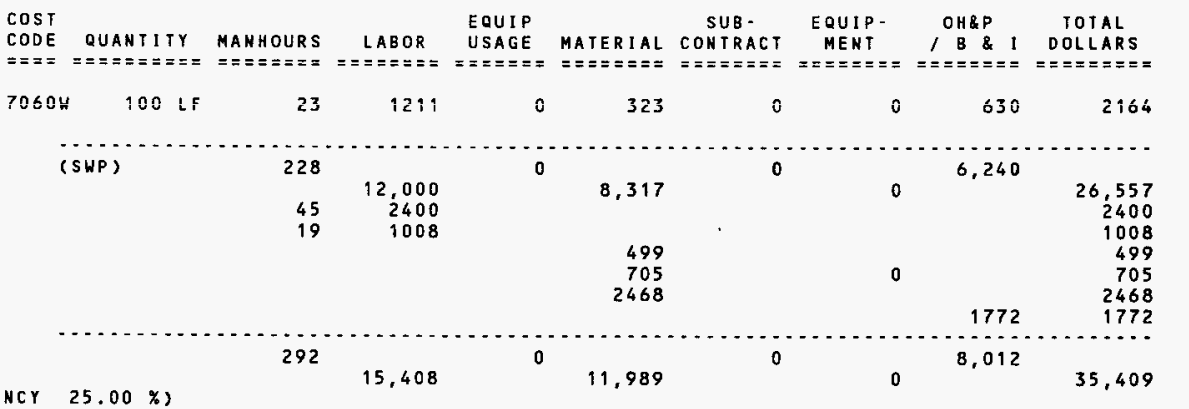

IOTAL WBS 310115 POWER WIRING

KE

710

37,399

0

26,017

o

19,447

82,864 
WHC-SD-SNF-RPT-012, Rev. 0

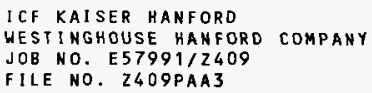

COST EQUIP

COST
CODE QUANTITY MANHOURS LABOR UQUP USA MATERIAL CONTRAC

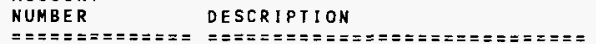

SUB -

PAGE
DATE
OT/24/96 $11: 28: 52$
BY
JPM SMF DKH LGH

EQUIP -

OH\&P TOTAL

HENT $/$ B I DOLLARS
310116 INSTRUMENTATION KE

310116.15 MECHANICAL

310116.1593058 ALLOW FOR GLOV

310116.1593060 MAKE 1" OR LESS SADDLEUELD

CSTL TO EXISTING PIPE

310116.1593062 ALLOW FOR REMOVAL AND PKE DF GLOVE BAG INCL. DRUM FOR BUR!AL

\section{SUBTOTAL MECHANICAL}

SUP $100.00 \%$

GENERAL FOREMAN $7.00 x$

CONSUMABLES $6.00 \%$

SALES TAX $8.00 \%$

WAREHOUSING $28.00 \%$

WH\&P (ON MARKUPS OHLY)

TOTAL COST CODE 70015

WBS 310116

(ESCALATION $1.71 \%$ - CONTINGENCY $30.00 \%$ ) MECHAN I CAL

310116.15

$310116.15930051 / 4 "$ TUBING COPPER

6 EA X 2 LF

310116.1593007 1/4" SHAGELOK MALE CONN

310116.1593019 NOTE: SEE ELECTRICAL FOR INSTRUMENTS HOT SHOWN HERE THREADOLETS ARE SHOWH WITH INSTALLATION WHICH FOLLOWS DPIT

310116.1593025 I

310116.159302711 THREADOLETS, CS FOR CE ETC

310116.1593029 1/4" THREADOLETS, CS

310116.1593032 MAKE 111 OR LESS SADDLEWELD

310116.1593035 CSTL" $1 / 4$ PIPE NIPPLE 1106 C 5 Ti

310116.1593038 1/4" BALL VALVE, BRASS

700

700

$700 \mathrm{~W}$

$700 \mathrm{~W}$

$700 \mathrm{~W}$

$700 \mathrm{H}$

$\begin{array}{rrrrrrrrr}700 & 1 \text { LS } & 8 & 427 & 0 & 250 & 0 & 0 & 222 \\ 700 & 17 \text { EA } & 38 & 2029 & 0 & 17 & 0 & 0 & 1055 \\ 700 & 1 \text { LS } & 4 & 214 & 0 & 110 & 0 & 0 & 111\end{array}$

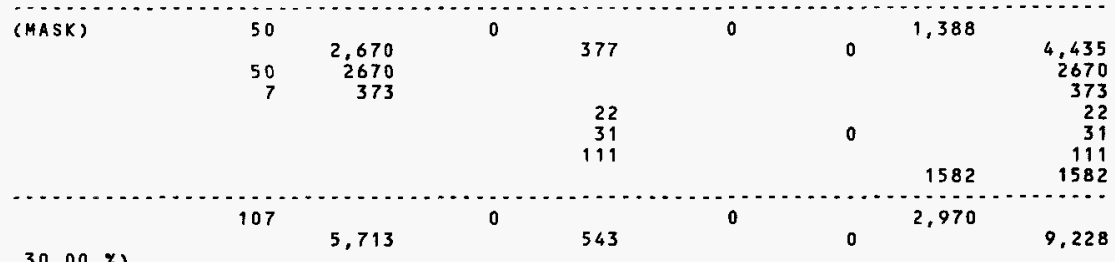

$\begin{array}{ll}700 \mathrm{H} & 12 \text { LF } \\ 700 \mathrm{~W} & 24 \text { EA } \\ 700 \mathrm{~W} & 0\end{array}$

$\begin{array}{rrr}3 & 160 & 0 \\ 9 & 481 & 0 \\ 0 & 0 & 0\end{array}$

6
96
0

0
0
0

2 EA

9 EA

3 EA

$\begin{array}{ll}2 & 107 \\ 5 & 267\end{array}$

55 EA

41 EA

92 EA

52 EA

$\begin{array}{rrrr}2 & 107 & 0 & 700 \\ 5 & 267 & 0 & 630 \\ 1 & 53 & 0 & 15 \\ 4 & 214 & 0 & 275 \\ 92 & 4913 & 0 & 41 \\ 14 & 748 & 0 & 28 \\ 25 & 1335 & 0 & 2600\end{array}$

52 EA
25 1335 
WHC-SD-SNF-RPT-012, Rev . 0

\begin{tabular}{|c|c|c|c|c|c|c|c|c|c|c|c|}
\hline $\begin{array}{l}\text { ICF KAISER HANFORD } \\
\text { HESTINGHOUSE HANFORD } \\
\text { JOB NO. EST991/Z409 } \\
\text { FILE NO. Z409PAA3 }\end{array}$ & $\begin{array}{l}\text { FORD } \\
\text { ANFORD COMPANY } \\
\text { I Z } 409 \\
\text { AA } 3\end{array}$ & $\begin{array}{r}* \\
k \\
O E_{-} R 08\end{array}$ & \multicolumn{4}{|c|}{$\begin{array}{l}\text { ** IEST - INTERACIIVE ESTIMATING * } \\
\text { K-BASINS INTEGRATED HATER SYSIEM } \\
\text { PRELIMINARY } \\
08 \text { - ESTIMATE DETAIL BY WBS / COST CODE }\end{array}$} & & & $\begin{array}{l}\text { PAGE } \\
\text { DAIE } \\
\text { BY }\end{array}$ & \multicolumn{2}{|c|}{$\begin{array}{l}42 \\
07 / 24 / 96 \quad 11: 28: 52 \\
\text { JPM SMF OKH LGH }\end{array}$} \\
\hline $\begin{array}{l}\text { ACCOUNT } \\
\text { NUMBER } \\
===========0\end{array}$ & 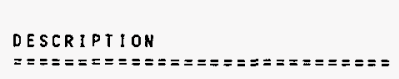 & $\begin{array}{l}\operatorname{COST} \\
\operatorname{CODE} \\
====\end{array}$ & $========$ & $\begin{array}{l}\text { Y MANHOURS } \\
=========\end{array}$ & 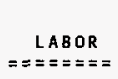 & $\begin{array}{l}\text { EQUIP } \\
\text { USAGE } \\
======\end{array}$ & $=\stackrel{\text { MAIER IAL }}{====== \pm=}$ & $\begin{array}{l}\text { SUB- } \\
\text { CONTRACT } \\
===\approx===\end{array}$ & $\begin{aligned} & \text { EQUIP. } \\
\text { MENT } & =======\end{aligned}$ & $==\begin{array}{l}O H \& P \\
B \& \text { I }\end{array}$ & $\begin{array}{c}\text { TOTAL } \\
\text { DOLLARS } \\
=======\end{array}$ \\
\hline SUBTOTAL & $\begin{array}{l}\text { MECHANICAL } \\
\text { SWP } 100.00 \% \\
\text { GENERAL FOREMAN } 7.00 \% \\
\text { CONSUMABLES } 8.00 \% \\
\text { SALES TAX } 8.00 \% \\
\text { HAREHOUSING } 28.00 \% \\
\text { OH\&P (ON MARKUPS ONLY) }\end{array}$ & & (SWP) & $\begin{array}{r}155 \\
21\end{array}$ & $\begin{array}{r}8,278 \\
8278 \\
1158\end{array}$ & 0 & $\begin{array}{r}263 \\
372 \\
1303\end{array}$ & 0 & c & 4,305 & $\begin{array}{r}16,974 \\
8278 \\
1158 \\
263 \\
372 \\
1303 \\
4907\end{array}$ \\
\hline TOTAL & $\begin{array}{l}\text { COST } \operatorname{COOE} 70015 \\
\text { HBS } 310116 \\
\text { CESCALATION } 1.71 \% \cdot \text { CONTINGE }\end{array}$ & ENCY & $30.00 \%)$ & 331 & 17,714 & 0 & 6,330 & 0 & 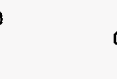 & 9,212 & 33,257 \\
\hline $\begin{array}{l}310116.16 \\
310116.1666000\end{array}$ & $\begin{array}{l}\text { ELECTRICAL } \\
\text { PRE-ENGINEERED METAL BLDG } \\
\text { INSTRUMENTATION }\end{array}$ & $7065 \mathrm{~F}$ & 0 & 0 & 0 & 0 & 0 & 0 & 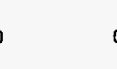 & 0 & 0 \\
\hline 310116.1666001 & $\begin{array}{l}\text { INSTRUMENTATION TOTAL DOLLAR } \\
\text { INCLUDES INSTRUMENTANT AND } \\
\text { ALLOHANCE FOR CND/HIRE/ } \\
\text { TUBING THAT MAY BE REQUIRED }\end{array}$ & $7065 \mathrm{~F}$ & 0 & 0 & 0 & 0 & 0 & 0 & 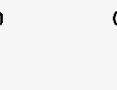 & 0 & 0 \\
\hline 310116.1684500 & $\begin{array}{l}\text { NEH RBC KE } \\
\text { ADO } 8 \text { SLOT RACK REMOTE }\end{array}$ & $7065 \mathrm{~F}$ & $1 E$ & EA & 105 & 0 & 891 & 0 & 0 & 55 & 1051 \\
\hline 310116.1684502 & $\begin{array}{l}\text { NEH RBC KE } \\
\text { ADD POWER SUPPLY }\end{array}$ & $7065 \mathrm{~F}$ & $1 E$ & EA & 105 & 0 & 332 & 0 & 3 & 55 & 492 \\
\hline 310116.1684505 & $\begin{array}{l}\text { NEW RBC KE } \\
\text { ADD AI MODULES }\end{array}$ & $7065 \mathrm{~F}$ & $4 \mathrm{E}$ & EA & 421 & 0 & 780 & 0 & D & 219 & 1420 \\
\hline 310116.1684507 & $\begin{array}{l}\text { NEW RBC KE } \\
\text { ADD DI MODULES }\end{array}$ & $7065 \mathrm{~F}$ & I E & EA & 105 & 0 & 176 & 0 & 3 & 55 & 336 \\
\hline $\begin{array}{l}310116.1684550 \\
310116.168451 \\
310116.1684618\end{array}$ & $\begin{array}{l}\text { CONNECT SIGNALS DI } \\
\text { COHNECT SIGNALS AI } \\
\text { HONDERWARE } \\
\text { ALLOW }\end{array}$ & $\begin{array}{l}7065 \mathrm{~F} \\
7065 \mathrm{~F} \\
7065 \mathrm{~F}\end{array}$ & $\begin{array}{rl}3 & E \\
20 & E \\
1 & E\end{array}$ & $\begin{array}{r}1 \\
10 \\
0\end{array}$ & $\begin{array}{r}53 \\
526 \\
0\end{array}$ & 0 & $\begin{array}{r}1 \\
7 \\
7250\end{array}$ & $\begin{array}{l}0 \\
0 \\
0\end{array}$ & 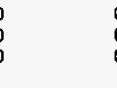 & $\begin{array}{r}28 \\
274 \\
0\end{array}$ & $\begin{array}{r}82 \\
807 \\
7250\end{array}$ \\
\hline $\begin{array}{l}310116.1684900 \\
310116.1684902\end{array}$ & $\begin{array}{l}\text { INSTRUMENT I SAMPLE RACK } \\
\text { SAMPLER / INCLUDES SAMPLE } \\
\text { TUBING }\end{array}$ & $\begin{array}{l}7065 \mathrm{~F} \\
7065 \mathrm{~F}\end{array}$ & $\begin{array}{ll}1 & E \\
7 & E\end{array}$ & $\begin{array}{r}26 \\
226\end{array}$ & $\begin{array}{r}1369 \\
11897\end{array}$ & 0 & $\begin{array}{r}597 \\
34999\end{array}$ & $\begin{array}{l}0 \\
0\end{array}$ & 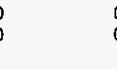 & $\begin{array}{r}712 \\
6186\end{array}$ & $\begin{array}{r}2678 \\
53082\end{array}$ \\
\hline 310116.1684904 & $\begin{array}{l}\text { FQIT } \\
\text { FLOW TOTALIZER/INDICATING } \\
\text { TRANSMITTER }\end{array}$ & $7065 \mathrm{~F}$ & $3 \mathrm{E}$ & 77 & 4053 & 0 & 10512 & 0 & 0 & 2108 & 16673 \\
\hline 310116.1684906 & $\begin{array}{l}\text { FIT } \\
\text { FLOH INDICATING } \\
\text { TRAHSMITTER }\end{array}$ & $7065 \mathrm{~F}$ & $6 \mathrm{E}$ & 154 & 8107 & 0 & 18683 & 0 & D & 4216 & 31006 \\
\hline
\end{tabular}


WHC-SD-SNF-RPT-012, Rev. 0

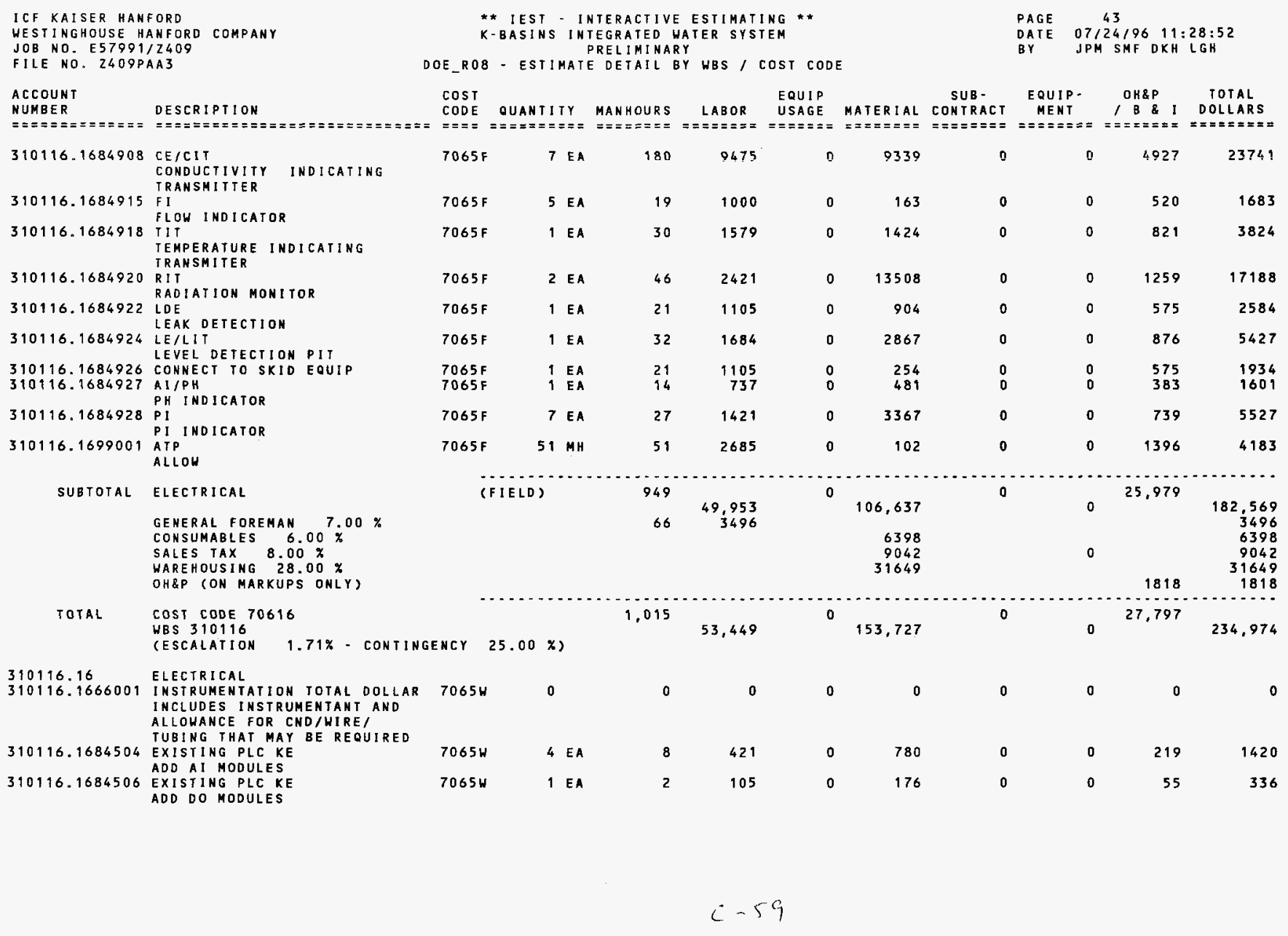


WHC-SD-SNF-RPT-012, Rev. 0

ICF KAISER HANFORD

WESTINGHOUSE HANFORD COMPANY

JOB NO. E57991/2409

FILE NO. 2409PAA3

ACCOUNT
HUMBER
DESCRIPIION

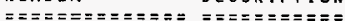

$310116=1684510$ EXIST!HE MM! KE

RELOCATE IN ROOM 7

310116.1684512 NEW SHIFT MGR PERSONAL

WDRK STATIOM

310116.1684514 NEW PLOTTER

310116.1684516 LAN KE TO KH

ALLOW

310116.1684518 HONDERHARE

ALLON

310116.1684550 CONNECT SIGNALS DI

310116.1684551 CONNECT SIGNALS AI

310116.1684552 CONNECT S1GNALS TC

$310116 . \$ 684553$ CONNECT SIGMALS DO

310116.1684904 FOII

FLON TOTALIZER/INDICATING TRANSMITTER

310116.1684906 FIT

FLOW IND I CATING

TRANSNITIER

310116.1684916 PIT

PRESSURE INDICATING

TRANSMITER

310116.1684918 TIT

TEMPERATURE IHDICATING

310116.1684919 TE TRANSMITER

310116.1684922

EMPERATURE DETECTOR

LEAK DETECTION

LE/LIT

LEVEL DETECTION PIT

310116.1684926 CONHECT TO SKID EQUIP 310196.1684930 LI

LEVEL IND I CATOR

310116.1699001 ATP

ALLOH

SUBTOTAL ELECTRICAL

SWP $20.00 \%$

GENERAL FOREMAN $7.00 \%$

CONSUMABLES $6.00 \%$
* * IEST - INIERACTIVE ESTIMATING * *

K-BASINS INTEGRATED WATER SYSTEM

PRELIMINARY

DOE_R08 - ESTIMATE DETAIL BY WBS / COST CODE

COST OUPB - EQUIP. OH\&P TOTAL

CODE QUANTITY MANHOURS LABOR USAGE MATERIAL CONTRACT MENT / B \& I DOLLARS

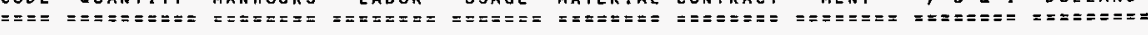

\begin{tabular}{|c|c|c|c|c|c|c|c|c|c|c|}
\hline 70654 & 1 & EA & 22 & 1158 & 0 & 400 & 0 & 0 & 602 & 2160 \\
\hline $7065 \mathrm{H}$ & 1 & EA & 24 & 1263 & 0 & 6170 & 0 & 0 & 657 & 8090 \\
\hline $\begin{array}{l}7065 \mathrm{H} \\
7065 \mathrm{H}\end{array}$ & $\begin{array}{l}1 \\
1\end{array}$ & $\begin{array}{l}E A \\
E A\end{array}$ & 97 & $\begin{array}{r}368 \\
4790\end{array}$ & $\begin{array}{l}0 \\
0\end{array}$ & $\begin{array}{l}3372 \\
5909\end{array}$ & $\begin{array}{l}0 \\
0\end{array}$ & $\begin{array}{l}0 \\
0\end{array}$ & $\begin{array}{r}191 \\
2491\end{array}$ & $\begin{array}{r}3931 \\
13190\end{array}$ \\
\hline $7065 \mathrm{H}$ & 1 & EA & 0 & 0 & 0 & 7250 & 0 & 0 & 0 & 7250 \\
\hline $\begin{array}{l}7065 \mathrm{~W} \\
7065 \mathrm{~W} \\
7065 \mathrm{~W} \\
7065 \mathrm{~W} \\
7065 \mathrm{~W}\end{array}$ & $\begin{array}{r}10 \\
34 \\
2 \\
8 \\
4\end{array}$ & $\begin{array}{l}E A \\
E A \\
E A \\
E A \\
E A\end{array}$ & $\begin{array}{r}5 \\
16 \\
1 \\
4 \\
103\end{array}$ & $\begin{array}{r}263 \\
842 \\
53 \\
211 \\
5422\end{array}$ & $\begin{array}{l}0 \\
0 \\
0 \\
0 \\
0\end{array}$ & $\begin{array}{r}3 \\
11 \\
1 \\
3 \\
14015\end{array}$ & $\begin{array}{l}0 \\
0 \\
0 \\
0 \\
0\end{array}$ & $\begin{array}{l}0 \\
0 \\
0 \\
0 \\
0\end{array}$ & $\begin{array}{r}137 \\
438 \\
28 \\
110 \\
2819\end{array}$ & $\begin{array}{r}403 \\
1291 \\
82 \\
324 \\
22256\end{array}$ \\
\hline $7065 \mathrm{~W}$ & 9 & EA & 231 & 12160 & 0 & 28025 & 0 & 0 & 6323 & 46508 \\
\hline $7065 w$ & 9 & EA & 274 & 14423 & 0 & 13107 & 0 & 0 & 7500 & 35030 \\
\hline $7065 \mathrm{~W}$ & 2 & EA & 61 & 3211 & 0 & 2848 & 0 & 0 & 1670 & 7729 \\
\hline $7065 \mathrm{~W}$ & 2 & EA & 21 & 1105 & 0 & 1029 & 0 & 0 & 575 & 2709 \\
\hline $7065 W$ & 2 & EA & 42 & 2211 & 0 & 1808 & 0 & 0 & 1150 & 5169 \\
\hline $7065 \mathrm{H}$ & 2 & EA & 63 & 3316 & 0 & 5734 & 0 & 0 & 1724 & 10774 \\
\hline $\begin{array}{l}7065 W \\
7065 W\end{array}$ & $\begin{array}{l}1 \\
1\end{array}$ & $\begin{array}{l}\text { EA } \\
\text { EA }\end{array}$ & $\begin{array}{r}21 \\
8\end{array}$ & $\begin{array}{r}1105 \\
421\end{array}$ & $\begin{array}{l}0 \\
0\end{array}$ & $\begin{array}{r}254 \\
2340\end{array}$ & $\begin{array}{l}0 \\
0\end{array}$ & $\begin{array}{l}0 \\
0\end{array}$ & $\begin{array}{l}575 \\
219\end{array}$ & $\begin{array}{l}1934 \\
2980\end{array}$ \\
\hline $7065 \mathrm{~W}$ & 34 & MH & 34 & 1790 & 0 & 68 & 0 & 0 & 931 & 2789 \\
\hline
\end{tabular}

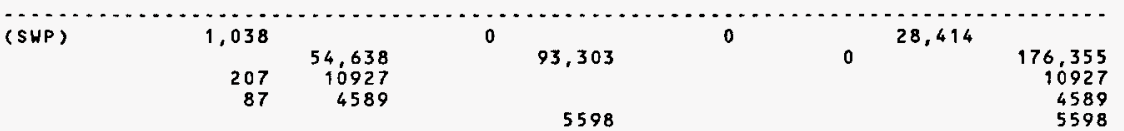


WHC-SD-SNF-RPT-012, Rev. 0

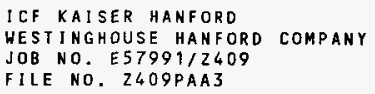

MATERIAL CONTRACT

PAGE 45

DATE $07 / 24 / 96 \quad 11: 28: 53$

BY JPM SMF OKH LGH
SUB -

SUB -

\author{
WBS 310116
(ESCALATION \\ (ESCALATION \\ $1.71 \%-\operatorname{CONTINGENCY}$
}

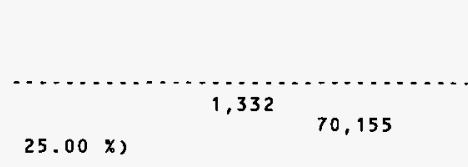

$25.00 \%)$

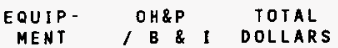

..........

7912
27692 $8068 \quad 27692$

TOTAL WBS 310116 INSTRUMENTATION KE
(

$+$

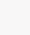

\section{8}

o

241,143
76,463

518,603


WHC-SD-SNF-RPT-012, Rev. 0

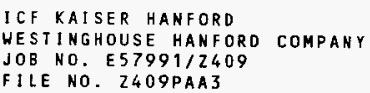

TOTAL

cost CODE 50102

HBS 310117

$1.71 \%$ - CONTINGENCY $20.00 \%$ )
* * IEST - INTERACTIVE ESTIMATING * *

K-BASINS INTEGRATED WATER SYSTEM

$$
\text { PRELIMINARY }
$$

DOE_ROB - ESTIMATE DETAIL BY WBS / COST CODE

\section{PAGE 46 \\ DATE $07 / 24 / 96 \quad 11.28 .53$ \\ BY JPH SMF DKH LGH}

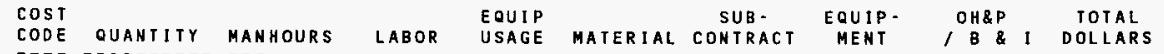

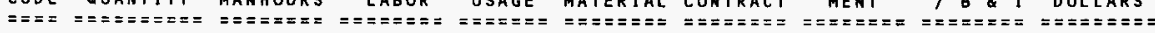

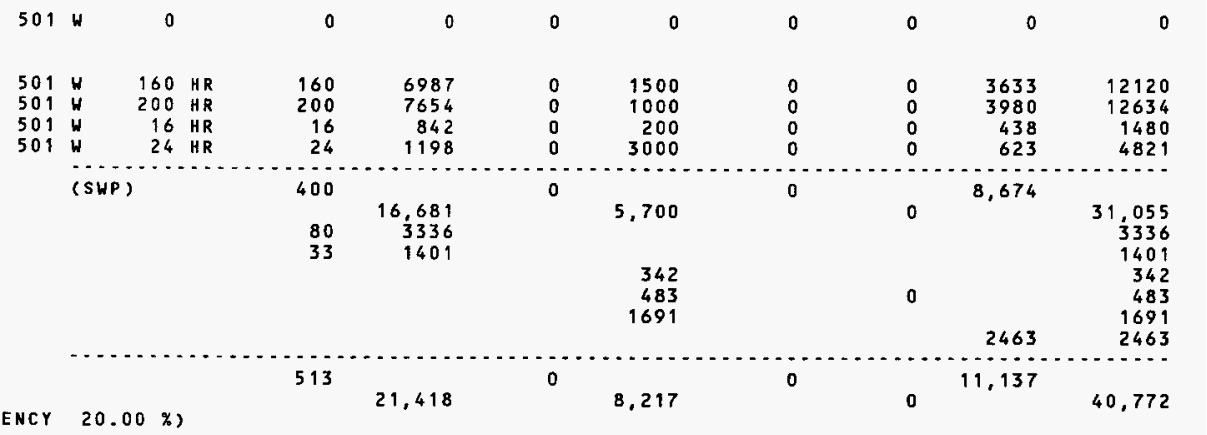

$310117.03 \quad$ CONCRETE

310117.0334501 DRILL REBAR IN EXISTING SLAB

310117.0334502 SCARIFY EXISTIHG CONCRETE

STORAGE SLAB FOR BONDING TO

NEN SLAB (ALLOWANCE)

310117.0334503 CONCRETE BONDING A'GENT

310117.0334504 REMOVE GRN HOUSE AND PKG

FOR BURIAL INCL 2 EA WOOD

BURIAL BOXES ( 3 MEN 3 DAYS)

SUBTOTAL CONCRETE

SWP $100.00 \%$

GENERAL FOREMAN $7.00 \%$

CONSUMABLES $6.00 \%$

SALES TAX $8.00 \%$

WAREHOUSING $28.00 \%$

\begin{tabular}{|c|c|c|c|c|c|c|c|c|c|c|c|}
\hline $\begin{array}{l}501 \\
501\end{array}$ & $M$ & $\begin{array}{r}64 \\
1\end{array}$ & $\begin{array}{l}\text { EA } \\
\text { LS }\end{array}$ & $\begin{array}{l}32 \\
80\end{array}$ & $\begin{array}{l}1554 \\
3884\end{array}$ & $\begin{array}{r}70 \\
150\end{array}$ & $\begin{array}{r}160 \\
0\end{array}$ & $\begin{array}{l}0 \\
0\end{array}$ & $\begin{array}{l}0 \\
0\end{array}$ & $\begin{array}{r}808 \\
2020\end{array}$ & $\begin{array}{l}2592 \\
6054\end{array}$ \\
\hline $\begin{array}{l}501 \\
501\end{array}$ & $M$ & $\begin{array}{l}7 \\
1\end{array}$ & $\begin{array}{l}\text { GAL } \\
\text { JOB }\end{array}$ & $\begin{array}{r}8 \\
72\end{array}$ & $\begin{array}{r}306 \\
2755\end{array}$ & $\begin{array}{l}0 \\
0\end{array}$ & $\begin{array}{r}147 \\
1700\end{array}$ & $\begin{array}{l}0 \\
0\end{array}$ & $\begin{array}{l}0 \\
0\end{array}$ & $\begin{array}{r}159 \\
1433\end{array}$ & $\begin{array}{r}612 \\
5888\end{array}$ \\
\hline & (MASK) & $\cdots$ & & 192 & & 220 & - - & 0 & $\cdots$ & 4,420 & - \\
\hline & & & & $\begin{array}{r}192 \\
26\end{array}$ & $\begin{array}{r}8,499 \\
8499 \\
1189\end{array}$ & & $\begin{array}{r}2,007 \\
120 \\
170 \\
595\end{array}$ & & 0 & 5038 & $\begin{array}{r}15,146 \\
8499 \\
1189 \\
120 \\
170 \\
595 \\
5038\end{array}$ \\
\hline
\end{tabular}


WHC-SD-SNF-RPT-012, Rev. 0

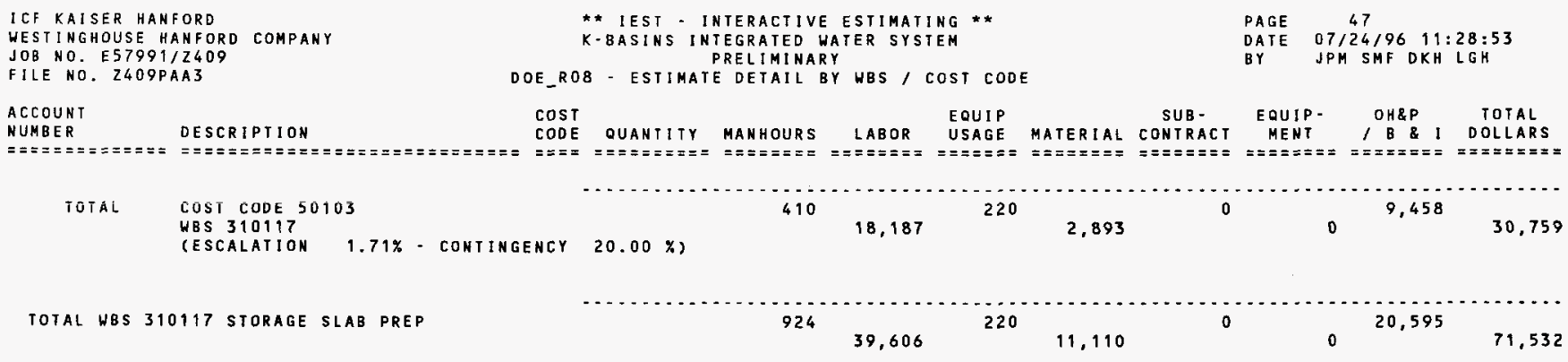

$$
2-63
$$




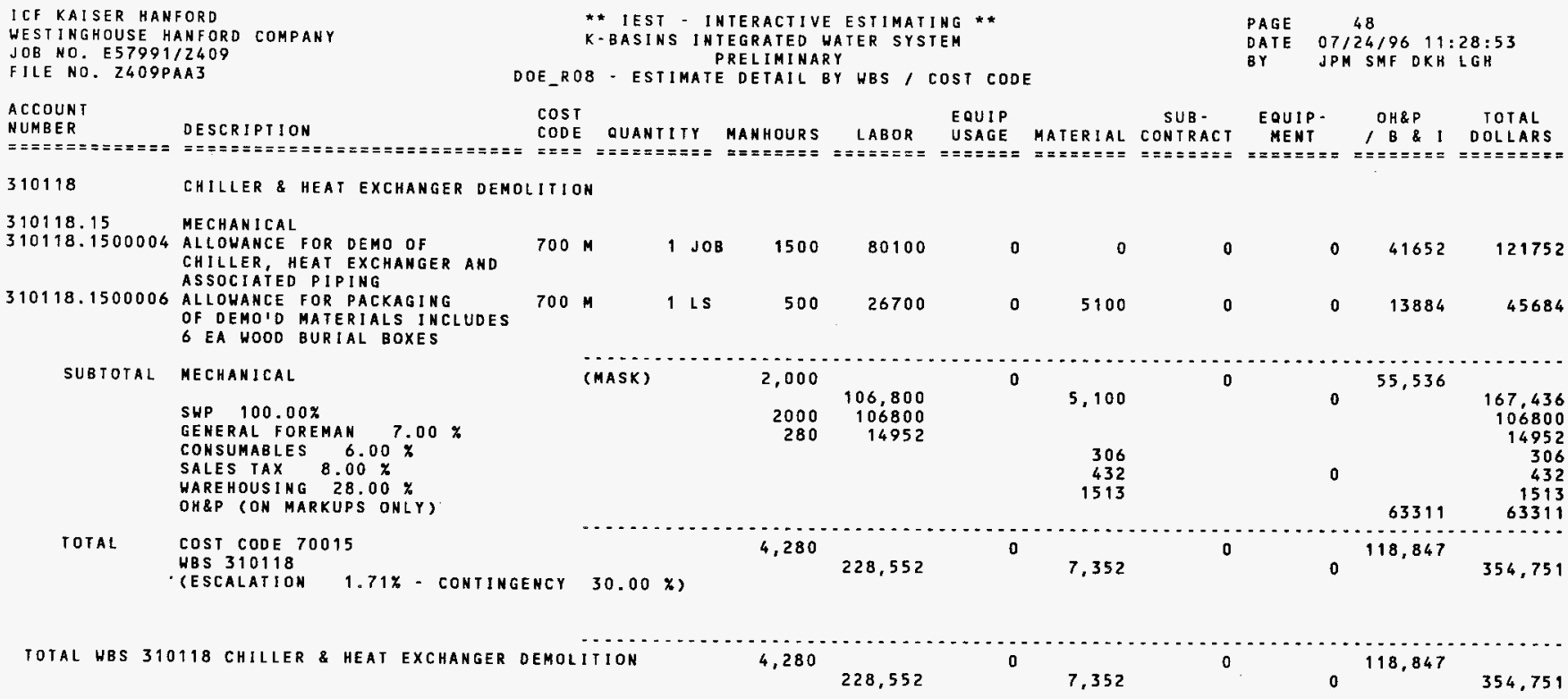


WHC-SD-SNF-RPT-012, Rev. 0

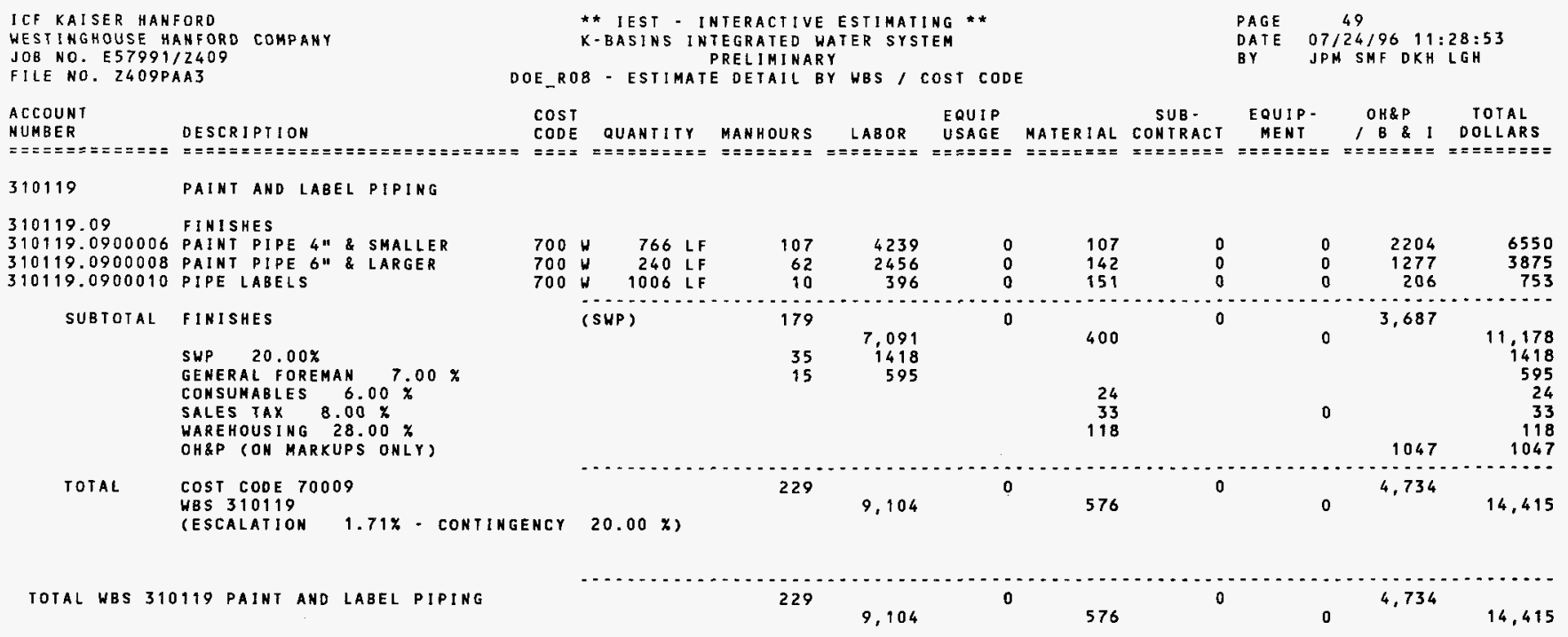




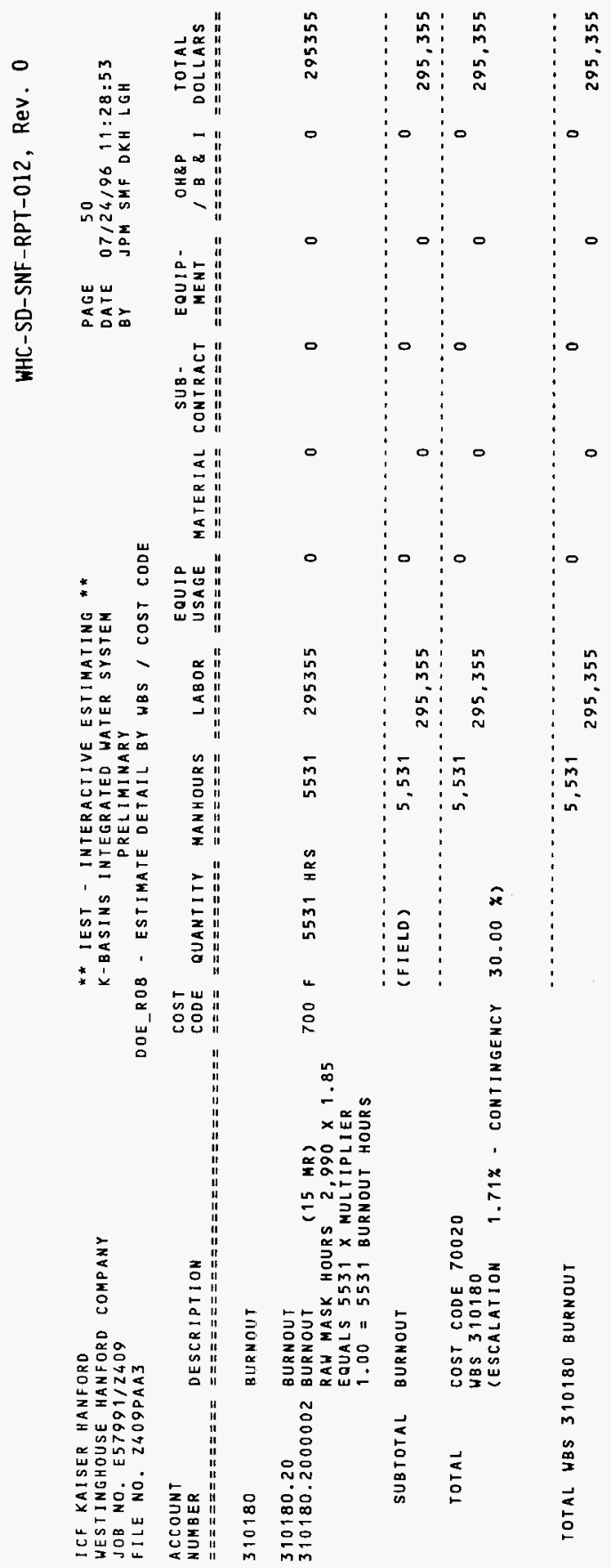


WHC-SD-SNF-RPT-012, Rev. 0

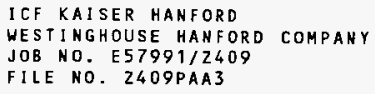

TOTAL

\section{COST CODE 70001}

HBS 310190 CESCALATION

$1.71 \% \cdot \operatorname{CONTINGENCY}$
** IEST - INIERACIIVE ESTIMATING * *

K-BASINS INTEGRATED WATER SYSTEM PRELIMINARY

DOE_RO8 - ESTIMATE DETAIL BY WBS $/$ COST CODE
PAGE
DATE 071
BY TPM SMF DKH LGH

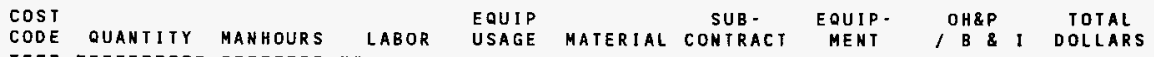

CODE QUANITY MANHOURS LABOR USAGE MATERIAL CONTRACT MENT I B I DOLLARS

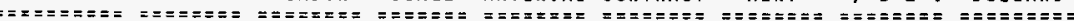

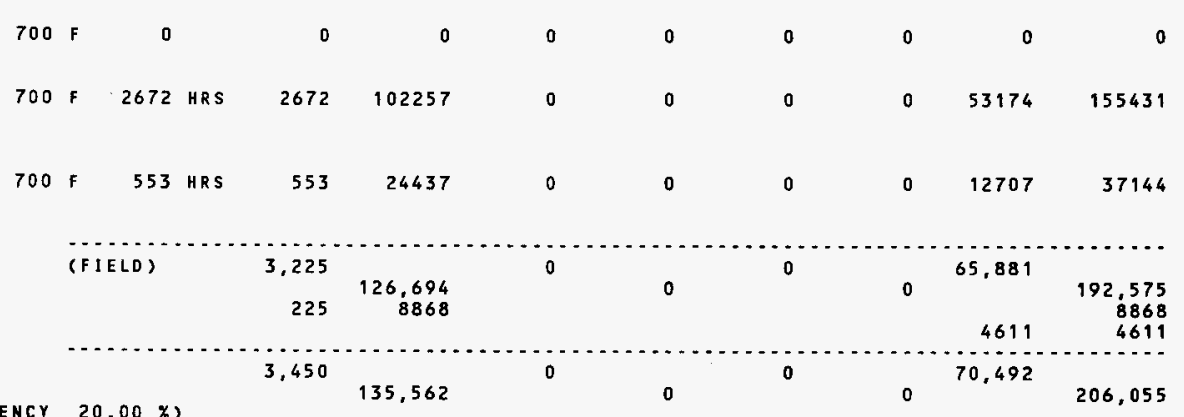

TOTAL WBS 310190 SUPPORT REQUIREMENTS

3,450

135,562

0

0

70,492

206,055 
WHC-SD-SNF-RPT-012, Rev. O

ICF KAISER HANFORD

WESTINGHOUSE HANFORD COMPANY JOB NO. E57991/2409 FILE NO. Z409PAA3

\section{ACCOUNT}

NUMBER

DESCR I PT ION

* * IEST - INTERACTIVE ESTIMATING * *

$K$-BASINS INTEGRATED WATER SYSTEM PRELIMINARY

DOE_ROB - ESTIMATE DETAIL BY WBS / COST CODE
PAGE 52

DATE $07 / 24 / 96 \quad 11: 28: 53$

BY JPM SMF DKH LGH

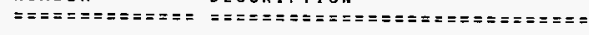

EQUIP SUB - EQUIP - OH\&P TOTAL

CODE QUANTITY MANHOURS LABOR USAGE MATERIAL CONTRACT MENT IB I DOLLARS 310191 NEH RAD STORAGE PAD

$310191.02 \quad$ SITEHORK

310191.0234502 DEMO ASPHALT

310191.0234504 HAUL OFF WASTE

310191.0234506 FENCE 61 HIGH

$310191.02345084^{\prime}$ GATE

$310191.0234510160^{\circ}$ SLIOER GATE

310191.023451281 SWING GATE

SUBTOTAL SITEHORK

GENERAL FOREMAN $7.00 \%$

CONSUMABLES $6.00 \%$

SALES TAX $8.00 \%$

WAREHOUSING $28.00 \%$

OH\&P (ON MARKUPS ONLY)

TOTAL

COST COOE 55002
WBS 310191

UBS 310191

(ESCALATION $1.71 \%$ - CONTINGENCY $20.00 \quad x$ )

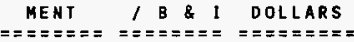

\section{CONCRETE}

310191.0345602 GRADE \& SCREED SOG

310191.0345604 FORM SOG

310191.0345606 KEY JOINTS

310191.0345608 STRIP \& OIL

310191.0345610 CONCRETE SOG

310191.0345612 CURING

310191.0345614 REBAR

310191.0345616 TROWEL FINISH

\section{SUBTOTAL CONCRETE}

GENERAL FOREMAN $7.00 \%$

CONSUMABLES $6.00 \%$

SALES TAX $8.00 \%$

WAREHOUSING $28.00 \%$

OHEP (ON MARKUPS ONLY)

TOTAL COSI CODE 55003

WBS 310191

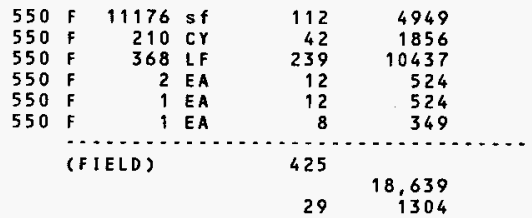

29

454

19,943

4,912

(n)

0,368

7522

965

5427

272

272
181

282

18072

1196

1296

$0 \quad 001810830$

9,690

31,737

1304

304

204

1011 678

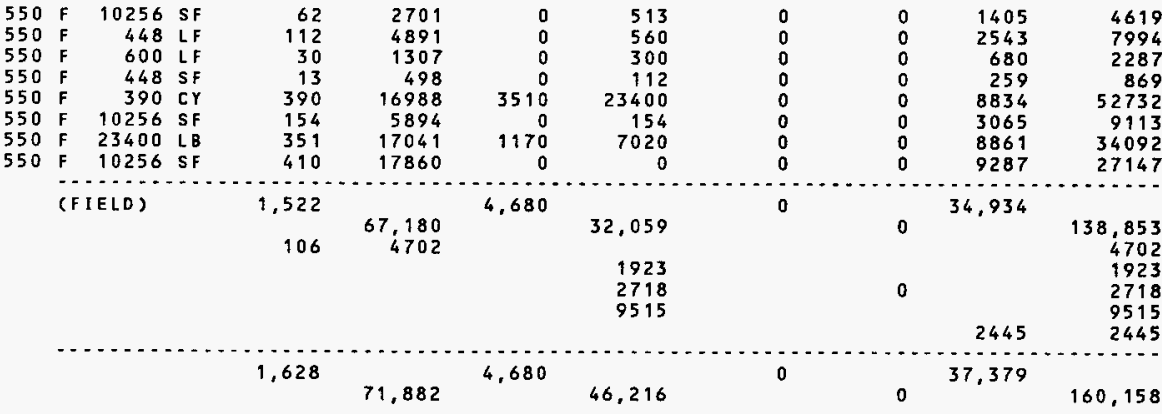


WHC-SD-SNF-RPT-012, Rev. 0

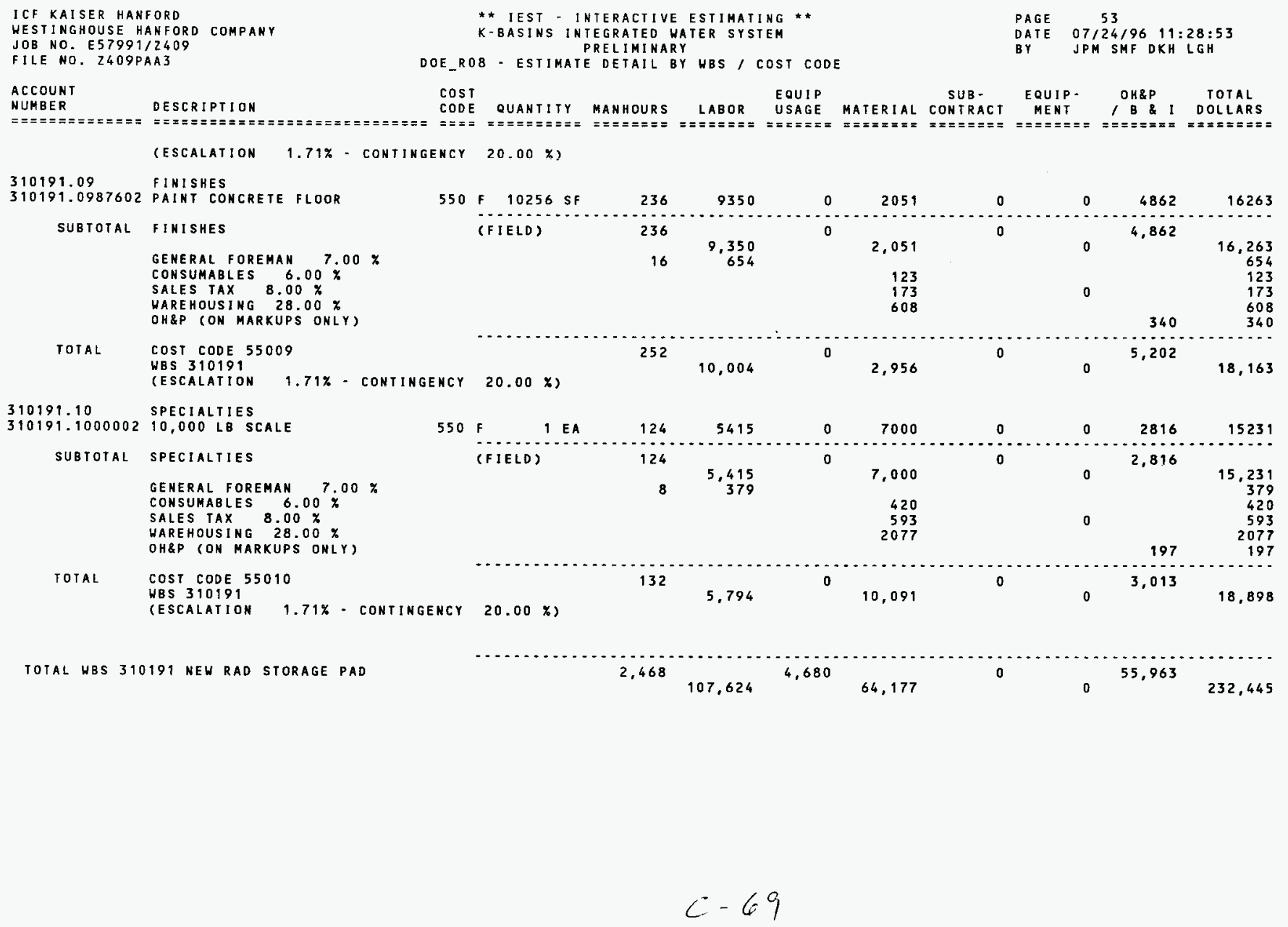


WHC-SD-SNF-RPT-012, Rev. 0

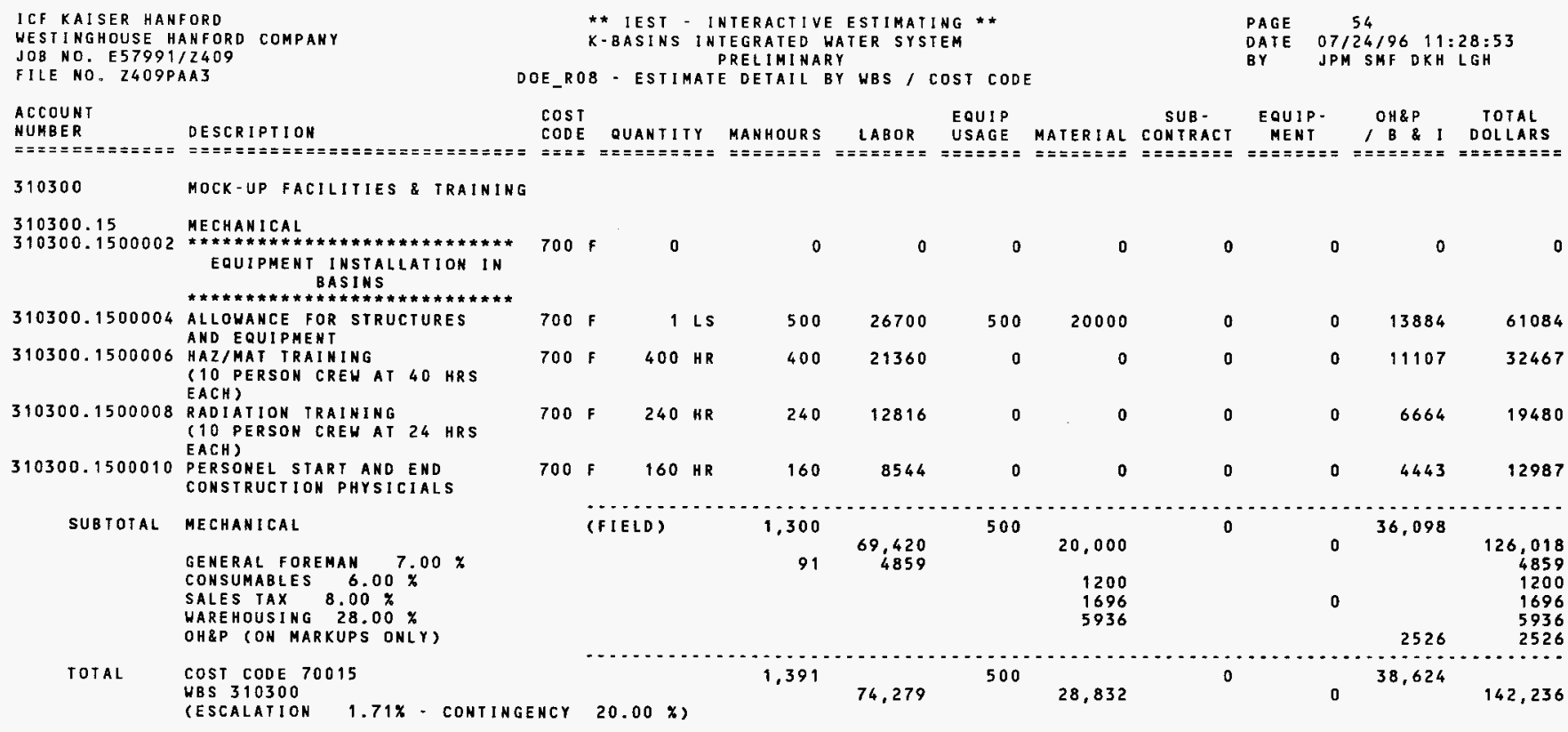

TOTAL WBS 310300 MOCK-UP FACILITIES \& TRAINING

$1,391 \quad 74,279 \quad 500 \quad 28,832$

0

o 38,624

142,236 
WHC-SD-SNF-RPT-012, Rev. 0

I CF KAISER HANFORD

WESTINGHOUSE HAHFORD COMPANY

JOB NO. E $57991 / 2409$

FILE NO. Z409PAA3

DESCRIPTION

* * IEST - INTERACTIVE ESTIMATING * *

K-BASINS INTEGRATED WATER SYSTEM PRELIMINARY

DOE_ROB - ESTIMATE DETAIL BY HBS/ COST CODE

EQU I P

CODE GUATY MANHOURS

USAGE
PAGE 55

DAJE 07/24/96 11:28:54

BY JPM SMF DKH LGH

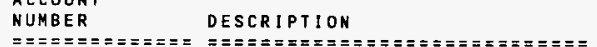

SUB -
MATERIAL CONIRAC

EQUIP -

OH\&P

$\begin{array}{lll}\text { OIP - } & \text { OH\&P } & \text { TOTAL } \\ \text { MENT } & \text { B \& I DOLLARS }\end{array}$

$======== \pm$ NEW RECIRC PUMPS
$310307 \quad$ N

310307.15

MECHANICAL

$310307 . \$ 522004$

FIELD FAB

$700 \mathrm{~F}$

3 EA

PURCHASE NEH RECIC PUMPS

500 GM P-1A, $1 \mathrm{~B} \& 1 \mathrm{C}$

(CONTRARY TO DWGS - AS DI -

RECTED BY BNFL, MARK \& ERIC)

310307.1522007 4" PIPE SCH 40 CS

$310307.15220102 "$ PIPE SCH 40 CS

310307.1522022 4" ELB 90 CS BH SCH 40

310307.1522028 2" ELB 90 CS BW SCH 40

310307.1522048 4" WELDOLET CS (PURCH ONLY) (SEE INSTALL)

310307.15220542 " (SEE INSTALL) (PURCH ONLY)

3103071522060 4" FLANGE HN 150 \# CS

$310307.15220662 "$ "FLANGE WN 150\# CS

310307.1522076 4" GATE VALVE, FLGD CS

310307.15220842 " GATE VALVE, FLGD CS

$310307.15220924 " \mathrm{BH} \cdot \mathrm{S}$

310307.15220972 " BW'S

310307.1522106 4" DYE PEN HELD EXAM

310307.1522110 2" DYE PEM HELD EXAMS

310307.1522116411 PIPE SUPPORT FABRICATION (ASSUMED SIMPLE BASED ON DHG $H-1-34693$ EXAMPLES )

310307.1522120 2" PIPE SUPPORT FABRICATIOH

310307.1522152 INSTALLATION

310307.1522156 INSTALL NEW RECIC PUMPS P- IA, 1 B \& 1C $P-1 A, 1 B \& 1 C$

310307.9522158 BOLTUP 8" FLGS (BOLT SETS \& GSKT)

310307.1522160 INSTALL 4" PIPE SPOOL

310307.1522164 INSTALL 2" PIPE SPOOL

$310307.15221684 "$ BW'S (CS SCH 40)

310307.152217421 BW'S CS SCH 40

310307.15221824 " DYE PEN HELD EXAM

$310307.15221944 "$ PIPE SUPPORTS INSTALL IN

310307.1522202 2" PIPE SUPPORTS INSTALL'N

310307.1522208 HYDRO 4" PIPE SPOOLS 10 LF \& FLUSH

700

700

700

$700 \mathrm{~F}$

$700 \mathrm{~F}$

$700 \mathrm{~F}$

$700 \mathrm{~F}$

$700 \mathrm{~F}$

$700 \mathrm{~F}$

$700 \mathrm{~F}$

$700 \mathrm{~F}$

$700 \mathrm{~F}$

$700 \mathrm{~F}$

$700 \mathrm{~F}$

$700 \mathrm{~F}$

$700 \mathrm{~F}$

$700 \mathrm{~F}$

$700 \mathrm{~F}$

$700 \mathrm{~F}$

$700 \mathrm{~F}$

$700 \quad F$

$700 \mathrm{~F}$

$700 \mathrm{~F}$

700

$700 \mathrm{~F}$

$700 \mathrm{~F}$

$700 \mathrm{~F}$

$700 \mathrm{~F}$ $=======$

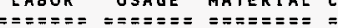

ME
$x=x=x==x=x==x== \pm=$ 


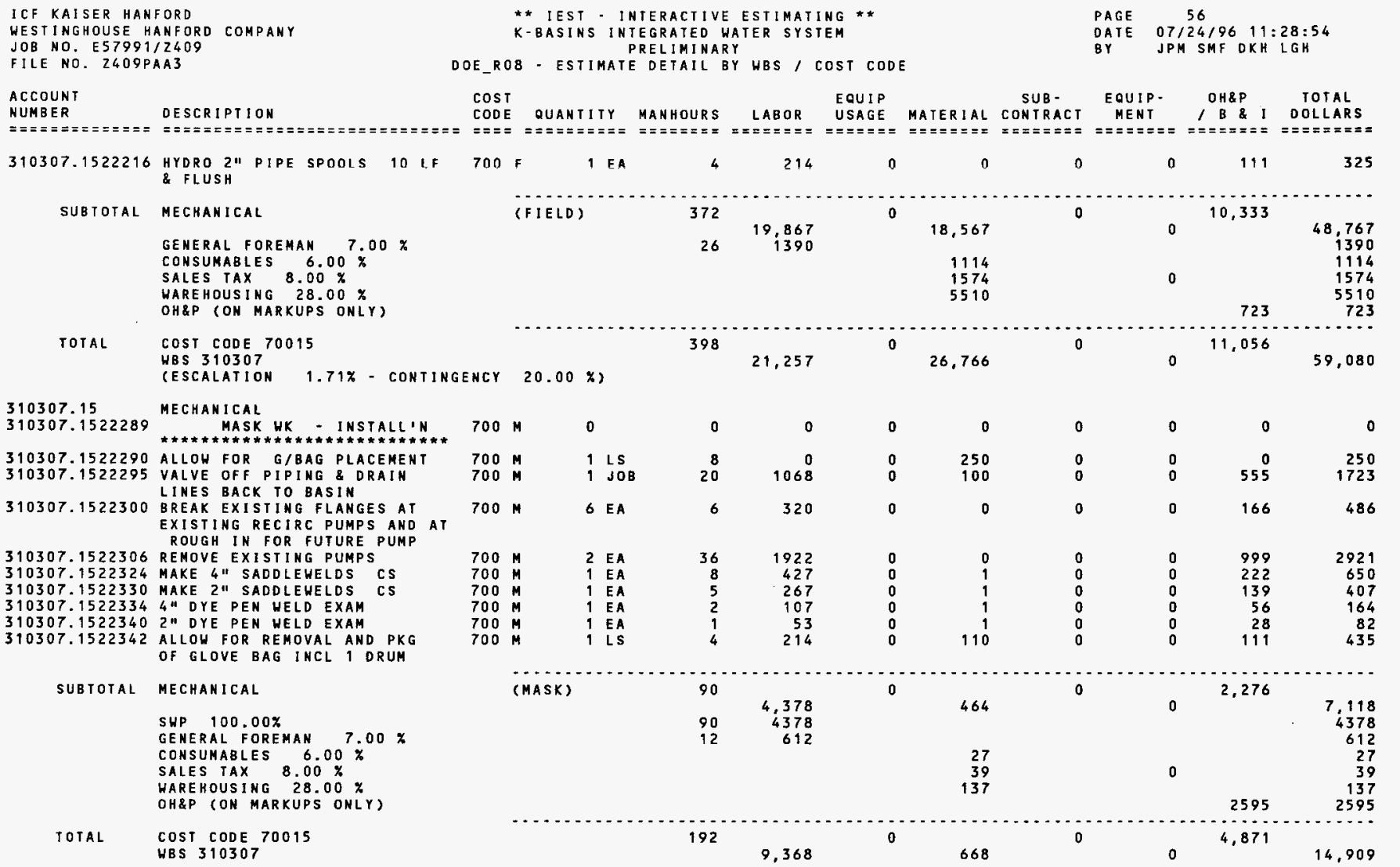


WHC-SD-SNF-RPT-012, Rev. 0

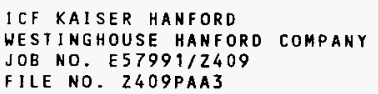

ACCOUNT

NUMBER

DESCRIPTION

= = = = = = = = = = = = = = = = = = = = = = = = =

(ESCALAT!ON

1. 71\% - COnTIMEEUCY

* * Iest - interactive estimating *

K-BASINS INTEGRATED WATER SYSTEM PRELIMINARY

DOE_ROB - ESTIMATE DETAIL BY WBS / COST CODE

\author{
COST
}

CODE QUANTITY MANHOURS LABOR USAGE

EQUIP
USAGE MATERIAL CONTRACT

PAGE 57

DATE $07 / 24 / 96 \quad 11: 28: 54$

BY JPM SMF DKH LGH

EQUIP- OH\&P TOTAL MENT 1 DOLLARS $2.00 \%$
IOTAL WBS 310307 NEW RECIRC PUMPS

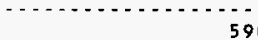

590
30,626
0

27.435

0

15,927 
WHC-SD-SHF-RPT-012, Rev. 0

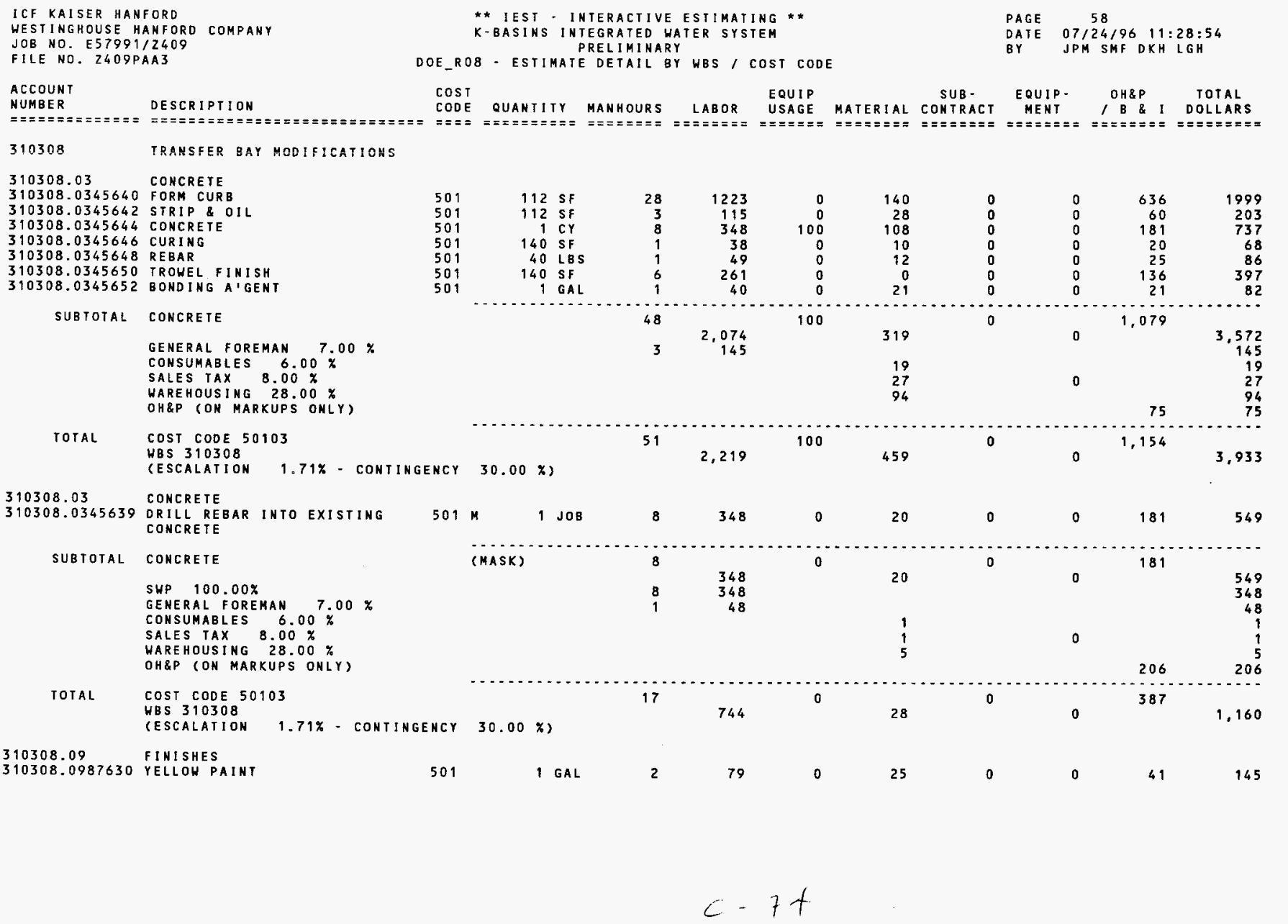


WHC-SD-SNF-RPT-012, Rev. 0

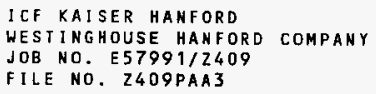

WESTINGHOUSE HANFORD COMPANY

* * IEST - INTERACTIVE ESTIMATING *

K-BASINS INTEGRATED WATER SYSTEM PRELIMINARY

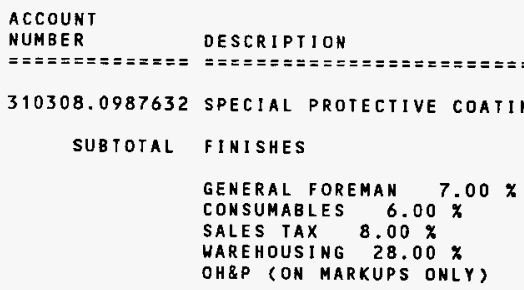

SUBTOTAL FINISHES

GENERAL FOREMAN $7.00 \%$

CONSUMABLES $6.00 \%$

SALES TAX $8.00 \%$

WAREHOUSING $28.00 \times$

OH\&P (ON MARKUPS ONLY)

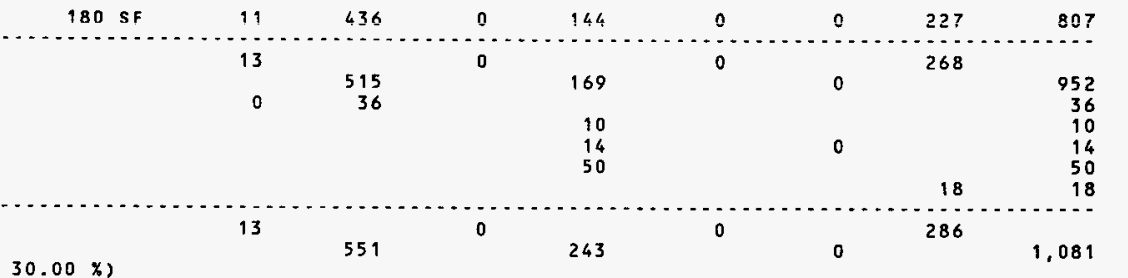

IOTAL

\section{$\cos T \operatorname{CODE} 50109$}

HBS 310308

(ESCALATION $1.71 \%$ - CONTINGENCY $30.00 \%$ )

310308.15

MECHANICAL

310308.1500006 RECEIVE UNLOAD INSPECT AND PREP IXA'S FOR INSTALLATION CONNECT SUPPLY \& RETURN

310308.1500010 CONNECT SUP

310308.1500012 CDNNECT VENT PIPE

310308.1500014 CONNECT DRAIN PIPE

\section{SUBTOTAL MECHANICAL}

GENERAL FOREMAN $7.00 \%$ OH\&P (ON MARKUPS ONLY)

TOTAL

\section{COST CODE 70015}

$\begin{array}{rrrr}700 & 3 \text { EA } & 3 & 107 \\ 700 & 3 \text { EA } & 9 & 322 \\ 700 & 3 \text { EA } & 6 & 215 \\ 700 & 3 \text { EA } & 6 & 215 \\ & & 24 & 859 \\ & & 1 & 60\end{array}$

PAGE 59

DATE $07 / 24 / 96 \quad 11: 28: 54$

BY JPM SMF DKH LGH

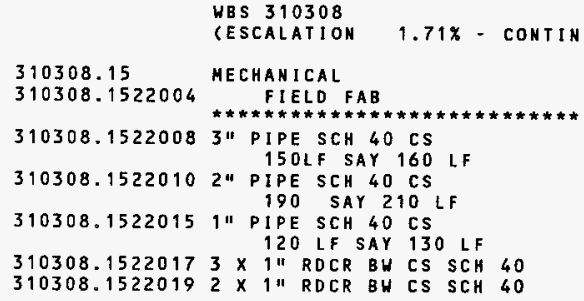

WBS 310308

(ESCALATION

25

919

$\begin{array}{rrrr}700 \mathrm{~F} & 0 \\ 700 \mathrm{~F} & 160 \text { LF } \\ 700 \mathrm{~F} & 210 \text { LF } \\ 700 \mathrm{~F} & 130 \text { LF } \\ 700 \mathrm{~F} & 1 \text { EA } \\ 700 \mathrm{~F} & 1 \text { EA }\end{array}$

0
45
40
18
1
0

919

19

......

0

0

\begin{tabular}{lllrr}
0 & 0 & 0 & 56 & 163 \\
0 & 0 & 0 & 167 & 489 \\
0 & 0 & 0 & 112 & 327 \\
0 & 0 & 0 & 112 & 327 \\
\hdashline 0 & 0 & & 447 & 1,306 \\
& & & & 60
\end{tabular}

478

1,397

$\begin{array}{rrrrrr}0 & 0 & 0 & 0 & 0 & 0 \\ 2403 & 0 & 480 & 0 & 0 & 1250 \\ 2136 & 0 & 552 & 0 & 0 & 1111 \\ 961 & 0 & 72 & 0 & 0 & 500 \\ 53 & 0 & 30 & 0 & 0 & 28 \\ 0 & 0 & 17 & 0 & 0 & 0\end{array}$

4133

3799

1533 
WHC-SD-SNF-RPT-012, Rev. 0

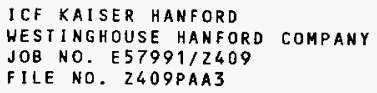

* * iEST - INTERACTIVE ESTIMATING * *

K-BASINS INTEGRATED WATER SYSTEM PRELIMINARY

DOE_ROB - ESTIMATE DETAIL BY WBS/ COST CODE
PAGE 60

DATE $07 / 24 / 96 \quad 11: 28: 54$

BY JPM SMF DKH LGH

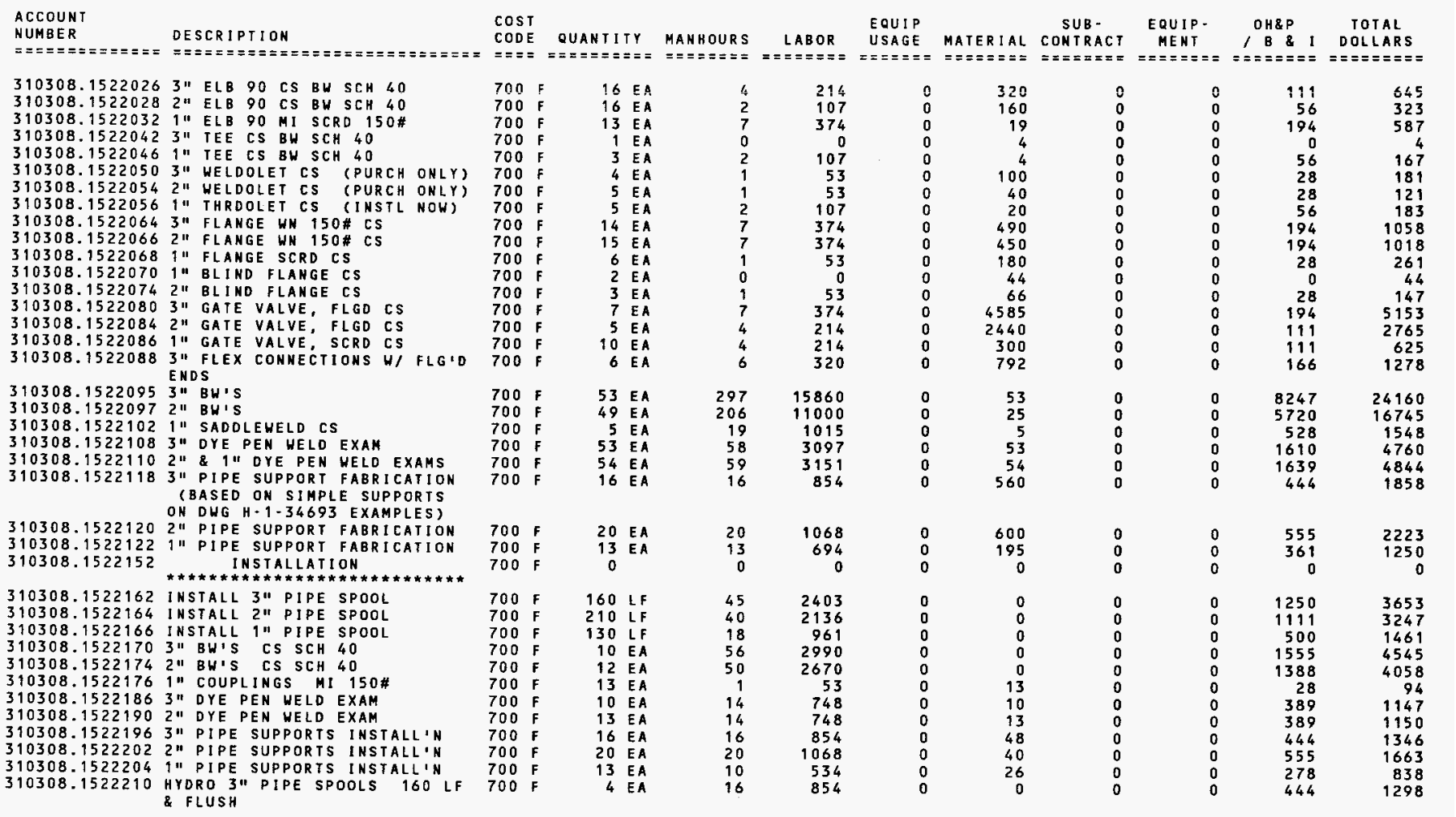


WHC-SD-SNF-RPT-012, Rev. 0

ICF KAISER HANFORD

WESTIHGHOUSE HANFORD COMPANY

JOB NO. E57991/Z409

FILE NO. 2409 PAA3

ACCOUNT

NUMBER

DESCRIPTION

* * IEST - INTERACTIVE ESTIMATING * *

K-BASINS INTEGRAIED WATER SYSTEM PRELIMINARY

= = = = = = = = = = = = = = = = = = = = = = = =

310308.1522216 HYDRO 2" PIPE SPOOLS 190 LF \& FLUSH

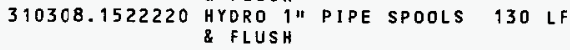

SUBTOTAL MECHANICAL

GENERAL FOREMAN $7.00 \%$ CONSUMABLES $6.00 \%$ SALES TAX B.00 $\boldsymbol{x}$ WAREHOUSING $28.00 \%$ $O H \& P$ (ON MARKUPS ONLY)

TOTAL COST CODE 70015 HBS 310308 (ESCALATION

$1.71 \%$ - CONTINGENCY
DOE_R08 - ESTIMATE DETAIL BY WBS / COSY CODE

(FIELD)

\footnotetext{
$20.00 \%)$
}

$\begin{array}{lcl}\text { PAGE } & 61 \\ \text { DATE } & 07 / 24 / 96 \quad 11: 28: 54\end{array}$

BY JPM SMF OKH LGH

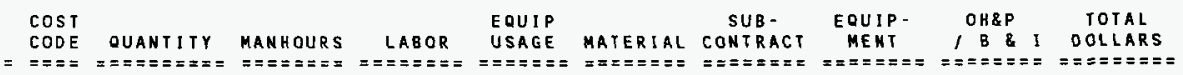

$\begin{array}{cccccccccc}700 \mathrm{~F} & 6 \mathrm{EA} & 24 & 1282 & 0 & 0 & 0 & 0 & 667 & 1949 \\ 700 \mathrm{~F} & 6 \text { EA } & 24 & 1282 & 0 & 0 & 0 & 0 & 667 & 1949\end{array}$

\subsection{5}

MECHANICAL

310308.1522324

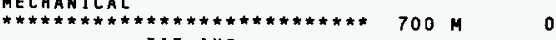

GLOVE BAE

1522326 MAKE 3 " SADDLEWELDS CS

310308.1522330 MAKE 2" SADDLEHELDS CS

310308.15223383 " DYE PEN HELD EXAM

$310308.15223402 "$ "DYE PEN WELD EXAM

310308.1522342 ALLOHANCE FOR LEAK DETECTOR

INSIDE IXM'S CURBED AREA

310308.1522344 ALIOW FOR REMOVAL \& PACKAGE

310308.1522501 PLACE IXM'S

SUBTOTAL MECHANICAL

SWP $100.00 \%$

GENERAL FOREMAN $7.00 \%$

CONSUMABLES $6.00 \%$

SALES TAX $8.00 \%$

WAREHOUSING $28.00 \%$

OH\&P (ON MARKUPS ONLY)

TOTAL COST CODE $700 \$ 5$

WBS 310308

\begin{tabular}{|c|c|c|c|c|c|}
\hline 700 & $\mathbf{M}$ & 0 & & 0 & 0 \\
\hline 700 & M & 2 & JOB & 16 & 854 \\
\hline $\begin{array}{l}700 \\
700 \\
700 \\
700 \\
700\end{array}$ & $\begin{array}{l}M \\
M \\
M \\
M \\
M\end{array}$ & $\begin{array}{l}4 \\
5 \\
4 \\
5 \\
1\end{array}$ & $\begin{array}{l}\text { EA } \\
\text { EA } \\
\text { EA } \\
\text { EA } \\
\text { LS }\end{array}$ & $\begin{array}{r}26 \\
26 \\
6 \\
6 \\
8\end{array}$ & $\begin{array}{r}1388 \\
1388 \\
320 \\
320 \\
427\end{array}$ \\
\hline 700 & $\mathbf{M}$ & 2 & JOB & 8 & 427 \\
\hline 700 & $\mathbf{M}$ & 3 & EA & 48 & 1720 \\
\hline & (MASK) & & & $\begin{array}{r}144 \\
144 \\
20\end{array}$ & $\begin{array}{r}6,844 \\
6844 \\
958\end{array}$ \\
\hline
\end{tabular}

...

0

33,213

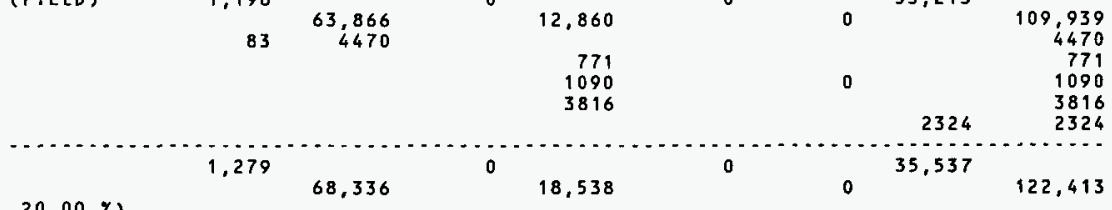

.

00000


WHC-SD-SNF-RPT-012, Rev. 0

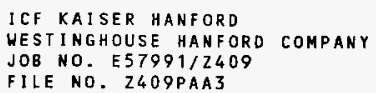

BY JPM SMF DKH LGH

TOTAL HBS 310308 TRANSFER BAY MODIFICAIIONS

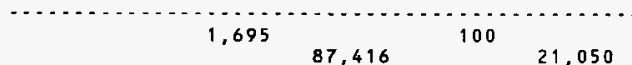

87,416

21,050

1,050
MATERIAL CONTRACT

EQUIP.

MENT

OH\&P

B \& I DOLLARS

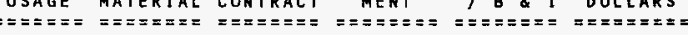

$\begin{array}{llll}21,050 & 0 & 45,459 & 154,026\end{array}$


WHC-SD-SNF-RPT-012, Rev. 0

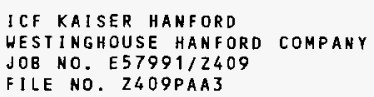

\section{ACCOUNT \\ NUMBER}

DESCR!PI ION

* * IEST - INTERACTIVE ESTIMATING * *

K-BASINS INTEGRATED HATER SYSTEM PREL I MIIMARY

DOE_ROB - ESTIMATE DETAIL BY WBS / COST CODE

\section{COST EQUIP}

CODE QUANTITY MANHOURS LABOR USAGE
PAGE 63

DATE $07 / 24 / 96 \quad 11: 28: 55$

BY JPM SMF DKH LGH

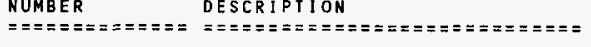

SUB-
MATERIAL CONTRACT

$\begin{array}{ll}\text { EQUIP- } & \text { OH\&P } \\ \text { MEHT TOTAL } & \quad \text { B \& I DOLLARS }\end{array}$

310309

DELID STATION

310309.15

MECHAN 1 CAL

310309.1522004 FIELD FAB

310309.1522008 PUMP P-KH-2 1506PM 75 PSI MIG DEVICE BY OTHERS

310309.1522012 MIG DIPE SCH 40 CSTL

310309.1522012 q" PIPE SCH 40 CSTL
310309.1522018 1" HOSE W/ FLG ENDS (LENGTH AS SUMED)

310309.1522020 11" ELB 90 SCRO MI 150\#

310309.1522025 1" FLANGE SCRD 150\# CS

310309.1522028 FAB HOSE PIPE SUPPORT (SST)

310309.1522032 HYDRO \& FLUSH

310309.9522130 INSTALLATION

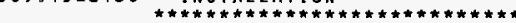

$310309.15221361 "$ HOSE INSIALLATION

310309.152214011 PIPE SPDOL

310309.1522144 PUMP P-KH-2 150 GPM

310309.1522148 1" FLG BOLTUP (INCLS BOLT $S E T$ \& GSKT)

310309.1522152 INSTALL 1" HOSE PIPE SUPPORT

310309.152215611 SST U-BOLT \& DRILL? HOLES FOR EACH U-BOLT THRU

310309.1522158 ALLOHANCE FOR MECHANICAL ATP ASSISTANCE

SUBTOTAL MECHANICAL

GENERAL FOREMAN $7.00 \%$

CONSUMABLES $6.00 \%$

SALES TAX $8.00 \%$

WAREHOUSING $28.00 \%$

OH\&P (ON MARKUPS ONLY)

TOTAL

COST CODE 70015

WBS 310309

$1.71 \%$ - CONTINGENCY $20.00 \%$ )

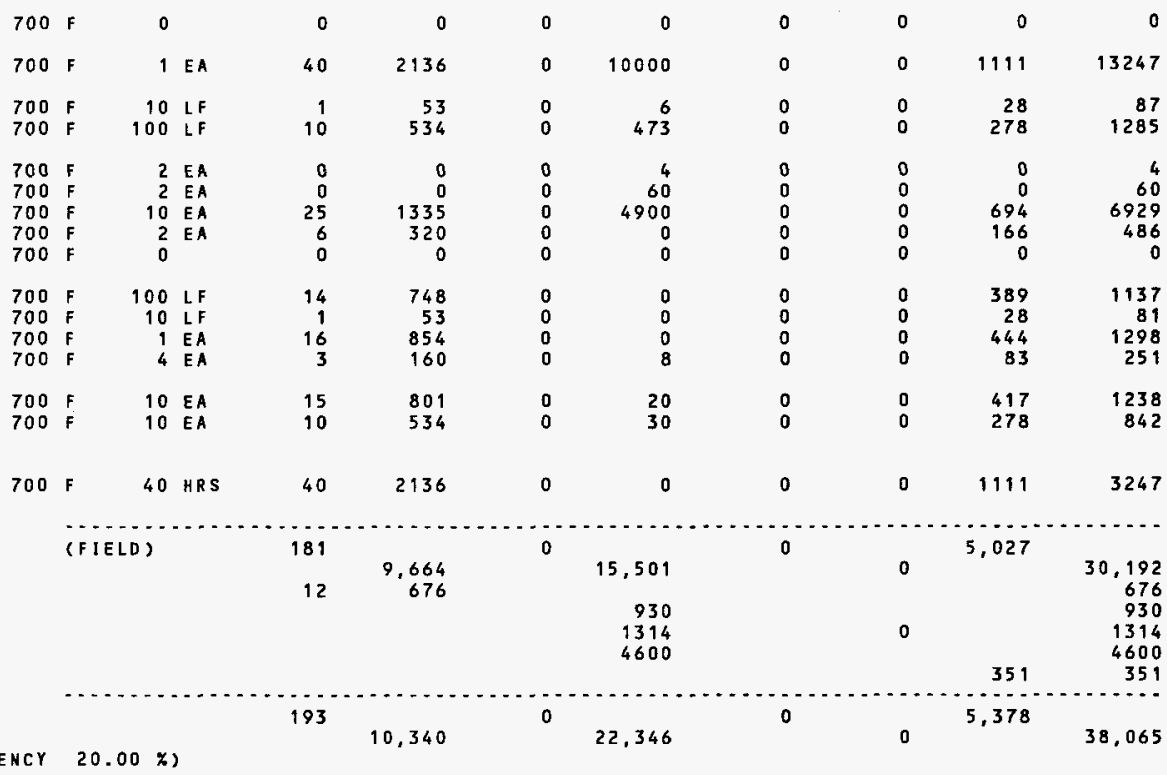


WHC-SD-SNF-RPT-012, Rev. 0

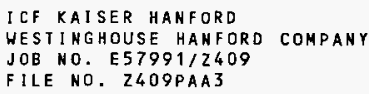

PAGE 65

DATE $07 / 24 / 96 \quad 11: 28: 55$

BY JPM SMF DKH LGH

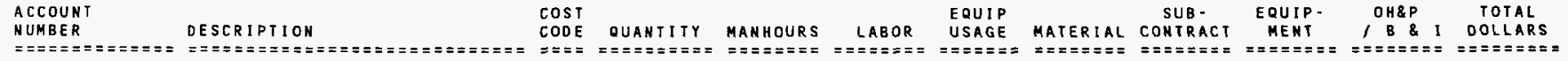

\section{1 \\ DOHN DRAFT TABLE \& DRY CANISTER SIA}

310311.15

310311.1581004 MECHANICAL

$* * * * * * * * * * * * * * * * * * * * * * * * * * *$
FIELD FAB CLEANING STATION

$700 \mathrm{~F}$

PUMP $P-K H=4$

310311.1581006420 GPM 60 PSI SUBMERSIBLE PUMP (LABOR TO HANDLE ONLY - MOUNTING DEIVCE BY OTHERS

$310311.15810084^{\prime \prime}$ 310311.1581010211 $310311.15810124^{\prime \prime}$ $S C H 40 \mathrm{C} / \mathrm{S}$ PIPE $310311.1581014 \quad 4 "$ HOSE H/FLG ENDS HOSE

$310311.15810164^{\prime \prime}$

C/S BW TEE

310311.95810184 "

$C / S B H$

FAB HOSE PIPE SUPPORT SST WITH ELOAT CHAIN

310311.1581022 FAB 4" PIPE SUPPORT $310311.15810242 "$ SST U-BOLTS

$310311.15810264 "$ SST U-BOLTS

310311.158102841 FLANGED GATE VALVE

310311.1581030411

$310311.15810324 "$

$15810324 "$ DYE PEN

10311.1581034 HYDRO \& FLUSH

SCH $40 \mathrm{C} / \mathrm{S}$ PIPE

$310311.15810402 "$ C/S WN FLANGE

310311.1581042 2" FIELDB/WELD

SUBTOTAL MECHANICAL

GENERAL FOREMAN $7.00 \%$

CONSUMABLES $6.00 \dot{x}$

SALES TAX $8.00 \%$

WAREHOUSING $28.00 \%$

OH\&P (ON MARKUPS ONLY)

TOTAL COST CODE 70015

WBS 310311

700

1 EA

40

2136

20000

1111

23247

\begin{tabular}{|c|c|c|c|}
\hline $\begin{array}{l}700 \\
700 \\
700 \\
700 \\
700 \\
700 \\
700\end{array}$ & $\begin{array}{l}F \\
F \\
F \\
F \\
F \\
F \\
F\end{array}$ & $\begin{array}{r}30 \\
25 \\
190 \\
2 \\
4 \\
6 \\
19\end{array}$ & $\begin{array}{l}\text { LF } \\
\text { LF } \\
\text { LF } \\
\text { EA } \\
\text { EA } \\
\text { EA } \\
\text { EA }\end{array}$ \\
\hline $\begin{array}{l}700 \\
700 \\
700 \\
700 \\
700 \\
700 \\
700 \\
700 \\
700 \\
700 \\
700 \\
700\end{array}$ & $\begin{array}{l}\mathbf{F} \\
\mathbf{F} \\
\mathbf{F} \\
\mathbf{F} \\
\mathbf{F} \\
\mathbf{F} \\
\mathbf{F} \\
\mathbf{F} \\
\mathbf{F} \\
\mathbf{F} \\
\mathbf{F} \\
\mathbf{F}\end{array}$ & $\begin{array}{r}3 \\
3 \\
19 \\
1 \\
20 \\
20 \\
4 \\
2 \\
10 \\
2 \\
2 \\
2\end{array}$ & $\begin{array}{l}E A \\
E A \\
E A \\
E A \\
E A \\
E A \\
E A \\
E A \\
L F \\
E A \\
E A \\
E A\end{array}$ \\
\hline
\end{tabular}

$\begin{array}{rr}11 & 587 \\ 5 & 267 \\ 67 & 3578 \\ 1 & 53 \\ 2 & 107 \\ 4 & 214 \\ 48 & 2563\end{array}$

$\begin{array}{rr}0 & 105 \\ 0 & 136 \\ 0 & 3040 \\ 0 & 100 \\ 0 & 96 \\ 0 & 240 \\ 0 & 490\end{array}$

$\begin{array}{rrrr}0 & 0 & 305 & 997 \\ 0 & 0 & 139 & 542 \\ 0 & 0 & 1861 & 8479 \\ 0 & 0 & 28 & 181 \\ 0 & 0 & 56 & 259 \\ 0 & 0 & 111 & 565 \\ 0 & 0 & 1333 & 4386 \\ 0 & 0 & 83 & 363 \\ 0 & 0 & 83 & 258 \\ 0 & 0 & 528 & 1866 \\ 0 & 0 & 28 & 930 \\ 0 & 0 & 3665 & 10714 \\ 0 & 0 & 999 & 2921 \\ 0 & 0 & 444 & 1298 \\ 0 & 0 & 56 & 179 \\ 0 & 0 & 56 & 189 \\ 0 & 0 & 28 & 141 \\ 0 & 0 & 222 & 649 \\ 0 & 0 & 56 & 163\end{array}$

(FIELD)

03

160
160

160
1015

53

7049

1922

854

107
107

53
427

107

$0 \quad 120$

120
15
323

323
849

...

11,192

163

$28 \quad 21,519$

25,616

0

58,327

1506

1536

2172

2172

0

783

783

43

23,025

36,928

0

11,975

71.928 
WHC-SD-SNF-RPT-012, Rev. 0

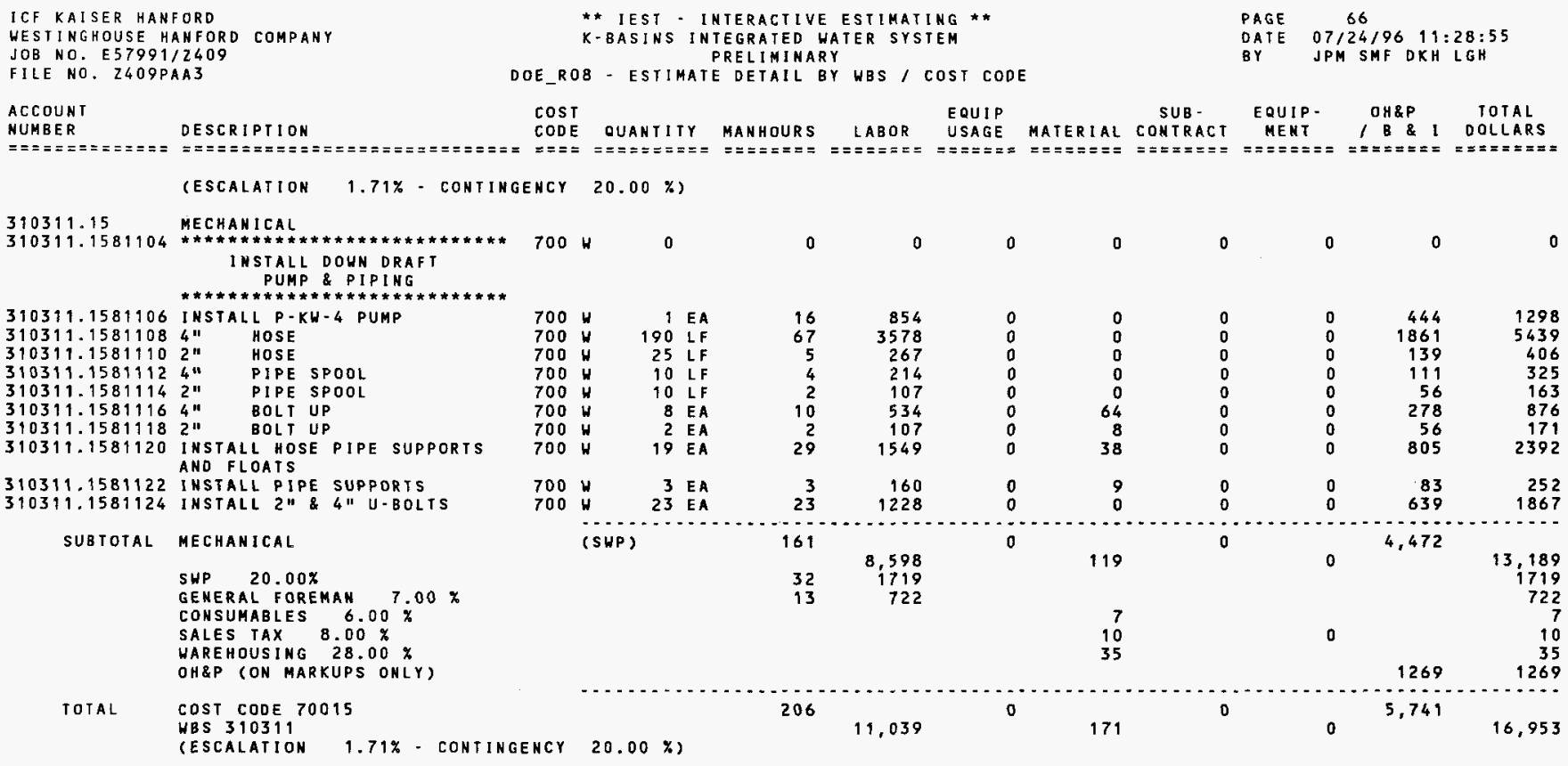

TOTAL WBS 310311 DOWH DRAFT TABLE \& DRY CANISTER STA

637

34,065

0

37,099

0

17,717

88,881 
WHC-SD-SNF-RPT-012, Rev. 0

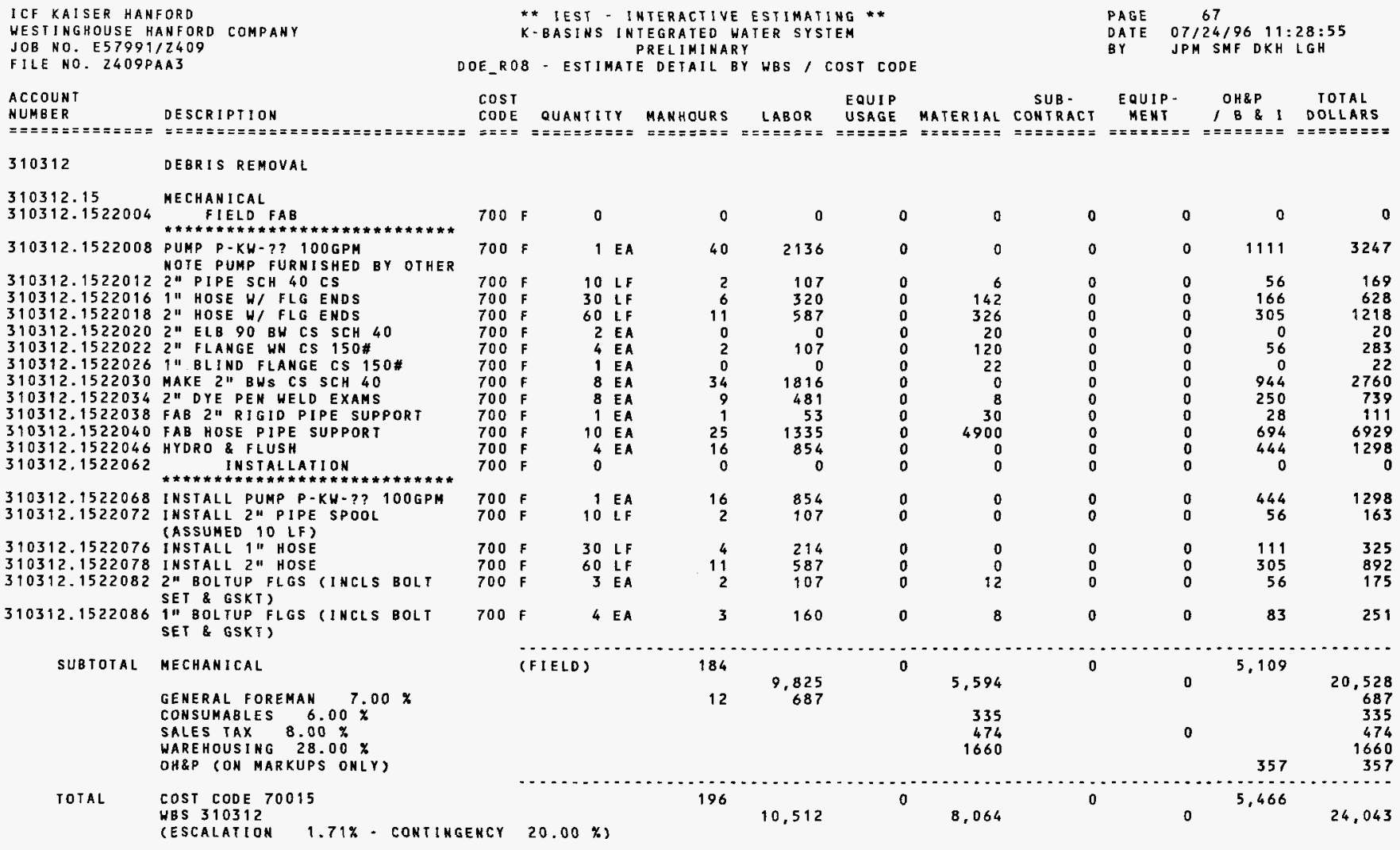


WHC-SD-SNF-RPT-012, Rev. 0

ICF KAISER HANFORD

WESTINGHOUSE HAHFORD COMPANY

JOB NO. E57991/Z409

FILE NO. 2409 PAA3

ACCOUNT

NUMBER

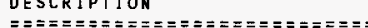

TOTAL HBS 310312 DEBRIS REMOVAL
* * IEST - INTERACTIVE ESTIMATING **

K-BASINS INTEGRATED WATER SYSTEM PRELIMINARY

DOE_R08 - ESTIMATE DETAIL BY WBS / COST CODE

COST

CODE QUANTITY MANHOURS LABOR USAGE

$\begin{array}{lcccc}\text { SUB - } & \text { EQUIP- } & \text { OH\&P } & \text { TOTAL } \\ \text { MATERIAL CONTRACT MENT } & \text { B \& DOLLARS }\end{array}$

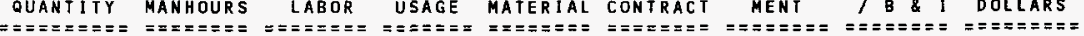

PAGE 68 DATE $07 / 26 / 96,11: 28: 55$

DATE $07 / 24 / 96$ TI:28:55
196

10,512
0

8,064
0
5,466 
ICF KAISER HANFORD

WESTINGHOUSE HANFORD COMPANY JOB NO. E $57991 / 2409$

FILE NO. Z409PAA3

ACCOUNT

A

DESCRIPIION

SW LOAD OUT PIT

310313

MECHANICAL

310313.15
310313.1522004

FIELD FA

310313.152200611 PIPE SCH 40 CS

310313.1522012 4" PIPE SCH 40 CS

(ASSUHED LENGTH)

310313.1522016 1" THREADOLET CS

310313.152201941 "THREADOLET CS

310313.1522021 1" FLANGE SCRD CS 150 \#

310313.1522025 4" FLANGE WN CS 150\#

310313.1522030 1" ELB 90 SCR 150\#

310313.1522034 I" ELB 90 SCR 150\# (SHOH

LABOR WITH INSTALLATION FOR THESE 7 ELBOHS)

$310313.15220384 " \mathrm{ELB} 90 \mathrm{BW}$ SCH $40 \mathrm{CS}$

$310313.15220444 \mathrm{ll} B W$ SCH 40 CS

310313.1522046 4" DYE PEN HELD EXAM

310313.1522054 FAB 4 " PIPE SUPPORT

310313.1522064 FAB 1" PIPE SUPPORT

310313.1522084 1" GATE VALVE SCRD CS

310313.1522088 4" GATE VALVE FLGD CS

310313.1522208 INSTALLATION

310313.1522218 1" PIPE SCH $40 \mathrm{CS}$

310313.1522228 4" PIPE SCH $40 \mathrm{CS}$

310313.152224011 FLG BOLTUPS (MRTL INCLS

310313.1522244 4" FLG BOLTUPS (MRTL IHCLS BOLT SET \& GSKI)

310313.1522248411 BW CS SCH 40

310313.1522252 INSTALL 1" ELB 90 SCRD 150\#

310313.1522259 INSTALL 4" PIPE SUPPORT

310313.1522268 INSTALL 11 PIPE SUPPOR

310313.1522308 HYDRO \& FLUSH

$310313.15224081 "$ THREADOLET CS

3103131522416 (PURCHSED IN 'FAB')

$310313.15224164 "$ THREADOLET CS
$310313.15224531 "$ SADDLEHELD CS 310313.1522453 1" SADDLEWELD CS
310313.1522456 4" SADDLEWELD CS

SUBTOTAL MECHANICAL
* * IEST - INTERACTIVE ESTIMATING * *

K-BASINS INTEGRATED WATER SYSTEM

PRELIMINARY

DOE_ROS - ESTIMATE DETAIL BY WBS / COST CODE
PAGE 69

DATE $07 / 24 / 96 \quad 11: 28: 55$

COST EQUIP SUB- EQUIP- OH\&P TOTAL

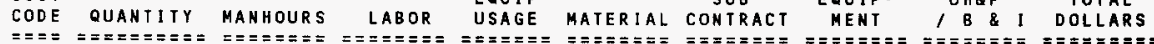

$\begin{array}{rrrr}700 & F & 0 \\ 700 & F & 70 & \text { LF } \\ 700 & F & 70 & \text { LF } \\ 700 & F & 1 & \text { EA } \\ 700 & F & 1 & \text { EA } \\ 700 & F & 3 & \text { EA } \\ 700 & F & 3 & \text { EA } \\ 700 & F & 7 & \text { EA } \\ 700 & F & 7 & \text { EA }\end{array}$

$\begin{array}{rr}0 & \\ 10 & 534 \\ 25 & 1335 \\ 0 & \\ 0 & \\ 1 & 5 \\ 2 & 107 \\ 4 & 21 \\ 0 & \end{array}$

$\begin{array}{rr}0 & 0 \\ 534 & 0 \\ 1335 & 0 \\ 0 & 0 \\ 0 & 0 \\ 53 & 0 \\ 107 & 0 \\ 214 & 0 \\ 0 & 0\end{array}$

0
39
245
4
5
66
120
14
14

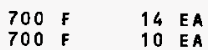

$700 \mathrm{~F}$

$700 \mathrm{~F}$

$700 \mathrm{~F}$

$700 \mathrm{~F}$

$700 \mathrm{~F}$

$700 \mathrm{~F}$

10 EA

7 EA

7 EA

1 EA

1 EA

o

$\begin{array}{rr}6 & 320 \\ 66 & 352 \\ 18 & 96 \\ 8 & 427 \\ 7 & 374 \\ 0 & \\ 1 & 53 \\ 0 & 0\end{array}$

$700 \mathrm{~F} \quad 70 \mathrm{LF}$

$700 \mathrm{~F} \quad 70 \mathrm{LF}$

$10 \quad 534$

00

3 EA

10
25
2

$700 \mathrm{~F} \quad 3$ EA

$700 \mathrm{~F} \quad B$ EA

$700 \mathrm{~F}$

$700 \mathrm{~F}$

$700 \mathrm{~F}$

$700 \mathrm{~F}$

$700 \mathrm{~F}$

7 EA

7 EA

7 EA

2 EA

107

$4 \quad 214$

$\begin{array}{lll}700 & F & 1 \\ 700 & F A\end{array}$

$\begin{array}{ll}700 & F \\ 700 & F\end{array}$

1 EA

(FIELD)

...

$53 \quad 2830$

$\begin{array}{ll}4 & 214 \\ 7 & 374\end{array}$

$5 \quad 267$

$12 \quad 641$

$320 \quad 350$

24

427

74

0
53

10
10
280

280

105
30

30
849

53

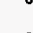

$$
\begin{aligned}
& 0 \\
& 0 \\
& 6
\end{aligned}
$$

24

$\begin{array}{rr}1 & 53 \\ 5 & 267\end{array}$

$\begin{array}{ll}5 & 267 \\ 8 & 427\end{array}$

285

15,218

18

0

2,207

$\begin{array}{rr}0 & 0 \\ 278 & 851 \\ 694 & 2274 \\ 0 & 4 \\ 0 & 5 \\ 28 & 147 \\ 56 & 283 \\ 111 & 339 \\ 0 & 14\end{array}$

274

166
1832

1832
500

222

194

0
28
0

836
5366

1471

1471
929

673

30
930

0

278

694
56

812

2029

169

349

111

4302

1472
111

111

194

139
333

325

58

420

974
81

$c-85$ 
WHC-SD-SNF-RPT-012, Rev. 0

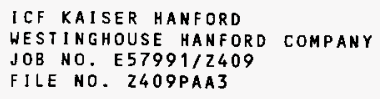

IOTAL

\section{COST CODE 70015 \\ WBS 310313} (ESCALATION

$1.71 \%-$ CONTINGEHCY
* * IEST - INTERACTIVE ESTIMATING * *

K-BASINS INTEGRATED WATER SYSIEM PRELIMINARY

DOE_RO8 - ESTIMATE DETAIL BY HBS / COST CODE

\section{$\cos \mathrm{T}$}

QODE QUANTITY MANHOURS LABOR USAGE

SUB -

SUB-

PAGE 70

DATE $07 / 24 / 96 \quad 11: 28: 55$ BY JPM SMF DKH LGH

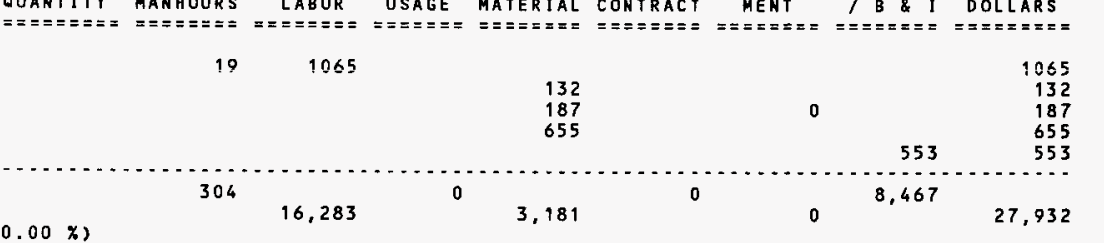

TOTAL WBS 310313 SH LOAO OUT PIT

304

16,283

3,981

0

8,467

27,932 
WHC-SD-SNF-RPT-012, Rev. 0

\begin{tabular}{|c|c|c|c|c|c|c|c|c|c|c|c|}
\hline $\begin{array}{l}\text { 1CF KAISER HANF } \\
\text { WESTINGHOUSE H } \\
\text { JOBNO. EST991 } \\
\text { FILENO. Z4O9P }\end{array}$ & $\begin{array}{l}\text { FORD } \\
\text { ANFORD COMPANY } \\
\text { IZOO } \\
\text { AA3 }\end{array}$ & $O E_{-} R O$ & $\begin{array}{l}\text { ** IESI - IN } \\
\text { K-BASINS INT } \\
08-\text { ESIIMATE }\end{array}$ & $\begin{array}{l}\text { NTERACTIVE } \\
\text { TEGRATED H } \\
\text { PRELIMINAR } \\
\text { E DETAIL B }\end{array}$ & $\begin{array}{l}\text { E ESTIMAT } \\
\text { HATER SYS } \\
\text { QY } \\
\text { GY HBS I }\end{array}$ & $\begin{array}{l}\text { ING ** } \\
\text { TEM } \\
\operatorname{COST} \cos \end{array}$ & & & $\begin{array}{l}\text { PAGE } \\
\text { DAIE } \\
\text { BY }\end{array}$ & $\begin{array}{l}79 \\
07 / 24 / 96 \quad 11: \\
\text { JPM SMF DKH }\end{array}$ & $\begin{array}{l}28: 56 \\
\text { LGH }\end{array}$ \\
\hline $\begin{array}{l}\text { ACCOUNT } \\
\text { NUMBER } \\
===-=======0\end{array}$ & 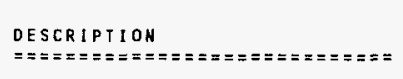 & $\begin{array}{l}\operatorname{COST} \\
\operatorname{CODE} \\
====\end{array}$ & $====x======$ & $\begin{array}{l}\text { MAN HOUR S } \\
=======\end{array}$ & $\begin{aligned} & \text { LABOR } \\
&===== \pm=\end{aligned}$ & $\begin{array}{l}\text { EQUIP } \\
\text { USAGE } \\
=======\end{array}$ & $\begin{array}{l}\text { MATERIAL } \\
=======\end{array}$ & $\begin{array}{l}\text { SUB- } \\
\text { CONTRACT } \\
========\end{array}$ & $\begin{array}{l}\text { EQUIP - } \\
\text { MENT } \\
=======\end{array}$ & $===\begin{array}{r}O H \& P \\
B \& \&\end{array}$ & $\begin{aligned} & \text { TOTAL } \\
& \text { DOLLARS } \\
&=======\end{aligned}$ \\
\hline 310314 & DEMINERALIZED WATER & & & & & & & & & & \\
\hline $\begin{array}{l}310314.15 \\
310314.1522004\end{array}$ & 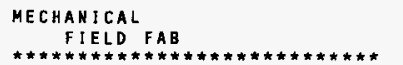 & 700 & o & 0 & 0 & 0 & 0 & 0 & & 0 & 0 \\
\hline $\begin{array}{l}310314.1522005 \\
310314.1522008 \\
310314.1522012 \\
310314.1522016 \\
310314.1522018 \\
310314.1522020 \\
310314.1522024 \\
310314.1522028 \\
310314.1522032\end{array}$ & 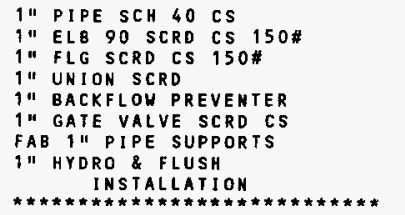 & $\begin{array}{l}700 \\
700 \\
700 \\
700 \\
700 \\
700 \\
700 \\
700 \\
700\end{array}$ & $\begin{array}{rl}20 & \text { LF } \\
4 & E A \\
1 & E A \\
3 & E A \\
1 & E A \\
1 & E A \\
3 & E A \\
1 & E A \\
0 & \end{array}$ & $\begin{array}{l}3 \\
0 \\
0 \\
0 \\
1 \\
1 \\
3 \\
4 \\
0\end{array}$ & $\begin{array}{r}160 \\
0 \\
0 \\
0 \\
53 \\
53 \\
160 \\
214 \\
0\end{array}$ & $\begin{array}{l}0 \\
0 \\
0 \\
0 \\
0 \\
0 \\
0 \\
0 \\
0\end{array}$ & $\begin{array}{r}11 \\
6 \\
30 \\
11 \\
400 \\
30 \\
45 \\
0 \\
0\end{array}$ & $\begin{array}{l}0 \\
0 \\
0 \\
0 \\
0 \\
0 \\
0 \\
0 \\
0\end{array}$ & & $\begin{array}{r}83 \\
0 \\
0 \\
0 \\
28 \\
28 \\
83 \\
119 \\
0\end{array}$ & $\begin{array}{r}254 \\
6 \\
30 \\
11 \\
481 \\
111 \\
288 \\
325 \\
0\end{array}$ \\
\hline $\begin{array}{l}310314.1522036 \\
310316.1522038 \\
310314.1522040 \\
310314.1522044\end{array}$ & $\begin{array}{l}\text { 1" PIPE SPOOL } \\
\text { 1" PIPE SUPPORT INSTL } \\
\text { INSTALL BACKFLOH PREVENTOR } \\
\text { MAKE 1" CONNECTION TO EXISTI } \\
\text { NG 1" PIPE }\end{array}$ & $\begin{array}{l}700 \\
700 \\
700 \\
700\end{array}$ & $\begin{array}{rl}20 & \mathbf{L F} \\
3 & \mathrm{EA} \\
1 & \mathrm{EA} \\
1 & \mathrm{EA}\end{array}$ & $\begin{array}{l}3 \\
2 \\
3 \\
4\end{array}$ & $\begin{array}{l}160 \\
107 \\
160 \\
214\end{array}$ & $\begin{array}{l}0 \\
0 \\
0 \\
0\end{array}$ & $\begin{array}{l}0 \\
9 \\
0 \\
0\end{array}$ & $\begin{array}{l}0 \\
0 \\
0 \\
0\end{array}$ & & $\begin{array}{r}83 \\
56 \\
83 \\
111\end{array}$ & $\begin{array}{l}243 \\
172 \\
243 \\
325\end{array}$ \\
\hline 310314.1522048 & $\begin{array}{l}\text { ALLOWANCE FOR UPGRADE EXIST } \\
\text { DEMINERALIZER }\end{array}$ & 700 & $\begin{array}{l}1 \mathrm{EA} \\
\ldots \ldots \ldots\end{array}$ & 16 & 854 & 0 & 5000 & 0 & & 444 & 6298 \\
\hline SUBTOTAL & $\begin{array}{l}\text { MECHANICAL } \\
\text { GENERAL FOREMAN } 7.00 \% \\
\text { CONSUMABLES } 6.00 \% \\
\text { SALES TAX } 8.00 \% \\
\text { WAREHOUSING } 28.00 \% \\
\text { OH\&P (ON MARKUPS ONLY) }\end{array}$ & & (FIELD) & $\begin{array}{r}40 \\
2\end{array}$ & $\begin{array}{r}2,135 \\
149\end{array}$ & 0 & $\begin{array}{r}5,542 \\
332 \\
469 \\
1644\end{array}$ & 0 & & 1,110 & $\begin{array}{r}8,787 \\
149 \\
332 \\
469 \\
1644 \\
77\end{array}$ \\
\hline IOTAL & $\begin{array}{l}\text { COST CODE } 70015 \\
\text { HBS } 310314 \\
\text { (ESCALATION } 1.71 \% \cdot \text { CONTINGE }\end{array}$ & & $20.00 \%)$ & 42 & 2,284 & 0 & 7,989 & 0 & & 1,187 & 11,461 \\
\hline
\end{tabular}

TOTAL WBS 310314 DEMINERALIZED WATER

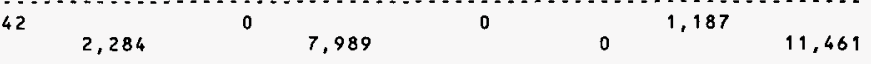


WHC-SD-SNF-RPT-012, Rev. 0

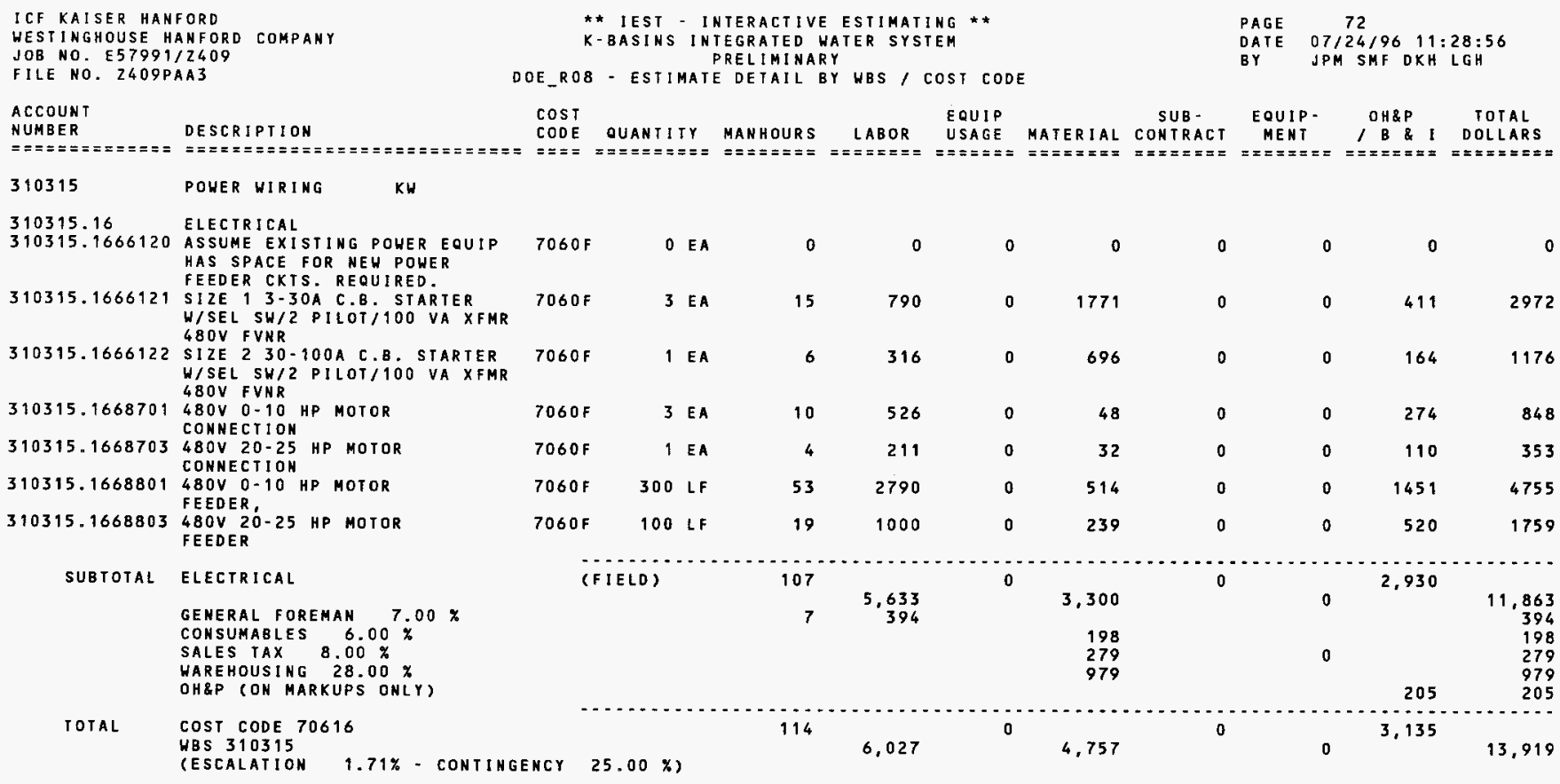


WHC-SD-SNF-RPT-012, Rev, 0

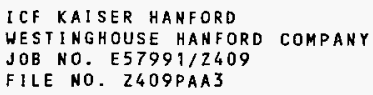

\section{ACCOUNT}

NUMBER

DESCRIPIION

$=== \pm==$

310316

I NSTRUMENTATIOH

310316.15 MECHANICAL

310316.1593005 1/4" TUBING COPPER

310316.159300714 EA $\times 2$ LF

310316.1593019 NOTE: TAKEOFF IS PER DHG, ALL INSTRUMENTS SHOWN ARE TAKEN AS NEU (UNLESS SPECIF1 CALLY LABELED OTHERHISE)

310316.1593025 PI FI (SEE ELECTRICAL FOR 310316.1593027 ACTUAL INSTRUMENT PURCHASE EIC

310316.1593029 /4" THREADDLETS, CS

$310316.15930351 / 411 \times 2$ PIPE NIPPLE A106 CSTL

$310316.15930361 / 4 "$ TEE SCRD CS

$310316.15930381 / 4 "$ BALL VALVE, BRASS

\section{SUBTOTAL MECHANICAL}

GENERAL FOREMAN $7.00 \%$

CONSUMABLES $6.00 \%$

SALES TAX $8.00 \%$

WAREHOUSING $28.00 \%$

OH\&P (ON MARKUPS ONLY)

TOIAL COST COOE 70015

HBS 310316

(ESCALATION $1.71 \%$ - CONTINGENCY $20.00 \%$ )
* * IEST - INTERACTIVE ESTIMAIING * *

K-BASINS INTEGRATED HATER SYSTEM PRELIMINARY

DOE_ROB - ESTIMATE DETAIL BY WBS / COST CODE
PAGE 73

DATE $07 / 24 / 96 \quad 11: 28: 56$

BY JPM SMF DKH LGH

COST EQUIP SUB- EQUIP- OH\&P TOTAL

CODE OUANTITY MANHOURS LABOR USAGE MATERIAL CONTRACT MENT / B \& I DOLLARS

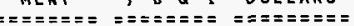

$\begin{array}{rrrrrrrrrr}700 \mathrm{~F} & 8 \text { LF } & 2 & 107 & 0 & 4 & 0 & 0 & 56 & 167 \\ 700 \mathrm{~F} & 8 \text { EA } & 3 & 160 & 0 & 32 & 0 & 0 & 83 & 275 \\ 700 \mathrm{~F} & \text { O } & 0 & 0 & 0 & 0 & 0 & 0 & 0 & 0\end{array}$

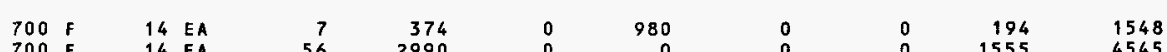

$\begin{array}{lllllllllll}700 \mathrm{~F} & 7 & \mathrm{EA} & 2 & 107 & 0 & 35 & 0 & 0 & 56 & 198\end{array}$

$\begin{array}{llllllllll}700 \mathrm{~F} & 33 \mathrm{EA} & 2 & 107 & 0 & 165 & 0 & 0 & 56 & 328\end{array}$

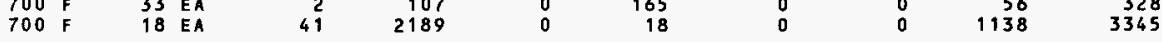

$\begin{array}{llllllllll}700 \mathrm{~F} & 33 \mathrm{EA} & 5 & 267 & 0 & 10 & 0 & 0 & 139\end{array}$

$\begin{array}{lllllllllll}700 \mathrm{~F} & 3 \mathrm{EA} & 2 & 107 & 0 & 2 & 0 & 0 & 56 & 165\end{array}$

$\begin{array}{rrrrrrrrrr}700 \mathrm{~F} & 3 \mathrm{EA} & 2 & 107 & 0 & 2 & 0 & 0 & 56 \\ 700 \mathrm{~F} & 36 \mathrm{EA} & 17 & 908 & 0 & 1800 & 0 & 0 & 472 & 3180\end{array}$

(FIELO)

$\begin{array}{rrrrr}9 & 7.316 & 3,046 & 0 & 14,167 \\ 512 & 312\end{array}$

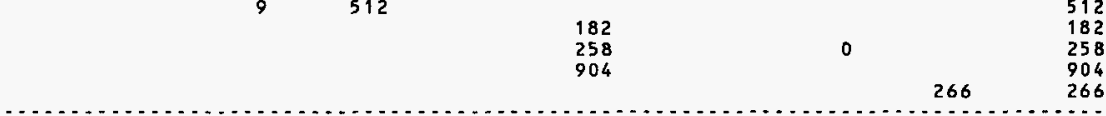

146

4,39

$0 \quad 4,079$

16,290 MECHAN I CAL

310316.15

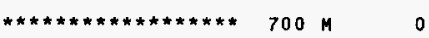
TIE INS

0

22

o

0

0

CSTL TO EXISITING PIPE 
WHC-SD-SNF-RPT-012, Rev. 0

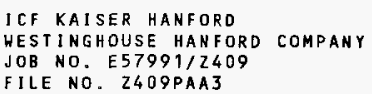

* * IEST - INTERACTIVE ESTIMATING * *

K-BASINS INIEGRATED WATER SYSTEM PREL I MINARY
PAGE 74

DATE $07 / 24 / 96 \quad 11: 28: 56$

BY JPM SMF DKH LGH

\begin{tabular}{|c|c|}
\hline $\begin{array}{l}\text { ACCOUNT } \\
\text { NUMBER } \\
============\pi\end{array}$ & 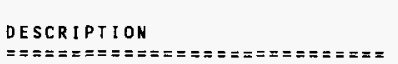 \\
\hline
\end{tabular}

OE RO8 - ESTIMATE DETAIL BY HBS / COST CODE

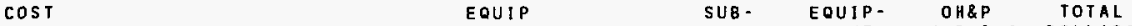

CODE OUANTITY MANHOURS SUB- EQUIP- OH\&P CODE QUANTITY MANHOURS LABOR USAGE MATERIAL CONTRACT MENT / B \& I DOLLARS

SUBTOTAL MECHANICAL

SWP $100.00 \%$

GENERAL FOREMAN $7.00 \%$

CONSUMABLES $6.00 \%$

SALES TAX $8.00 \%$

WAREHOUSING $28.00 \%$

OH\&P (ON MARKUPS ONLY)

TOTAL COST CODE 70015

HBS 310316

(ESCALATIOH $1.71 \%$ - CONTINGENCY $30.00 \%$ )

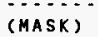

(MASK)

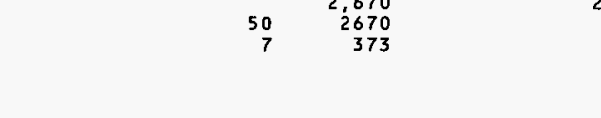

07

ELECTRICAL

310316.16

310316.1666001 INSTRUMENTATION TOTAL DOLLAR $7065 \mathrm{~F}$ INCLUDES INSTRUMENTANT AND ALLOWANCE FOR CND/WIRE/ TUBING THAT MAY BE REQUIRED

310316.1684600 EXISTING PLC KW ADD MISC

310316.1684604 EXISTING PLC KW ADD AI MODULES

310316.1684606 EXISIING PLC KW ADD DO MODULES

310316.1684608 EXISIING PLC KW

310316.1684608 EXISIING PLC KW

310316.1684612 ADD IC MODULES

HORK STATION

310316.1684614 NEH PLOTER

310316.1684616 LAN KH TO KE

AL LOW

310316.1684620 EXISTING MMI KH INSTALL IN ROOM 5

310316.1684622 EXISTING PLC KH

INSTALL IN ROOM 5

310316.1684650 CONNECT SIGNALS DI

310316.1684651 CONNECI SIGNALS AI

310316.1684652 CONNECT SIGNALS TC
$7065 \mathrm{~F} \quad 0$

$7065 F$

$7065 \mathrm{~F}$

$7065 \mathrm{~F}$

$7065 \mathrm{~F}$

$7065 \%$

$7065 \mathrm{~F}$

$7065 F$

$7065 \mathrm{~F}$

$7065 \mathrm{~F}$

$7065 F$

$7065 \mathrm{~F}$

$7065 F$

$7065 \mathrm{~F}$
$107 \quad 5,713$
1 EA

8 EA

$1 \mathrm{EA}$

1 EA

1 EA

1 EA

1 EA

$1 \mathrm{EA}$

1 EA

1 EA

7 EA

52 EA
3 EA
0

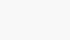

0

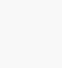

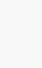

0

$\begin{array}{rrr}4 & 211 & 0 \\ 15 & 790 & 0 \\ 2 & 105 & 0 \\ 2 & 105 & 0 \\ 2 & 105 & 0 \\ 24 & 1263 & 0 \\ 7 & 368 & 0 \\ 91 & 4790 & 0 \\ 22 & 1158 & 0 \\ 64 & 3369 & 0 \\ & & \\ 3 & 158 & 0 \\ 25 & 1316 & 0 \\ 1 & 53 & 0\end{array}$

1040
1560
176
273
273
6170
3372
5909
400
430
2
17
1


WHC-SD-SNF-RPT-012, Rev. 0

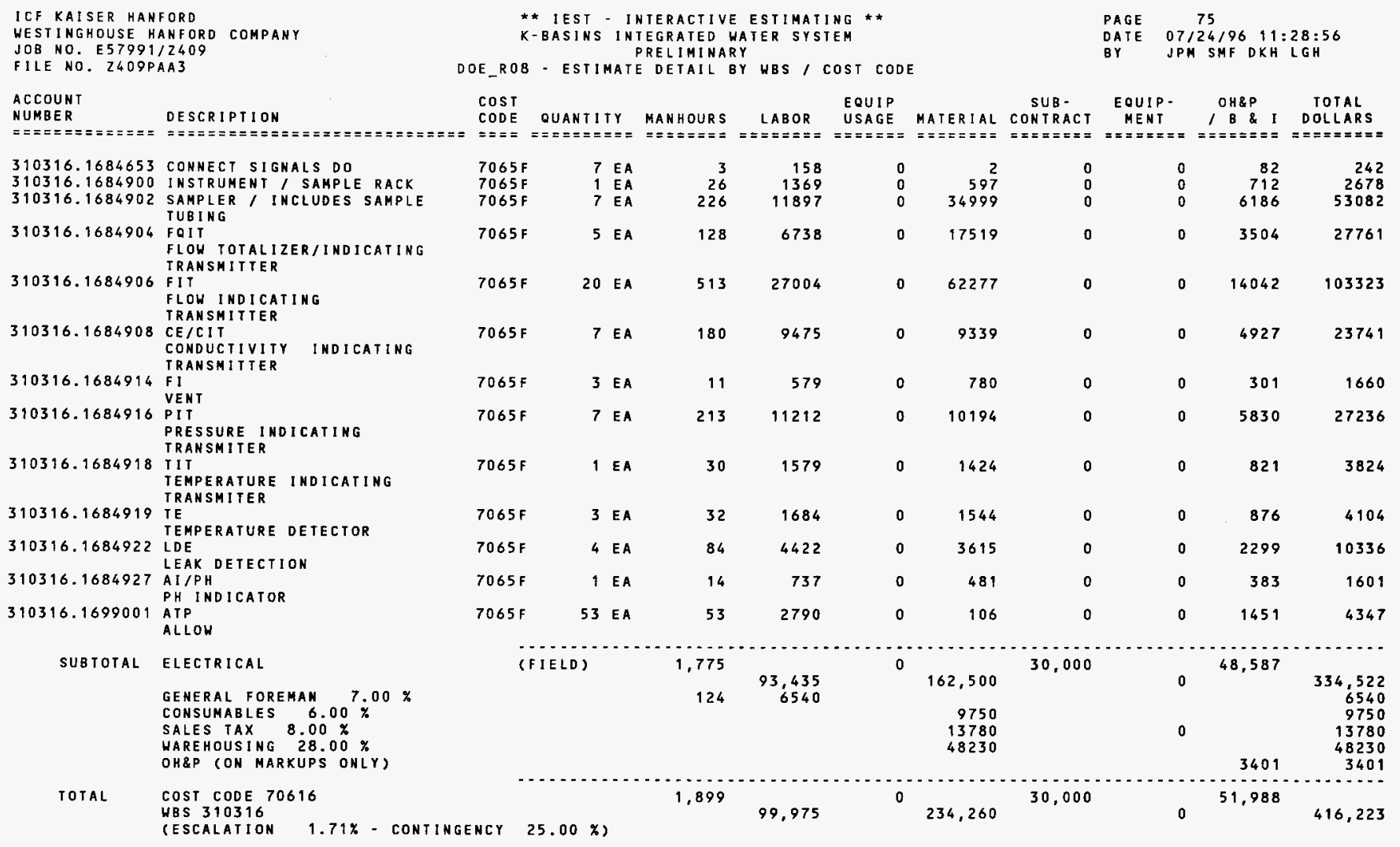


WHC-SD-SNF-RPT-012, Rev. 0
ICF KAISER HANFORD
WEST INGHOUSE HANFORD COMPANY
JOB NO. E57991/Z409
* * IEST - INTERACTIVE ESTIMATING *
K-BASIHS INTEGRATED WATER SYSTEM
PRELIMINARY

DOE ROB - ESTIMATE DETAIL BY WBS / COST CODE

\author{
COST OUATTITY MANHOURS LABOR UUIP
}

CODE QUANTITY MAHHOURS LABOR USAGE
ACCOUNT
DESCRIPTION
NUMBER

USAGE MATERIAL CONTRACT

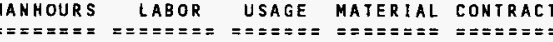

PAGE

(7) $24 / 96 \quad 11: 28: 56$

BY JPM SMF DKH LGH

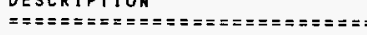

$==$

TOTAL WBS 310316 INSTRUMENTATION

KH
30,000

EQUIP -

OH\&P, TOTAL
MENT -

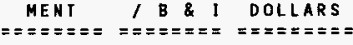

\begin{abstract}
2,152
\end{abstract}
113,517 o

238,682 
WHC-SD-SNF-RPT-012, Rev. 0

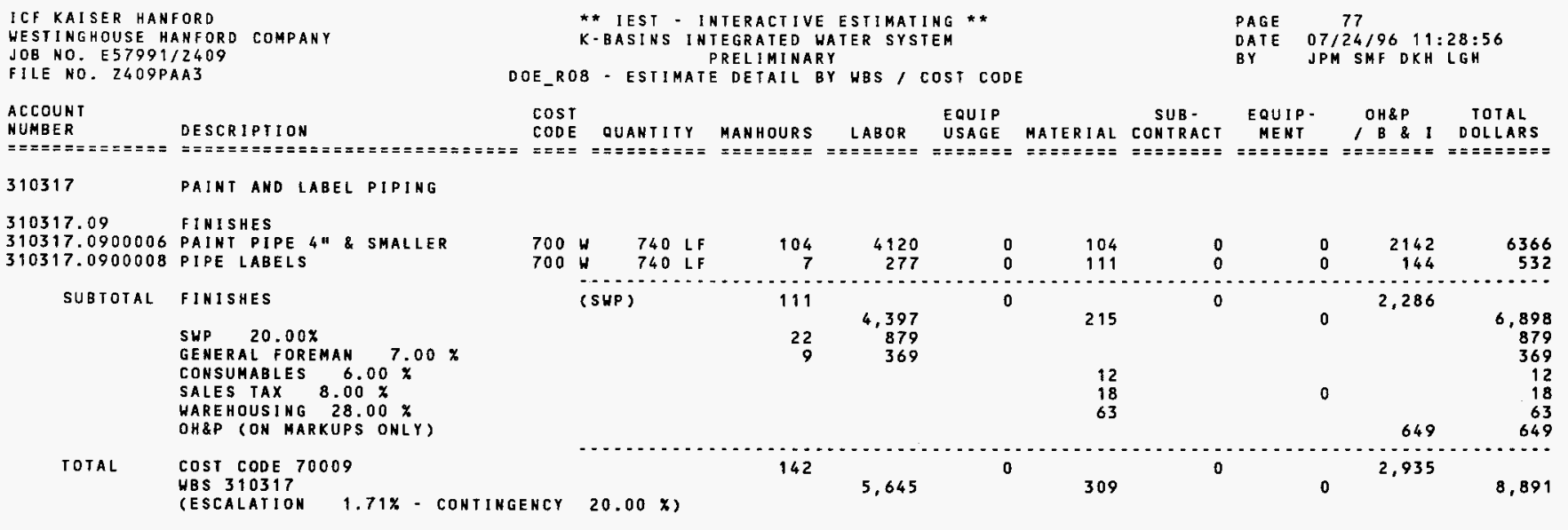

TOTAL HBS 310317 PAINT AND LABEL PIPING

162

5,645

o

309

0

2,935

8,891 
WHC-SD-SNF-RPT-012, Rev. 0

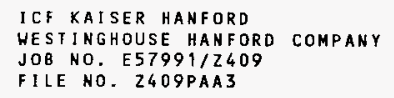

\section{ACCOUNT}

NUMBER

310380

DE SCR I P T I ON

BURNOUT

310380.20

BURNOUT

310380.2000002 BURNOUT

RAN MASK HOURS 292 X 1.85

$1.00=540$ BURNOUT HOURS

$1.00=540$ BURNOUT HOURS

SUBTOTAL BURNOUT

TOTAL

COST COOE 70020

HBS 310380

(ESCALATION

1. 71X - CONTINGENCY
* * IEST - INTERACTIVE ESTIMATING * *

K-BASINS INTEGRATED WATER SYSTEM

PRELIMINARY

DOE_R08 - ESTIMATE DETAIL BY WBS/ COSI CODE
PAGE 78

DATE $07 / 24 / 96 \quad 11: 28: 56$

BY JPM SMF DKH LGH

SUB - EQUIP- OH\&P TOTAL

CODE QUANTITY MANHOURS LABOR USAGE MATERIAL CONTRACT MENT I B \& I DOLLARS

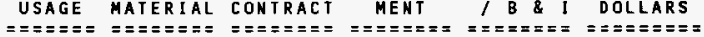

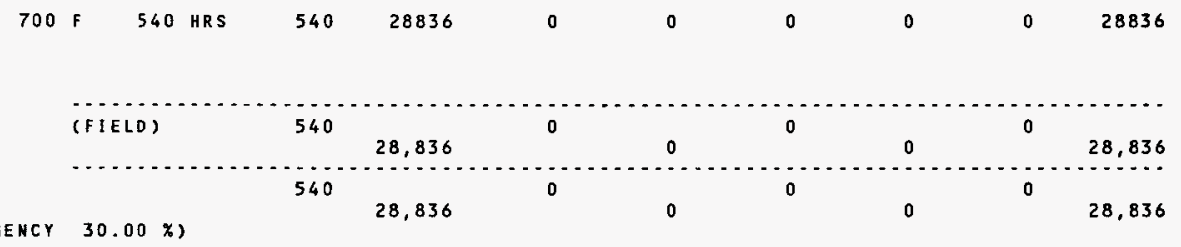

TOTAL WBS 310380 BURHOUT

540

0

0

0

28,836

0

0

28,836 
WHC-SD-SNF-RPT-012, Rev. O

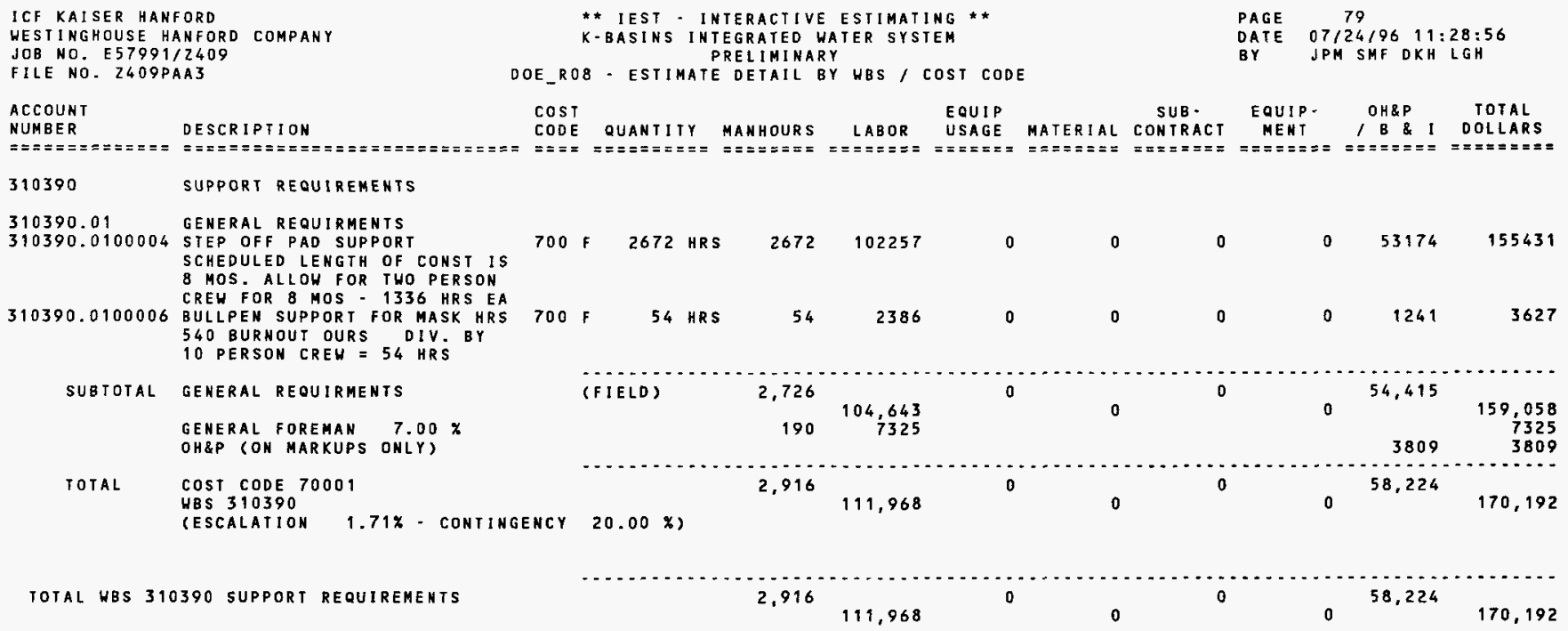


WHC-SD-SNF-RPT-012, Rev. 0

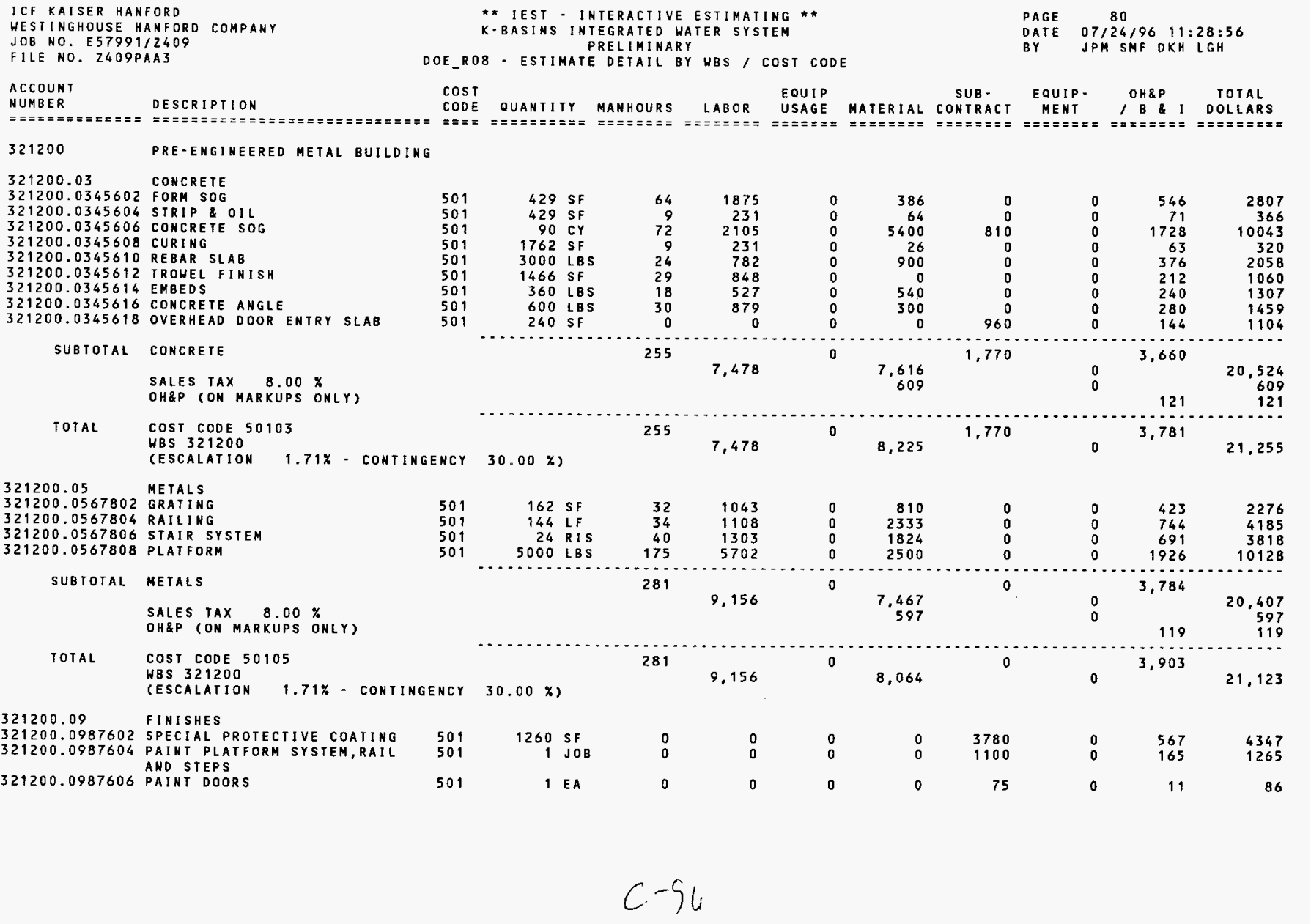


WHC-SD-SNF-RPT-012, Rev. 0

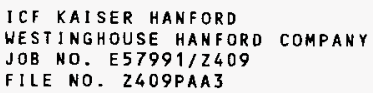

* * IEST - INTERACTIVE ESTIMATING * *

K-BASINS INTEGRATED HATER SYSTEM PRELIMINARY

DOE R08 - ESTIMATE DETAIL BY HBS / COST CODE
PAGE 81

DATE $07 / 24 / 96 \quad 11: 28: 57$

BY JPM SMF DKH LGH

COST EQUIP SUB - EQUIP- OH\&P TOTAL CODE QUANTITY MANHOURS LABOR USAGE MATERIAL CONTRACT MENT / B \& I DOLLARS

501

$1 \geq 0$ sf
0

$\begin{array}{cc}0 & 78\end{array}$

$0 \quad 012 \quad 50$

0

755

5,788

o

0

5,033

755

5,788

321200.13 SPECIAL CONSTRUCTIOH ERECTED, INSUL, LINER, SKYLIGHT LOUVER, VENT, $3 / 0$ DOOR AND A $12 \times 10$ OVERHEAD DOOR

321200.1345603 HEATED PIPE CHASE BETWEEN NEW ANNEX BUILDING AND EXIST 105KE BASIN BLDG CNO DETAIL AVAIBLE, ESTM ALLOWANCE

321200,1345604 TIE INTÓ EXISTING METAL BUILDING

321200.1345606 ANNEX AIR LOCK

SUBTOTAL SPECIAL CONSTRUCIION

TOTAL COST CODE 50113

HBS 321200

(ESCALATION $1.71 \%$ - CONTINGENCY $30.00 \%)$

1 JOB

0

$1 \mathrm{JOB}$

0

$0 \quad 0$

02000

300

2300

$\begin{array}{llllllllll}501 & 1 & \mathrm{~J} O B & 0 & 0 & 0 & 0 & 5300 & 0 & 795\end{array}$

501

$108 \mathrm{SF}$

$\begin{array}{ccc}0 & 0 & 0 \\ 0 & \ldots & 0\end{array}$

0

$0 \quad 8594$

$0 \quad 1289$

9883

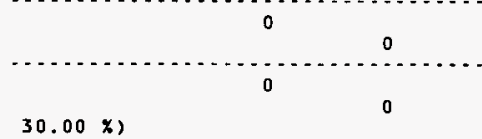

0

53,894

8,084

0

0

53,894

-

8,084

61,978

CONVEYING SYSTEMS

321200.14
321200.1456702 AIR PALLET

SUBTOTAL CONVEYING SYSTEMS

TOTAL COST CODE 50114

HBS 321200

(ESCALATIO

$1.71 \%-$ CONTINGENCY

\begin{tabular}{|c|c|c|c|c|c|c|c|c|c|}
\hline & $1 E A$ & 0 & 0 & 0 & 0 & 22700 & 0 & 3405 & 26105 \\
\hline & & 0 & & 0 & & 22,700 & & 3,405 & \\
\hline & & & 0 & & 0 & & 0 & & 26,105 \\
\hline & & 0 & & 0 & & 22,700 & & 3,405 & \\
\hline 20.00 & x) & & 0 & & 0 & & 0 & & 26. \\
\hline
\end{tabular}

TOTAL HBS 321200 PRE-ENGINEERED METAL BUILDING

536

16,634

o

16,289

83,397

136,249 
WHC-SD-SNF-RPT-012, Rev. 0

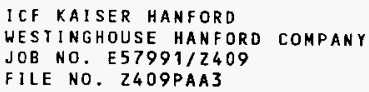

DOE_RO8 - ESTIMATE DETAIL BY HBS / COST CODE
PAGE 82

DATE $07 / 24 / 96 \quad 11: 28: 57$

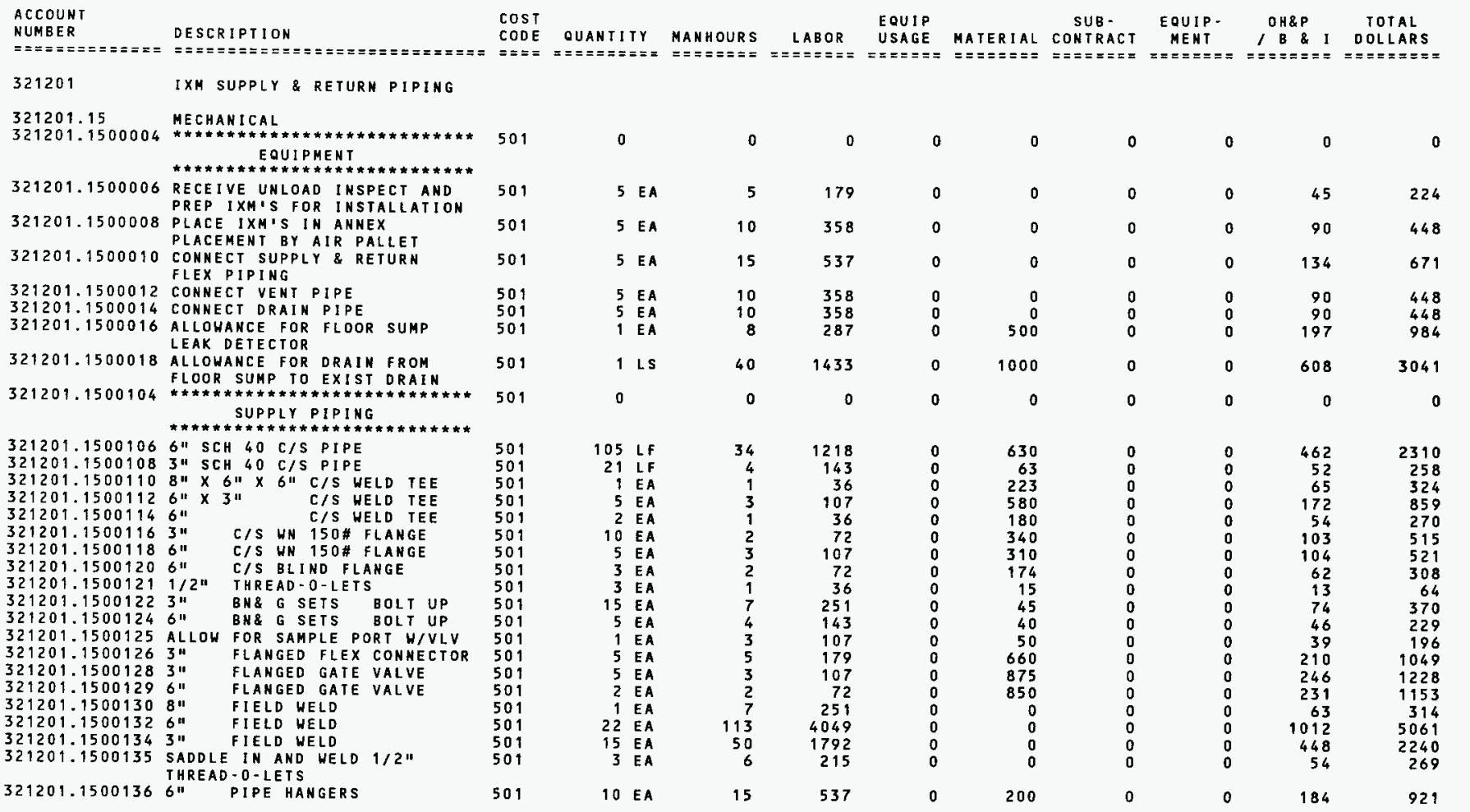


WHC-SD-SNF-RPT-012, Rev. 0

I CF KAISER HANFORD

WESTINGHOUSE HANFORD COMPANY

JOB NO. E57991/Z409

FILE NO. Z409PAA3

ACCOUNT

DESCRIPTION

* IEST - INTERACTIVE ESTIMATING * *

K-BASINS INTEGRATED WATER SYSTEM

PRELIMINARY

DOE_ROB - ESTIMATE DETAIL BY WBS/ COST CODE

NUMBER

QUANTITY MANHOURS

ABOR USAGE
PAGE 83

DATE $07 / 24 / 96 \quad 11: 28: 57$

BY JPM SMF DKH LGH
$======== \pm===$

$321201.15001383 "$ " PIPE HANGERS

321201.1500139 DYE - PEN WELDS

321201.1500140 TEST PIPE

321201.1500942 PAINT PIPE, VALVES \& HGRS ** 501 REIURN PIPING

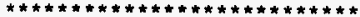

321201.1500206 6" SCH $40 \mathrm{C} / 5 \mathrm{PIPE}$

$321201.15002074 "$ SCH 40 C/S PIPE

321201.150020831 SCH 40 C/S PIPE

$321201.15002092 "$ SCH 40 C/S PIPE

$321201.15002106 \times 4$ C/S CONC. REDUCER

$321201.15002116 \times 3$ C/S CONC. REDUCER

$321201.15002124 \times 2 \quad \mathrm{C} / \mathrm{S}$ CONC. REDUCER

321201.1500213411

$321209.15002146 " \times 3 " \quad$ C/S HELD TEE

$321201.150021661 \times 3 "$ C/S HELD TEE

321201.1500217 .

321201.150021724

321201.150021831

321201.1500219411

$321201.15002206 "$

321201.150022261

321201.150022421

321201.150022631

$321201.1500228 \quad 411$

321201.150023061

321201.1500232 ALLOW
$321201.15002331 / 2 "$

C/S HN 150 H FLAME

C/S HN 150 \# FLANGE

C/S WN 150\# FLANGE

C/S WN 150 FLANGE

C/S BLIND FLANGE

BN\& G SEIS BOLT UP

BN\& G SETS BOLT UP

BN\& G SETS BOLT UP

BN\& G SETS BOLI UP

FOR SANPLE PORT HIVLV

THREAD - 0-LETS

$321201.15002342^{11}$

$321201.15002363 "$

321201.1500238311

$321201.15002394 "$

321201. $15002394 "$

$321201.15002406 "$

$321201.15002426 " 1$

$321201.15002444 "$
321201.150024631

321201.15002482 "

FLANGED FLEX CONNECTOR

FLANGED GATE VALVE

FLANGED GATE VALVE

FLANGED GATE VALVE

FLANDED GATE VALVE

FIELD HELDS

FIELD WELDS

FIELD WELDS

FIELD HELDS

321201.1500250 SADDLE IH AND HELO 1/2"

THREAD - 0 - LET

321201.1500252 SADDLE IN AND HELD $2 "$

THREAD-O-LETS

50

501
501

501

501

501

501

501

501

501

501

501

501

501

501

501

501

501
501

501

501

501

501

501

501

501

501

501

501

501

501
501
501
501
501

SUB-
MATERIAL CONTRACT

EQUIP.

OH\&P

TOTAL

MENT, B \& I DOLLARS

$$
\begin{array}{rl}
5 & E A \\
41 & \text { EA } \\
1 & \text { LS } \\
1 & \text { LS }
\end{array}
$$

105 LF

$10 \leq F$

21 LF

$10 \mathrm{LF}$

1 EA

I EA

2 EA

1 EA

2 EA

$10 \mathrm{EA}$

2 EA

3 EA

3 EA

2 EA

15 EA

2 EA

$3 E A$

6 EA

$6 E A$

5 EA

5 EA

$1 E A$

5 EA

1 EA

1 EA
20 EA

20 EA

8 EA

$2 \mathrm{EA}$

6 EA

5 EA

$\begin{array}{rl}215 & 0 \\ 1935 & 0 \\ 107 & 0 \\ 0 & 0 \\ 0 & 0\end{array}$

75
0
0
0
0

1218

72
143

36

36

36
0

36

107

36
0

72
0

72
72

72
36

251

36
72

645

72
107

179

36

107

36
36

3905

1182

1792

537

$C-9)$

63

60

20

41

41
22

100

580

90
60

340

80

174

6
45

10
24

300

30
75

75
660

660
150

875

225
425

0

o

0

0

0
0
0
400
0

48

2419
134

440

2310

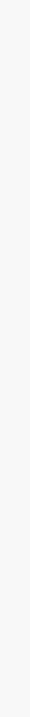

0

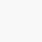

462
33
52
14
19
19
6
34
172
32
15
103
20
65
62
11
74
12
24
236
26
46
210
47
246
65
115
976
296
448
45
108
134

165

258
70

96

96
28

170

859

158
75

515

100

323

53

370

120

1181

1181
128

228

1049

233
1228

1228

326

1478

2240

224

538

671 
WHC-SD-SNF-RPT-012, Rev. 0

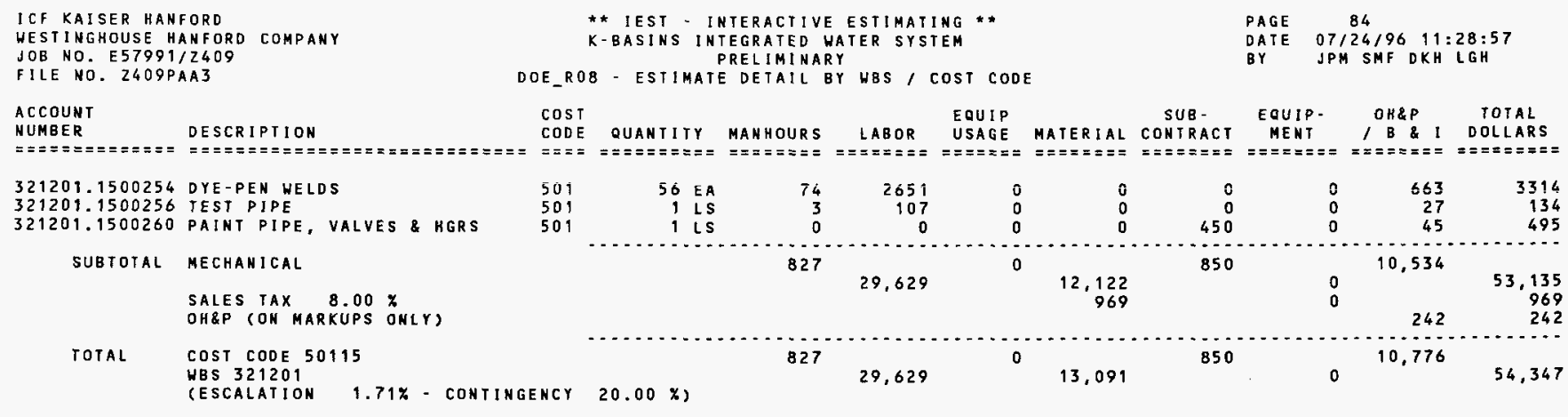

TOTAL WBS 321201 IXM SUPPLY \& RETURN PIPING

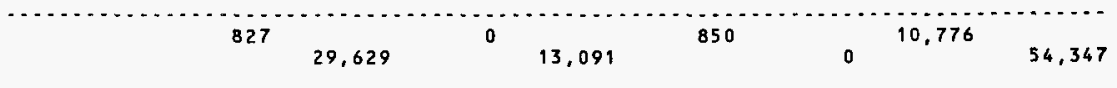


WHC-SD-SNF-RPT-012, Rev. 0

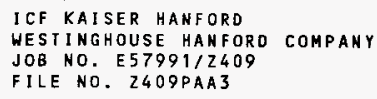

FILE NO. 2409PAA3

PAGE 85

DATE $\quad 07 / 24 / 96 \quad 11: 28: 57$

BY JPM SMF DKH LGH

\section{$\cos T$}

CODE

DE-SCR I PT I OH

QUANTITY MANHOURS LQUIP

SUB -

EQUIP- OH\&P TOTAL NUMBER

IXM DRAIN \& VENT

321202

MECHANICAL

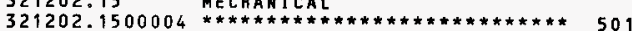
DRAIN \& VENT PIPING

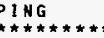

$321202.15000062 "$ SCH 40 C/S PIPE

321202.1500008 2" C/S B-WELD ELLS

$321202.15000102 "$ C/S B-WELD TEES

$321202.15000122 "$ C/S THREADED NIPPLES

321202.1500014211 C/S THREADED UHIONS

321202.1500016 2" BRASS THREADED GATE VALVE

321202.1500018 ALLOH FOR 2" RELIEF VALVE

$321202.15000202 "$ " FIELD WELD

321202.1500022 TEST

321202.1500024 DYE-PEN WELDS

321202.1500026 PAINT PIPE

321202.1500028 ALLOW FOR HEATING OF PIPE

CHASE BETWEEN K-BASIN AND

ANHEX (ELEC HEATER)

SUBTOTAL

\section{MECHANICAL}

SALES TAX $8.00 \%$

OH\&P (ON MARKUPS ONLY)

TOTAL COST CODE 50115

WBS 321202

CESCALATION

$1.71 \%-$ CONTINGENCY

$\begin{aligned} 0 & \\ & \\ 336 & \text { LF } \\ 106 & \text { EA } \\ 8 & \text { EA } \\ 20 & \text { EA } \\ 10 & \text { EA } \\ 5 & \text { EA } \\ 5 & \text { EA } \\ 238 & \text { EA } \\ 1 & \text { JOB } \\ 238 & \text { EA } \\ 2 & \text { JOB } \\ 1 & \text { LS }\end{aligned}$

$\begin{array}{rr}0 & 0 \\ & \\ 37 & 1326 \\ 10 & 358 \\ 1 & 36 \\ 2 & 72 \\ 1 & 36 \\ 2 & 72 \\ 2 & 72 \\ 600 & 21498 \\ 14 & 502 \\ 314 & 11251 \\ 0 & 0 \\ 0 & 0\end{array}$

\section{0}

0

0

o

0 DOLLARS

$\begin{array}{lr}501 & 336 \text { LF } \\ 501 & 106 \text { EA } \\ 501 & 8 \text { EA } \\ 501 & 20 \text { EA } \\ 501 & 10 \text { EA } \\ 501 & 5 \text { EA } \\ 501 & 5 \text { EA } \\ 501 & 238 \text { EA } \\ 501 & 1 \text { JOB } \\ 501 & 238 \text { EA } \\ 501 & 2 \text { JOB } \\ 501 & 1 \text { LS }\end{array}$

672
1060
208
60
90
175
750
0
0
0
0
0

0
0
0
0
0
0
0
0
0
0
1600
600

0
0
0
0
0
0
0
0
0
0
0
0

500
355
61
33
32
62
206
5375
126
2813
160
60

2498

1773

165

158

309

1028

1028

628

14064

1760

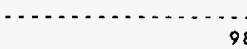

983

2,200

9.783

660

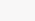

3

3, 015

o $60 \quad \begin{array}{rr}241 \\ 60\end{array}$

983 04100

983

$20.00 \%$

9,843

50,522

35,223

3,256

TOIAL WBS 321202 IXM DRAIN \& VENT

983

9,843

50,522 
WHC-SD-SNF-RPT-012, Rev. 0

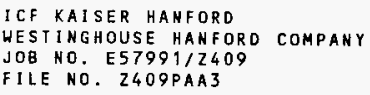

\section{ACCOUNT}

NUMBER DESCRIPIION

COST EQUIP

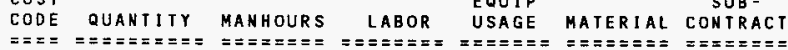

PAGE

DATE $07 / 24 / 96 \quad 11: 28: 57$

BY JPM SMF DKH LGH

\section{COMP/AIR AND SAFEIY SHOWER}

321204.15 MECHANICAL

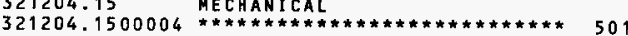
PIPE \& EQUIPMENT

321204.1500006 I IR \#T3030120H 30 HP, 100 ACEM, AIR COMPRESSOR' $H / 120$

321204.1500008 SAFETY SHOHER H/EYE HASH STA 501 321204.1500010 ALLOWANCE FOR C/S COMPRESSED 50 AIR PIPE VALVES \& FITTINGS

321204.1500012 ALLOHANCE FOR C/S SAFETY SHR PIPE VALVES \& FITTINGS

321204.1500014 ALLOHAMCE FOR AIR HOSE INCLUDES HOSE TO PIPE CONNECTIONS

321204.1500016 ALLOWANCE FOR C/S AIR PIPING SUBTOTAL MECHANICAL

SALES TAX $8.00 \%$ OH\&P (ON MARKUPS ONLY)

TOTAL COST CODE 50115 WBS 321204 (ESCALATION $1.71 \%$ - CONTINGENCY $20.00 \%$

$501 \quad 1$

501

$20.00 \%$

TOTAL HBS 321204 COMP/AIR AND SAFETY SHOWER
322
1 EA

1 EA

1 LS

1 LS

1 LS

LS
0

40

1433

430
2866

430
2866

2866

358 $====0===0=0=0=0=0$

EQUIP- OHEP, TOTAL

100

$322 \quad 11,536$

$0 \quad 1000$

789

0

0

0

0

200

500

500

300

1000

0

...

0

0

$0 \quad 1146$

0

9.289
743

$n+\cdots \cdots \cdots$
0

322

11,536

0

10,032

0

$\begin{array}{lr}0 & \\ 0 & 185 \\ 0 & 5,394\end{array}$

209

ร

5729


WHC-SD-SNF-RPT-012, Rev, 0

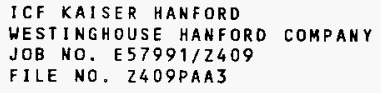

ACCOUNT

DESCR IPIION

HVAC \& HEPA FILTRATION

321205 MECHANICA

321205.15

501 DOE_R08

ESIIMATE DETAIL BY HBS / COST CODE

CODE QUANTITY MANHOURS LABOR USAGE MATERIAL CONTRAC

PAGE

DATE $07 / 24 / 96 \quad 11: 28: 57$

BY JPM SMF DKH LGH
321205.1500016 ALLOWANCE FOR HEAPA FILTER
2 STAGE HEPA FILTER 1 IX1
321205.1500016 ALLOWANCE FOR HEAPA FILTER
2 STAGE HEPA FILTER IX1
321205.1500016 ALLOWANCE FOR HEAPA FILTER
2 STAGE HEPA FILTER 1 IX1
HITEST SECTION \& TRAIH

SUBTOTAL MECHANICAL

TOTAL

\section{COST CODE 50115
HBS 321205}

(ESCALATIOH

$1.71 \%$ - CONTINGENCY

TOTAL HBS 321205 HVAC \& HEPA FILTRAIION
SUB

NTRACT

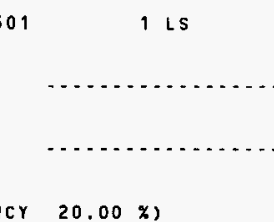

$$
0
$$

$0_{0}$

$\ldots . .$.
0
0

0

EQUIP -

MENT

OH\&P

, $\mathrm{B} \& 1$

TOTAL

OLLARS

(2)

0

20,000

.

o

0

0

20000

0

2000

22000

2,000 22,000

22,000

$\begin{array}{ll}0,000 & 22,000\end{array}$

0

0
0

0

20,000

2,000 
WHC-SD-SNF-RPT-012, Rev. 0

ICF KAISER HANFORD

WESTINGHOUSE HANFORD COMPANY

JOB NO. ES7991/2409

FILE NO. Z409PAA3

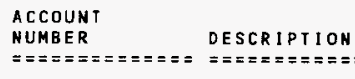

330000

BURIAL FEES

330000.15

15 MECHANICAL

5 EACH BURIAL DRUMS AT 7.35700 CF EACH ASSUME LLW DRUM USE LOCATIONS DEBRIS REMOVAL PIPE TIE IN

330000.1500006 WEASEL PIT PUMP TIE IN INSTRUMENTATIOH TIE IN

CHILLER BAY PIPE IIE IN

TRANSFER BAY PIPE TIE IN

330000.150001012 EACH BURAAL BOXES AT 128

CF EACH ASSUME LLW

BOX USE LOCATIONS

WEASEL PIT GRATIHG 1 BOX

330000.1500012 GRH/HSE STG/SLAB

$\begin{array}{ll}2 & \text { BOX } \\ 6 & \text { BOX }\end{array}$

700

* * IEST - INTERACTIVE ESTIMATING *

K-BASINS INTEGRATED HATER SYSTEM$$
\text { PRELIMINARY }
$$

DOE RO8 - ESTIMATE DETAIL BY HES / COST CODE

$\cos$

COST
CODE QUANTITY MANHOURS LABOR UQUIP
USAGE

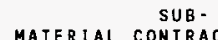

PAGE 88

DATE $07 / 24 / 96 \quad 11: 28: 57$

BY

JPM SMF DKH LGH

700

$37 \mathrm{CF}$

0

0

0

o

2220

EQUIP -

OH\&P, TOTAL

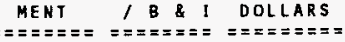

0

0

0

0

0

o

0

$0 \quad 92160$

0

0

$1536 C$

0

0

0

0

0

0

0

SUBTOTAL MECHANICAL

TOTAL

COST CODE 70015

H8S 330000

(ESCALATION

$1.71 \%$ - CONT INGENCY

92160

0

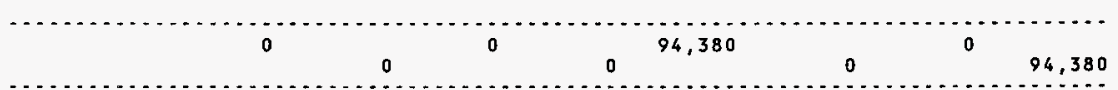

$30.00 \%)$

0

0

94,380

o

0

94,380

TOTAL WBS 330000 BURIAL FEES

0

$\mathbf{0}$

0

94,380

0

94,380 
WHC-SD-SNF-RPT-012, Rev. 0

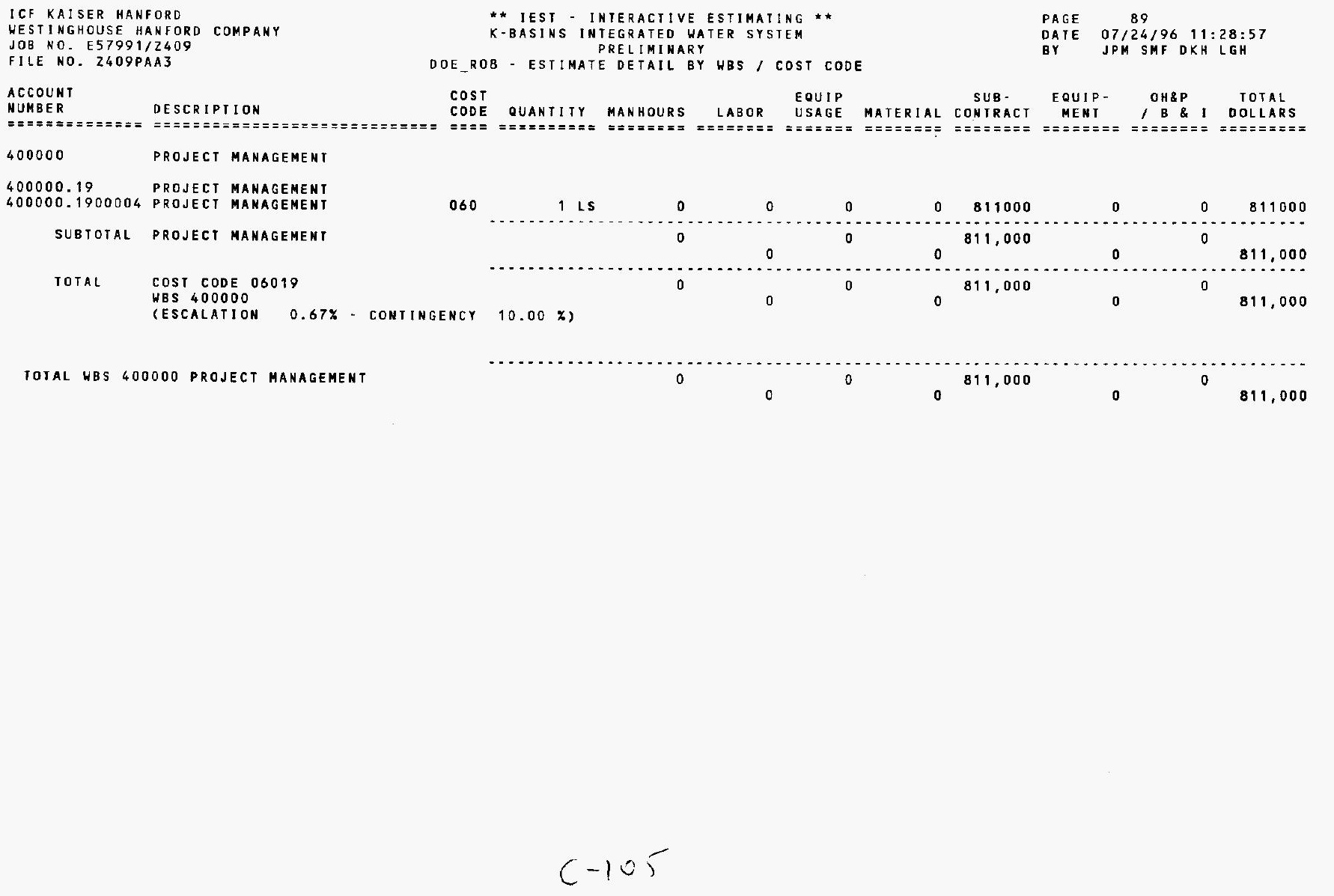


WHC-SD-SNF-RPT-012, Rev. 0

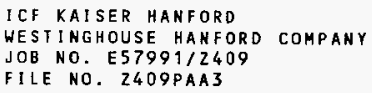

\begin{tabular}{|c|c|c|c|c|c|c|c|c|c|c|}
\hline $\begin{array}{l}900 \\
900\end{array}$ & $\begin{array}{l}1 \\
1\end{array}$ & $\begin{array}{l}\text { LS } \\
\text { LS }\end{array}$ & $\begin{array}{l}0 \\
0\end{array}$ & $\begin{array}{l}0 \\
0\end{array}$ & $\begin{array}{l}0 \\
0\end{array}$ & $\begin{array}{l}0 \\
0\end{array}$ & $\begin{array}{l}73000 \\
12434\end{array}$ & $\begin{array}{l}0 \\
0\end{array}$ & $\begin{array}{l}0 \\
0\end{array}$ & $\begin{array}{l}73000 \\
12434\end{array}$ \\
\hline 900 & 1 & LS & 0 & 0 & 0 & 0 & 12434 & 0 & 0 & 12434 \\
\hline 900 & 1 & LS & 0 & 0 & 0 & 0 & 12434 & 0 & 0 & 12434 \\
\hline 900 & 1 & LS & 0 & 0 & 0 & 0 & 12434 & 0 & 0 & 12434 \\
\hline 900 & 1 & LS & 0 & o & 0 & 0 & 24868 & 0 & 0 & 24868 \\
\hline 900 & 1 & Ls & 0 & 0 & 0 & 0 & 6217 & 0 & 0 & 6217 \\
\hline 900 & 1 & LS & 0 & 0 & 0 & 0 & 6217 & 0 & 0 & 6217 \\
\hline 900 & 1 & LS & 0 & 0 & 0 & 0 & 6217 & 0 & 0 & 6217 \\
\hline 900 & 1 & LS & 0 & 0 & 0 & 0 & 12434 & 0 & 0 & 12434 \\
\hline 900 & 1 & LS & 0 & 0 & 0 & 0 & 24868 & 0 & 0 & 24868 \\
\hline 900 & 1 & LS & 0 & 0 & 0 & 0 & 12434 & 0 & 0 & 12434 \\
\hline
\end{tabular}


WHC-SD-SNF-RPT-012, Rev. 0

ICF KAISER HANFORD

WESTINGHOUSE HANFORD COMPANY

JOB NO. E57991/2409

FILE NO. 2409PAA3

ACCOUN T

NUMBER DESCRIPTION

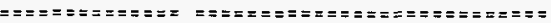

500096.9000056 FY96 BACKWASH FILIER DEMO (ENG INEERING SUPPORT)

500096.9000060 FY96 CONSULTANTS,

VULNERABILITY

(ENGINEERING SUPPORT)

IOTAL WBS 500096 FY 96 OTHER PROJECT COST
* * IEST - INTERACTIVE ESTIMATING **

K-BASINS INTEGRATED HATER SYSTEM

$$
\text { PRELIMINARY }
$$

DOE_ROB - ESTIMATE DETAIL BY WBS, COST CODE

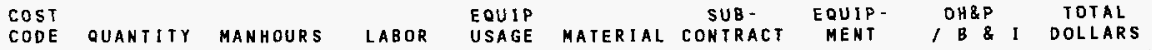

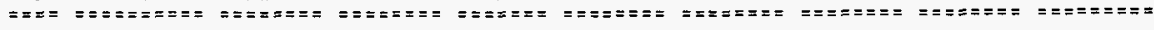

\begin{tabular}{|c|c|c|c|c|c|c|c|c|c|}
\hline 900 & 1 Is & 0 & 0 & 0 & 0 & 260000 & 0 & 0 & 260000 \\
\hline 900 & $1 \mathrm{LS}$ & 0 & 0 & 0 & 0 & 52000 & 0 & 0 & 52000 \\
\hline
\end{tabular}




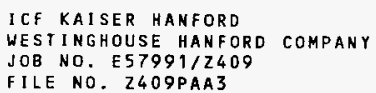

500097.9000052 FY97 REVIEH ALARA ASPECTS (ENGINEERING SUPPORT)
* * IEST - INTERACTIVE ESTIMATING * *

K-BASINS INTEGRATED WATER SYSTEM

$$
\text { PRELIMINARY }
$$

DOE R08 - ESTIMATE DETAIL BY WBS / COST CODE

PAGE
DATE
BY
BY $/ 24 / 96 \quad 11: 28: 58$

BY JPM SMF DKH LGH

\begin{tabular}{|c|c|c|c|c|c|c|c|c|c|c|c|}
\hline $\begin{array}{l}\operatorname{COS} 1 \\
\operatorname{CODE} \\
=== \pm\end{array}$ & $\begin{array}{l}\text { QUANTIT } \\
=\approx======\end{array}$ & $\begin{array}{l}\text { Tr } \\
===\end{array}$ & $\begin{array}{l}\text { MANHOURS } \\
========\end{array}$ & $\begin{array}{c}\text { lABOR } \\
======\end{array}$ & $\begin{array}{l}\text { EQUIP } \\
\text { USAGE } \\
== \pm===\end{array}$ & $\begin{array}{l}\text { MATERIAL } \\
======\end{array}$ & $\begin{array}{c}\text { SUB - } \\
\text { CONTRACT } \\
=======\end{array}$ & $\begin{array}{c}\text { EQUIP - } \\
\text { MENT } \\
=======\end{array}$ & 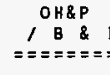 & $=$ & $\begin{array}{c}\text { TOTAL } \\
\text { OOLLARS } \\
=========\end{array}$ \\
\hline 900 & 1 & LS & 0 & 0 & 0 & 0 & 392000 & 0 & & 0 & 392000 \\
\hline 900 & 1 & LS & 0 & 0 & 0 & 0 & 62000 & 0 & & 0 & 62000 \\
\hline 900 & 1 & LS & 0 & 0 & 0 & 0 & 124000 & 0 & & 0 & 124000 \\
\hline 900 & 1 & LS & 0 & 0 & 0 & 0 & 124000 & 0 & & 0 & 124000 \\
\hline 900 & 1 & LS & 0 & 0 & 0 & 0 & 62000 & 0 & & 0 & 62000 \\
\hline 900 & 1 & LS & 0 & 0 & 0 & 0 & 124000 & 0 & & 0 & 124000 \\
\hline 900 & 1 & LS & 0 & 0 & 0 & 0 & 31000 & 0 & & 0 & 31000 \\
\hline 900 & 1 & LS & 0 & 0 & 0 & 0 & 12400 & 0 & & 0 & 12400 \\
\hline 900 & 1 & is & 0 & 0 & 0 & 0 & 12400 & 0 & & 0 & 12400 \\
\hline 900 & 1 & LS & 0 & 0 & 0 & 0 & 62000 & 0 & & 0 & 62000 \\
\hline 900 & 1 & LS & 0 & 0 & 0 & 0 & 49600 & 0 & & 0 & 49600 \\
\hline 900 & 1 & LS & 0 & 0 & 0 & 0 & 49600 & 0 & & 0 & 49600 \\
\hline
\end{tabular}


WHC-SD-SNF - RPT-012, Rev. 0

ICF KAISER HANFORD

WESTINGHOUSE HANFORD COMPANY

JOB NO. E57991/Z409

FILE NO. 2409 PAA3

ACCOUNT

NUMBER

DESCRIPTION

800000 40ups

TOTAL WBS 500097 FY 97 OTHER PROJECT COST
* * IEST - INTERACTIVE ESTIMATING * *

K-BASINS INTEGRATED WATER SYSTEM

PRELIMINARY

DOE_ROB - ESTIMATE DETAIL BY WBS / COST CODE
PAGE 93

DATE $07 / 24 / 96 \quad 11: 28: 58$

BY JPM SMF OKH LGH $\cos T$

CODE QUANTITY MANHOURS

USAGE

SUB -
MATERIAL CONTRACI

EQUIP -

MEN T

$\ldots+\cdots+\cdots$

0

0

o

$0^{1,}$

105,000

0

0

$1,105,000$

$c \cdot 109$ 
WHC-SD-SNF-RPT-012, Rev. 0

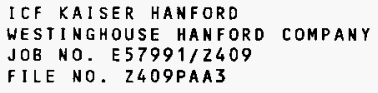

TOTAL W8S 500098 FY 98 OTHER PROJECT COST
* * IEST - INTERACTIVE ESTIMATING * *

K-BASINS INTEGRATED HATER SYSTEM

PRELIMINARY

DOE_ROS - ESTIMATE DETAIL BY HBS / COST CODE

COST EQUIP

CODE QUANTITY MANHOURS LABOR USAGE

SUB -

MATERIAL CONTRACT

PAGE 94

DATE $07 / 24 / 96 \quad 11: 28: 58$

BY JPM SMF DKH LGH

MENT $/$ B \& I DOLLARS

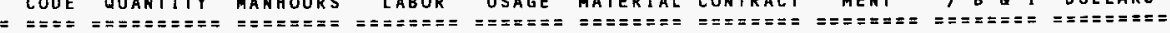

\begin{tabular}{|c|c|c|c|c|c|c|c|c|c|c|}
\hline 900 & 1 & LS & 0 & 0 & 0 & 0 & 310000 & 0 & 0 & 310000 \\
\hline 900 & 1 & LS & 0 & 0 & 0 & 0 & 92970 & 0 & 0 & 92970 \\
\hline 900 & 1 & is & o & 0 & 0 & 0 & 92970 & 0 & 0 & 92970 \\
\hline 900 & 1 & LS & 0 & 0 & 0 & 0 & 92970 & 0 & 0 & 92970 \\
\hline 900 & 1 & LS & 0 & 0 & 0 & 0 & 92970 & 0 & 0 & 92970 \\
\hline 900 & 1 & Ls & 0 & 0 & 0 & 0 & 92970 & 0 & D & 92970 \\
\hline 900 & 1 & LS & 0 & 0 & 0 & 0 & 24792 & 0 & 0 & 24792 \\
\hline 900 & 1 & Is & 0 & 0 & 0 & 0 & 24792 & 0 & 0 & 24792 \\
\hline 900 & 1 & LS & 0 & 0 & 0 & 0 & 24792 & 0 & 0 & 24792 \\
\hline 900 & 1 & Ls & 0 & 0 & 0 & 0 & 24792 & 0 & 0 & 24792 \\
\hline
\end{tabular}




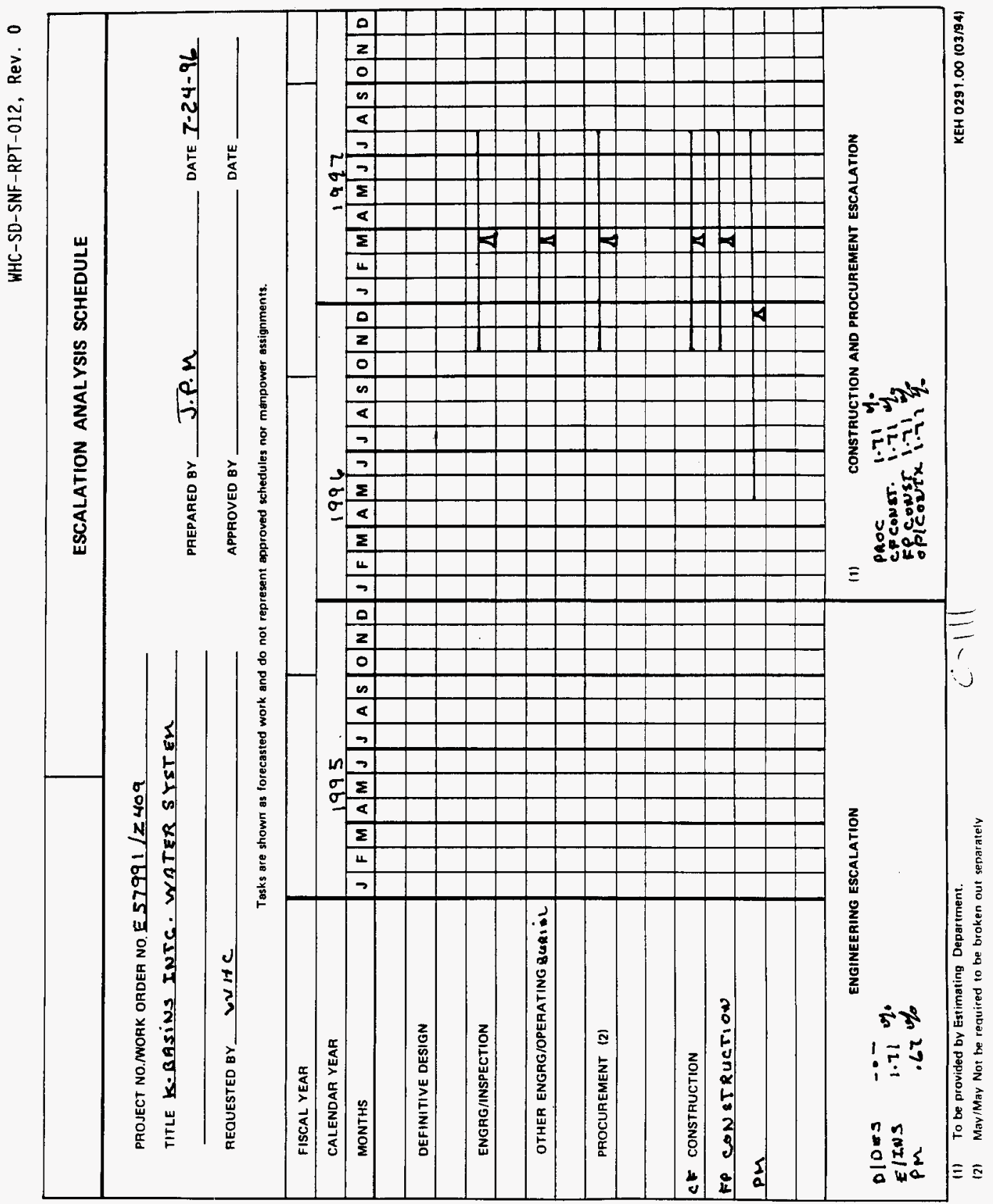


SPENT NUCLEAR FUEL PROJECT

\section{INTEGRATED WATER TREATMENT SYSTEM SCHEDULE}

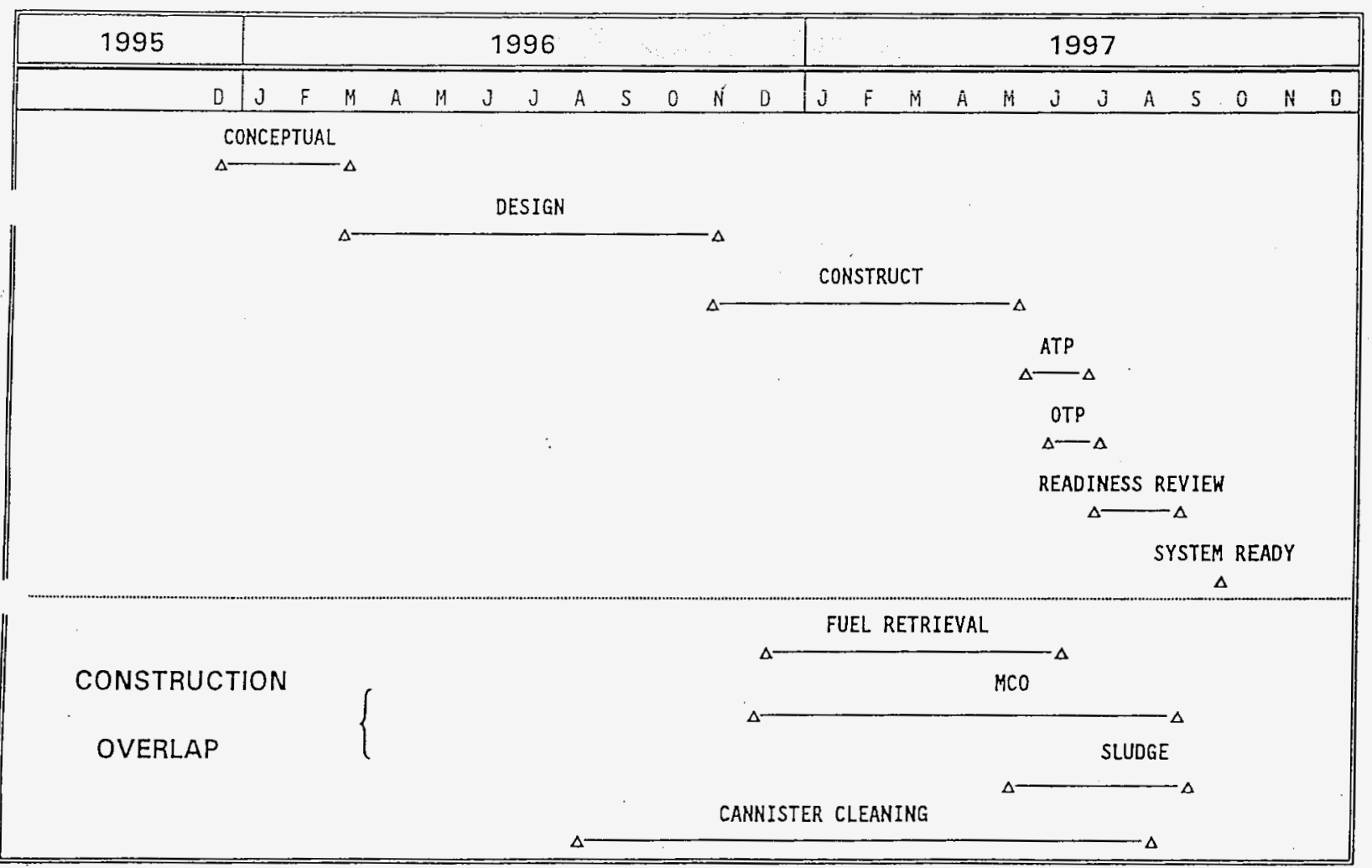

FIGURE 1

(RECOMMENDATION 3. ACQUISITION STRATEGY)

$c-112$ 


\section{COMPOSITE LABOR \& MATERIALS}

\section{USE FOR JUL-96}

\begin{tabular}{|c|c|c|c|c|c|c|c|c|c|c|c|c|}
\hline YEAR & SAN & $\mathrm{FEB}$ & MAR & APR & MAY & S X & (x) & $\mathrm{AUG}$ & YYSEP & 001 & $y_{4} \mathrm{NOV}$ & X \\
\hline 1996 & & & & & & & & 0.23 & 0.46 & 0.67 & 0.88 & 1.08 \\
\hline 1997 & 1.29 & 1.50 & 1.71 & 1.92 & 2.13 & 2.34 & 2.55 & 2.76 & 2.97 & 3.19 & 3.40 & 3.61 \\
\hline 1998 & 3.82 & 4.04 & 4.25 & 4.47 & 4.68 & 4.90 & 5.11 & 5.33 & 5.55 & 5.78 & 6.02 & 6.25 \\
\hline 1999 & 6.49 & 6.73 & 6.96 & 7.20 & 7.44 & 7.68 & 7.92 & 8.16 & 8.40 & 8.66 & 8.92 & 9.17 \\
\hline 2000 & 9.44 & 9.70 & 9.96 & 10.22 & 10.48 & 10.75 & 11.01 & 11.28 & 11.54 & 11.82 & 12.09 & 12.37 \\
\hline 2001 & 12.65 & 12.92 & 13.20 & 13.48 & 13.76 & 14.04 & 14.32 & 14.60 & 14.89 & 15.17 & 15.45 & 15.74 \\
\hline 2002 & 16.02 & 16.31 & 16.60 & 16.89 & 17.17 & 17.46 & 17.75 & 18.04 & 18.33 & 18.63 & 18.92 & 19.21 \\
\hline 2003 & 19.51 & 19.80 & 20.10 & 20.39 & 20.69 & 20.99 & 21.28 & 21.58 & 21.88 & 22.18 & 22.49 & 22.79 \\
\hline
\end{tabular}




\section{OPC ESCALATION RATES}

USE FOR JUN-96

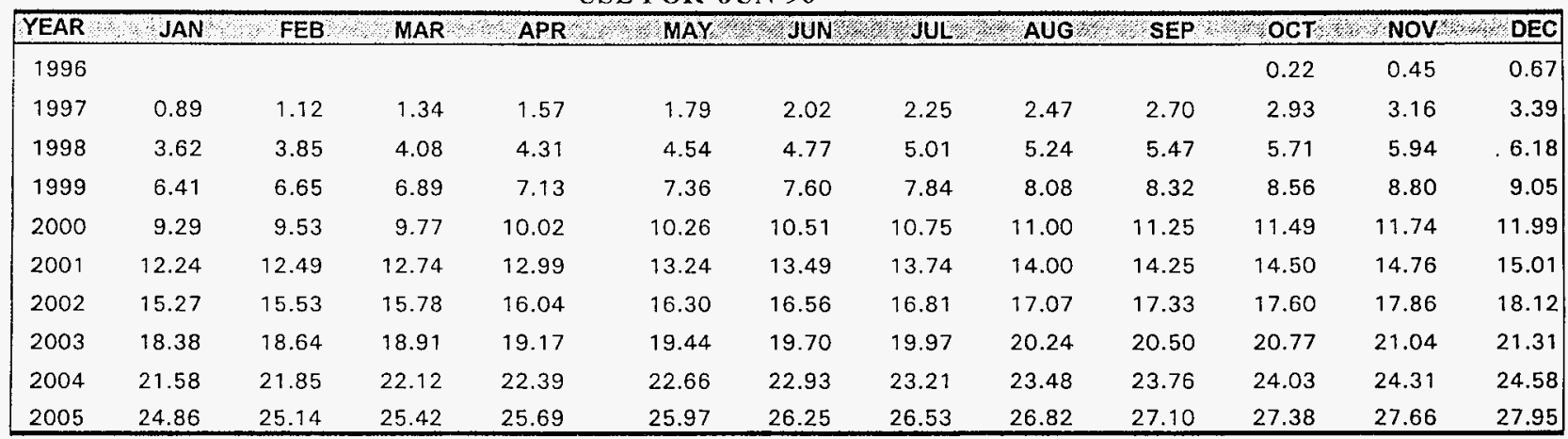


WHC-SD-SNF-RPT-012, Rev. 0

ICF KAISER HANFORD

WESTINGHOUSE HANFORD COMPANY

JOB NO. E57991/Z409PAA1

* * IEST - INTERACTIVE ESTIMATING *

K-BASINS INTEGRATED WATER SYSTEM

PRELI IINARY

VENDOR INFORMATION SHEET
PAGE OF

DATE $07 / 02 / 96 \quad 11: 38: 20$

BY JPM SMF DKH LGH

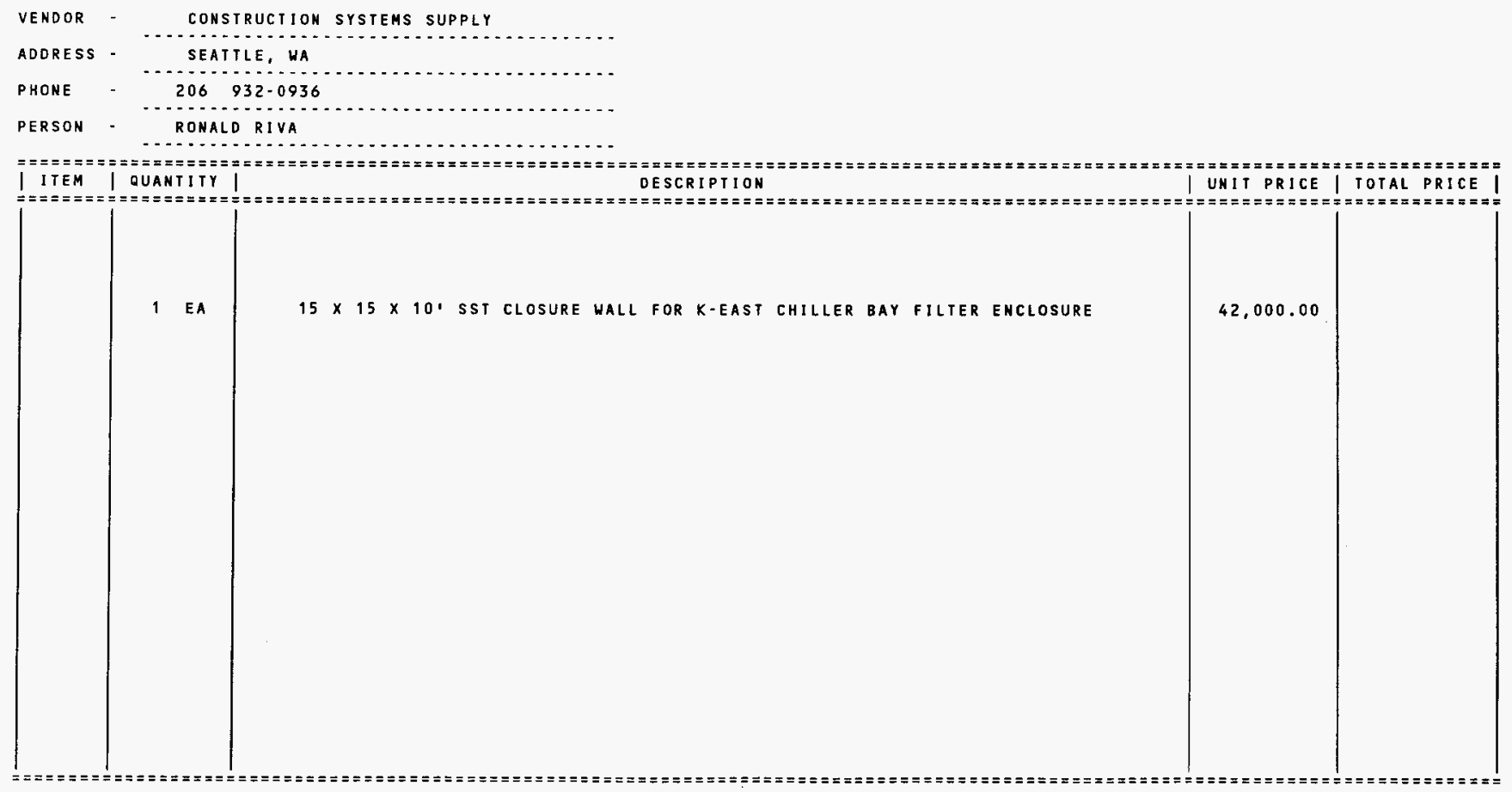


WHC-SD-SNF-RPT-012, Rev. 0

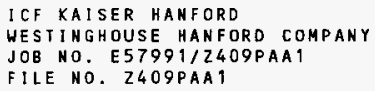

* * IEST - INTERACTIVE ESTIMATING * * K-BASINS INTEGRATED HATER SYSTEM PRELIMINARY
PAGE 0 OF $11: 38: 11$

BY

VENDOR INFORMATION SHEET

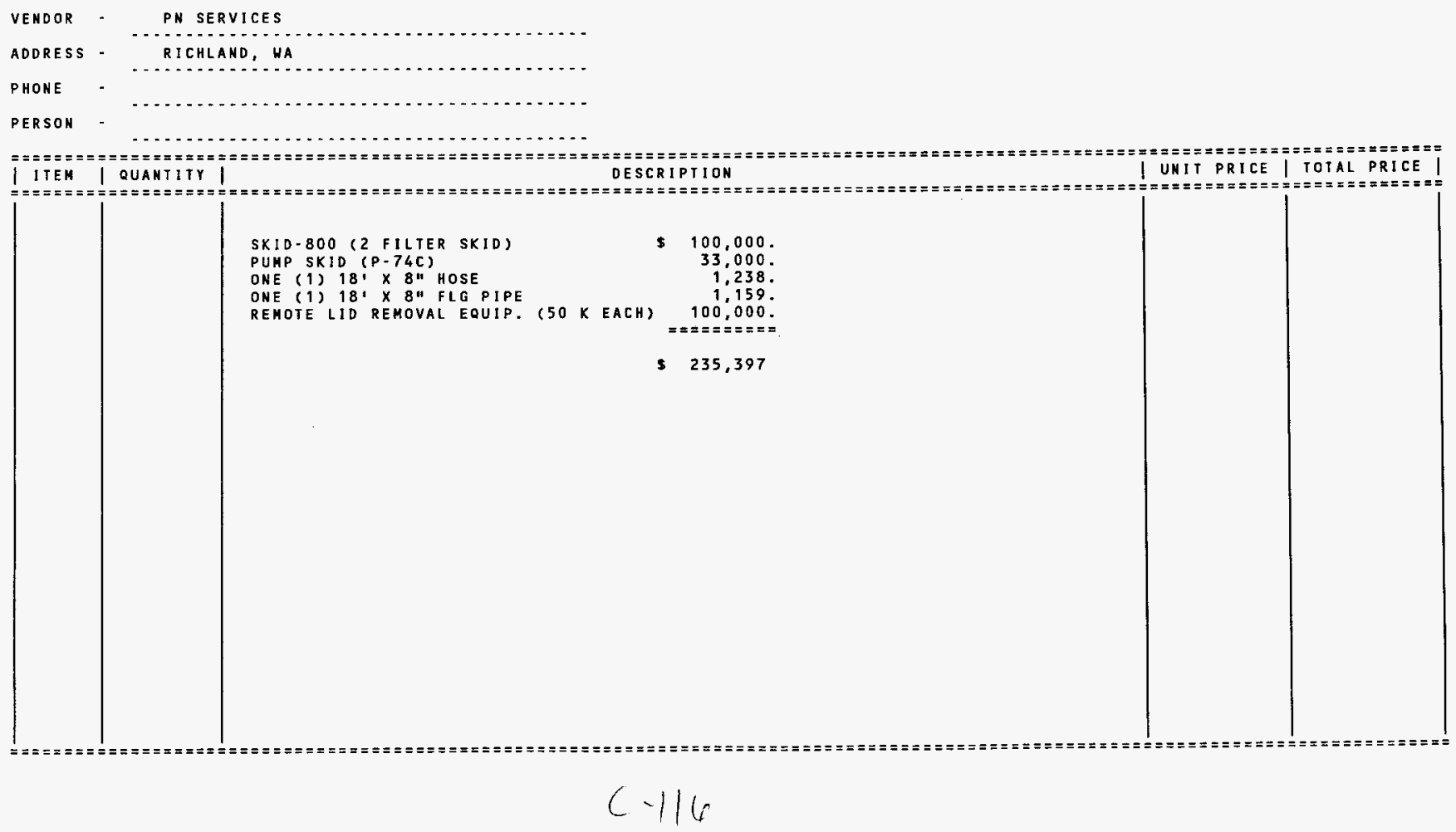


I.HC-SD-SNF-RPT-012, Rev. 0

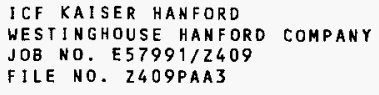

REPORT TOTAL
* * IEST - INTERACTIVE ESTIMATING **

K-BASINS INTEGRATED HATER SYSTEM

$$
\text { PRELI IMINARY }
$$

DOE_ROB - ESTIMATE DETAIL BY WBS / COST CODE

COST
CODE QUANTITY MANHOURS LABOR EQUIP

IPE MATERIAL CONTRACT

$\begin{array}{lc}\text { PAGE } & 95 \\ \text { DATE } & 07 / 24 / 96 \quad 11: 28: 58\end{array}$

BY JPM SMF OKH LGH $\begin{array}{lll}\text { OUIP - } & \text { OH\&P } \\ \text { MENT } & \text { B \& I DOLLARS }\end{array}$

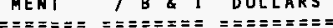

47,742 2,357,000 $6,500 \quad 2,204,580^{5,802,795}$

$0^{1}$

$1,056,662$

$11,427,538$ 
WHC-SD-SNF-RPT-012, Rev. O

\section{APPENDIX D}

\section{PROJECT SCHEDULE}




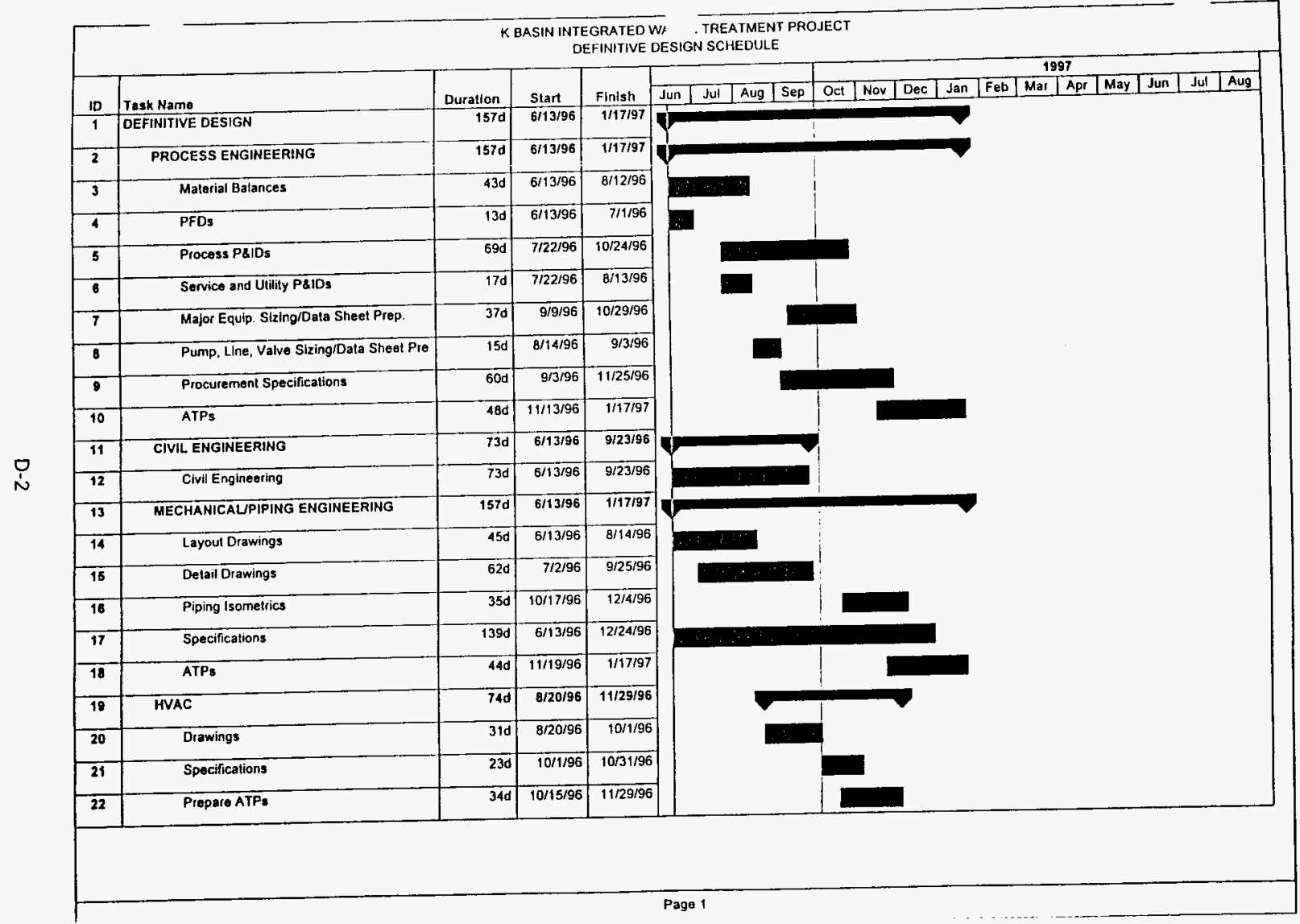




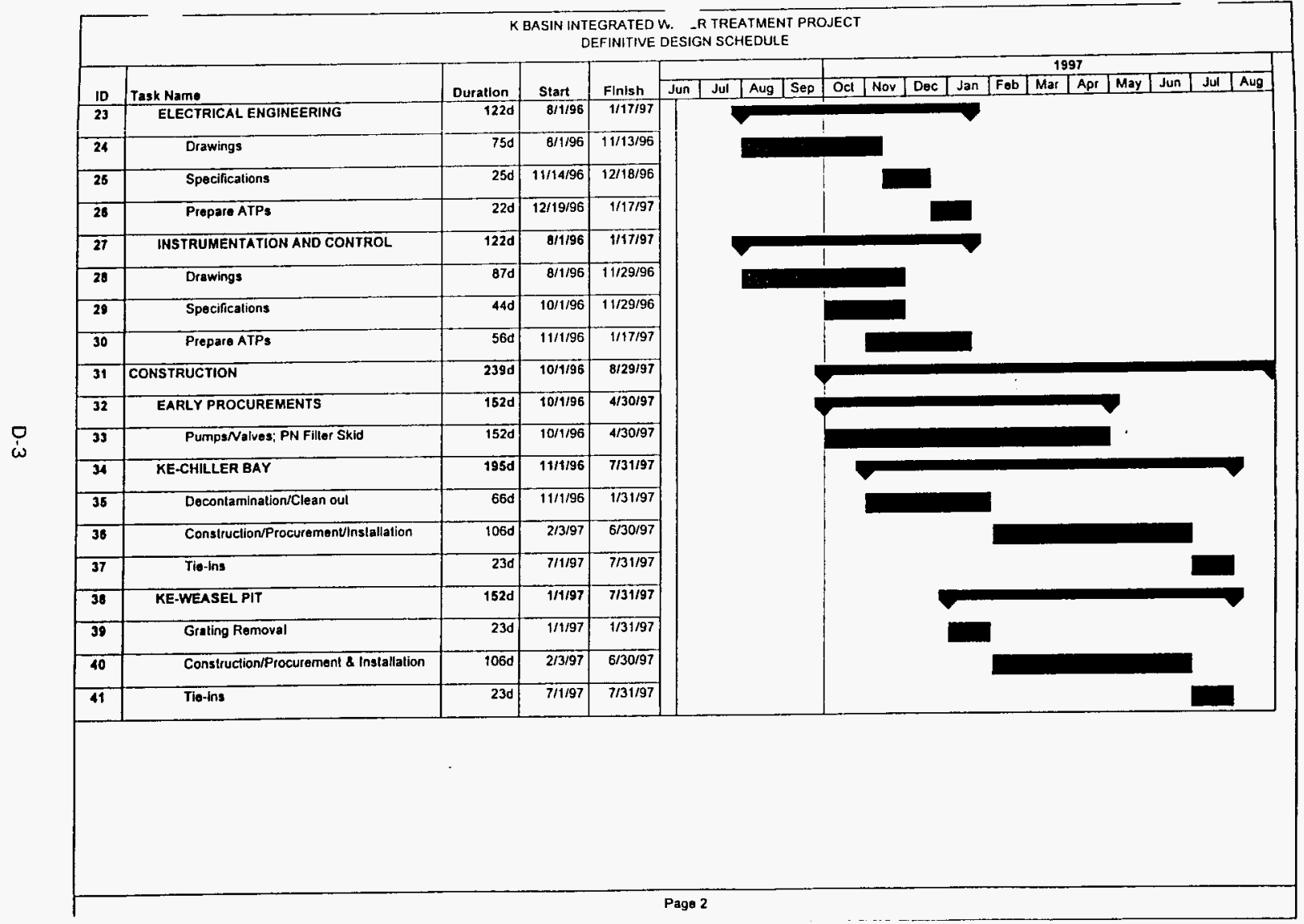




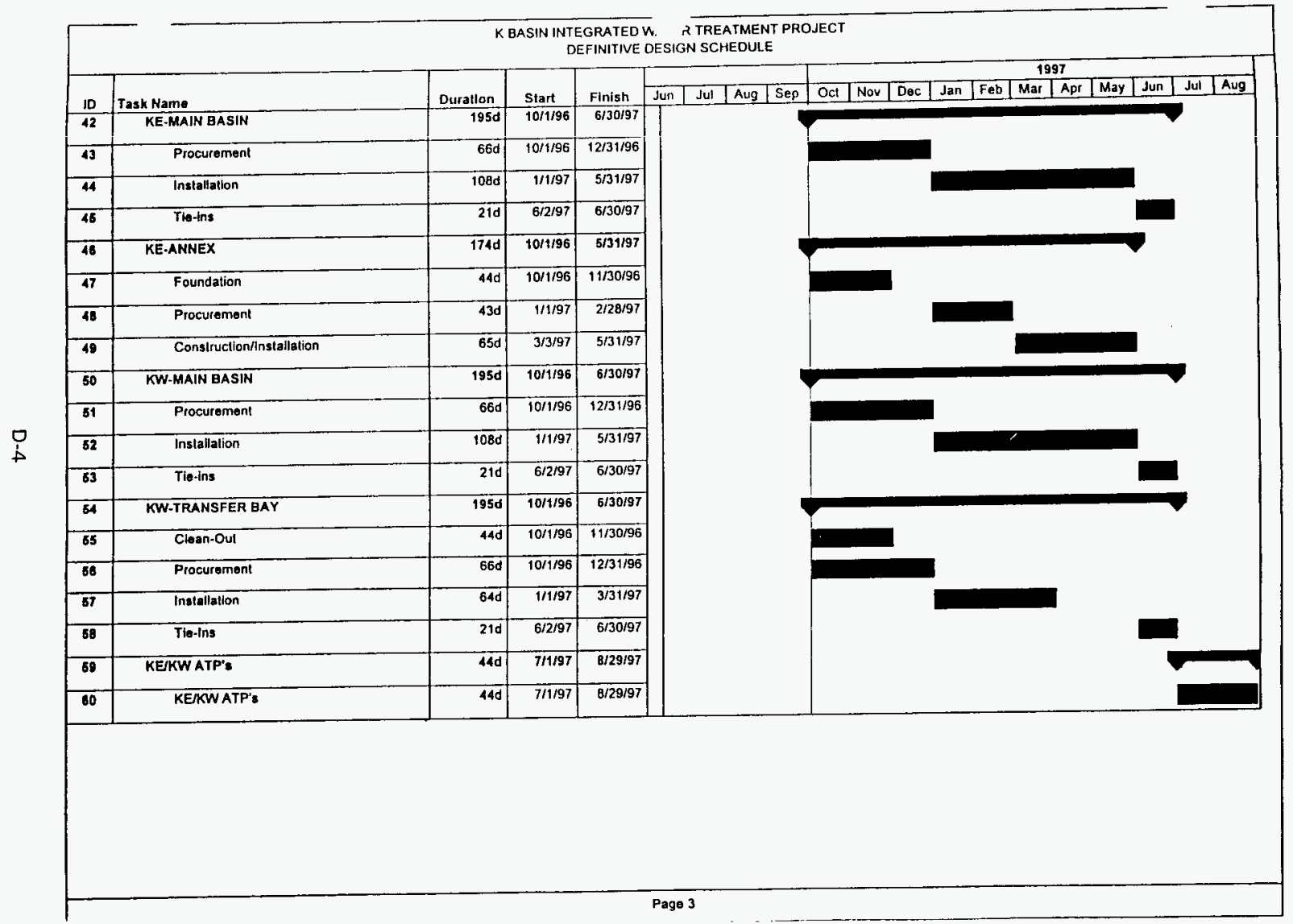




\section{DISTRIBUTION SHEET}

\begin{tabular}{|c|c|c|c|c|c|}
\hline \multirow{2}{*}{$\begin{array}{l}\text { To } \\
\text { Distribution }\end{array}$} & \multirow{2}{*}{\multicolumn{3}{|c|}{$\begin{array}{l}\text { From } \\
K \text { Basins Projects }\end{array}$}} & \multicolumn{2}{|l|}{ Page 1 of 1} \\
\hline & & & & \multicolumn{2}{|c|}{ Date $8-06-96$} \\
\hline \multicolumn{4}{|l|}{ Project Title/Work Order } & \multicolumn{2}{|c|}{ EDT No. 615609} \\
\hline \multicolumn{4}{|c|}{$\begin{array}{l}\text { PROJECT A.9 K EASINS INTEGRATED WATER TREATMENT SYSTEMS } \\
\text { PRELIMINARY DESIGN REPORT }\end{array}$} & \multicolumn{2}{|l|}{ ECN No. } \\
\hline Name & MSIN & $\begin{array}{c}\text { Text } \\
\text { With All } \\
\text { Attach. }\end{array}$ & Text Only & $\begin{array}{l}\text { Attach./ } \\
\text { Appendix } \\
\text { Only }\end{array}$ & $\begin{array}{l}\text { EDT/ECN } \\
\text { Only }\end{array}$ \\
\hline
\end{tabular}

U.S. Department of Energy. Richland Operations Office

J.M. Escami110

$\begin{array}{ll}57-41 & X \\ 57-41 & X\end{array}$

E.D. MacAlister

$X$
$X$

Los Alamos Technical Associates/British Nuclear Fuels

D.E. Ball

Bl-44 X

West inghouse Hanford Company

K.H. Bergsman

S.P. Burke

R3-48 $\quad X$

C. Defigh-Price

D.C. Derosa

$\times 3-74$

$\times 3-79$

$\times 3-85$

D.D. Faris-Zyph

D.0. Hess

V.L. Hoefer

G.S. Hunacek

C.D. Kirk

J.M. Kurta

R.H. Meichle

T.R. Pauly

C.C. Pitkoff

J.P. Schmidt

$\times 3-72$

$\times 3-72$

R3-11

$\times 3-79$

$\times 3-72$

$\times 3-74$

$\times 3-79$

$\times 3-85$

R3-48

$\times 3-78$

D.L. Sherrel1

R3-86

D.W. Siddoway

$\times 3-71$

$\times 3-85$

D.S. Takasumi

$\times 3-85$

$\times 3-85$

$x$
$x$
$x$
$x$
$x$
$x$
$x$
$x$
$x$
$x$
$x$
$x$
$x$
$x$
$x$
$x$
$x$
$x$
$x$

A. 9 Project Files 